\title{
Drivers and Impacts of Atmospheric Rivers in New Zealand
}

by

Daemon Kennett

\author{
A thesis \\ submitted to the Victoria University of Wellington \\ in fulfilment of the \\ requirements for the degree of \\ Master of Science \\ in Geophysics.
}

Victoria University of Wellington 2021 



\begin{abstract}
Atmospheric Rivers (ARs) are long, narrow jets of intense water vapour flux that are a fundamental component of the global atmospheric circulation, transporting moisture and heat from the tropics to higher latitudes. When an AR makes landfall, especially in areas of steep topography, it releases much of its water vapour as precipitation through orographic uplift. Thus, although ARs play a positive role in the distribution and maintenance of water resources in the mid-latitudes, they are also associated with extreme precipitation and flooding. AR events in New Zealand have had major socio-economic consequences with losses to property, farmland, stock, roads and bridges. However, despite knowledge of their occurrence, focused investigations of ARs in New Zealand have received relatively little scientific attention. In particular, little is known about how large-scale climate patterns, such as the Southern Annular Mode (SAM) and El Niño-Southern Oscillation (ENSO), influence ARs and AR-related precipitation extremes.
\end{abstract}

The aim of this study is to quantify the impacts and large-scale drivers of AR landfalls in New Zealand. We employ a new AR detection algorithm, developed specifically for the New Zealand case, to investigate landfalling ARs over a 41-year period from 1979-2019. We investigate the general climatology of ARs, and evaluate the synoptic conditions that drive these events. Using a comprehensive daily rainfall dataset comprising 189 stations, we also investigate the impacts of ARs on NZ rainfall and flooding events. For northern and western regions, over $45 \%$ of rainfall fell directly under AR conditions, contributing to daily rainfall totals 2.5 times higher on average compared to non-AR days. Further, we find that AR days were associated with up to $70 \%$ of daily rainfall totals above the 99th percentile, with insurance damages exceeding NZ \$1.4 billion since 1980.

Finally, for the first time in New Zealand, we investigate how large-scale climate patterns influence the occurrence of ARs. We find that changes in the leading modes of climate variability can alter seasonal and regional AR frequency by upwards of $30 \%$. The SAM is identified as the dominant driver of AR activity (other than the seasonal cycle), with the posi- 
tive SAM phase associated with a $16 \%$ reduction in AR occurrence during summer (30-35\% reduction for the North Island). The links between AR occurrence and ENSO were less clear, though a few statistically significant relationships were found. The Madden-Julian Oscillation (MJO), the leading mode of intraseasonal tropical variability, was found to significantly influence the frequency and timing of AR landfalls (particularly for the northern North Island). Favourable MJO phases were associated with positive AR frequency anomalies $+60 \%$ above the mean. These results demonstrate potential use of the AR framework in skilful subseasonal-toseasonal forecasts of extreme rainfall in New Zealand. 


\section{Acknowledgments}

I would like to thank my primary supervisor Dr Jim McGregor for his guidance, friendly advice, and sharing his wealth of experience. Thank you for giving me the confidence to explore and express my ideas, and encouraging me to pursue a research career.

Many thanks to Dr Kyle Clem for his valuable feedback and encouragement, without which this thesis would not have been possible. I'm extremely grateful for the opportunity to collaborate this past year.

Financial support for this research was provided by the Rachael Westergaard Memorial Scholarship in Geophysics 2020. I extend a special thank you to the Westergaard family for their generosity. Thank you for providing me the opportunity to learn and grow as a scientist.

Finally, a big thank you to my family for their constant support in everything I do. It has been an enjoyable year. 


\section{Contents}

1 Introduction $\quad 1$

1.1 Background ..................... 2

1.1.1 Atmospheric River Definition . . . . . . . . . . . . 2

1.1.2 Atmospheric Circulation . . . . . . . . . . . . 5

1.1.3 Atmospheric River Detection . . . . . . . . . . . . 8

1.1.4 Global and Regional AR Studies . . . . . . . . . . . 10

1.1.5 New Zealand Climate . . . . . . . . . . . . . . . . . 11

1.1.6 New Zealand AR Studies . . . . . . . . . . . . . . . 13

1.1.7 Emerging Fields in AR Research . . . . . . . . . . . 15

1.2 Research Objectives . . . . . . . . . . . . . . . 16

2 New Zealand AR Climatology 19

2.1 Data and Methods . . . . . . . . . . . . . . 20

2.1 .1 AR Detection . . . . . . . . . . . 20

2.2 AR Frequency and Seasonality . . . . . . . . . . . 27

2.3 AR Characteristics . . . . . . . . . . . . 37

2.4 Synoptic Types . . . . . . . . . . . . . . . . . . . . 41

2.4.1 Synoptic AR Climatology . . . . . . . . . . . . . 44

2.5 Conclusions . . . . . . . . . . . . . . . . . . 49

3 Impact of ARs on Precipitation $\quad 51$

3.1 Data and Methods . . . . . . . . . . . . . . . . 56

3.1 .1 Daily Rainfall Data . . . . . . . . . . . . . . . 56

3.2 New Zealand Rainfall . . . . . . . . . . . . . . . . . . . . 59

3.3 AR Impacts . . . . . . . . . . . . . . . . . . . . 61

3.4 Extreme Events . . . . . . . . . . . . . . . 65

3.5 Conclusions ..................... 75

4 Large-Scale Drivers of ARs $\quad 77$

4.1 Introduction . . . . . . . . . . . . . . 77

4.2 Data and Methods . . . . . . . . . . . . . 80 
4.3 SAM $\ldots \ldots \ldots \ldots \ldots \ldots \ldots$

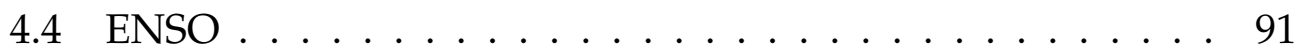

$4.5 \mathrm{IPO} \ldots \ldots \ldots \ldots \ldots$

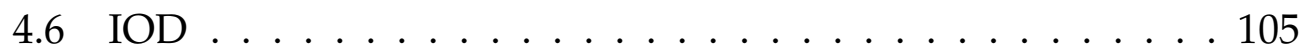

$4.7 \mathrm{MJO} \ldots \ldots \ldots \ldots \ldots \ldots \ldots \ldots$

4.8 Interaction between Climate Oscillations . . . . . . . . 115

4.9 AR Frequency Trend . . . . . . . . . . . . . . . . . . 116

4.10 Conclusions . . . . . . . . . . . . . . . . . 119

5 Conclusions $\quad 121$

$\begin{array}{ll}\text { Bibliography } & 125\end{array}$

6 Appendices 143

6.1 Appendix A . . . . . . . . . . . . . . . 143

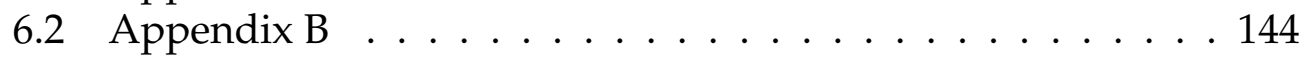

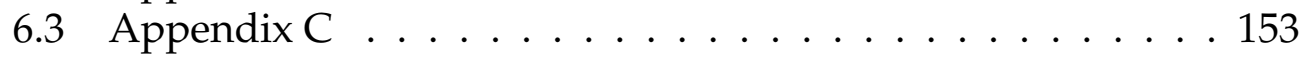

6.3.1 AR Case Study: March 2019 . . . . . . . . . . . . . 153

6.3 .2 Event Simulation . . . . . . . . . . . . . 153 


\section{Chapter 1}

\section{Introduction}

Atmospheric Rivers (ARs) are long, narrow jets of intense water vapour flux that are a fundamental component of the global atmospheric circulation, transporting moisture (and heat) from the tropics to higher latitudes. The first investigations of these elongated plumes of water vapour date back to at least the 1970s (Browning and Pardoe, 1973). Typically referred to as 'conveyor belts' in the field of synoptic meteorology, the term 'river' first appeared in the literature in the 1990s with the seminal work of Newell et al. (1992). A dedicated research field has since emerged, with a growing number of scientific publications discussing the climatology and impacts of 'atmospheric rivers'. The scope of AR research is extensive, involving the pure and applied fields of meteorology, hydrology, oceanography, atmospheric science, civil engineering, and water resource management. AR research has aided public and professional scientific communication related to natural hazards such as flooding, as well as the increasing threat of climate change (Ralph et al., 2017).

ARs form, intensify and decay with the natural passage of baroclinic systems across the mid-latitudes. When a cold front sweeps through the warm sector of the associated mid-latitude cyclone, a filament of high specific humidity forms in the pre-frontal region, which produces a strong poleward flow of water vapour within the warm conveyor belt (Zhu and Newell, 1994; Ralph et al., 2004). A typical AR transports a comparable water transport to that of the Amazon River (approximately $1.6 \times$ $10^{8} \mathrm{~kg} \mathrm{~s}^{-1}$ ) (Zhu and Newell, 1998). When an AR makes landfall, especially in areas of steep topography, it releases much of its water vapour as precipitation through orographic uplift. Thus, although ARs play a positive role in the distribution and maintenance of water resources in the midlatitudes, they are also associated with extreme precipitation and flooding. 
As global mean temperatures increase, the amount of moisture the atmosphere can hold (and ultimately release as precipitation) is increasing (Dessler et al., 2008). As a result, the frequency and intensity of extreme rainfall events is expected to increase in the future (Intergovernmental Panel on Climate Change, 2014; Reisinger et al., 2014). Therefore, an accurate understanding of the impacts and drivers of extreme precipitation is invaluable. In New Zealand, landfalling ARs have had major socioeconomic consequences, with losses to property, farmland, stock, roads and bridges (Reisinger et al., 2014; Frame et al., 2020; Reid et al., 2021). However, despite knowledge of their occurrence, focused investigations of ARs in New Zealand, including their physical and societal impacts, have received relatively little scientific attention (Kingston et al., 2016; Cullen et al. 2019; Little et al., 2019; Prince et al. 2021). In particular, little is known about how large-scale climate patterns (such as the Southern Annular Mode and El Niño-Southern Oscillation), which are known to significantly influence NZ weather and climate on daily to decadal timescales, influence ARs and AR-related precipitation extremes. The results presented in this thesis expand on previous research by focusing on the synoptic and large-scale climate drivers of ARs.

\subsection{Background}

\subsubsection{Atmospheric River Definition}

ARs can be identified within global fields of Integrated Total Column Water Vapour (IWV) and/or Integrated Water Vapour Transport (IVT). In terms of basic meteorological variables; IWV, eastward IVT (IVT $u$ ), northward IVT $\left(\operatorname{IVT}_{v}\right)$, and IVT magnitude (IVT) are calculated as:

$$
\begin{gathered}
\mathrm{IWV}=\frac{1}{g} \int_{p_{\mathrm{sfc}}}^{p_{\mathrm{t}}} q \mathrm{~d} p \\
\mathrm{IVT}_{u}=\frac{1}{g} \int_{p_{\mathrm{sfc}}}^{p_{\mathrm{t}}} q u \mathrm{~d} p \\
\mathrm{IVT}_{v}=\frac{1}{g} \int_{p_{\mathrm{sfc}}}^{p_{\mathrm{t}}} q v \mathrm{~d} p \\
\mathrm{IVT}=\sqrt{\mathrm{IVT}_{u}^{2}+\mathrm{IVT}_{v}^{2}}
\end{gathered}
$$

where $g$ is the gravitational acceleration, $u$ and $v$ are the layer-averaged (eastward and northward) horizontal wind speed components, and $q$ is the 
layer-averaged specific humidity. The integration is calculated between the surface $\left(p_{\text {sfc }}\right)$ and the top of the troposphere $\left(p_{\mathrm{t}}\right)$, typically between 500 $\mathrm{hPa}$ and $200 \mathrm{hPa}$.

(a)
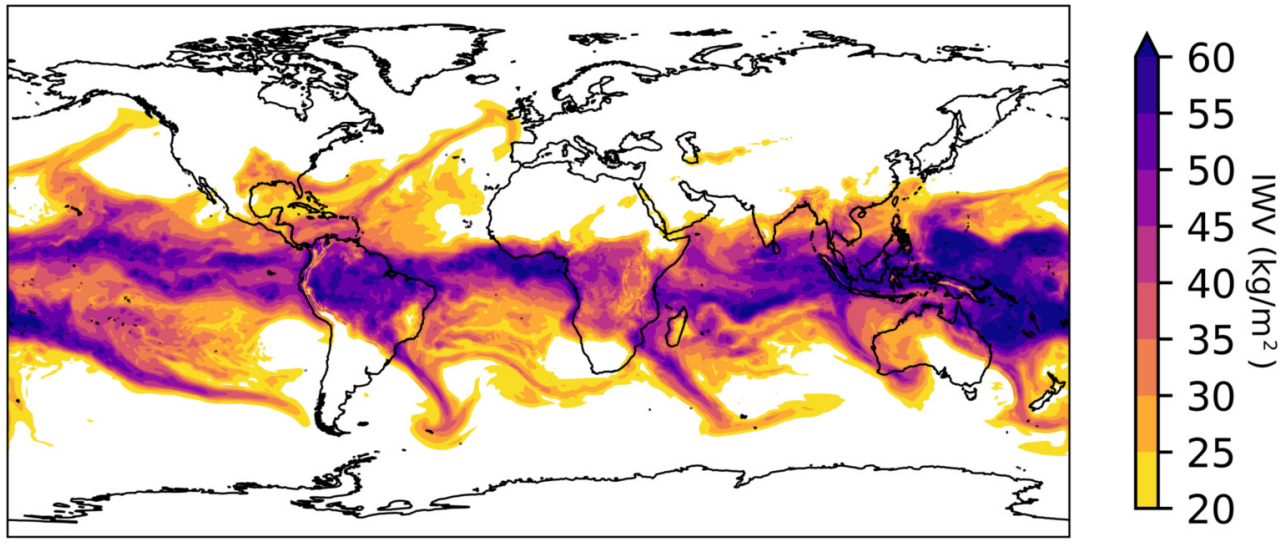

(b)
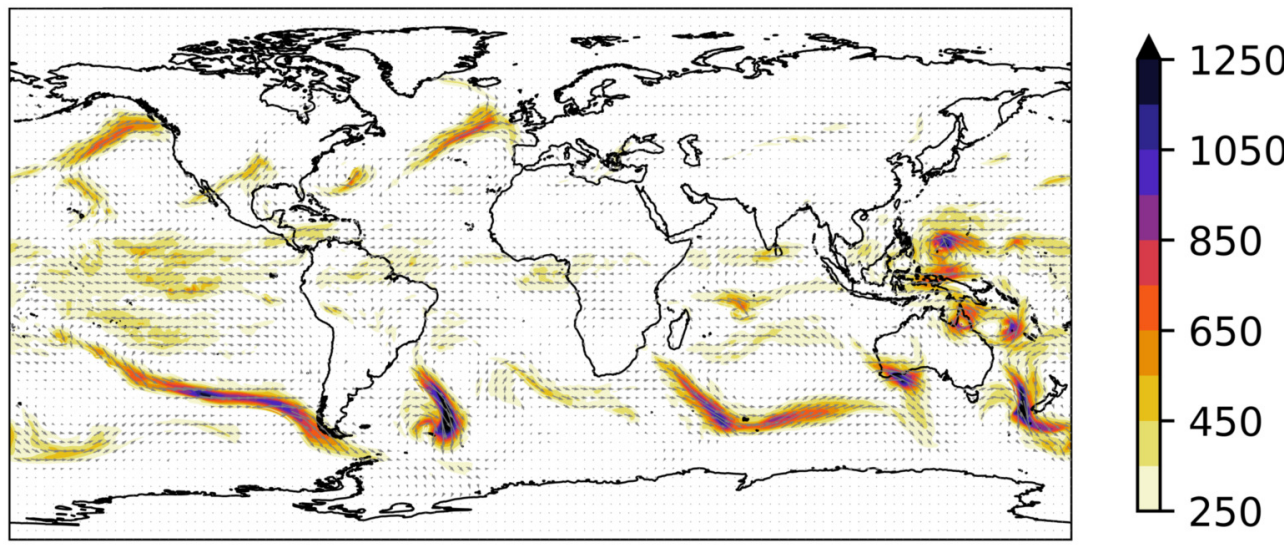

Figure 1.1: Snapshot of global fields of (a) Integrated Total Column Water Vapour (IWV) and (b) Integrated Water Vapour Transport (IVT), 2603-2018 1200 UTC. Several atmospheric rivers are evident; west of North America and Europe, off the coast of southern Chile, over the South Atlantic and Indian Oceans, and south of New Zealand.

Newell et al. (1992) first used the term 'tropospheric rivers' to refer to the filamentary structures observed in global fields of tropospheric water vapour flux, where the word 'river' was used to highlight the comparable flow rate with terrestrial rivers. Through the mid-to-late 1990s, the term 'atmospheric river' eventually became established in the literature 
following a number of influential papers (Zhu and Newell, 1994; Zhu and Newell, 1998). During the early 21st century, the AR definition was further refined, with ARs characterized as transient regions associated with the pre-cold front of mid-latitude cyclones, with length exceeding 2000 $\mathrm{km}$ and IVT above $250 \mathrm{kgm}^{-1} \mathrm{~s}^{-1}$ (Ralph et al., 2004). With an increasing number of publications, the new sub-field of AR science emerged. This culminated with the establishment of a regular International Atmospheric Rivers Conference in 2016, and publication of the first formal definition of an AR in 2017 (Ralph et al., 2017). The following definition was published in the Glossary of Meteorology of the American Meteorological Society:

"A long, narrow, and transient corridor of strong horizontal water vapour transport that is typically associated with a lowlevel jet stream ahead of the cold front of an extra-tropical cyclone. The water vapour in atmospheric rivers is supplied by tropical and/or extra-tropical moisture sources. Atmospheric rivers frequently lead to heavy precipitation where they are forced upward - for example, by mountains or by ascent in the warm conveyor belt. Horizontal water vapour transport in the mid-latitudes occurs primarily in atmospheric rivers and is focused in the lower troposphere. Atmospheric rivers are the largest 'rivers' of fresh water on Earth, transporting on average more than double the flow of the Amazon River."

(American Meteorological Society, 2021)

Prior to a formal definition, there was some confusion about the meaning of the term 'atmospheric river', with claims that it was duplicative of the existing concepts of the warm conveyor belt (WCB) and tropical moisture exports (TMEs). Although similar, each term has a distinct emphasis in the study of synoptic-scale water vapour transport, and their use is determined by the scope of the research aims. TMEs are defined as trajectories of intense horizontal moisture transport leaving the tropics, with objective identification of TMEs typically based on trajectory-based methods. ARs and TMEs are distinct, as ARs do not necessarily source moisture from the tropics, with the majority of ARs integrating moisture directly along their path through the mid-latitudes (Bao et al., 2006).

The WCB is characterized as the sector of dynamically uplifted heat and water vapour associated with an extra-tropical cyclone, where the majority of condensation and precipitation occurs. The WCB is typically identified as the coherent region where air ascends from the boundary layer to the upper troposphere along a constant isentropic surface (defined in 
a Lagrangian reference frame moving with the associated low-pressure system) (Carlson, 1980). ARs differ from WCBs as the emphasis is on horizontal water vapour transport, rather than the ascent forced by strong baroclinicity. Further, ARs may exist independently of individual midlatitude cyclones, and may remain quasi-stationary as they are enhanced by the formation of new cyclones (Dacre et al., 2019). Thus, ARs, TMEs and WCBs are distinct, and serve a complementary role in the conceptual model of moisture transport in the mid-latitudes. Following the above definitions, ARs can often be thought of as the pathways connecting TMEs to WCBs (a typical situation is shown in Figure 1.2).

\subsubsection{Atmospheric Circulation}

Atmospheric processes occur on a range of spatial and temporal scales and are interconnected; atmospheric processes at large spatial scales tend to occur over longer time periods, while processes at small spatial scales tend to occur over shorter time periods (Holton, 2004). In the study of ARs, we are primarily interested in the synoptic scale. The synoptic scale describes weather systems such as cyclones and anticyclones (and ARs), and corresponds to spatial scales on the order of thousands of kilometres, and time scales on the order of several days. As local-scale impacts can be understood in terms of synoptic-scale processes, ARs are especially useful for investigating high-impact events such as flooding. Thus, in bridging the gap between the large-scale circulation and local impacts, ARs serve as a useful tool. ARs are also useful for investigating the large-scale circulation, in particular the position and intensity of the storm track. Because the emphasis is on areas of high moisture transport (and individual high impact events) an AR climatology can reveal how the large-scale circulation influences the occurrence of extreme events (studies of other synoptic features do not resolve extremes events to the same extent). In this thesis we will proceed through a range of meteorological scales. In particular, we explore the synoptic circulation patterns associated with ARs (chapter 2 ), the local-scale impacts of ARs (chapter 3), and how ARs are influenced by the large-scale circulation (chapter 4 ).

The energy driving the global circulation of the atmosphere is provided by solar heating, which is unevenly distributed across the Earth due to latitude, season and land distribution. Preferentially warmed regions (i.e. the tropics) expand the air column, resulting in the same atmospheric 
(a)

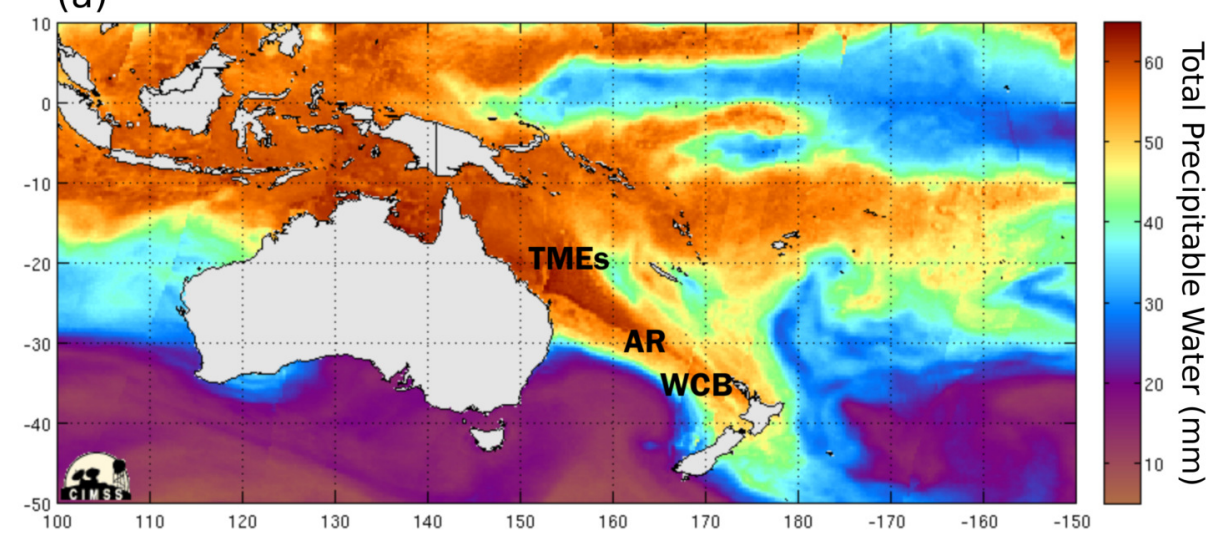

(b)

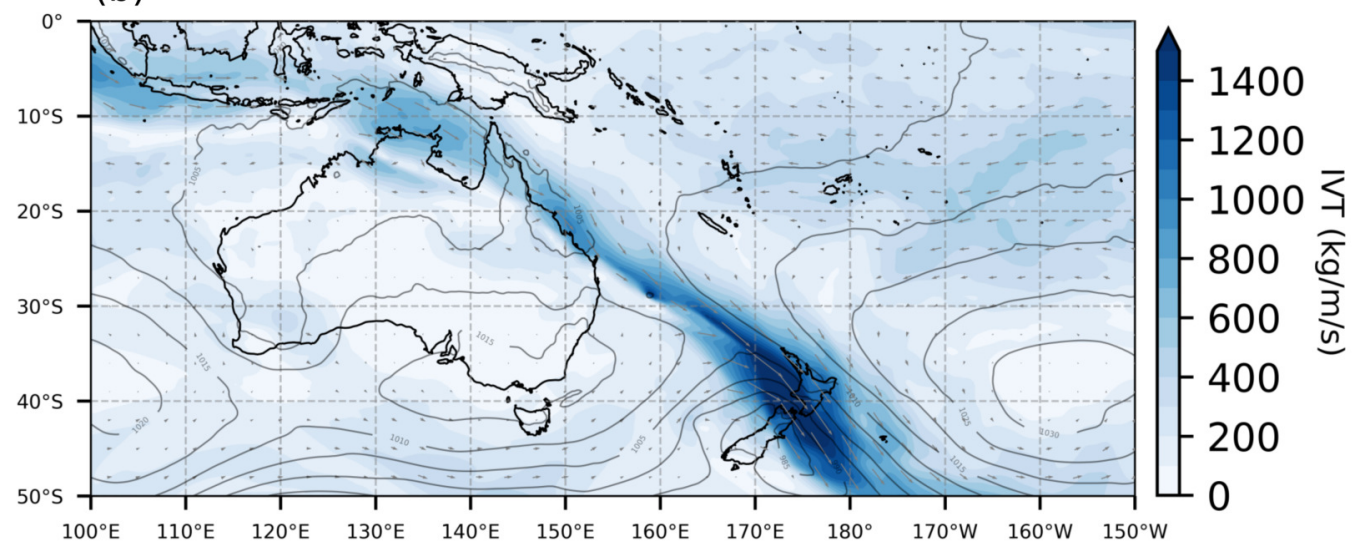

Figure 1.2: Atmospheric river making landfall in New Zealand, 27-1220101800 UTC. (a) SSMI/SSMIS/AMSR2-derived Total Precipitable Water, showing the approximate locations of the warm conveyor belt and tropical moisture exports. (b) ERA5 Integrated water vapour transport (filled contours and vectors) and sea level pressure (black contours). 
pressure located at a higher elevation. As a result, the pressure gradient force accelerates air at upper levels horizontally, from near the tropics toward the poles. The Coriolis force deflects horizontally moving air (to the left in the Southern Hemisphere), until a balance between the pressure gradient and Coriolis forces is reached. This results in a global-scale atmospheric circulation called the Hadley circulation, characterized by ascent near the equator, poleward flow at upper-levels, subsidence across the subtropics and mid-latitudes, and equatorward flow at lower-levels. The Hadley cell extends to approximately $30^{\circ}$ latitude, where the strong westerly flow drives the subtropical jet-stream. The subtropical jet-stream displays large seasonal variation, and is strongest during winter when the meridional temperature gradient reaches its maximum (Nakamura and Shimpo, 2004). Another jet-stream, the polar front jet stream, results from north-south temperature contrasts in the mid-latitudes (where strong upper-level westerly winds are driven by the 'thermal wind' relationship) (Holton, 2004). The position and intensity of the polar and subtropical jetstreams strongly influence storm track activity, and AR occurrence, across the mid-latitudes (Trenberth, 1991).

Superimposed upon the tropospheric jet stream, baroclinic waves develop due to instabilities in the large-scale flow. When the north-south temperature gradient is sufficiently large, potential energy of the mean state is converted to eddy kinetic energy, with the wavelength of maximum instability corresponding to the synoptic-scale (approx. 4000-5000 km) (Holton, 2004). Baroclinic instability is the primary mechanism for the development of mid-latitude cyclones (Holton, 2004). Baroclinic eddies play a crucial role in the climate system, transporting moisture, sensible heat, and angular momentum poleward, and limiting the temperature gradient across the mid-latitudes (Holton, 2004).

The majority of significant weather within mid-latitude cyclones is concentrated in narrow 'frontal zones', characterized as bands of strong horizontal temperature gradient a few tens of kilometres wide. When a cold front sweeps through the warm sector of the associated mid-latitude cyclone, a filament of high specific humidity forms in the pre-frontal region, which produces a strong poleward flow of water vapour within the warm conveyor belt (Zhu and Newell, 1994; Ralph et al., 2004). Provided the region of enhanced water vapour flux is sufficiently large in spatial extent, an atmospheric river will form ahead of the cold front. As the amount of moisture an air parcel can hold is dependent on its temperature, and temperature decreases with height (approx. $6.5^{\circ} \mathrm{C} / \mathrm{km}$ ), the majority of 
atmospheric moisture is held in the lower troposphere. Although upperlevel winds are much stronger, as the lower troposphere lies in contact with moisture sources and is warm enough to hold large amounts of water vapour, the majority of moisture transport occurs in the lower troposphere. Thus, approximately $75 \%$ of water vapour transport within an AR occurs in the lowest $2.5 \mathrm{~km}$ (Ralph et al., 2017). Upon ascent within the warm conveyor belt, condensation of water vapour results in the release of latent heat. Thus ARs transport a large amount of energy over long distances, from lower latitudes to higher latitudes, in the form of latent heat.

Atmospheric rivers are not uncommon. There are approximately 3-5 ARs present in each hemisphere at any given time (Zhu and Newell, 1998). ARs account for over $88 \%(84 \%)$ of total poleward water vapour transport in the Southern (Northern) Hemisphere, despite only covering 10\% of the mid-latitude zonal circumference (Guan \& Waliser, 2015). ARs are observed most frequently over mid-latitude ocean basins, and regularly make landfall on the west coasts of North America, southern South America, Europe, Greenland, southwestern Africa, Antarctica, Australia and New Zealand (Guan \& Waliser, 2015). Figure 1.3 shows the global frequency of AR occurrence. The corridors of highest AR frequency, located over the extra-tropical oceans, are aligned with the main mid-latitude storm tracks. The storm tracks emanate from regions of preferred cyclogenesis, where baroclinic instability of the mean flow, strong sea-surface temperature gradients, and strong upper-level divergence favour cyclone formation (Holton, 2004). In the Northern Hemisphere, regions of preferred cyclogenesis are located off the east coasts of Asia and North America (Whittaker and Horn, 1984). In the Southern Hemisphere, regions of preferred cyclogenesis are located off the eastern seaboards of South America and Australia, southeast of Africa, and near New Zealand (Sinclair, 1995).

\subsubsection{Atmospheric River Detection}

ARs are characterized as long and narrow filaments of intense moisture transport. Through application of an AR detection method, this qualitative definition can be made quantitative. A number of methods have been employed to automatically detect ARs from observations or model data. Early studies used integrated water vapour (IWV) as the main variable for AR detection, mainly as IWV data was available from satellite observations (Ralph et al., 2004; Wick et al., 2013). Eventually integrated water 


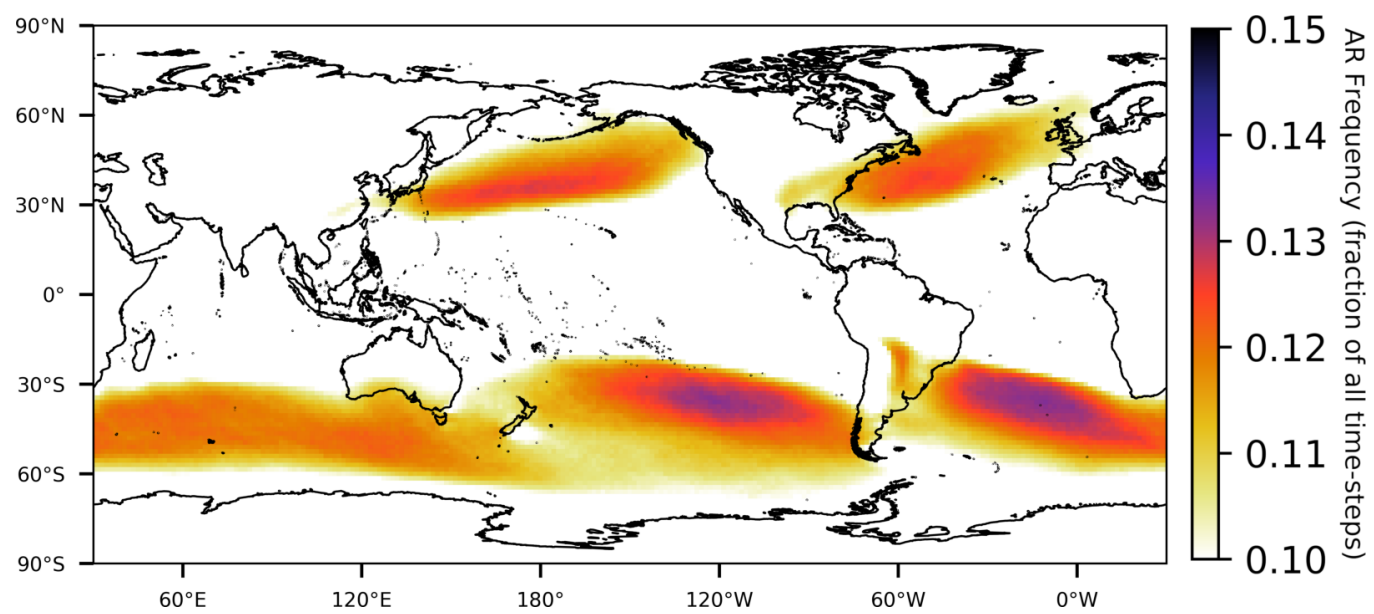

Figure 1.3: Frequency of atmospheric rivers globally, calculated as the fraction of all time-steps experiencing an AR. Derived from the global AR dataset of Guan \& Waliser (2015).

vapour transport (IVT) became the accepted detection variable, and the commonly used guideline threshold of IVT above $250 \mathrm{kgm}^{-1} \mathrm{~s}^{-1}$ was established (Ralph et al., 2004).

Compared to IWV (the total moisture in the air column), IVT (the flux of moisture) better fit the notion of ARs as regions of anomalous horizontal moisture transport. At higher latitudes, where total moisture is low but moisture flux can be high, detection techniques based on IVT are able to more reliably capture ARs. IVT is also directly related to orographic rainfall, as orographic precipitation amount is linearly proportional to the water vapour flux normal to the mountain barrier (Ralph et al., 2013). Further, Nayak et al. (2014) found that AR forecasts based on IVT were more skilful at longer lead times, compared with IWV.

To construct a spatially complete and long-term climatology of ARs, reanalysis datasets are often utilized. Typically, an AR detection algorithm using reanalysis data will search for regions of IVT above a certain threshold. The geometry of the identified region may also have to satisfy various other criteria (length, width, shape, orientation). Lavers et al. (2012) developed one of the first AR detection techniques using reanalysis IVT. This algorithm checks IVT magnitude against a threshold value across a pre-defined cross-section, and then checks adjacent grid cells against the threshold, searching away from the cross-section until a required length is reached. Later methods, such as the widely used global detection algo- 
rithm of Guan and Waliser (2015), introduced more advanced spatial and temporal criteria to detect ARs on both regional and global scales. Other novel algorithms make use of advanced mathematical techniques and machine learning (Pan and Lu, 2019; Muszynski et al., 2019). Despite a range of approaches, all detection methods can be characterized as having some intensity threshold (based on IWV or IVT, possibly time-varying) and geometry criteria (e.g. length, width, orientation). Many different detection algorithms have been applied in the literature, which has led to some confusion when comparing AR climatologies. The Atmospheric River Tracking Method Intercomparison Project (ARTMIP) is a recent effort to calculate uncertainties in AR research due to differences in detection method (Rutz et al., 2019).

\subsubsection{Global and Regional AR Studies}

Regional studies of ARs are primarily motivated by the wide-ranging impacts associated with these events, such as extreme precipitation, flooding, or snow/ice melt. The majority of regional AR studies have focused on the western coastlines of North America, where ARs provide $40-50 \%$ of annual precipitation (Dettinger et al., 2011; Ralph et al., 2013; Rutz et al., 2014), and are the primary cause of flooding events (Ralph et al., 2006). A number of studies have also investigated AR impacts in Europe (Lavers and Villarini, 2013), including Britain (Lavers et al., 2011), Norway (Azad and Sorteberg, 2017), the Iberian Peninsula and European Macronesian Islands (Ramos et al., 2015; Ramos et al., 2018). Recently, AR impacts have also been investigated in South America (Viale e al., 2018), South Africa (Blamey et al., 2018), Iran (Esfandiari and Lashkari, 2020), and East Asia (Kamae et al., 2017; Pan and Lu, 2020). In several regions, the term 'atmospheric river' has entered the mainstream lexicon. Non-technical names such as the 'Pineapple Express storm' (referring to ARs originating near Hawaii that make landfall in the western United States) have emerged. In the western United States especially, ARs are used to characterize, quantify, and communicate flood/drought risk for scientists, engineers, media, and policy-makers (Ralph et al., 2017). AR research has also extended into other applications such as avalanche risk (Hatchett et al., 2017), post-fire debris flows (Oakley et al., 2017), and dust transport (Ault et al., 2011).

The first comprehensive global study of ARs was conducted by Guan \& Waliser (2015), after developing a global-scale AR detection algorithm. Guan \& Waliser (2015) found AR landfall most frequent over mid-latitude ocean basins, with landfalls most common along the west coast of North 
America, southern South America, and Europe. Due to their association with mid-latitude cyclones, ARs are most frequent during winter for most regions (Waliser et al., 2012), and are strongly modulated by large-scale climate modes such as the El Niño Southern Oscillation (ENSO), MaddenJulian Oscillation (MJO), Arctic Oscillation (AO) and the Pacific-North American teleconnection pattern (PNA) (Guan and Waliser, 2015). Global studies of AR impacts on hydrological extremes show that ARs contribute $22 \%$ of mean annual global runoff, and can increase the occurrence of floods by $80 \%$ in regions of frequent AR activity (Paltan et al., 2017). These initial global AR studies have identified New Zealand as receiving a significant number of AR landfalls (Guan and Waliser, 2015), with notable hydrological impacts (Paltan et al., 2017). As New Zealand ARs have received significantly less scientific attention compared to other parts of the world, further research is required to fully understand the impacts and drivers of these events.

\subsubsection{New Zealand Climate}

The majority of New Zealand's precipitation falls in frontal systems associated with eastward-moving troughs, where rainfall is produced by uplift within the cold front (Tait and Fitzharris, 1998; Sturman and Tapper, 2006). Subtropical depressions crossing New Zealand from the north (often consisting of a complex pattern of warm and cold fronts) are also commonly associated with heavy rainfall, particularly in the North Island (Tait and Fitzharris, 1998; Sturman and Tapper, 2006). A significant number of New Zealand flooding events are associated with tropical cyclones that move into the mid-latitudes, transitioning into ex-tropical cyclones; on average approximately nine tropical cyclones form in the tropical southwest Pacific each year (typically between November and April), with approximately three migrating south of $35^{\circ} \mathrm{S}$ (with the highest fractions during March) (Sinclair, 2002). Settled weather in New Zealand is associated with anticyclonic conditions, which can persist for up to four weeks in summer (Sinclair, 1996).

New Zealand's topography (Figure 1.4), with mountain barriers almost perpendicular to the prevailing west/northwest flow, induce considerable extreme precipitation and flooding through orographic enhancement. Precipitation is strongly enhanced by the orography (on the windward side of mountain barriers), with orographic forcing of moist air over the Southern Alps producing 24-hour rainfall totals above $500 \mathrm{~mm}$ (Sturman and 


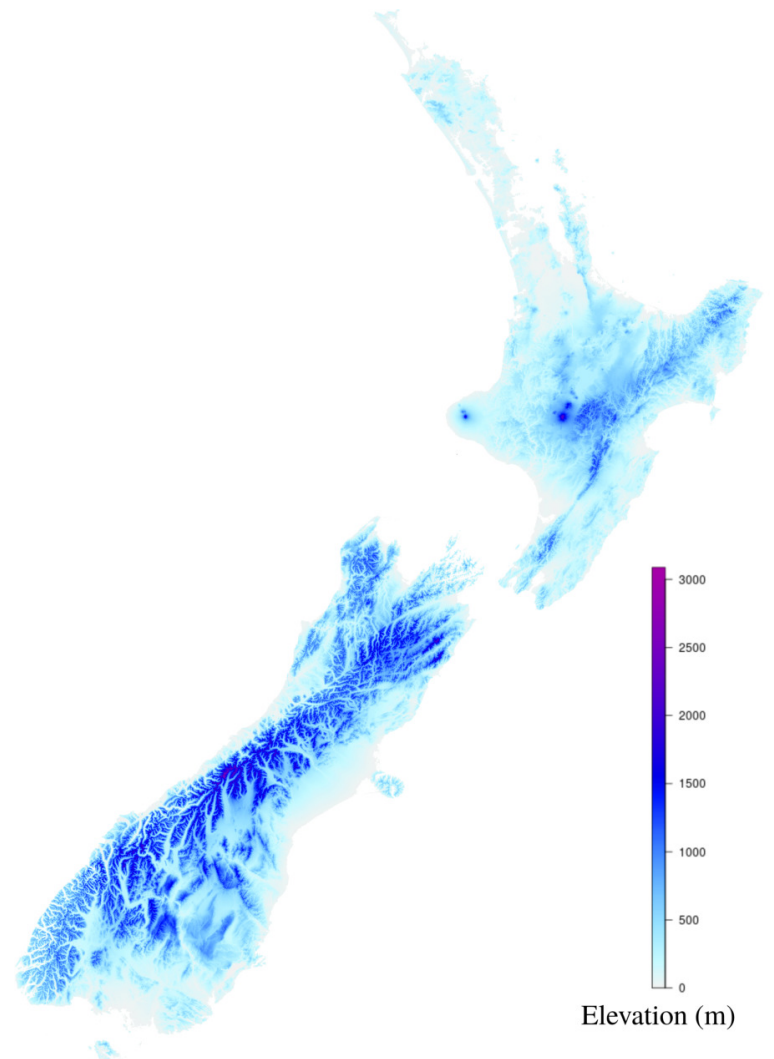

Figure 1.4: Orography of New Zealand as represented by the New Zealand $80 \mathrm{~m}$ Digital Elevation Model from the Shuttle Radar Topography Mission. 
Tapper, 2006). The Tararua, Ruahine, and Urewera ranges and peaks of the Central Volcanic Plateau also strongly influence the rainfall patterns of the North Island. Local-scale convective systems also contribute to rainfall totals; summer heating of the land and lower atmosphere can bring about sufficient convection to trigger sea-breeze convergence, cumulus development, showers and even thunderstorms, particularly in eastern regions (Tait and Fitzharris, 1998; Sturman and Tapper, 2006). The west coast of the South Island is New Zealand's wettest area, while the driest area is directly east of the divide. Annual rainfall totals range from below $500 \mathrm{~mm}$ at Alexandra, to above $3000 \mathrm{~mm}$ for elevated West Coast locations. The North Island and northern South Island receive the highest seasonal rainfall totals during winter, while the eastern South Island, south of Banks Peninsula, receives the highest seasonal rainfall total during summer. The west coast of the South Island receives the highest seasonal rainfall total during spring.

Regional precipitation in New Zealand is also influenced by the patterns of large-scale atmospheric circulation, and the organized modes of climate variability operating on various spatio-temporal scales (e.g. the Southern Annular Mode and El Niño-Southern Oscillation). These climate oscillations alter the storm track position and intensity as well as the mean pressure field across New Zealand, and influence the frequency of more structured objects such as ARs and atmospheric blocking highs. Previous investigations of New Zealand climate variables (e.g. precipitation, temperature, and soil moisture) have found significant correlations with the Southern Annular Mode (SAM), El-Niño Southern Oscillation (ENSO), Interdecadal Pacific Oscillation (IPO), Indian Ocean Dipole (IOD), and the Madden-Julian Oscillation (MJO) (Mullan, 1995; Salinger and Mullan, 1999; Salinger et al., 2001; Griffiths, 2007; Griffiths, 2011; Renwick, 2011; Fauchereau et al., 2016). For New Zealand, the interaction between each climate oscillation is complex, and further influenced by the seasonal cycle (Salinger and Mullan, 1999). In Chapter 4, we will describe the impacts of these large-scale climate oscillations on New Zealand ARs.

\subsubsection{New Zealand AR Studies}

New Zealand should experience appreciable impacts from ARs due to its location near the mid-latitude storm track combined with its steep, mountainous terrain which locally enhances precipitation rates. Indeed, global investigations of AR frequency have acknowledged New Zealand as receiving a significant number of AR landfalls (Guan and Waliser, 2015). 
Despite knowledge of their occurrence, focused investigations of ARs in New Zealand have been limited.

The first studies to explicitly mention AR impacts in New Zealand focused on flooding and glacier mass balance at specific South Island locations. An investigation of IVT and atmospheric circulation concurrent with major floods on the Waitaki River found that of the largest eight winter maximum floods between 1979 and 2012, all were associated with ARs (Kingston et al., 2016). These ARs were located in slow eastward moving mid-latitude cyclones, with high pressure to the northeast of New Zealand. A 2019 study found that ARs are responsible for the highest extreme ablation and snowfall events at Brewster Glacier in the Southern Alps, although the study also identified extreme IVT events occurring outside of conventional AR spatial structures (Little et al., 2019; Cullen et al., 2019). ARs have also been associated with singular flooding events in Golden Bay (Dean et al., 2013) and Northland (Rosier et al., 2015). In a global investigation of the contribution of AR variability to flood hazard and water availability, Paltan et al. (2017) found that more than $50 \%$ of New Zealand's mean annual runoff and up to $80 \%$ of high and low flows in New Zealand river catchments are attributed to ARs, where the absence of ARs was found to significantly increase the frequency of drought events.

The first comprehensive climatology of New Zealand ARs was recently published in Prince et al. (2021). On the West Coast of the South Island, ARs were found to make landfall 40 days per year on average, and account for $78 \%$ of total precipitation and $94 \%$ of extreme precipitation. A fivepoint $\mathrm{AR}$ rank scale was used to characterize AR events, where rank $5 \mathrm{AR}$ events contributed to 3-day precipitation totals exceeding $1000 \mathrm{~mm}$ in the Southern Alps. Further, another recent study found that ARs contribute to more than $60 \%$ of the largest snowfall (snow depth increase $>90$ th percentile) events in the Southern Alps (Porhemmat et al., 2021). Reid et al. (2021) found that between seven and ten of top ten daily rainfall totals at eleven New Zealand stations were associated with ARs. Further, nine of the ten most costly floods in New Zealand between 2007 and 2017 were associated with ARs (Reid et al., 2021). The emerging field of AR research in New Zealand provides exciting opportunities to advance understanding of extreme weather events, with applications in flood forecasting and freshwater management. The ultimate long-term goal is to bring AR research in New Zealand up to speed with other highly-impacted regions. 


\subsubsection{Emerging Fields in AR Research}

Future flooding and drought events in New Zealand will be influenced by changes in the frequency and intensity of AR events. In particular, with increased availability of water vapour due to warmer atmospheric conditions, there is increased risk of stronger AR impacts. Thus, quantifying climate model projections of future AR frequency and intensity is an important emerging research focus. In order to accurately predict changes in AR behaviour, an understanding of their interaction with large-scale climate dynamics is essential (Payne et al., 2020). In a review of AR research, Gimeno et al., (2014) identified that "the connection between occurrence and activity of ARs and large-scale ocean-atmosphere dynamics is very poorly understood". Likewise, in a review of the responses of ARs to climate change, Payne et al. (2020) highlights the fact that "at the global scale, much is still unknown about atmospheric rivers, including ...their interaction with large-scale dynamics". Therefore, within the AR science community, there has been a recent drive to understand the role of largescale ocean-atmosphere dynamics (e.g. Guan et al., 2013; Lavers and Villarini, 2013; Mundhenk et al., 2018), particularly how this knowledge may be used to benefit subseasonal-to-seasonal ${ }^{1}$ forecasting of ARs (Baggett et al., 2017; DeFlorio et al., 2019).

Given the large socio-economic impacts of weather hazards, accurate subseasonal to seasonal forecasts of extreme weather are essential, with important applications for operational forecasting and water resource management (DeFlorio et al., 2019). In particular, given the strong link between AR occurrence and regional precipitation, quantifying the spatio-temporal variability of AR occurrence, and the limits of AR prediction skill is essential. Wick et al. (2013) investigated the ability of ensemble forecasts to accurately predict AR landfalls in the Northeast Pacific. The models were able to forecast the occurrence of an AR at lead times of up to 10 days, though failed to accurately predict the timing and position of AR landfall. A number of other studies have investigated the prediction skill of IVT and AR forecasts on timescales of several days (Nayak et al., 2014; Lavers et al., 2014; Lavers et al., 2016), and have shown that AR forecast skill is sensitive to various climate oscillations (DeFlorio et al., 2019). Importantly, AR moisture flux (i.e. IVT) is found to be more predictable than AR-related impacts (i.e. precipitation) (Lavers et al., 2016). Thus, there is significant potential for the AR model to be applied in skilful forecasts of extreme precipitation.

\footnotetext{
${ }^{1}$ Forecasts on 1-5 week time-scales.
} 
Due to the chaotic nature of the climate system, the prediction of ARs on time-scales more than a few days is inherently very difficult. However, through linking ARs to large-scale climate modulations (such as the Madden-Julian Oscillation and El Niño Southern Oscillation), it is possible to improve the forecast skill of AR predictions (Guan et al., 2013; DeFlorio et al., 2019). In the Northern Hemisphere, there is a significant effort to quantify the subseasonal-to-seasonal prediction skill of ARs, and the role of large-scale ocean-atmosphere dynamics. For example, Baggett et al. (2017) found that a combination of phase 7-8 MJO (see section 4.7), and easterly quasi-biennial oscillation (QBO) improve AR prediction skill at lead times of two weeks. Mundhenk et al. (2018) identified similar linkages between AR occurrence and the MJO and QBO, which were able to provide skilful predictions of anomalous AR activity. Such investigations provide important "forecasts of opportunity" at lead times of one week to one month, which are able to provide important guidance (for decision makers and resource managers) on the probability of AR-related impacts (DeFlorio et al., 2019). In New Zealand, there is significant potential for $\mathrm{AR}$ research to improve long-range forecasts of extreme events. However, New Zealand remains an understudied region with regard to ARs, their impacts, and what drives them. In particular, how large-scale modes of climate variability influence AR behaviour in the Southwest Pacific region is not yet known. This thesis aims to fill these gaps, and further AR research in New Zealand, by focusing on the synoptic and large-scale climate drivers of ARs.

\subsection{Research Objectives}

While ARs have received extensive study in western North America and Europe, comparatively little is understood about the impacts of ARs in New Zealand. We further this emerging field by investigating the synoptic and large-scale drivers of ARs in New Zealand. The research objectives can be summarized as:

- Develop an atmospheric river detection algorithm for New Zealand.

- Investigate the frequency, seasonality and variability of ARs in New Zealand.

- Investigate the synoptic conditions and characteristics of landfalling ARs associated with extreme precipitation. 
- Investigate the impacts of ARs on NZ rainfall and flooding events.

- Investigate how climate oscillations such as the Southern Annular Mode (SAM) and El Niño Southern Oscillation (ENSO) influence AR frequency and severity.

The above objectives are explored through four main chapters. We have provided a brief overview of AR science and the climate of New Zealand (Chapter 1). Next, we investigate the general climatology of ARs in New Zealand, using two comprehensive AR datasets spanning the 1979-2019 period (Chapter 2). To better understand the synoptic conditions associated with ARs in New Zealand, we present the first investigation of Kidson synoptic types and atmospheric rivers. Then, we investigate how ARs influence precipitation and flooding events in New Zealand, using a daily rainfall dataset comprising 189 stations (Chapter 3). Finally, we provide the first focused investigation of the large-scale climate drivers of ARs in the severely understudied southwest Pacific region (Chapter 4). This study provides an in-depth overview of the drivers and impacts of ARs in New Zealand (an understudied region), further adding to the global survey of ARs. The results presented in this thesis give insight into the prediction of extreme rainfall events, and provide a basis for future investigations of ARs in New Zealand. 


\section{Chapter 2}

\section{New Zealand AR Climatology}

New Zealand, located in the southwestern Pacific Ocean between $34-47^{\circ} \mathrm{S}$ latitude, experiences a temperate maritime climate. The Southern Alps, rising over $3000 \mathrm{~m}$ high, are a prominent feature and produce significant climate variations between the east and west coasts of the South Island. New Zealand's rainfall is driven by the passage of frontal systems over the country, associated with eastward moving extratropical cyclones, about one cyclone center every 6-7 days (Sinclair, 1995). New Zealand should experience appreciable impacts from ARs due to its exposure to the prevailing westerlies, and steep topography. Indeed, global investigations of AR frequency have acknowledged New Zealand as receiving a significant number of AR landfalls (Guan and Waliser, 2015). Despite knowledge of their occurrence, focused investigations of ARs in New Zealand have received relatively little scientific attention.

Since the introduction of the AR concept, the majority of research has focused on ARs making landfall in the Northern Hemisphere, more specifically the western United States (Gimeno et al., 2014; Ralph et al., 2017). A few local AR climatologies have been constructed for Southern Hemisphere regions, including South Africa (Blamey et al., 2018) and South America (Viale et al., 2018). Recently, using the global AR dataset of Guan \& Waliser (2015), Prince et al. (2021) constructed the first climatology of ARs in New Zealand. ARs were found to make landfall on up to $11 \%$ of days annually, exhibiting a prominent seasonality, with a $61 \%$ increase in AR occurrence during the warm months (ONDJFM).

In this chapter we investigate the frequency, seasonality and characteristics of landfalling ARs. We extend the analysis of Prince et al. (2021), by developing and employing a new AR dataset over the 1979-2019 period. 
Further, we build on previous analysis by investigating the relationships between ARs and Kidson weather types, to better understand the synoptic conditions that caused each AR event. In Chapter 4, we investigate how AR occurrence is influenced by various large-scale climate oscillations. This climatology therefore provides a basis from which we assess AR-related impacts and the influence of large-scale climate variability.

\subsection{Data and Methods}

\subsubsection{AR Detection}

In the first climatology of New Zealand ARs, Prince et al. (2021) employed the global AR dataset of Guan \& Waliser (2015). We expand on this analysis by developing and employing our own AR detection algorithm (adapted for the New Zealand case). Throughout this thesis we will use two different AR detection methods; both the well-known global-scale algorithm of Guan \& Waliser (2015), and a new detection algorithm developed specifically for ARs making landfall in New Zealand. We will briefly describe the two detection algorithms below:

\section{Global AR Catalogue}

The global AR dataset used in this study was developed by Bin Guan, and is publicly and freely available online at https://ucla.box.com/ ARcatalog. The development of this AR detection algorithm and databases was supported by the National Aeronautics and Space Administration (NASA). The algorithm was originally introduced in Guan and Waliser (2015), refined in Guan et al. (2018), and further enhanced in Guan and Waliser (2019) with tracking capability. For our purposes, this dataset is used to compare with previous AR studies in New Zealand (Prince et al., 2021), as well as previous AR studies from other regions, and to assess AR activity over the larger southwest Pacific domain. This algorithm uses ERA-Interim reanalysis data (Dee et al., 2011) at $1.5 \times 1.5$ degree spatial resolution, and searches 6-hourly time-steps between Jan-1979 and Aug2019. AR detection is based on the following criteria:

(1) IVT intensity: IVT must exceed the 85th percentile at each grid cell. A lower limit of $100 \mathrm{kgm}^{-1} \mathrm{~s}^{-1}$ is also imposed.

(2) IVT direction: Mean AR IVT must be within $45^{\circ}$ of the AR shape orientation, with a poleward component $>50 \mathrm{kgm}^{-1} \mathrm{~s}^{-1}$, to ensure a poleward IVT transport in the direction of elongation. Further, no more than half 
of all grid-cells may deviate from the objects mean IVT direction by over $45^{\circ}$, to ensure coherence in IVT direction.

(3) Geometry: AR length must be greater than $2000 \mathrm{~km}$ and the AR must have a length-to-width ratio greater than 2.

(4) In the case that the 85th percentile contour is not well structured but there exists an AR-like inner core, steps (1) - (3) are repeated 5 times if (2) or (3) fails, each time increasing the IVT threshold applied in (1) by 2.5 th percentile.

Further details are provided in Guan and Waliser (2015). ARs that overlap the New Zealand land mask, provided the IVT direction at landfall is directed onshore, are defined as landfalling ARs. We consider each 6-hr time step where AR conditions are present over the landfall domain as a single AR. The characteristics of each AR, including length, width, mean IVT magnitude, mean IVT direction, landfall location, and the IVT magnitude and direction at landfall, are also recorded. At any instant in time, the locations that fall within the AR region are defined to be under AR conditions. Further, we define an AR event as a prolonged period of AR conditions, where two events are considered distinct if they are separated by more than 24 hours. The duration of an event is defined as the difference (in hours) between the first identified time-step and the last identified time-step comprising the event. During the Jan-1979 to Aug-2019 period, corresponding to 59,412 six-hourly time-steps, a total of 4,605 landfalling ARs in New Zealand were identified ( $7.8 \%$ of time steps), corresponding to 1,611 events.

\section{New Zealand AR Catalogue}

We are motivated to develop and apply a new detection method, which we will refer to as the 'New Zealand detection algorithm' (Kennett, 2021), specifically tailored for landfalling ARs in New Zealand (the python code for this algorithm is avalable at https://github.com/daemonkennett/ar_detection). We develop a second AR detection algorithm to further assess AR variability in New Zealand, and compare with the established dataset of Guan \& Waliser (2015). In particular, we aim to correct a number of the disadvantages associated with using the global AR catalogue for New Zealand. Our algorithm provides output at six-times higher spatial resolution compared to the global dataset, providing better spatial representation of landfall impacts. Further, we found that the global detection algorithm failed to detect a number of strong events in New Zealand, especially ARs with complex geometries. Indeed, the combination of a low IVT threshold (such as the commonly used $250 \mathrm{kgm}^{-1} \mathrm{~s}^{-1}$ ) and restrictive geometric conditions, such as the criteria implemented by Guan \& Waliser (2015), can cause the algorithm to miss the strongest AR events (Reid et al., 2020). For this reason, Reid et al. (2020) suggests an IVT threshold of between $350-500 \mathrm{kgm}^{-1} \mathrm{~s}^{-1}$. In developing the New Zealand detection algorithm, we tested a number of IVT 
thresholds (including new AR detection variables, see Appendix A) and geometry constraints. Of the conditions tested, it was decided to use a higher IVT threshold (95th percentile IVT) compared to the global dataset (85th percentile IVT), to focus on the most intense events. In addition, use of a higher IVT theshold better fits the notion of ARs as 'extreme' events (as opposed to occuring within the majority of frontal systems over the country, whether posing significant flood risk or not). See Figure 2.1 for a comparison of IVT thresholds used in the global and NZ detection algorithms. A higher IVT threshold results in fewer ARs detected, as it excludes the weakest ARs. The weakest ARs, with max IVT less than $500 \mathrm{kgm}^{-1} \mathrm{~s}^{-1}$ are described by Ralph et al. (2019) as 'weak and primarily beneficial', and correspond to Category 1 AR events. Although the New Zealand detection algorithm may exclude some of these low IVT AR events, we are primarily interested in AR-related extreme rainfall and flooding, thus our algorithm is deemed appropriate for our purposes. The New Zealand detection algorithm provides the primary AR dataset used throughout this thesis, which we complement and compare with the global AR catalogue. By including both datasets in our analysis, we capture both the weakest and most extreme AR events, and minimise any bias arising from the AR detection method (namely the IVT threshold) used. Throughout the thesis, we will explicitly mention the AR dataset that is used in the analysis.

For our detection method, identification of ARs is based on the intensity and geometry of integrated water vapour transport (IVT). This data is sourced from the ERA5 reanalysis dataset of the European Centre for Medium-Range Weather Forecasts (Hersbach et al., 2020). We retrieve data at six-hourly time steps, with a grid resolution of $0.25^{\circ} \times 0.25^{\circ}$. The IVT magnitude and direction $(\theta)$ are derived from the vertical integrals of eastward and northward water vapour flux $\left(\mathrm{IVT}_{u}\right.$ and $\left.\mathrm{IVT}_{v}\right)$. The ERA5 reanalysis dataset was chosen due to its high spatial resolution and detailed representation of atmospheric water-vapour (Wang et al., 2020; Hersbach et al., 2020). Nevertheless, there is good agreement in AR detection between different reanalysis products (Lavers et al., 2012; Guan and Waliser, 2015). We employ reanalysis data at instantaneous 6-hourly time-steps, consistent with the majority of previous AR climatologies. A sub-daily temporal resolution is used as ARs and AR-related impacts are often short in duration, occurring on time-scales less than 24 hours. As the largest sensitivity in AR detection is due to spatial resolution, rather than temporal resolution (Guan and Waliser, 2015), we do not expect significant differences in algorithm output due to the size of the time-steps.

The New Zealand detection algorithm uses 6-hourly IVT data from the ERA5 reanalysis dataset with a grid resolution of $0.25 \times 0.25$ degrees, to detect ARs between Mar-1979 and Feb-2020 ${ }^{1}$. The algorithm identifies regions of enhanced IVT

\footnotetext{
${ }^{1}$ Due to a lack of observational data, quality reanalysis products are not available for
} 
whose shape and IVT direction are consistent with the AR definition. At any instant in time, the locations that fall within this region are defined to be under AR conditions. To identify regions of enhanced IVT, the IVT magnitude is checked against a threshold value. We use a higher IVT threshold compared to Guan \& Waliser (2015) to focus on the strongest events. The threshold value is defined monthly for each grid cell as the 95th percentile IVT during the 5-month period centred on that month. Using a time-varying threshold removes the influence of the seasonal cycle. A fixed lower limit of $250 \mathrm{kgm}^{-1} \mathrm{~s}^{-1}$ is also imposed.

Contiguous regions of grid cells with IVT values above the percentile threshold and fixed lower limit are isolated, and labelled. If a region of enhanced IVT intersects the New Zealand landfall domain, the grid cell with the maximum IVT over the landfall domain is identified. This grid cell is defined as the landfall location. The object axis is then calculated following Brands et al. (2017). Axis calculation can be described as follows:

(1) The landfall location is labelled as the target grid cell, $e$.

(2) The IVT direction at $e$ is calculated and discretized into one of 8 cardinal directions $(\mathrm{N}, \mathrm{NE}, \mathrm{E}, \mathrm{SE}, \mathrm{S}, \mathrm{SW}, \mathrm{W}, \mathrm{NW})$. Of the 8 grid cells adjacent to $e$ the upstream grid cell $s$ and the two grid cells neighboring $s$ are identified. Of these 3 candidate grid cells, the one with maximum IVT is tested to determine if the IVT exceeds the percentile threshold.

(3) If the IVT threshold is exceeded this grid cell is labelled as the new target grid cell $e$, and we repeat step (2). This process is continued until the upstream grid cell fails to exceed the threshold or a grid cell is detected twice.

The grid cells identified by this process comprise the object axis. The length of the object is computed as the sum of the distances between neighbouring axis cells.

To be identified as an AR, the following geometry criteria must be satisfied:

Length Check: The length of the object must exceed $2000 \mathrm{~km}$. This value was established by (Ralph et al., 2004) and has since been widely used.

Narrowness Check: The width of an object is defined as its surface area divided by its length. An object is discarded if its length/width ratio is less than 2 .

Mean Meridional IVT Criterion: An object is discarded if the mean IVT does not have a poleward component greater than $50 \mathrm{~kg} / \mathrm{m} / \mathrm{s}$. This filters objects that do not transport moisture toward higher latitudes.

the Southern Hemisphere before 1979 (prior to the satellite observing era). As AR detection relies on accurate representations of moisture and wind speed (at sufficiently high temporal resolution), our analysis of ARs is therefore limited to the period between 1979 and 2020. 


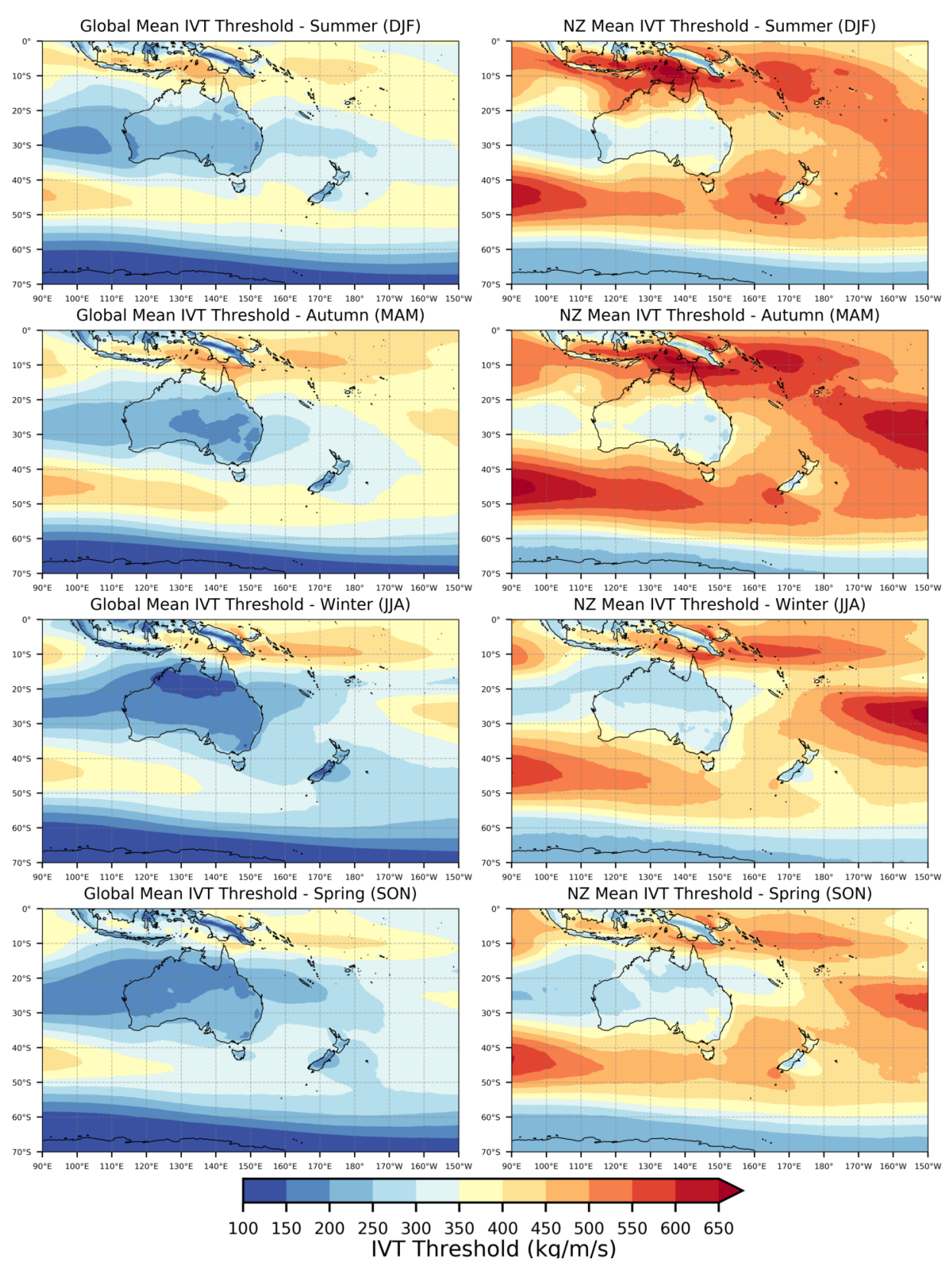

Figure 2.1: Comparison of the IVT threshold used in the global AR detection algorithm, 85th percentile IVT, (Guan \& Waliser, 2015) (left) and the New Zealand AR detection algorithm (Kennett, 2021), 95th percentile IVT, (right). Shown are mean thresholds for each season over the 1979-2020 period. 
Coherence in IVT Direction Criterion: An object is discarded if more than half of the grid cells have IVT deviating more than 45 degrees from the object's mean IVT. This filters objects that do not feature a coherent IVT direction.

By far the largest degree of filtering is associated with the length criteria. For the Mar-1979 to Feb-2020 search period, a total of 1,453,710 enhanced IVT objects were identified over the detection region. This is reduced to 19,465 objects that meet both the landfalling and length criteria. Of the objects that satisfy the length criteria, $3.5 \%$ did not meet the narrowness criteria. Of the remaining objects, $4.4 \%$ did not meet the mean meridional IVT criterion, and a further $1.6 \%$ did not meet the coherence in IVT direction criterion. The detection method is applied independently on each 6-hourly time-step, therefore AR counts are calculated as the number of individual AR time-steps, rather than singular AR occurrences. Throughout the analysis we therefore refer to 'AR frequency', calculated as the ratio of identified AR time-steps to the total number of time-steps. During the Mar-1979 to Feb-2020 period, corresponding to 59,904 six-hourly time steps, a total of 3,797 landfalling ARs in New Zealand were identified (approximately 6\% of time steps).

Again, we also define individual AR 'events' as continuous periods of AR conditions, where two events are considered distinct if they are separated by at least $24 \mathrm{~h}$. Further, an event is labelled as 'persistent' if it occurs for more than three time-steps $(>18 \mathrm{~h})$. These definitions are consistent with the existing literature (see for e.g. Lavers et al., 2012; Lavers and Villarini, 2013; Ramos et al., 2015). Event duration is defined as the difference (in hours) between the first identified time-step and the last identified time-step comprising the event. A total of 1,254 events were identified over the period, of which 514 (approximately 41\%) were classed as persistent.

Little et al. (2019) suggested that certain AR detection algorithms may underestimate the frequency of enhanced IVT events in New Zealand, due to the spatial complexity of IVT structures around New Zealand. We found that the majority of enhanced IVT events in New Zealand were captured, as both the Guan \& Waliser (2015) detection algorithm and the New Zealand algorithm have relatively relaxed constraints on AR shape. However, the Guan \& Waliser (2015) algorithm did fail to identify a number of recognized AR events, likely due to not meeting the geometry criteria. By using a higher IVT threshold, our method aims to ensure all significant AR events are correctly identified. While the geometry criteria applied by our algorithm are similar to that of Guan \& Waliser (2015), when combined with a higher IVT threshold, we are better able to capture enhanced IVT objects with more complex geometries as a smaller AR boundary is identified (Figure 2.2). Often the AR boundary outlined by the 85th percentile IVT threshold encompasses the entirety of New Zealand. Thus, another advan- 
(a) Atmospheric River Detected at 2016-11-14 06 UTC

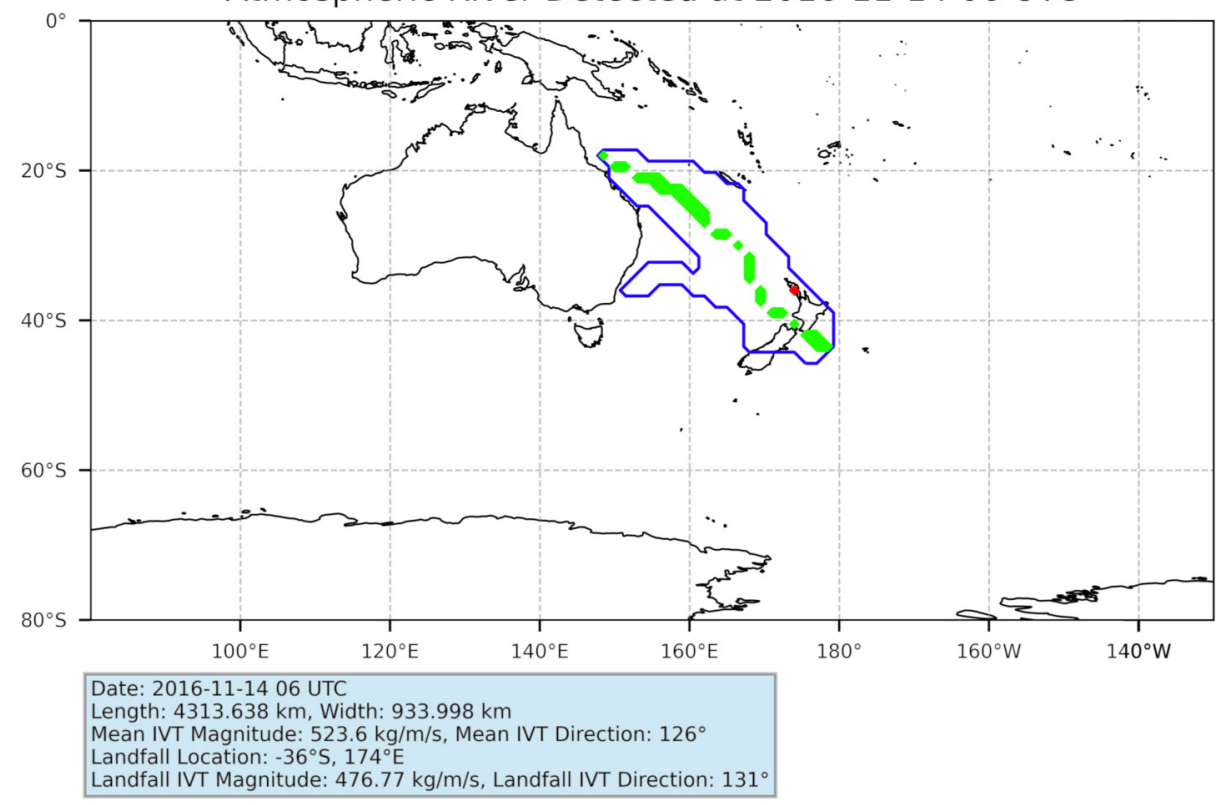

(b) Atmospheric River Detected at 2016-11-14-06 UTC

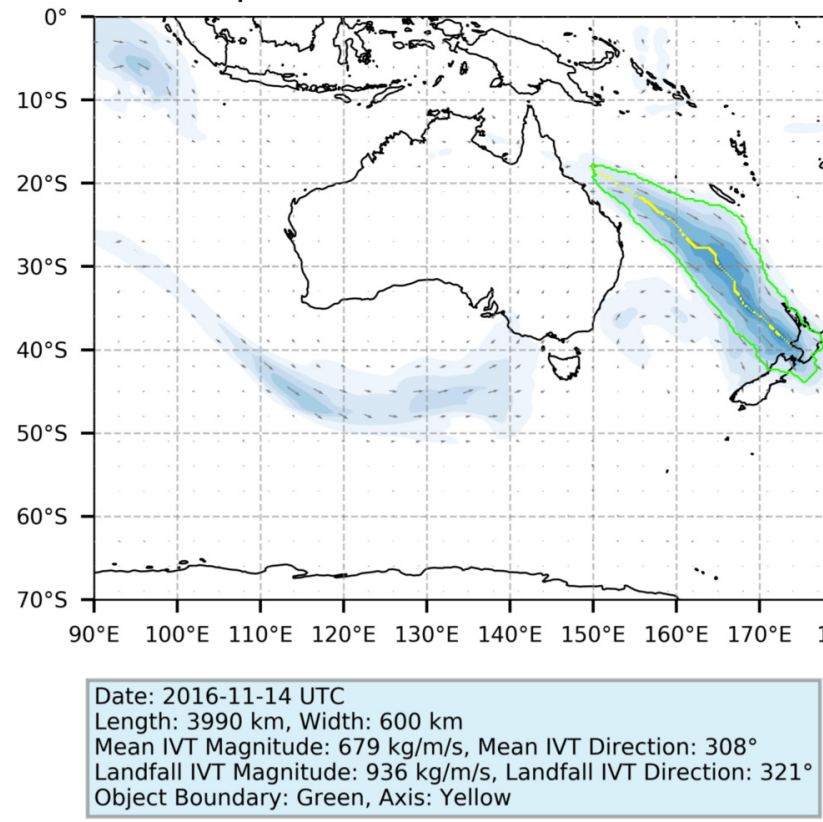

Figure 2.2: Example AR detection algorithm output for the (a) global dataset (Guan \& Waliser, 2015) and (b) NZ dataset (Kennett, 2020). Note the smaller AR region identified by the NZ detection algorithm. The AR (detected on 2016-11-14 0600 UTC) was associated with flooding for the Wellington region. 
tage of a higher IVT threshold is that it highlights the inner AR core where the strongest water vapour flux (and precipitation rates) occur, allowing us to better assess local impacts. In general, IVT values exceeding $750 \mathrm{kgm}^{-1} \mathrm{~s}^{-1}$ are considered notable. Values over $1000 \mathrm{kgm}^{-1} \mathrm{~s}^{-1}$ are considered extreme, and often indicate a significant flooding threat. Thus, use of a higher IVT threshold allows the AR boundary to represent an area of potentially hazardous conditions. Note that we also exclude the 'consistency between object mean IVT direction and overall orientation' criteria to allow for objects associated with more complex geometries that still fulfill the AR definition. Further, we do not require IVT to be directed inland for the AR to be classed as 'landfalling', so long as the AR shape intersects the New Zealand land mask. Note that although AR frequency is sensitive to IVT threshold values (hence the NZ AR catalogue identifies fewer events), the identified spatial patterns are consistent between the datasets.

\subsection{AR Frequency and Seasonality}

In the Southern Hemisphere, peak zonal-mean AR frequency occurs at approximately $41^{\circ} \mathrm{S}$ latitude, corresponding to the mean latitude of New Zealand (Guan \& Waliser, 2015). The median lowest latitudinal extent of ARs in the Southern Hemisphere is $29^{\circ} \mathrm{S}$, while the median highest latitudinal extent occurs at $50^{\circ} \mathrm{S}$ (Guan \& Waliser, 2015). Thus New Zealand lies directly in the centre of AR activity, with significant AR activity also to the north and south of the country. During the Jan-1979 to Aug-2019 period, corresponding to 59,412 six-hourly time-steps, a total of 4,605 landfalling ARs in New Zealand were identified ( $7.8 \%$ of time steps) by the global AR catalogue. For the NZ AR catalogue, operating at a higher IVT threshold, a total of 3,797 landfalling ARs were identified (approximately $6 \%$ of time steps) over the Mar-1979 to Feb-2020 period. Figure 2.3 shows the annual AR counts (including event and persistent event counts) between 1980 and 2019. Typically, between 60 and 120 landfalling ARs are identified each year.

The genesis location of cyclones in the Southern Hemisphere is seasonally dependent, related to the position of the jet stream and the regions of strongest SST gradient (Sinclair, 1995). Cyclones (and hence ARs) are observed to preferentially form in the Indian Ocean, the South Australian Basin and off the east coast of Australia. ARs of tropical origin are also observed, and can be associated with tropical and ex-tropical cyclones, though the majority of New Zealand ARs form, intensify and decay within the $30^{\circ}-60^{\circ} \mathrm{S}$ latitude band. There exists a strong relationship between the distribution of IWV/IVT and AR occurrence, therefore AR frequency is heavily dependent on the moisture content of the atmosphere (and changes in mean atmospheric temperature). Overall, on the global scale, warm season ARs are more common then cold season ARs. Over the entire Southern Hemisphere, on average approximately 6.9 ARs are present at any one time-step 
(a)

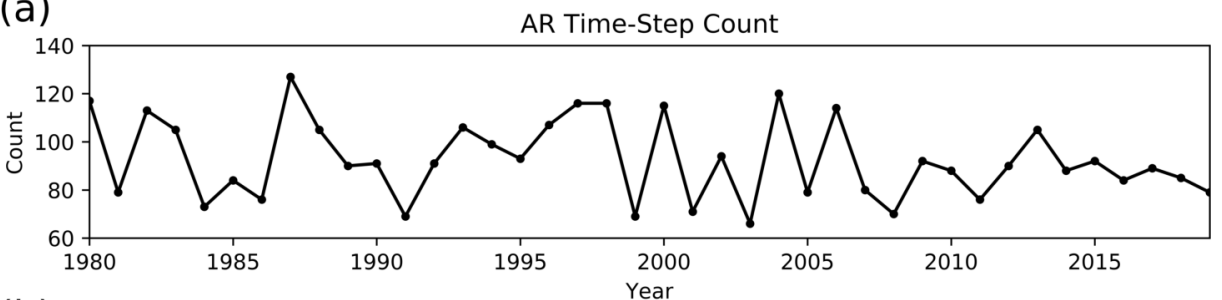

(b)

AR Event Count

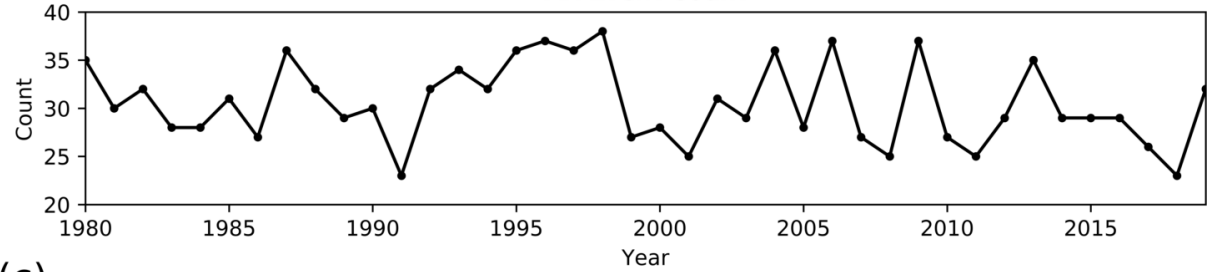

(c)

Persistent Event Count

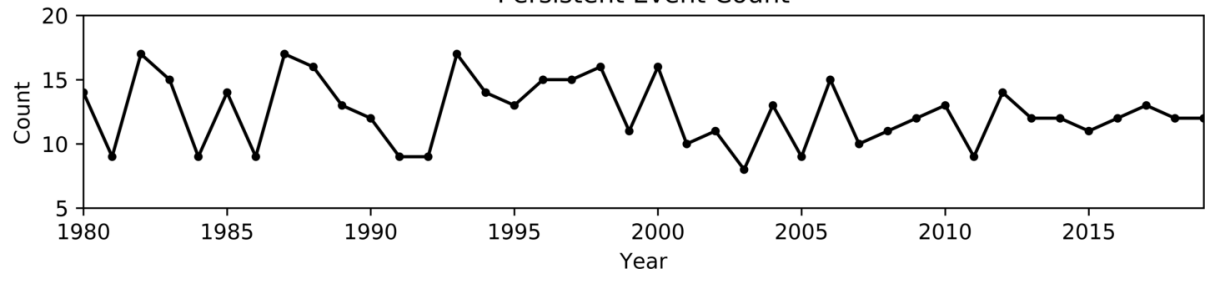

Figure 2.3: Interannual variability in AR occurrence showing the number of identified (a) AR Time-Steps, (b) AR Events and (c) Persistent AR Events between 1980 and 2019. Derived from the NZ AR dataset. 
during austral summer. While approximately 6.3 ARs are present at any one timestep during austral winter. In the Northern Hemisphere, on average 7.1 ARs are present at any one time-step during boreal summer, compared to 5.1 ARs during boreal winter. Thus, increased atmospheric moisture due to higher mean atmospheric temperatures appears to be a dominant driver of summer AR occurrence. Thus, AR seasonality in New Zealand is likely related to hemisphere-wide changes in AR occurrence, as well as latitudinal shifts in peak AR occurrence. Prince et al. (2021) highlighted the role of seasonal changes in the position of the polar and subtropical jet streams. Using the global AR dataset of Guan \& Waliser (2015), Prince et al. (2021) found a 50\% reduction in AR occurrence for southern regions during winter, primarily due to the influence of the winter split jet.

During austral summer, the lack of a developed subtropical jet results in the formation of a singular circumpolar storm track along the polar jet stream (Nakamura and Shimpo, 2004). During austral winter, the main upper-level storm track is located along the subtropical jet over the South Pacific, while a low-level storm track forms along the surface baroclinic zone off the coast of Antarctica (Nakamura and Shimpo, 2004). This results in a split jet structure near New Zealand during winter, which acts to suppress storm track activity in the South Pacific. The Southern Hemisphere split-jet at the longitude of New Zealand is a prominent feature of the climate during austral winter. As the jet stream shifts, so does the storm track, and associated baroclinic processes (Trenberth, 1991). Thus, the position of the jet stream strongly controls the eastward propagation of ARs, as it shapes the distribution of low-level moisture and winds, and thus IVT. In particular, the zonal symmetry of the storm track is greatest during summer, while storm track activity extends over a wider range of latitudes during winter (Trenberth, 1991). This feature produces a strong seasonality in AR frequency in New Zealand, distinct between the North and South Islands. The impact of the split jet on winter AR frequency in the Australia/New Zealand region is apparent in Figure 2.4, with high AR frequency to the north and south of New Zealand. In particular, we see that the region of peak AR frequency to the south of New Zealand is located further poleward during winter. During summer, the mean position of the polar jet is located nearer the equator, leading to a significantly higher number of AR landfalls.

The seasonal variation in New Zealand AR frequency is atypical, as ARs are typically driven by winter storms for most other regions where ARs are studied. In particular, extreme AR precipitation is usually attributed to winter events as the air is closer to saturation (Lavers et al., 2011; Neiman et al., 2011). Notable exceptions include the east coast of Asia (Pan and Lu, 2020), and the west coast of North America between $41^{\circ} \mathrm{N}$ and $45^{\circ} \mathrm{N}$ latitude, which experiences peak AR frequency in June (Rutz et al., 2019). The seasonal cycle in this region of North America was found to be significantly enhanced or completely offset by the El Niño-Southern 

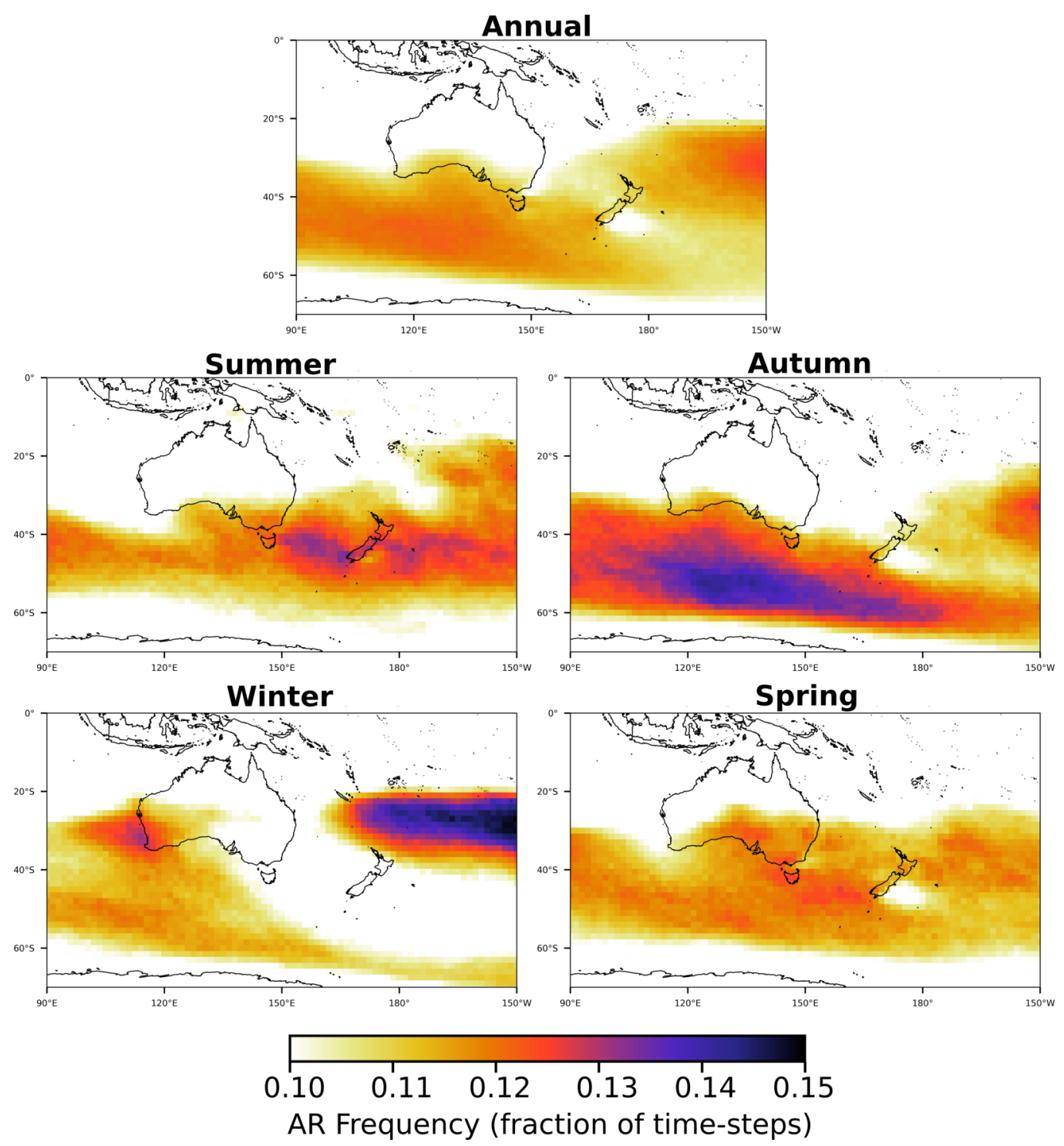

Figure 2.4: Annual and seasonal distribution of landfalling AR frequency derived from the global AR catalogue. 
Oscillation (ENSO) and the Madden-Julian oscillation (MJO) (Mundhenk et al., 2016). In Chapter 4, we explore how seasonal variation of New Zealand ARs is influenced by coupled ocean-atmosphere climate drivers. The seasonal AR frequency distribution for New Zealand shows similar patterns to South America (located at roughly the same latitude), with an equatorward shift in the location of peak AR frequency during winter (Viale et al., 2018). However, as New Zealand lies to the south of the zone of maximum winter AR frequency, it experiences comparatively fewer cold season ARs overall.

Figure 2.5 shows the seasonal AR frequency at approximately $30 \mathrm{~km}$ grid resolution. ARs make landfall most often on the west coast of the South Island, south of $44^{\circ} \mathrm{S}$. Off the coast of Fiordland, 16 days per year on average ( $4 \%$ of time-steps) occur under AR conditions. A clear rain shadow effect is observed, with AR conditions observed much less frequently east of the Southern Alps. As ARs interact with the topography, wind speeds can be reduced and moisture is lost through precipitation, thus enhanced IVT may not extend to the leeward side of the mountain barrier. Rutz et al. (2014) found that water vapour depletion upon interaction with a topographic barrier was a key contributor to AR decay, and inhibits the inland penetration of ARs in the western United States. Indeed, in the lee of the South Island, a significant reduction in AR frequency is observed extending to the date line.

AR landfalls in the South Island occur most often during spring and summer, and in the North Island during winter and spring. Overall, the September to January period is recognised as experiencing a higher frequency of AR landfalls. The higher winter frequency in the North Island likely reflects the increased cyclogenesis off the eastern seaboard of Australia during winter (Sinclair, 1995). The higher frequency of AR events in the South Island during warmer months is likely due in part to the more pronounced summer ridges providing stronger water vapour flux over a longer period. As southern regions are embedded in the westerly wind belt year round, the higher number of summer ARs is likely also due to a regional increase in the availability of moisture (and hence moisture flux) due to higher temperatures. Thus, it is hypothesized that although winter fronts may be more common, the extent of water vapour (or simply the magnitude of IVT) may not be sufficient to constitute an AR. Use of IVT as a detection variable may in fact underestimate the number of winter ARs, and winter AR impacts. The IVT thresholds used to detect ARs were defined monthly to account for seasonal variations in IVT. However, AR frequency may be influenced by the thresholding method, and more frequent summer ARs might be observed simply due to higher mean IVT in summer ${ }^{2}$. Indeed, the mean IVT of spring/summer ARs (634

\footnotetext{
${ }^{2}$ In developing the New Zealand detection algorithm, we trialled a new AR detection variable in an attempt to correct this problem, see Appendix A.
} 

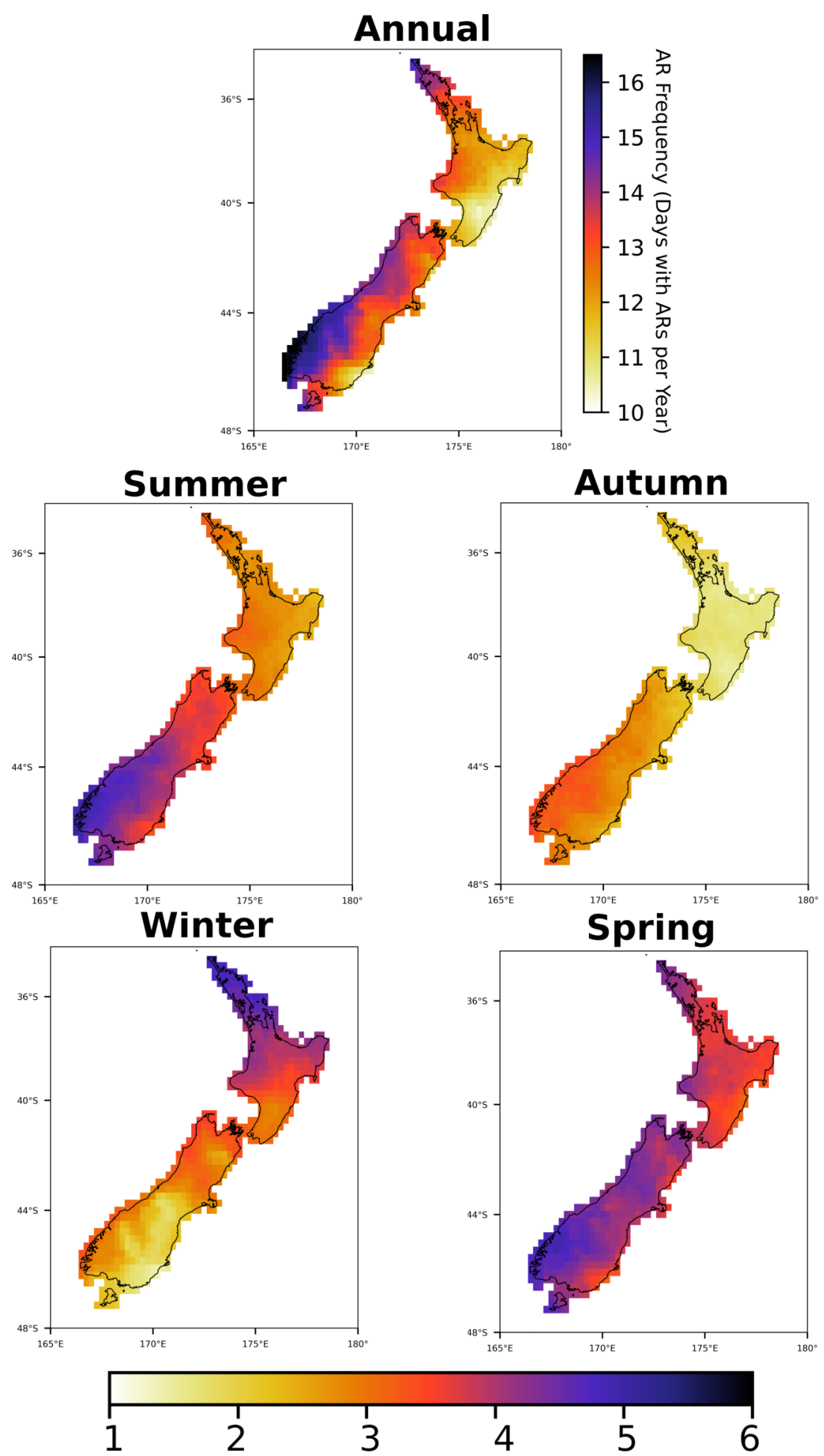

AR Frequency (Days with ARs per Year)

Figure 2.5: Annual and seasonal distribution of landfalling AR frequency over the New Zealand landfall domain, derived from the New Zealand AR catalogue. 
$\left.\mathrm{kgm}^{-1} \mathrm{~s}^{-1}\right)$ was significantly higher than autumn/winter ARs $\left(594 \mathrm{kgm}^{-1} \mathrm{~s}^{-1}\right)$. Mean IVT at landfall is also higher for summer ARs $\left(770 \mathrm{kgm}^{-1} \mathrm{~s}^{-1}\right)$ compared to winter ARs $\left(676 \mathrm{kgm}^{-1} \mathrm{~s}^{-1}\right)$.

Most landfalling ARs in New Zealand are associated with mid-latitude troughs, however many ARs are also directly or indirectly associated with tropical and ex-tropical cyclones, particularly north of New Zealand. A number of landfalling ARs source moisture from tropical cyclones (with cyclone centers contained within the AR boundary), hundreds of kilometres from the New Zealand mainland. For example, the highest daily rainfall total observed at Milford Sound in January 1994 was linked to tropical moisture from tropical cyclone Rewa, north of New Caledonia (Reid et al., 2021). The AR event affecting the North Island in April of 2017 (the second most costly flooding event between 2007 and 2017) transported moisture from tropical cyclone Debbie toward New Zealand (Reid et al., 2021). The AR that led to extensive flooding in Westland in March 2019 sourced warm and moist air from the remnants of two tropical cyclones over northern Australia, including the category 4 tropical cyclone Trevor. Figure 2.6 highlights a number of ARs that were directly associated with named ex-tropical cyclones. 
(a) Atmospheric River Detected at 1980-03-01-00 UTC

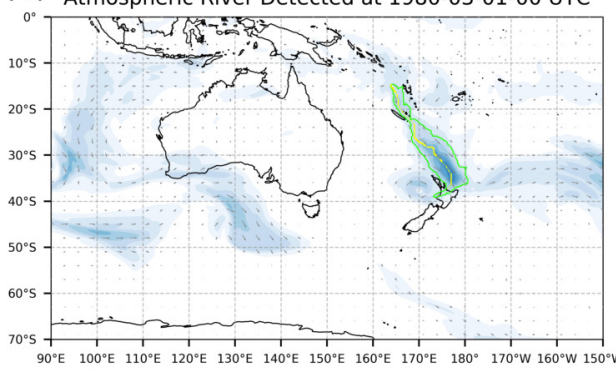

(d) Atmospheric River Detected at 1996-12-29-12 UTC

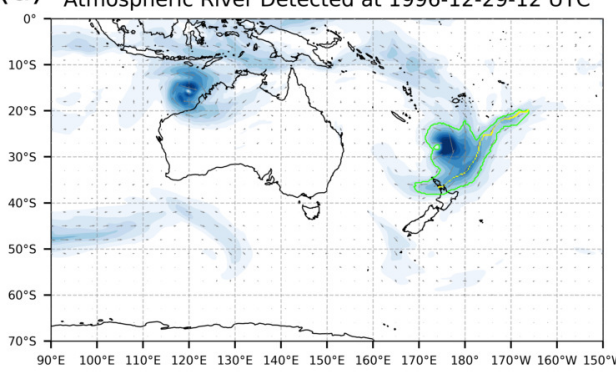

(b) Atmospheric River Detected at 1982-04-09-00 UTC

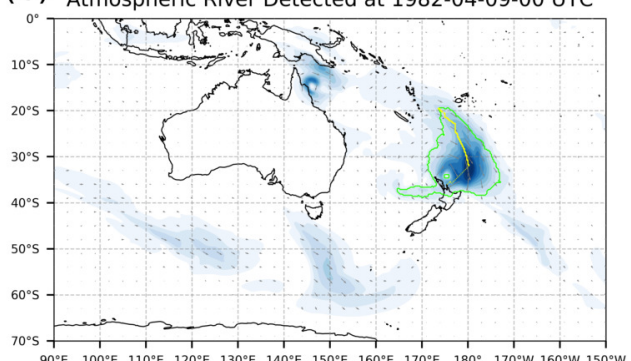

e) Atmospheric River Detected at 1997-03-08-12 UTC

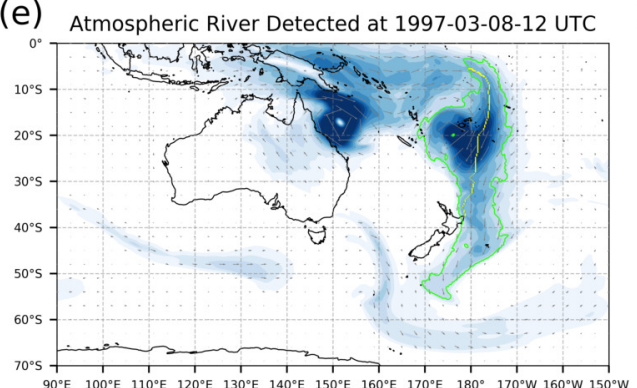

(C) Atmospheric River Detected at 1992-03-06-12 UTC

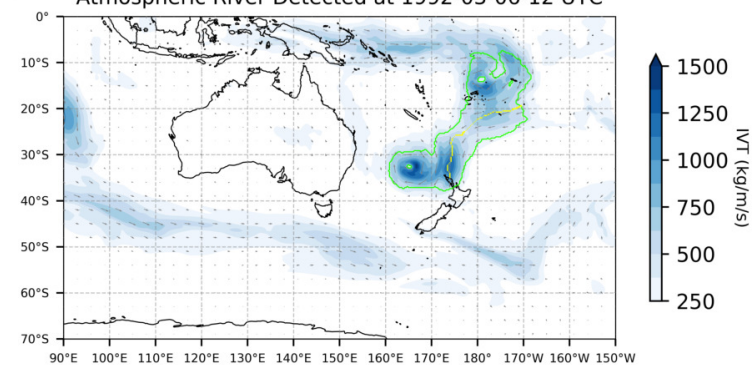

(f) Atmospheric River Detected at 2004-02-27-12 UTC

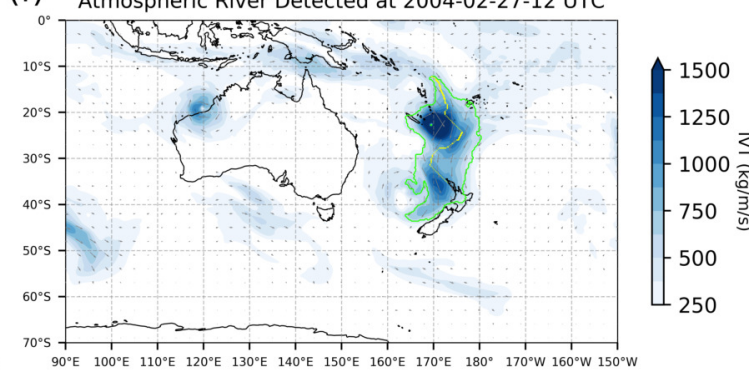

Figure 2.6: A selection of named ex-tropical cyclones that were directly associated with AR landfalls (from the NZ AR dataset). Ex-tropical cyclone (a) Sina, (b) Bernie, (c) Esau, (d) Fergus, (e) Gavin, and (f) Ivy. 
A number of atypical AR geometries are observed involving tropical cyclones, such as multiple tropical cyclones north of New Zealand forming a continuous area of enhanced IVT. Indeed, the interaction of multiple synoptic systems appears to influence the development of a number of North Island ARs, while ARs impacting the western South Island are typically associated with a single lowpressure system south of $40^{\circ}$ latitude. New Zealand is perhaps unique in experiencing such a high number of 'tropical ARs', though ARs associated with tropical cyclones have also been observed in California. Cordeira et al. (2013) investigated two AR events that occurred simultaneously, developing in proximity to three tropical cyclones in the western and central North Pacific. The study found that AR-like structures could form in the absence of an upstream subtropical trough if poleward water vapour flux on the east and poleward side of a tropical cyclone is sufficiently strong (Cordeira et al., 2013). It should be noted that not all ex-tropical cyclones meet the geometry criteria of an AR, thus AR climatologies will exclude a number of these extreme events. Some of New Zealand's most extreme events, e.g. Cyclone Bola, Cyclone Fehi, Cyclone Gita were not associated with ARs. In general, tropical cyclones and ex-tropical cyclones are considered distinct from AR events, though may coincide if the geometry aligns with typical AR structures. Indeed, our detection algorithm is intended to exclude tropical cyclones (with closed low-level atmospheric circulation), but may retain transitioning tropical cyclones and regions of enhanced IVT associated with tropical cyclones. Further research on the interaction between tropical cyclones and ARs, and the moisture sources associated with New Zealand ARs, is required.

The majority of water vapour within ARs is generated through ascent within the warm conveyor belt, rather than long-distance transport of water vapour (Cordeira et al., 2013; Dacre et al., 2015). Dacre et al. (2015) therefore suggests that the term 'atmospheric river', which implies direct and continuous transport of water vapour, is contrary to the notion of ARs as 'footprints left behind by poleward traveling storms'. However, in assessing the dominant moisture sources associated with eastern Pacific ARs, Bao et al. (2006) found a number of ARs that were associated with direct (river-like) poleward transport of tropical moisture. Indeed, a combination of both local (mid-latitude) and remote (tropical) moisture sources appear to contribute to water vapour within New Zealand ARs.

To assess AR differences by location, we define six regional climate zones for New Zealand (based on the regions defined by Mullan (1998)). See Figure 3.3 for a map of the region boundaries. These climate zones are based on an EOF analysis of monthly precipitation anomalies at individual stations, and largely reflect the east-west contrasts induced by the topography and mean westerly flow. The climate zones are the northern North Island (NNI), eastern North Island (ENI), southwestern North Island (SWNI), northern North Island (NSI), eastern South Island (ESI) and western South Island (WSI). These zones have been used in a number of previous studies (e.g. Kidson, 2000; Kidson and Renwick, 2002; Lor- 
rey et al., 2007). We use these climate zones throughout this thesis, to assess the impacts of climate variability on regional AR frequency. While previous investigations have highlighted the fact that New Zealand experiences more ARs during summer (and our analysis supports this fact), the seasonality of AR occurrence varies strongly by region. Figure 2.7 shows the monthly counts of AR landfall in each region, highlighting regional differences in the seasonal cycle. For the North Island, we find a clear peak in AR frequency in June, while South Island AR frequency peaks in October. Interestingly, all regions exhibit a monthly minimum in AR occurrence during April.

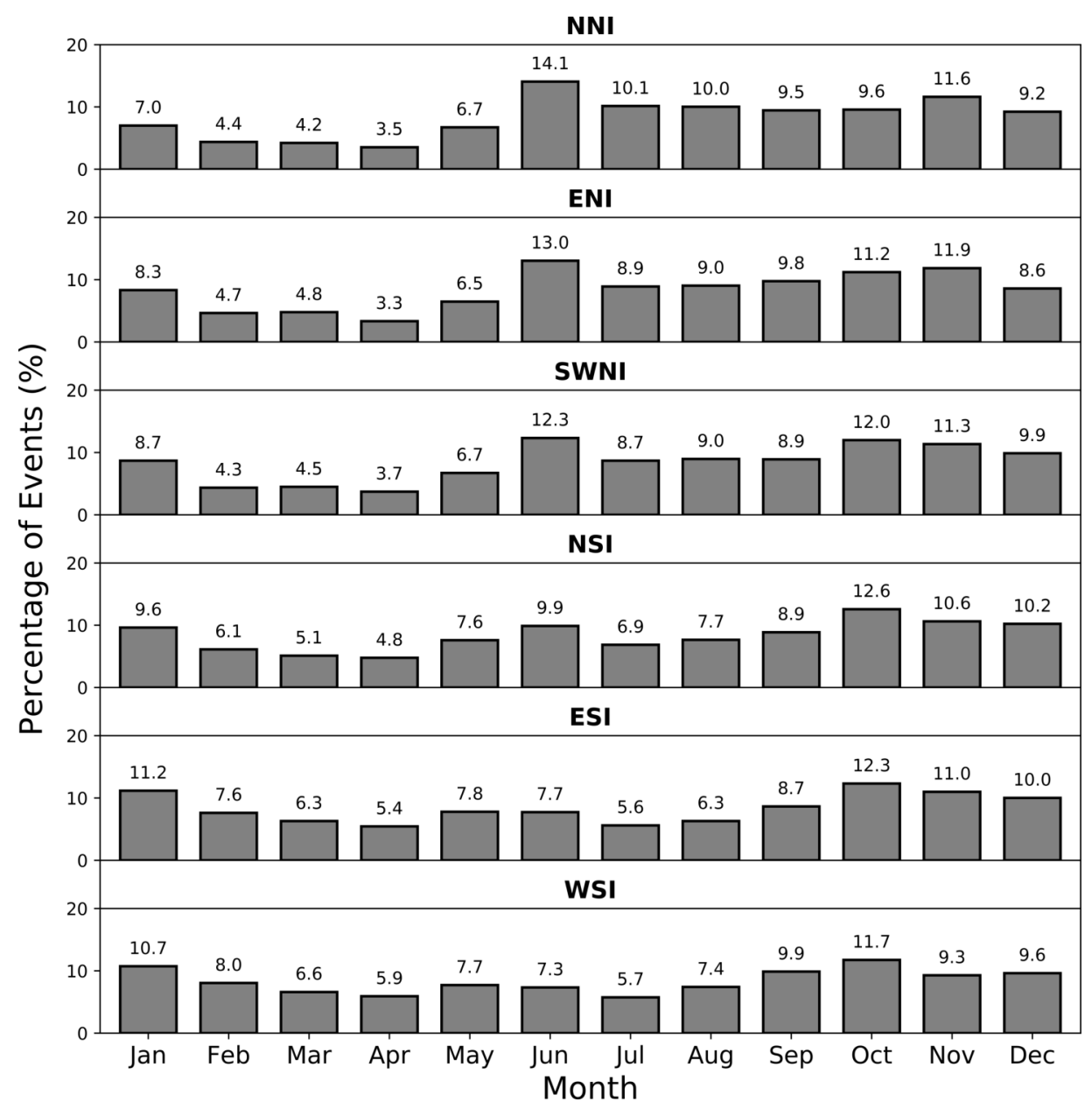

Figure 2.7: Monthly frequency of landfalling ARs by region. Derived from the NZ AR dataset.

Figure 2.8 shows the seasonal variability (coefficient of variation) in AR occurrence. For the North Island, the season of largest variation in year-to-year AR fre- 
quency is during Summer and Autumn. In particular, we see that AR frequency north of the mid-latitude storm track $\left(30^{\circ} \mathrm{S}\right)$ displays significant year-round variability. Thus, ARs of tropical origin making landfall in New Zealand are likely dependent on tropical sea-surface temperatures, and modes of tropical variability (particularly during summer). We explore the impacts of tropical Pacific forcing on AR frequency in Chapter 4. For the South Island, winter months have a higher variability in AR frequency, when the storm track is located further south. There is little variability in summer AR frequency in the southwestern South Island, where AR landfall frequency is consistently high.

To summarize this section, ARs make landfall all along the New Zealand coastline, but most frequently in the western South Island (14-16 AR days per year), with an absolute overland maximum over Fiordland ( $>17$ AR days per year). There is a notable seasonality in AR occurrence, which varies significantly by region. The occurrence of ARs reaches a maximum in the South Island during summer, while AR frequency over the northern North Island peaks during winter.

\subsection{AR Characteristics}

Rosier et al. (2015) found that the combination of a synoptic situation favouring northeasterly flow over northern New Zealand, and extremely moist air of subtropical origin, contributed to extreme winter rainfall totals in Northland. Rosier et al. (2015) speculated that such events would be identified as ARs. Indeed, while the mean IVT direction of most ARs making landfall in New Zealand is northwesterly, a number of northeasterly ARs are observed making landfall on the east coast of the country (Figure 2.9). In particular, we find that $17 \%$ of ARs have an easterly IVT direction at landfall, and 3.8\% of ARs have an easterly mean IVT direction. Of the ARs with an easterly mean IVT direction, 70\% made landfall in the northern North Island. Figure 2.9 shows histograms of AR characteristics, from the NZ AR dataset. ARs making landfall in New Zealand have a mean length of $3146 \mathrm{~km}$ and a mean length/width ratio of 6.7. Mean IVT magnitude over the AR region ranges between $362-993 \mathrm{kgm}^{-1} \mathrm{~s}^{-1}$, with a mean of 616 $\mathrm{kgm}^{-1} \mathrm{~s}^{-1}$. IVT magnitude at landfall ranges between $252-2100 \mathrm{kgm}^{-1} \mathrm{~s}^{-1}$, with a mean of $729 \mathrm{kgm}^{-1} \mathrm{~s}^{-1}$.

Prince et al. (2021) used a five-category AR rank scale to characterize the intensity of AR events in New Zealand, derived from the global AR dataset of Guan \& Waliser (2015). The scale is based on the IVT magnitude and duration of AR conditions over each location (Ralph et al., 2019). Throughout this thesis, we do not adopt a scale to quantify AR intensity (aside from mean IVT), but instead classify 


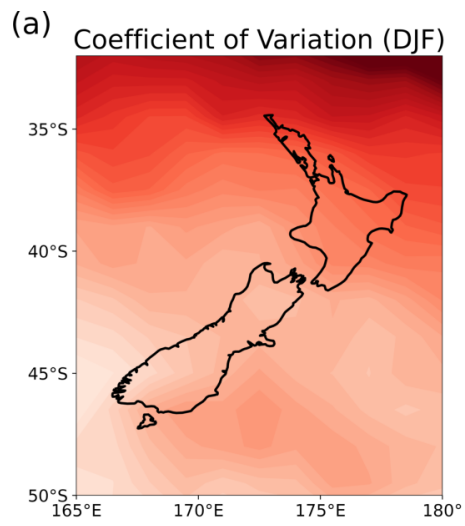

(c) Coefficient of Variation (MAM)

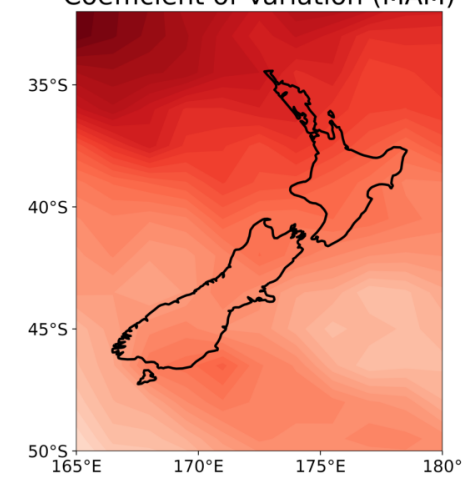

(e)

e) Coefficient of Variation (JJA)

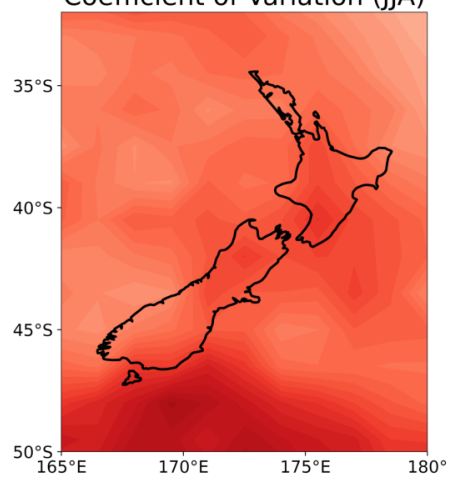

(g) Coefficient of Variation (SON)

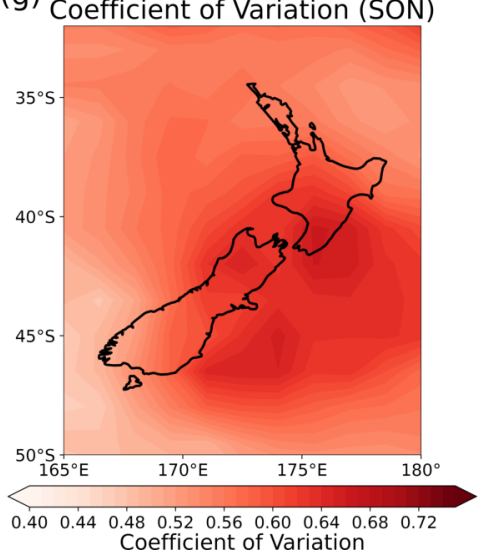

(b) Coefficient of Variation (DJF)

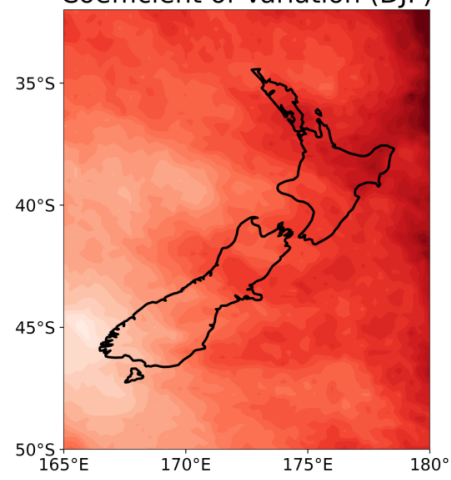

(d) Coefficient of Variation (MAM)

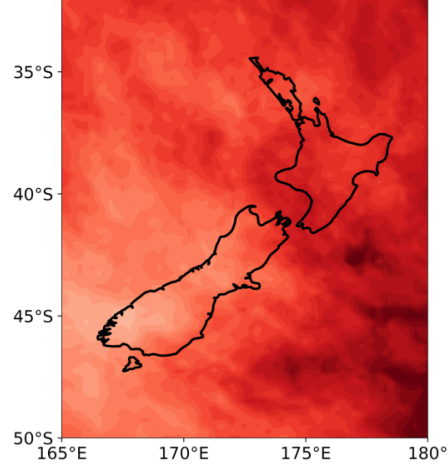

(f)

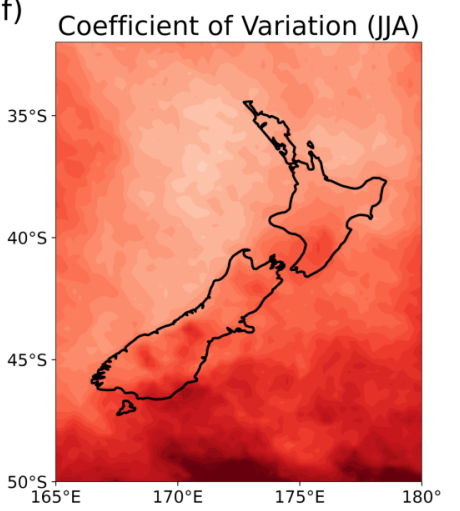

(h) Coefficient of Variation (SON)

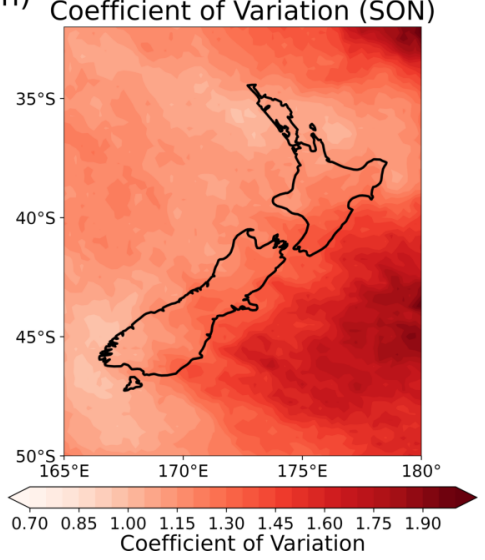

Figure 2.8: Coefficient of variation of monthly AR frequency for each season. (a, c, e, g) are derived from the global AR dataset (Guan \& Waliser, 2015), while (b, d, f, h) are derived from the NZ AR dataset. 

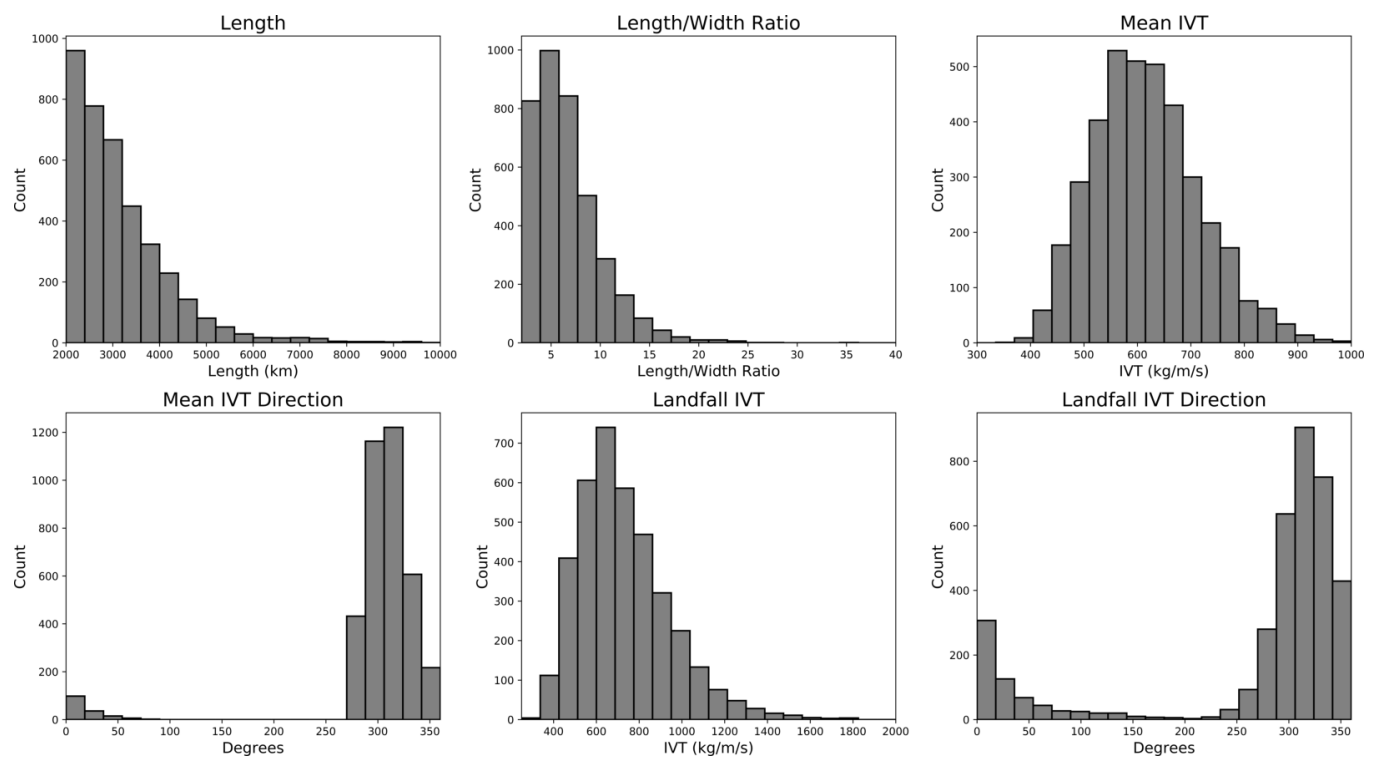

Figure 2.9: Histograms of AR characteristics for all landfalling ARs detected over the 1979-2020 period. Derived from the NZ AR dataset.

ARs in terms of their observed impacts (e.g. An AR is classified as 'Extreme' if a 24 hour rainfall total above $100 \mathrm{~mm}$ is observed). We will assess the impacts of ARs on New Zealand precipitation in the next chapter.

Figure 2.10 shows the mean duration, mean IVT magnitude, and mean IVT direction under AR conditions for each grid-cell (derived from the NZ AR dataset). The mean duration of AR conditions is highest for the northern and southwestern North Island, persisting for more than 11 hours on average. The strongest ARs to the northeast of New Zealand are associated with enhanced moisture associated with tropical systems which can persist for a significant duration. ARs impacting southern New Zealand are usually associated with faster moving systems, with a more zonal mean IVT direction. The mean IVT under AR conditions sharply decreases on the leeward side of the Southern Alps and Central Plateau, due to orographic rainout. In the southwestern South Island, strong westerly winds drive very strong IVT magnitudes, however AR events in this region are often shortlived. Indeed, 'Weak ARs' (max IVT less that $500 \mathrm{kgm}^{-1} \mathrm{~s}^{-1}$ and duration less than 24 hours) and Category 1 ARs are most common in the southwestern South Island, and to the south of New Zealand (Prince et al., 2021). The mean IVT direction at landfall for all ARs is $328^{\circ}$ (north-northwest), though for the coastal southwestern South Island the formation of a barrier jet forces a more northerly IVT direction at landfall. For northern regions, the IVT direction at landfall may be directly from the north, or even the northeast, associated with lower latitude cyclonic systems. There is also a slight seasonal change in the IVT direction at 
landfall associated with ARs; the mean IVT direction over the country shifts to a more northerly direction during winter.

(a) Frequency

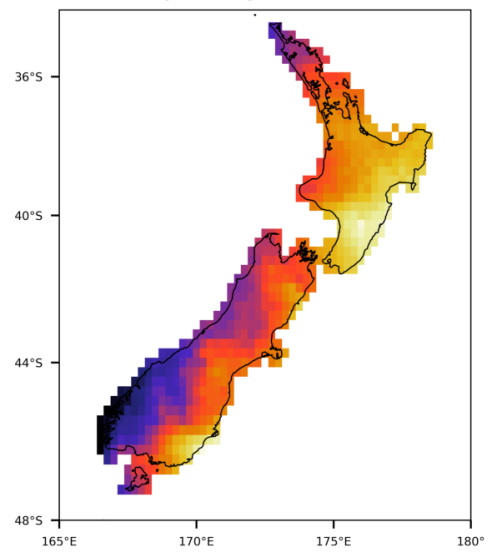

(c) Mean IVT Magnitude

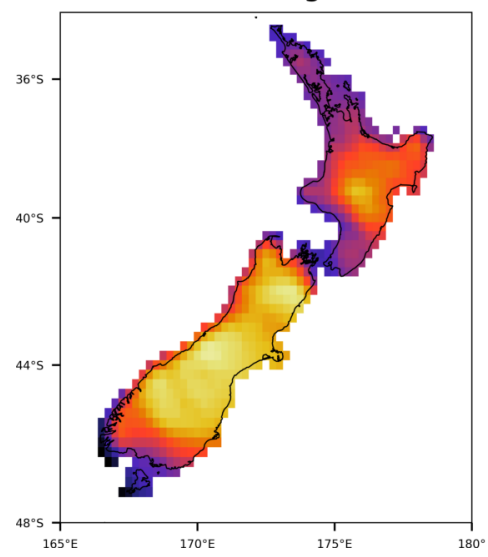

(b) Duration

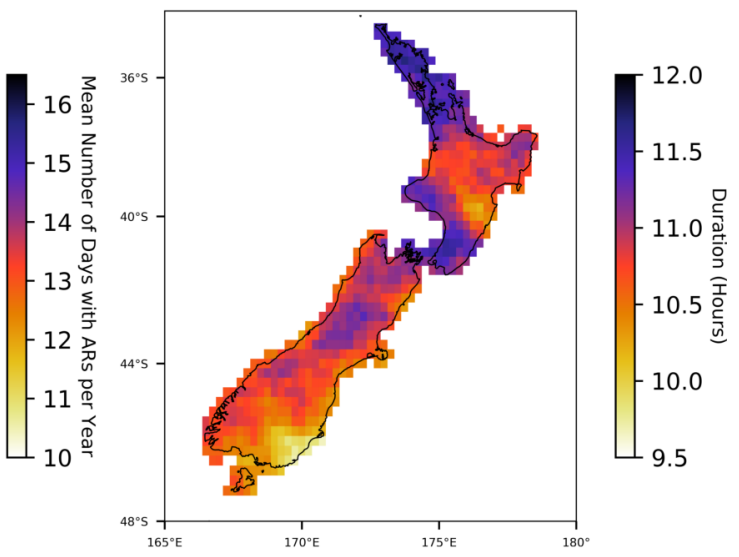

(d) Mean IVT Direction

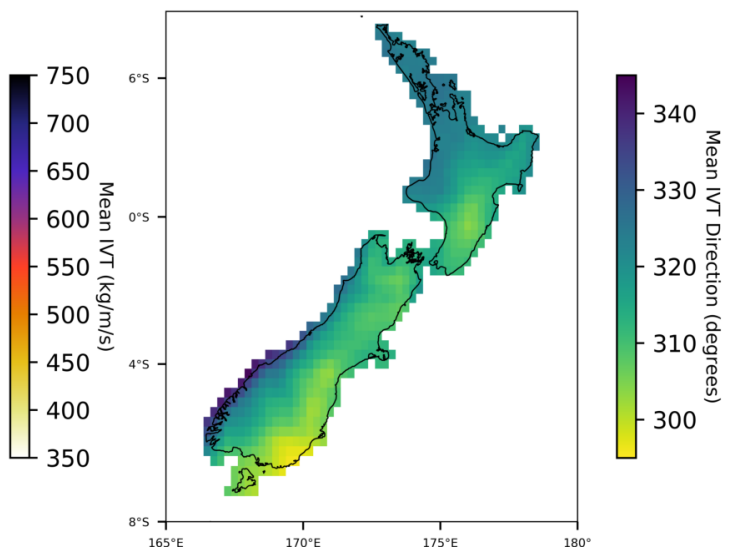

Figure 2.10: Mean AR condition characteristics. For each grid cell, mean values are computed for all ARs detected over the 1979-2020 period. (a) Frequency of AR conditions, (b) Mean duration of AR conditions, (c) Mean IVT during AR conditions, (d) Mean IVT direction during AR conditions. Derived from the NZ AR dataset.

An extensive variety of AR geometries are observed in New Zealand, exhibiting a range of intensities, orientations, and genesis locations. In order to better understand the range of observed AR geometries, we further categorize ARs in terms of the synoptic patterns that drive them. 


\subsection{Synoptic Types}

Synoptic climatology refers to the study of climate from the perspective of synopticscale atmospheric circulation patterns. In this field, a common methodology is to group similar weather patterns into distinct categories. This has the advantage of scaling down the wide variety of observed weather patterns into a small number of types, allowing us to statistically assess connections between circulation patterns and other climate features. In our case, we are interested in the different circulation patterns associated with landfalling ARs in New Zealand. There are currently no investigations of AR occurrence and synoptic types in New Zealand. Indeed, formal investigations of ARs and synoptic types in other regions of the world are few in number.

The synoptic type classification method is well established and was originally used to improve regional forecasting techniques (Barry, 2005). A wide variety of synoptic typing methods have been employed in different regions of the world, typically based on daily surface or upper-level pressure fields. We make use of an existing scheme, the Kidson Type (KT) (Kidson, 2000). This classification scheme allows us to characterize AR landfalls in terms of general weather patterns. Thus, we build on previous analyses of ARs by investigating relationships between ARs and Kidson weather types (which are known to be influenced by large-scale climate variability like ENSO and SAM), to better understand the synoptic conditions that caused each AR event.

'Kidson types' are a set of 12 synoptic types for the New Zealand region defined by Kidson (2000) (updated 12-hourly Kidson type data was provided by Prof. James Renwick). The Kidson type classification is based on a cluster analysis using $1000 \mathrm{hPa}$ geopotential height fields from gridded atmospheric reanalysis (NCEP/NCAR reanalysis version 1). Other synoptic types have been defined for the New Zealand region. For example, Jiang (2011) used a different method from Kidson (2000) based on rotated T-mode principal component analysis and convergent K-means clustering, identifying 12 synoptic types for New Zealand (similar to those identified by Kidson (2000)). Kidson types have been employed in a number of previous studies, including investigations of snowfall and glacier mass balance (Purdie et al., 2011; Webster et al., 2015; Cullen et al., 2019), hydrology and drought (McKerchar et al., 2010; Salinger and Porteous, 2014), and paleoclimatology (Lorrey et al., 2007, 2014). Griffiths (2011) investigated the relationship between each Kidson type and extreme daily rainfalls ('Rx1day') from 22 rainfall station rainfall records across New Zealand on monthly, seasonal and annual time scales. Significant relationships between seasonal station rainfall extremes and the SAM, ENSO and IOD were also identified. Renwick (2011) further explored relationships between Kidson types and SAM/ENSO and investigated the mean magnitude of temperatures and rainfall associated with each Kidson type. Kidson types have the potential to be used in experimental forecasts of ARs 
and extreme rainfall in New Zealand, and are therefore worth examining. Specifically, Kidson types may be useful as a proxy to help identify and forecast the most damaging ARs and extreme rainfall events.

The mean $1000 \mathrm{hPa}$ height fields for each Kidson type are shown in 2.11.
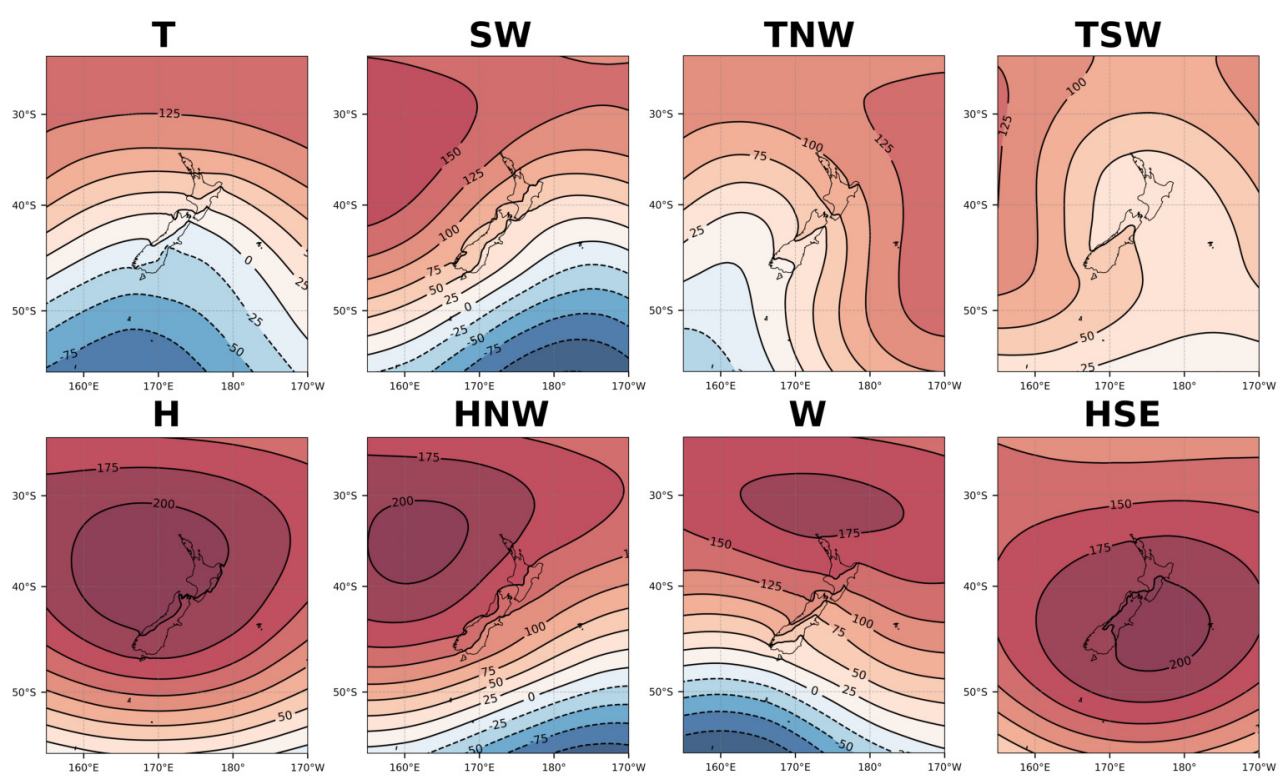

HNW

$\mathbf{W}$

HSE
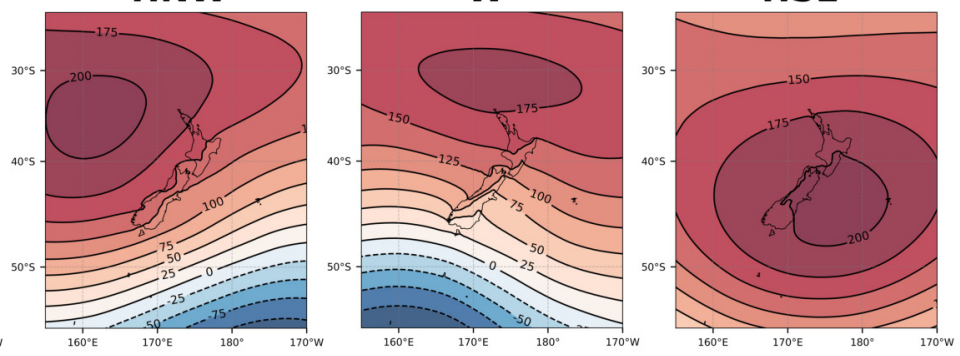

HE

NE

HW

$\mathbf{R}$

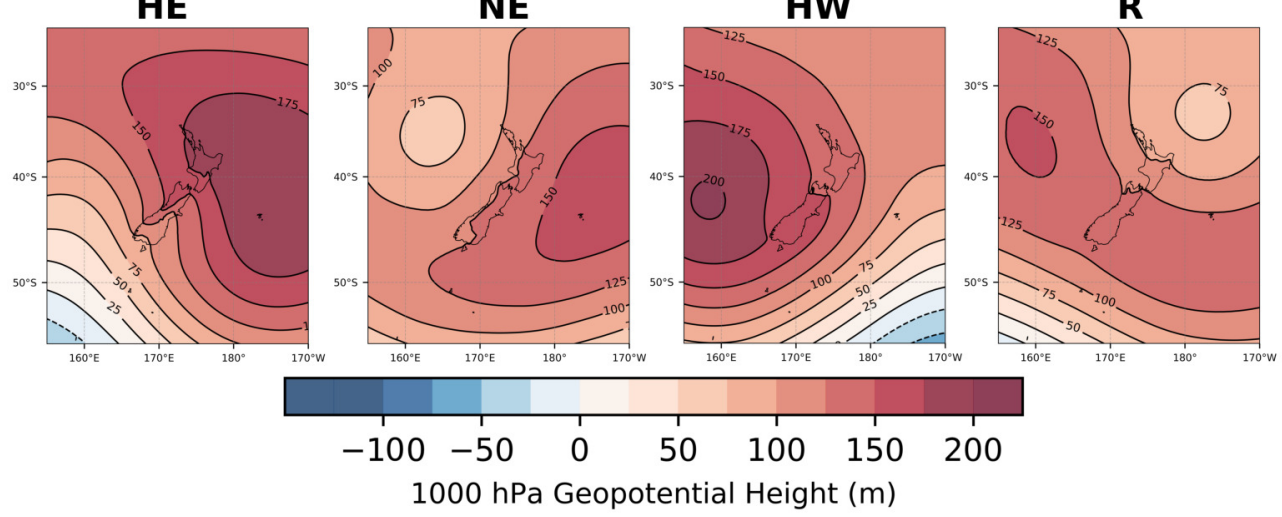

Figure 2.11: The 12 Kidson synoptic types represented by mean $1000 \mathrm{hPa}$ geopotential height composites using ERA5 data.

Each Kidson type can be further classed as belonging to one of three regimes:

Trough: Frequent troughs crossing the country (types T, SW, TNW, TSW).

Zonal: Highs to the north with strong zonal flow to the south of New Zealand (types $\mathrm{H}, \mathrm{HNW}, \mathrm{W}$ ). 


\begin{tabular}{cccccc}
\hline Kidson Type & Annual & MAM & JJA & SON & DJF \\
\hline T & 11.6 & 7.7 & 11.9 & 15.8 & 11.0 \\
SW & 12.0 & 10.4 & 11.6 & 15.8 & 10.1 \\
TNW & 7.0 & 4.9 & 7.4 & 7.0 & 8.9 \\
TSW & 5.69 & 3.9 & 6.7 & 5.1 & 7.0 \\
H & 13.8 & 17.7 & 16.4 & 13.4 & 7.7 \\
HNW & 7.8 & 8.3 & 9.4 & 8.7 & 4.7 \\
W & 5.6 & 4.4 & 5.9 & 8.1 & 3.8 \\
HSE & 13.5 & 18.8 & 10.2 & 8.2 & 16.9 \\
HE & 7.6 & 8.3 & 8.6 & 7.3 & 6.4 \\
NE & 5.4 & 5.3 & 4.0 & 3.8 & 8.6 \\
HW & 5.4 & 6.0 & 4.2 & 3.6 & 7.8 \\
R & 4.6 & 4.5 & 3.7 & 3.0 & 7.0 \\
\hline
\end{tabular}

Table 2.1: Annual and seasonal Kidson type frequency (\% occurrence) for the Mar-1979 to Feb-2020 period.

Blocking: Blocking patterns with highs more prominent in the south (types HSE, HE, NE, HW, R).

The frequency of each Kidson type varies by season. Figure 2.1 shows the relative frequencies of each Kidson type over the 1979-2020 period. In general, the blocking regime is most frequent in summer and autumn, the zonal regime is less common in summer, and the trough regime is less frequent in autumn (Kidson, 2000). Kidson (2000), Griffiths (2011), and Renwick (2011) also show that the relative frequencies of each type are modulated by the SAM, ENSO and the IOD. Using the KT framework we may associate each AR with a weather circulation pattern. This allows us to explore relationships between synoptic conditions and ARs, and examine the synoptic conditions leading to the most extreme AR events. In this chapter we primarily use the NZ detection algorithm (Kennett, 2020) as it captures the most extreme events and more accurately represents the AR landfall location. As Kidson types are defined 12-hourly, while ARs are detected 6-hourly, if an AR falls between Kidson time-steps it is associated with the Kidson types of the two adjacent time-steps. Thus some ARs may be associated with more than one Kidson type.

We classify the most intense ARs in terms of daily precipitation (see Chapter 3 for methods). If at least one station records a daily rainfall total above the 99th percentile (defined relative to all daily rainfall observations for that station), either the day of or day after AR landfall, that AR is labeled as a 'Heavy' AR. If at least one station records a daily rainfall total above $100 \mathrm{~mm}$, either the day of or day after AR landfall, that AR is labeled as an 'Extreme' AR. Again, to assess 
regional impacts, we use the six regional climate zones defined by Mullan (1998). An AR must overlap a climate zone to be counted as landfalling for that zone. Similarly, the daily station records from within each climate zone define whether an AR is a 'Heavy' or 'Extreme' event for that zone.

\subsubsection{Synoptic AR Climatology}

In general, the passage of troughs across New Zealand is characterized by the TNW, T/TSW, SW sequence of Kidson types (Kidson, 2000) and the majority of ARs are associated with these 'trough' regime Kidson types, though not exclusively. Figure 2.12 shows plots of AR frequency in the southwest Pacific region, concurrent with each Kidson type. AR events in New Zealand have much more complex geometries compared with other regions of high AR landfall. Unlike the Pacific coast of North America, Western Europe and the Chilean Andes, which feature an extended western coastline, New Zealand is located in open ocean, exposed to both the mid-latitude storm track and tropical moisture sources to the north. Figure 2.13 shows detected AR axes for each Kidson type, displaying a wide range of orientations. The majority of ARs making landfall in New Zealand were associated with the T (19.9\%), TNW (14.5\%), HE (10.5\%), and W $(9.9 \%)$ types. In particular, for all regions except the western South Island, the majority of AR landfalls occurred during the T synoptic type. For the western South Island, AR landfalls were recorded most frequently for HE $(16.0 \%)$, TNW $(16.0 \%), \mathrm{T}(15.7 \%)$, and $\mathrm{W}(14.6 \%)$ types. Thus HE and TNW types, which are associated with northwesterly advection of moisture, drive AR landfalls in the western South Island. In general, the increased monthly frequency of 'blocking' regime types was correlated with a decrease in AR frequency overall, while an increase in the frequency of the 'zonal' and 'trough' regimes was associated with an increase in AR frequency. Figure 2.13 also highlights the spatial extent of ARs making landfall in New Zealand, with a number of ARs extending thousands of kilometres to the South Australian Basin, northward to the tropical western pacific $20^{\circ} \mathrm{S}$, and even extending to the Java sea and eastern Indian Ocean. 


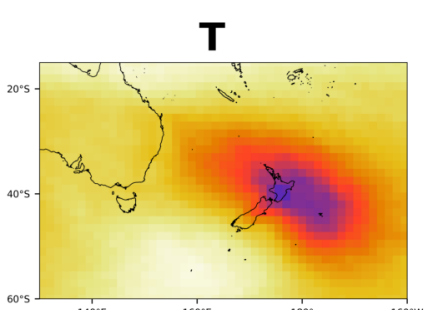

H

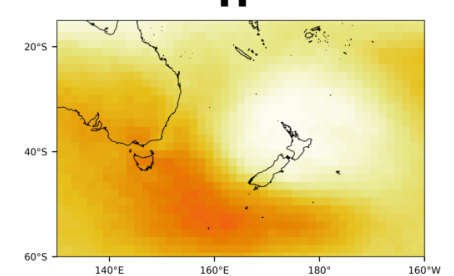

HE
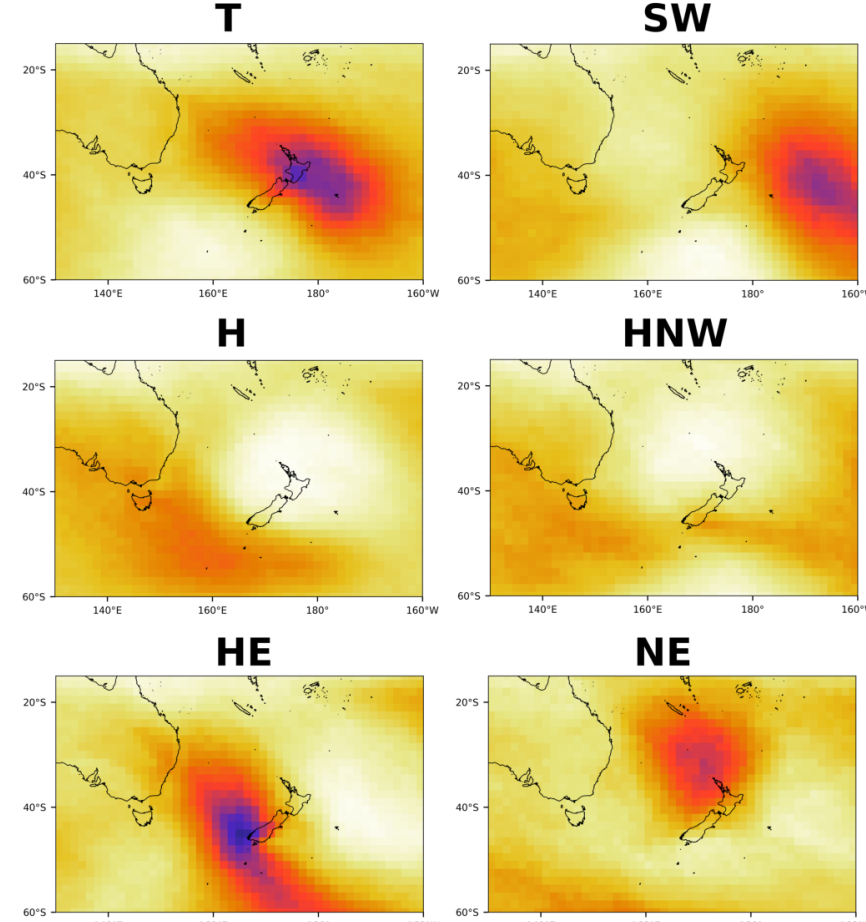

HNW

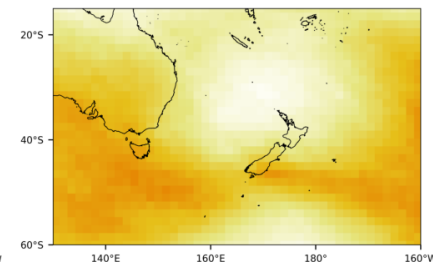

NE

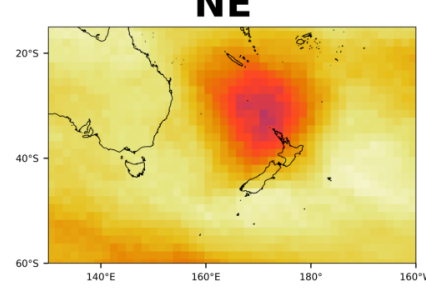

TNW
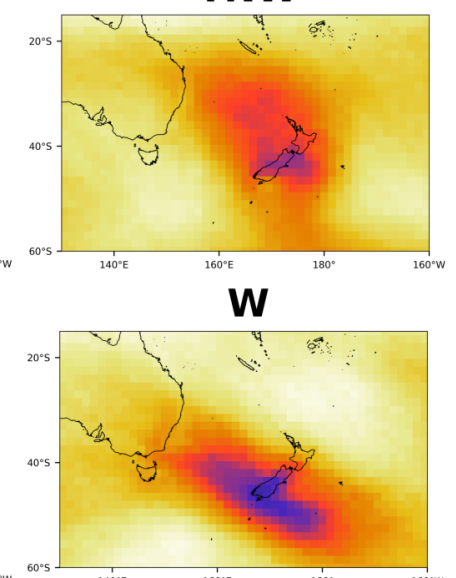

HW

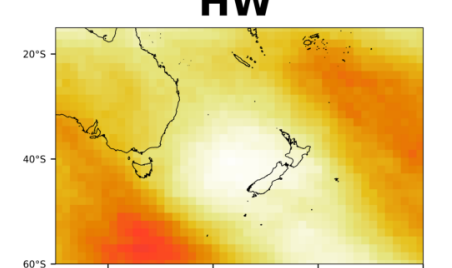

TSW

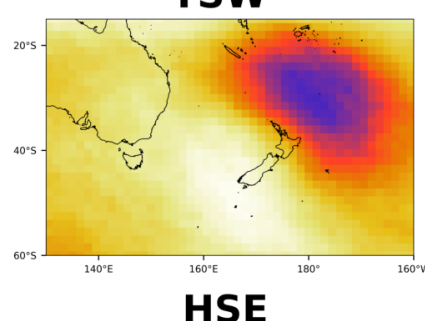

HSE

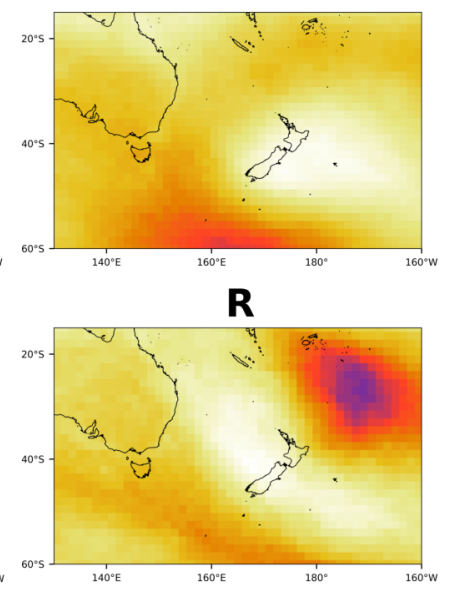

N

节

อิ

त्ञ

Figure 2.12: AR frequency for each Kidson type, derived from the global AR dataset of Guan \& Waliser (2015). 


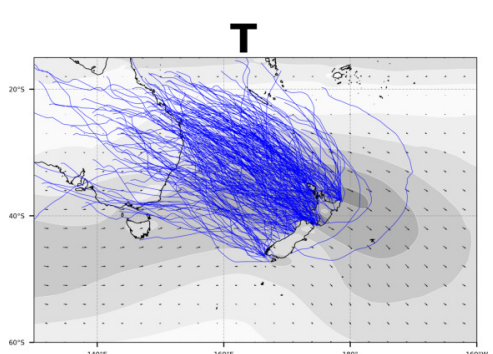

H

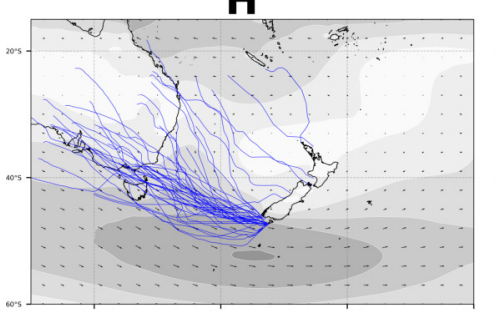

HE

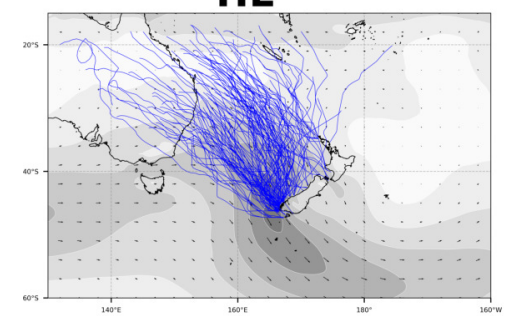

SW

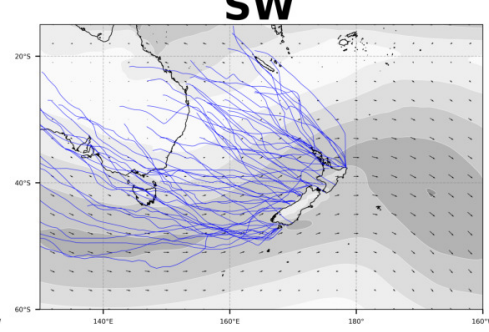

HNW

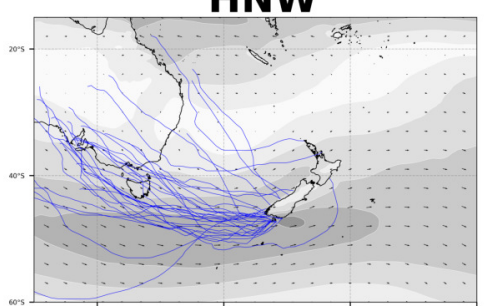

NE

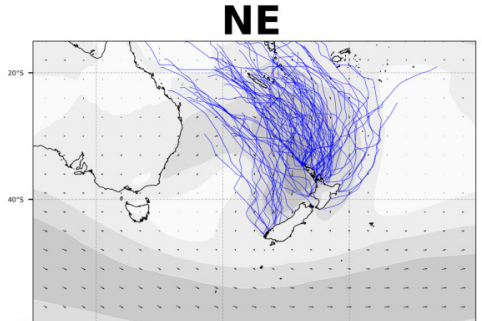

(100

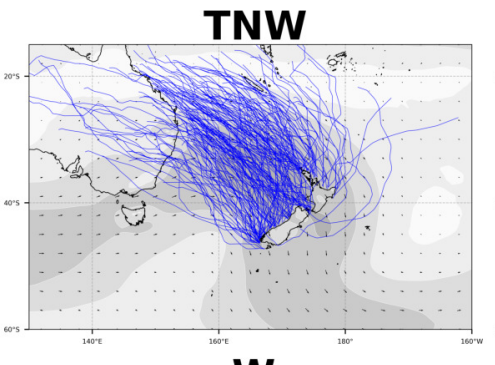

w

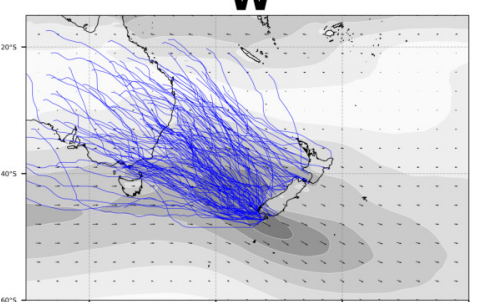

HW

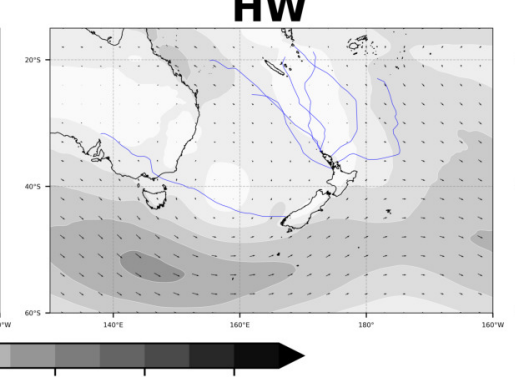

400

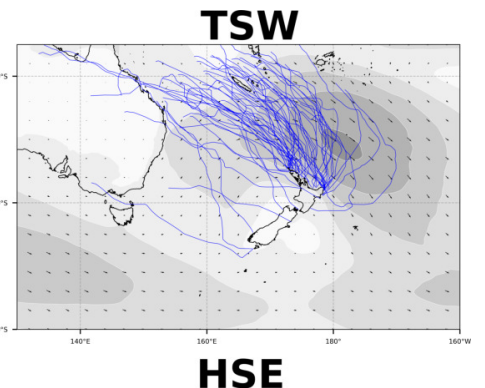

HSE

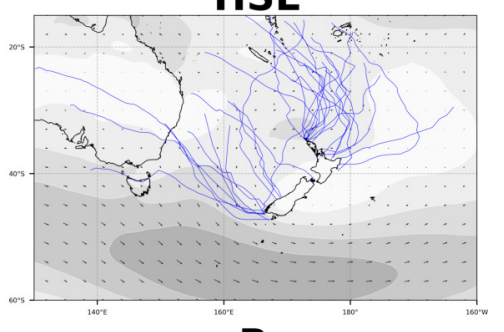

$\mathbf{R}$

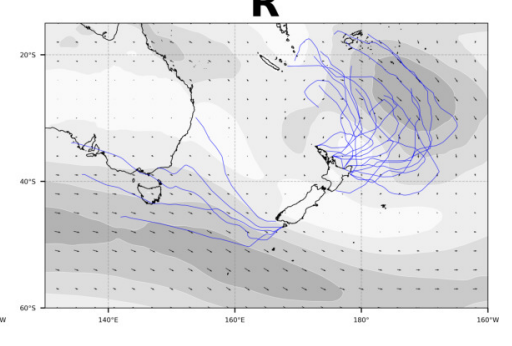

Figure 2.13: Kidson type composites of mean IVT (shaded contours and vectors) and AR axes.
की

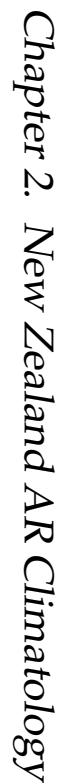


Unlike other regions of the world, where ARs are typically driven by similar and consistent synoptic-scale patterns, a wide range of circulation patterns contribute to ARs in New Zealand. Further, there are significant differences in the primary synoptic drivers of AR landfalls for each region. Figure 2.2 shows the seasonal fraction of regional AR landfalls associated with each Kidson type. The primary synoptic patterns associated with ARs for each region did not display significant seasonal variation. However, some slight differences between summer and winter were observed. For the North Island, a larger fraction of T ARs are observed during spring and summer, and a larger fraction of W, HSE, HE, and NE ARs are observed during autumn and winter. For the South Island, a larger fraction of $\mathrm{T}$ and SW ARs are observed during spring and summer, and a larger fraction of $\mathrm{H}$, HSE, HE and NE ARs are observed during autumn and winter.

Table 2.2: Percentage of landfalling ARs associated with each Kidson type. Calculated for each region between 1979-2019. Cells are shaded proportional to their values.

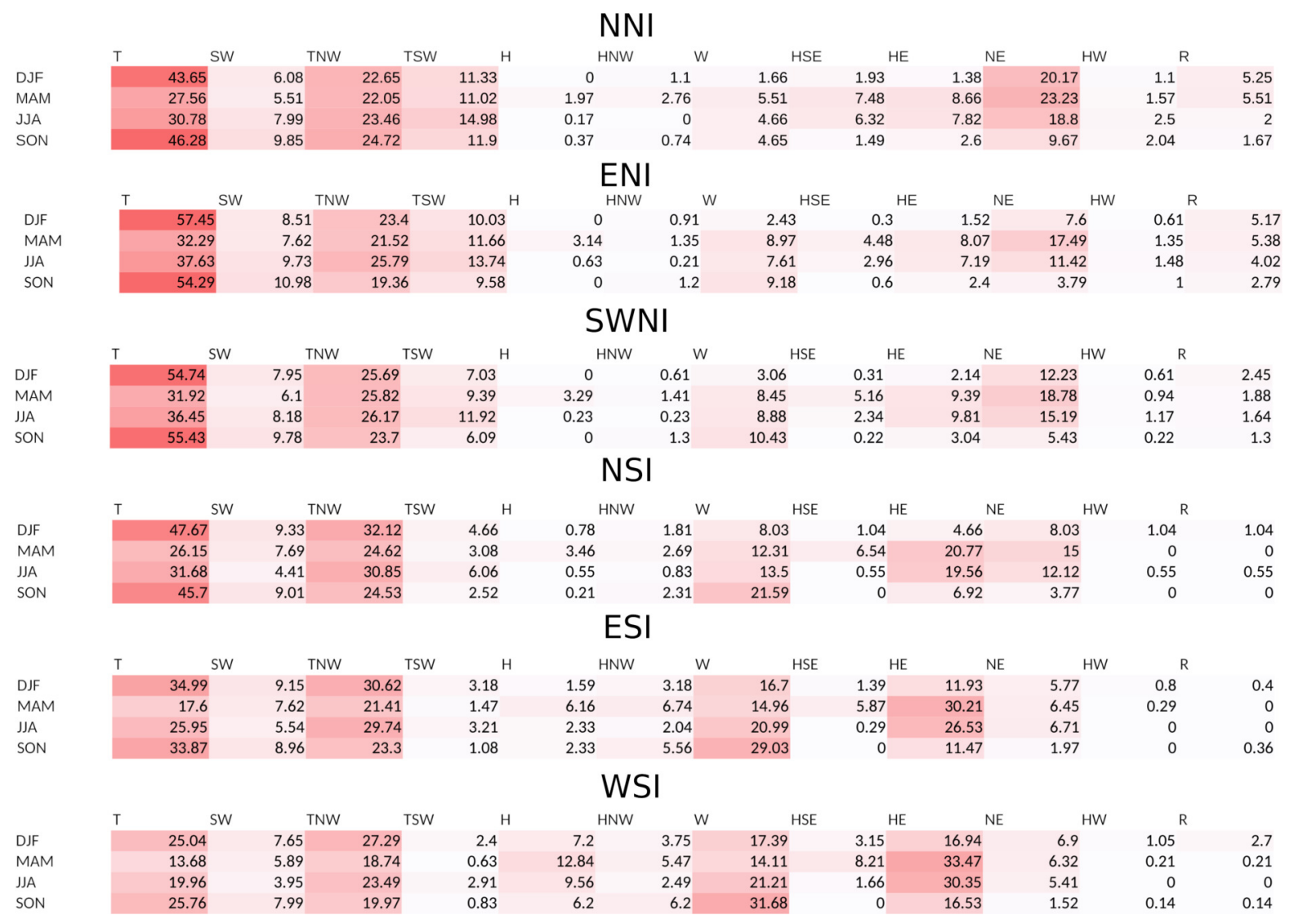

Overall, seasonal differences in the favoured synoptic conditions are small. These 
results suggest that the synoptic drivers of ARs do not vary significantly between summer and winter. Rather, seasonal variation of AR occurrence is related to changes in the frequency of the synoptic patterns themselves.

We also assess regional Kidson types frequencies associated with 'heavy' and 'extreme' ARs. For the northern North Island, heavy and extreme AR events are favoured by northeasterly flow over the region. For the northern North Island, $54 \%$ of summer extreme ARs were associated with the NE Kidson type, compared to $20 \%$ of all summer NNI ARs. This is in agreement with the findings of Rosier et al. (2015) who found that the combination of a synoptic situation favouring northeasterly flow over northern New Zealand, and extremely moist air of subtropical origin, contributed to extreme winter rainfall totals in Northland in 2014. ARs associated with TSW and NE types (with enhanced IVT to the northeast of New Zealand) are most likely to contribute to daily rainfall totals above the 99th percentile ( $94 \%$ of AR events associated with these types). For the eastern North Island, extreme events are favoured by both NE and R Kidson types. For the North Island and northern South Island, trough Kidson types (T, SW and TNW) are less favourable for extreme AR events. However, heavy and extreme ARs in the western and eastern South Island consist of a higher fraction of T, TNW and TSW Kidson types. We also investigate the relationship between Kidson type and AR characteristics (such as IVT intensity and duration). As expected, Kidson types associated with strong zonal flow (T, SW, TNW, W) are associated with higher IVT magnitude at landfall.

The intensity and position of ARs is strongly modulated by the relative positions of the associated low and high pressure centres (Zhang et al., 2019). In particular, the characteristics and structure of New Zealand ARs is likely significantly influenced by the preceding ridge environment. A blocking high is a persistent high-pressure system that remains almost stationary for more than 24 hours, strongly influencing the regional circulation. While blocking highs in New Zealand are typically associated with mostly dry weather and even drought, they can assist in drawing down warm, moist air from the tropics. Deflection of cyclones from their usual paths can induce extreme weather conditions, especially equatorward of the blocking high (Taljaard, 1972). In New Zealand, a rapid succession of cyclones may occasionally follow a blocking event, which can pose potential for flooding (Dravitzki and McGregor, 2011). Reid et al. (2021) found that ARs contributing to the highest daily rainfall totals in New Zealand were associated with higher maximum IVT (statistically significant) and were of longer average duration. Thus, blocking events in the New Zealand sector may be precursors of extreme AR events. Indeed, a number of identified extreme AR events in New Zealand were associated with notable blocking highs. Although blocking highs are recognized as influencing the intensity of heavy rainfall events (e.g. Rabinowitz et al., 2018), there have been few formal studies of how atmospheric blocking influences AR activity. While investigating synoptic-scale precursors of 
AR landfalls in northwestern North America, Benedict et al. (2019) found that high-latitude blocking anticyclones were associated with increased probability of AR occurrence at lead times of 1-2 weeks. In New Zealand, we also expect that blocking events strongly influence the intensity of AR events, particularly in summer, as well as extending the duration of AR events. We therefore suggest this as an avenue of future research.

\subsection{Conclusions}

We develop and implement an automated AR detection algorithm for New Zealand over the 1979-2019 period. Compared to past studies of ARs in New Zealand, fewer ARs overall were captured by our detection algorithm, though previously identified patterns are maintained. Most importantly, our algorithm is able to successfully capture the most extreme events (i.e. events with significant flooding potential). Using our detection algorithm, we find a significant number of ARs making landfall in the North Island. Notably, we find that AR conditions over the northern North Island are detected for $46 \%$ of all landfalling ARs. These results suggest that previous investigations of New Zealand ARs, using the global detection algorithm of Guan \& Waliser (2015), may underestimate the number of AR landfalls (and impacts) in the North Island (particularly the Far North). As this is likely due to the geometry constraints, we suggest use of AR detection algorithms employing both higher spatial resolution and higher IVT threshold for future New Zealand studies.

While ARs occur throughout the year, there exists a clear seasonality in the spatial distribution of AR frequency. Thus, any investigation related to the modulation of AR activity must be understood in terms of this seasonal cycle. We find that ARs make landfall most frequently in the western South Island during late spring and early summer (SONDJF), and in the North Island during winter and spring (JJASON). This seasonality is mainly linked to displacement of the storm track to the north and south of New Zealand during winter, related to seasonal changes in the mean position of the subtropical and polar jet streams (i.e. the wintertime split-jet), with some contribution from a general increase in atmospheric moisture availability during the warmer months.

We also investigated relationships between ARs and Kidson weather types, to better understand the synoptic conditions that caused each AR event. We found significant regional differences in the synoptic-scale drivers of AR landfalls. ARs can roughly be characterized into two types; those making landfall in the South Island driven by $\mathrm{H}, \mathrm{HNW}, \mathrm{W}, \mathrm{SW}$, and HE Kidson types (associated with northwesterly airflow and enhanced IVT to the west of the country), and those making landfall in the northern North Island driven by TSW, NE, HW, and R Kidson types (associated with low-latitude troughs/subtropical systems and enhanced 
IVT to the northeast of the country). The results in this chapter give context to the remainder of the thesis, where we discuss the impacts and drivers of ARs. 


\section{Chapter 3}

\section{Impact of ARs on Precipitation}

In March 2019, an extreme AR event led to significant flooding on the West Coast, which culminated in the collapse of the Waiho Bridge ${ }^{1}$. A state of emergency was declared in Westland as extensive flooding led to evacuations, power outages and the closure of major roads, leaving the Westland community isolated. At least one person died during the event after being swept away by the floodwaters (Guildford, 2019). The Waiho River bridge connecting Franz Josef and Fox Glacier was destroyed by the floodwaters, and the Haast River recorded its second highest water level since 1969 (NIWA Monthly Climate Summary, March 2019). The event had an estimated repair cost of $\$ 9.5 \mathrm{~m}$, and cost the West Coast economy in excess of $\$ 1 \mathrm{~m}$ a day while the bridge was inactive (approximately $\$ 48 \mathrm{~m}$ in total) (Guildford, 2019). A 48-hour precipitation record for New Zealand was recorded at Cropp River, with $1086 \mathrm{~mm}$ falling between the 25-26th of March (equivalent to the mean annual rainfall of Auckland). This surpassed the previous record of $1049 \mathrm{~mm}$, which was also recorded at Cropp River between 12-13 December 1995 (NIWA Monthly Climate Summary, March 2019).

Figure 3.2 shows the synoptic situation for the event. On the 24th of March, an atmospheric river began to form over the Tasman, sourcing warm and moist air from the remnants of two tropical cyclones over northern Australia (including tropical cyclone Trevor, category 4). The AR formed ahead of a system of fronts, driven by a developed low-pressure system to the southwest of New Zealand. Off the east coast of New Zealand, a large area of high pressure (a blocking high) persisted, concentrating northwesterly flow toward the South Island and extending the duration of the event. The event was associated with the HE Kidson type (Kidson, 2000), transitioning into the W Kidson type as the front moved eastward. On the 25th of March, the AR made landfall, beginning a continuous period of strong moisture flux for the South Island lasting 48 hours. The pro-

\footnotetext{
${ }^{1}$ This event was one of the primary catalysts for this thesis.
} 


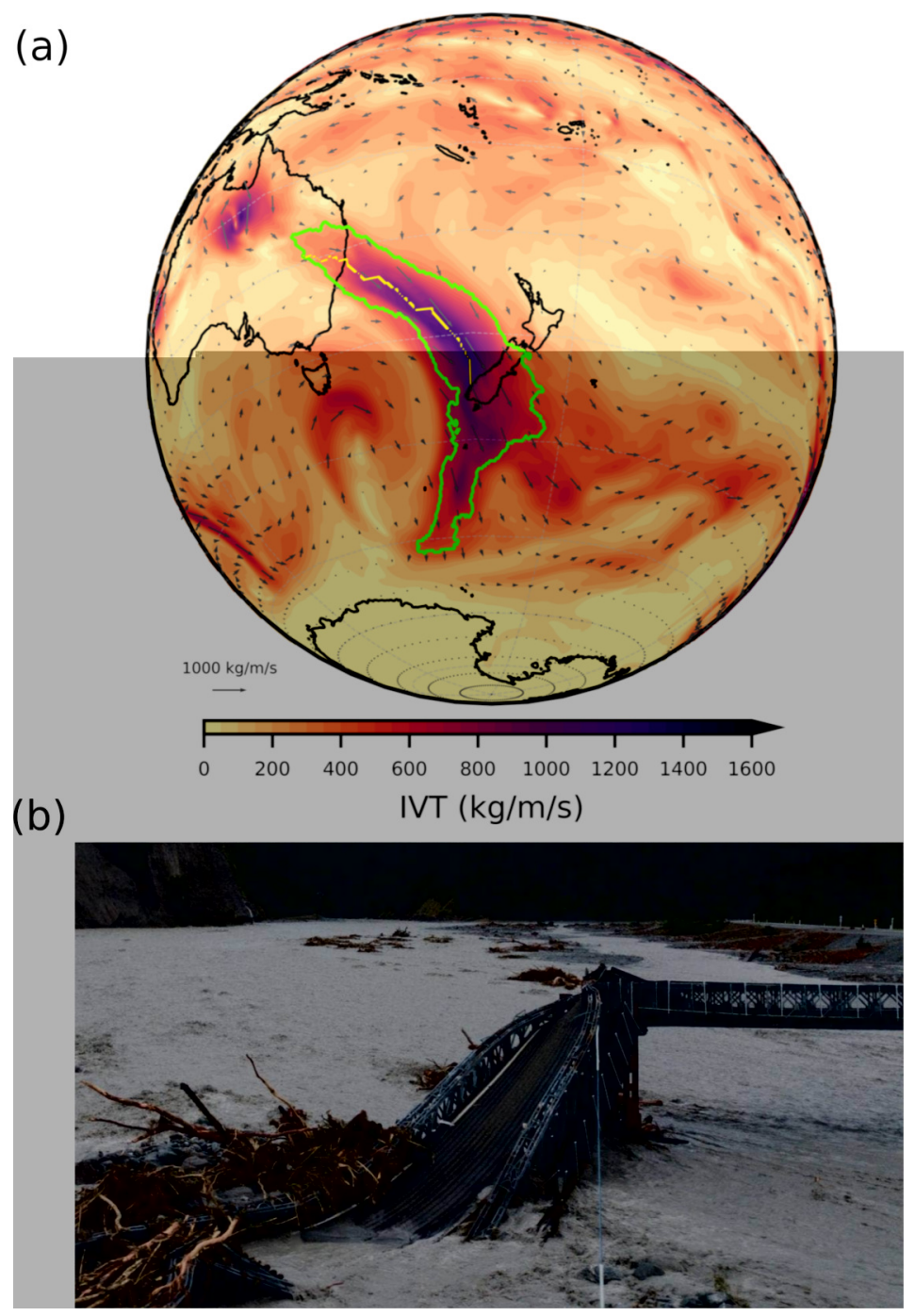

Figure 3.1: (a) Atmospheric river making landfall in New Zealand, 25/03/2019 1800 UTC. Coloured contours and vectors show integrated water vapor transport (IVT) from ERA5. (b) Damage to the Waiho River bridge following the extreme AR event (image by George Heard, 2019). 
longed period of moisture flux and orographic forcing produced event rainfall totals of over a metre for parts of the Southern Alps, with Cropp River recording $1086 \mathrm{~mm}$ of precipitation in 48 hours. Rainfall stations at Haast River, Mueller Hut, Ivory Glacier, Milford Sound, Mt Philistine and Mt Cook all recorded event rainfall totals above $500 \mathrm{~mm}$. Near-record 1-day rainfall totals were also observed at Manapouri, Hokitika, Secretary Island, Greymouth and Arthurs Pass (NIWA Monthly Climate Summary, March 2019). The vertical structure was typical for extreme events in the Southern Alps (Henderson and Thompson, 1999; Cullen et al., 2019), a low-level jet ahead of the cold front, driving a strong inner AR core and strong northwest flow aloft. Over the AR cross section the flow rate of moisture well exceeded the equivalent liquid water flow rate through the mouth of the Amazon and Nile rivers combined (calculated from a WRF simulation of the event, see Appendix). The IVT magnitude and precipitation rates observed during this event are among the most extreme seen in the mid-latitudes (outside of tropical cyclones). 


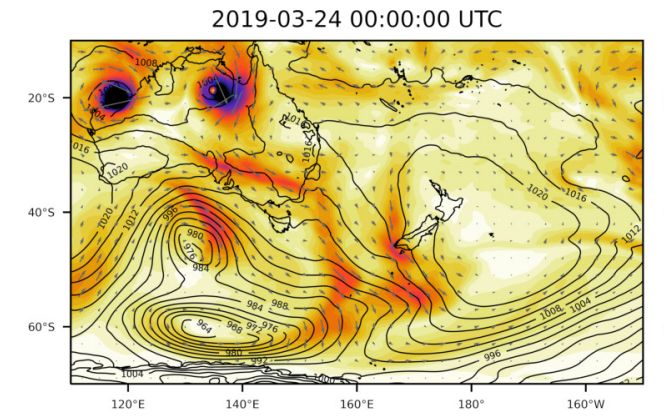

2019-03-25 12:00:00 UTC

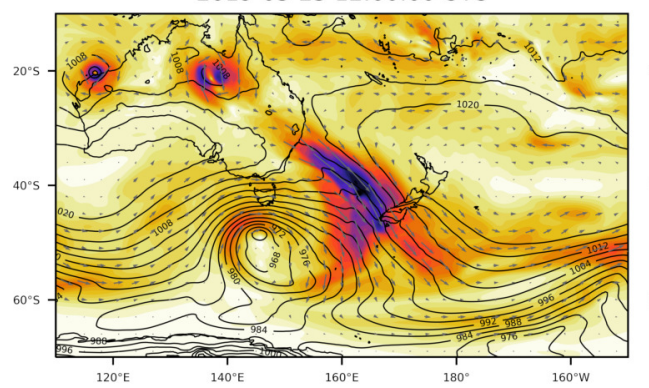

2019-03-27 00:00:00 UTC
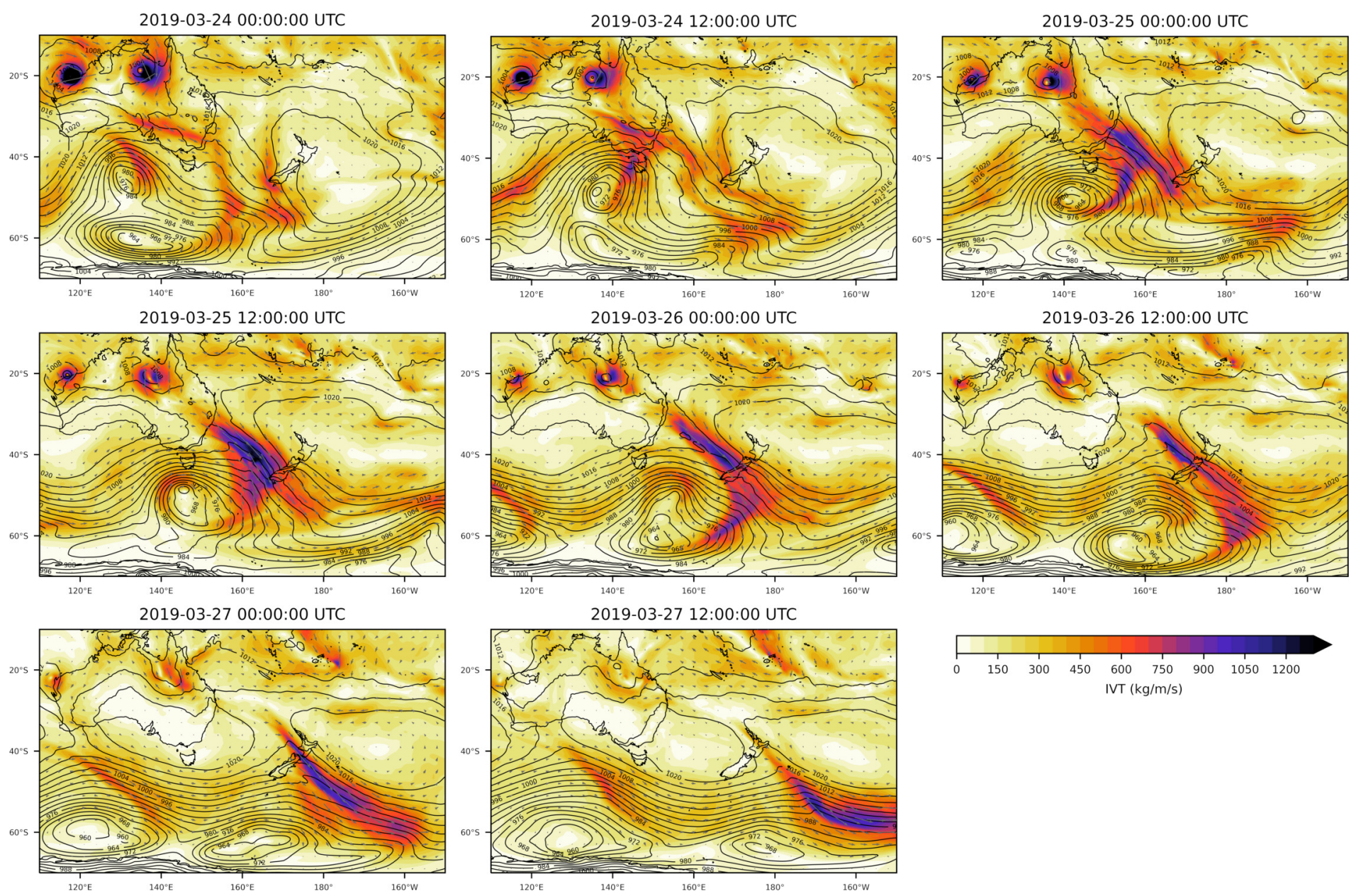

3
0
0
0
0
0
5
0
0
0
0
0
0
0
0
0
0
0
0
0
7
0
0
0
0

Figure 3.2: Synoptic situation for the March 2019 event. Coloured contours and vector arrows depict IVT, black contours show mean sea level pressure.
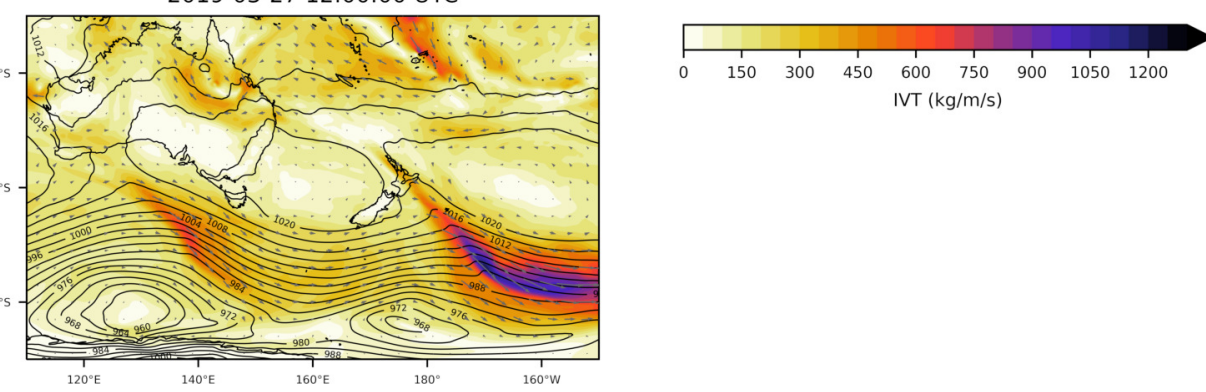
Heavy rainfall in New Zealand is relatively common, and is associated with numerous hazards such as flooding and landslides. New Zealand rainfall extremes are typically associated with low-pressure systems undergoing rapid cyclogenesis ('weather bombs'), as well as tropical cyclones transitioning into ex-tropical cyclones as they move south (approx. one per year (Sinclair, 2002)). For both systems, if sufficiently large and long-lived, the extent of water vapour flux can be sufficient to constitute an atmospheric river. When an AR makes landfall, especially in areas of high topography, it releases much of its water vapour as precipitation through orographic uplift. Further uplift is also forced by cyclonic and frontal lifting (i.e. ascent in the warm conveyor belt), and convective processes (Ralph et al., 2004). These mechanisms can translate the extreme atmospheric moisture flux associated with ARs into damaging hydrological impacts (Neiman et al., 2011; Lavers et al., 2012). Factors such as soil/bedrock type, antecedent soil moisture and land use (including the existence of water storage areas and flood resilient infrastructure) also play a significant role in determining the hydrological impacts of an AR (Albano et al., 2020; Lavers et al., 2020).

In many mid-latitude regions, ARs are found to have significant impacts, contributing to extreme rainfalls and flooding. Summarizing the findings of previous studies: Extreme orographic precipitation associated with ARs has been studied significantly in the Sierra Nevada mountain range in western North America (e.g. Ralph et al., 2006; Dettinger et al., 2011; Rutz et al., 2014). Ralph et al. (2006) performed one of the fist studies linking ARs and extreme flooding events, finding that all seven of the largest flooding events on the Californian Russian River between 1997 and 2006 were associated with ARs. In California, all of the largest storms between 1950 and 2008 (with 3-day precipitation totals exceeding $400 \mathrm{~mm}$ ) were associated with ARs (Dettinger et al., 2011). Across 11 western U.S. states between 1978-2017, a total of US $\$ 42.6$ billion in total damages was attributed to ARs (Corringham et al., 2019). Nayak and Villarini (2017) found that $40 \%$ of the top $1 \%$ daily precipitation totals, and $70 \%$ of annual peak floods, in the central United States were associated with ARs. In Europe, Lavers et al. (2011) found that the 10 largest flood events in the United Kingdom since 1970 were all associated with ARs. Over $90 \%$ of extreme daily precipitation events on the west coast of Norway since 1900 were associated with ARs (Azad and Sorteberg, 2017). In the South American Andes, over half of the top 25\% observed precipitation rates occurred under AR conditions between 2001-2016 (Viale e al., 2018). In South Africa, $70 \%$ of the top 50 daily winter precipitation totals were linked to ARs (Blamey et al., 2018).

The Southern Alps are the most prominent topographic barrier in the Southern Hemisphere south of $40^{\circ}$, experiencing annual precipitation totals of over $10 \mathrm{~m}$ (Kerr et al., 2011). The strongest ARs making landfall on the west coast of the South Island drive intense and prolonged moisture flux toward the Southern Alps, and contribute to 3-day precipitation totals exceeding $1000 \mathrm{~mm}$ (Prince et 
al., 2021). Prince et al. (2021) analyzed data from eight rainfall stations across New Zealand. The strongest AR impacts were observed in the South Island; ARs were found to account for $78 \%$ of total precipitation and $94 \%$ of extreme precipitation on the West Coast (Prince et al., 2021). By investigating observations from eleven New Zealand rainfall stations, Reid et al. (2021) found that between seven and ten of the top ten daily rainfall totals were associated with ARs. Further, nine of the ten most costly floods in New Zealand between 2007 and 2017 were associated with ARs (Reid et al., 2021).

ARs also have significant impacts on New Zealand's cryosphere. Little et al. (2019) found that AR-like structures, with northwesterly circulation and IVT $>1600$ $\mathrm{kgm}^{-1} \mathrm{~s}^{-1}$, were a significant contributor of extreme snowfall and ablation at Brewster Glacier in the Southern Alps. Porhemmat et al. (2021) found that approximately $70 \%$ of large snowfall events (snow depth increase $>90$ th percentile) at selected locations in the Southern Alps coincided with AR landfalls. Glacier mass balance in the Southern Alps is governed by the local atmospheric circulation, namely the airflow direction airflow and air temperature (Fitzharris et al., 1997; Conway and Cullen, 2016; Cullen et al., 2019). Therefore the fate of New Zealand's glaciers, which are sensitive to climate variation and climate change, will be strongly influenced by the frequency and characteristics of future AR landfalls.

The main benefit of the AR framework is its ability to link regions of intense moisture flux with local impacts. Given the large societal impacts of AR events in New Zealand, it is therefore important to quantify the spatial and temporal variability of AR impacts. This chapter investigates the impacts of ARs on New Zealand rainfall. Using a comprehensive AR dataset spanning 40 years (19792019), we assess the impact of ARs on seasonal rainfall totals and extreme events. We extend the analysis of Prince et al. (2021) and Reid et al. (2021) by incorporating 189 stations over the 1979-2019 period in the analysis. To investigate the social and economic costs of AR events, we also investigate the insurance losses associated with extreme AR events since 1980.

\subsection{Data and Methods}

\subsubsection{Daily Rainfall Data}

In order to accurately assess the spatial distribution of AR impacts we use local rain gauge records. Daily rainfall station data is obtained from the New Zealand Climate Database (CLIDB) managed by the National Institute of Water and Atmosphere (NIWA): 
CliFlo: NIWA's National Climate Database on the Web

http://cliflo.niwa.co.nz

Retrieved 24-April-2020

Each station reports 24-hour accumulations of precipitation at 0900 NZST time (2100 UTC Previous Day). The majority of station observations are recorded using automatic tipping bucket rain gauges which measure the time taken to collect every $0.1 \mathrm{~mm}$ of precipitation. Note that precipitation includes snow and hail as rain gauges cannot distinguish between phases. We use daily rainfall observations between 1979 and 2019, to coincide with the AR identification period. Station data was checked for completeness. Stations that were open during the 1979-2019 period, with at least 85\% complete data were retained. This provides a mostly complete dataset over the 1979-2019 period, consisting of 189 stations. Metadata for each station was also retrieved.

A number of standard quality controls were performed on the data. First, the data was checked for any physically impossible values (e.g. negative rainfall totals), no such errors were found. Rainfall accumulations from a period greater than 24 hrs were identified and discarded. Rainfall accumulations greater than $300 \mathrm{~mm}$ were manually checked against weather maps and extreme event records. No unreasonable values were found. No missing data was filled in. It is important to remove non-climate factors from the dataset so that temporal variations are attributed only to climate processes. Non-climate variation may be introduced through relocation of the station, changes to land-use, or changes to the instrument itself. These artificial shifts can significantly bias the analysis of trends, variability and extremes, therefore before calculating precipitation indices, we require homogeneity of the dataset. Homogeneity testing is performed using RHtests_dlyPrcp software (Wang and Feng, 2013). For each station, we test for any significant changepoints in the time series. We test each station for any statistically significant Type- 1 changepoints. Type- 1 changepoints are those identified by applying a maximal F-type test, without including known changepoints from the metadata (Wang, 2008). Artificial shifts in the daily precipitation data series are detected without using a reference series. The confidence level of the test was set to its default value of $95 \%$. If no significant changepoints are identified, the dataset for that station is labeled as homogeneous. Of the 189 stations comprising our daily rainfall dataset, 77 were identified as homogeneous over the 1979-2019 period. We then calculate core indices defined by the Expert Team on Climate Change Detection and Indices (ETCCDI). Due to the complexity involved in adjusting the rainfall data series, precipitation indices were only calculated for stations that were identified as homogeneous. We calculate these indices using the standardised free software, RClimDex (Version 1.9-3), developed by the ETCCDI (Zhang et al., 2018). 


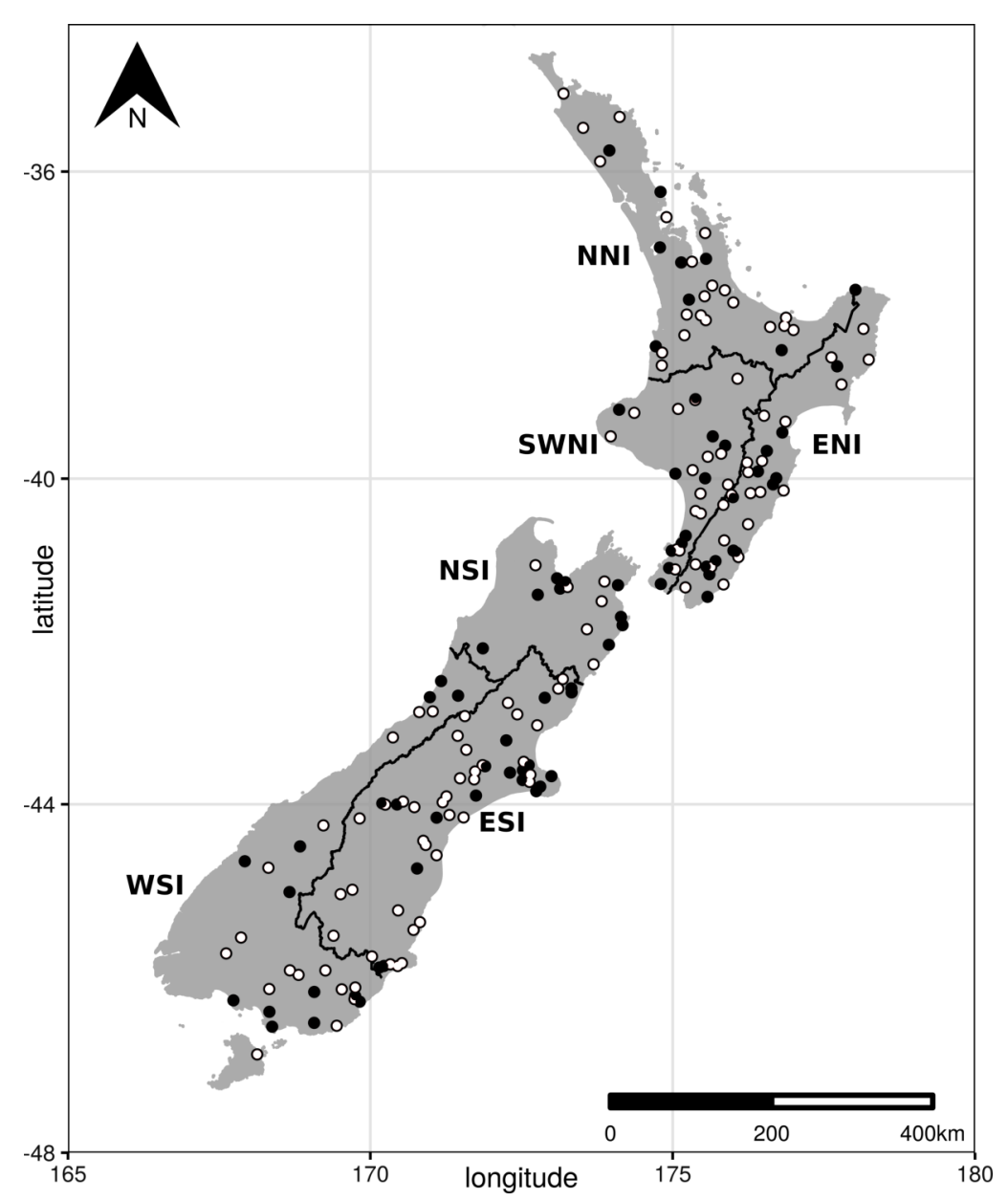

Figure 3.3: The distribution of the 189 stations comprising the daily rainfall dataset, the 74 stations for which we calculate precipitation indices are filled in. The six regional climate zones referred to in this thesis (based on the regions defined by Mullan (1998)) are also shown. 


\subsection{New Zealand Rainfall}

Figure 3.4 shows the mean annual precipitation for 154 stations between 1979 and 2019. The influence of the rain-shadow and orographic enhancement are clearly seen to the east and west of the Southern Alps. The west coast of the South Island is New Zealand's wettest area, while the driest area is directly east of the divide. Annual rainfall totals range from below $500 \mathrm{~mm}$ at Alexandra, to above 3000 $\mathrm{mm}$ for elevated West Coast locations. Mean seasonal rainfall patterns, for 554 stations between 2000-2018, are also shown in Figure 3.5. The North Island and northern South Island receive the highest seasonal rainfall totals during winter, while the eastern South Island, south of Banks Peninsula, receives the highest seasonal rainfall total during summer. The west coast of the South Island receives the highest seasonal rainfall total during spring.

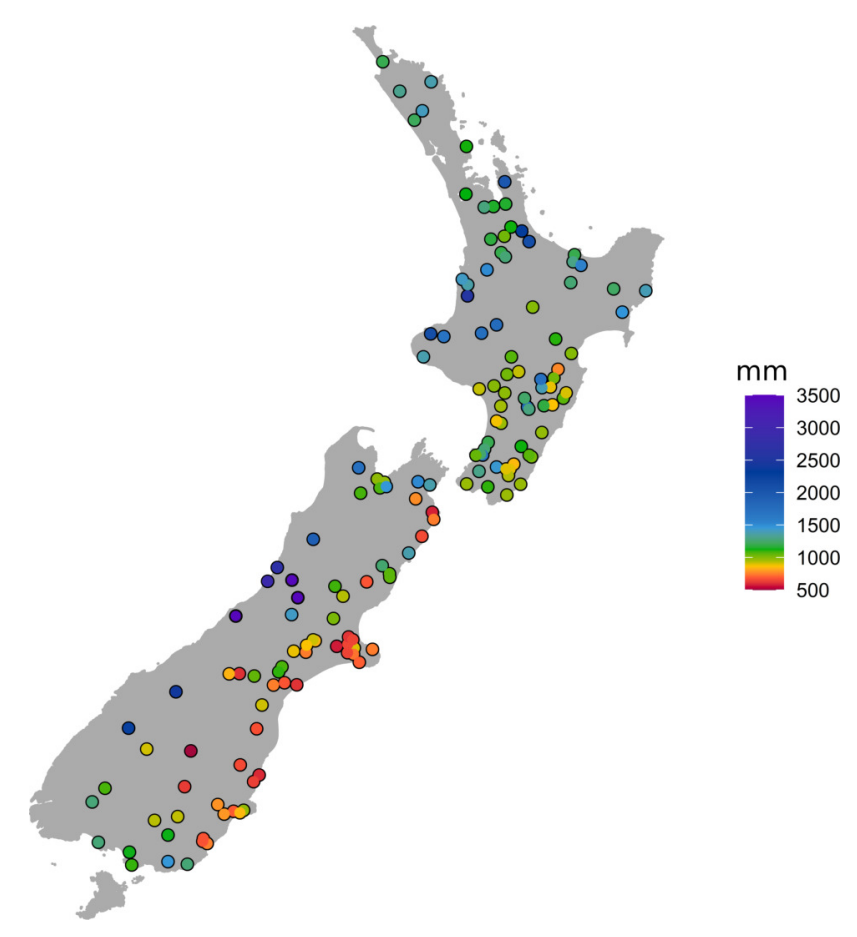

Figure 3.4: Mean annual precipitation for New Zealand stations (19792019). Station data obtained from the New Zealand Climate Database (CLIDB) managed by the National Institute of Water and Atmosphere (NIWA). 

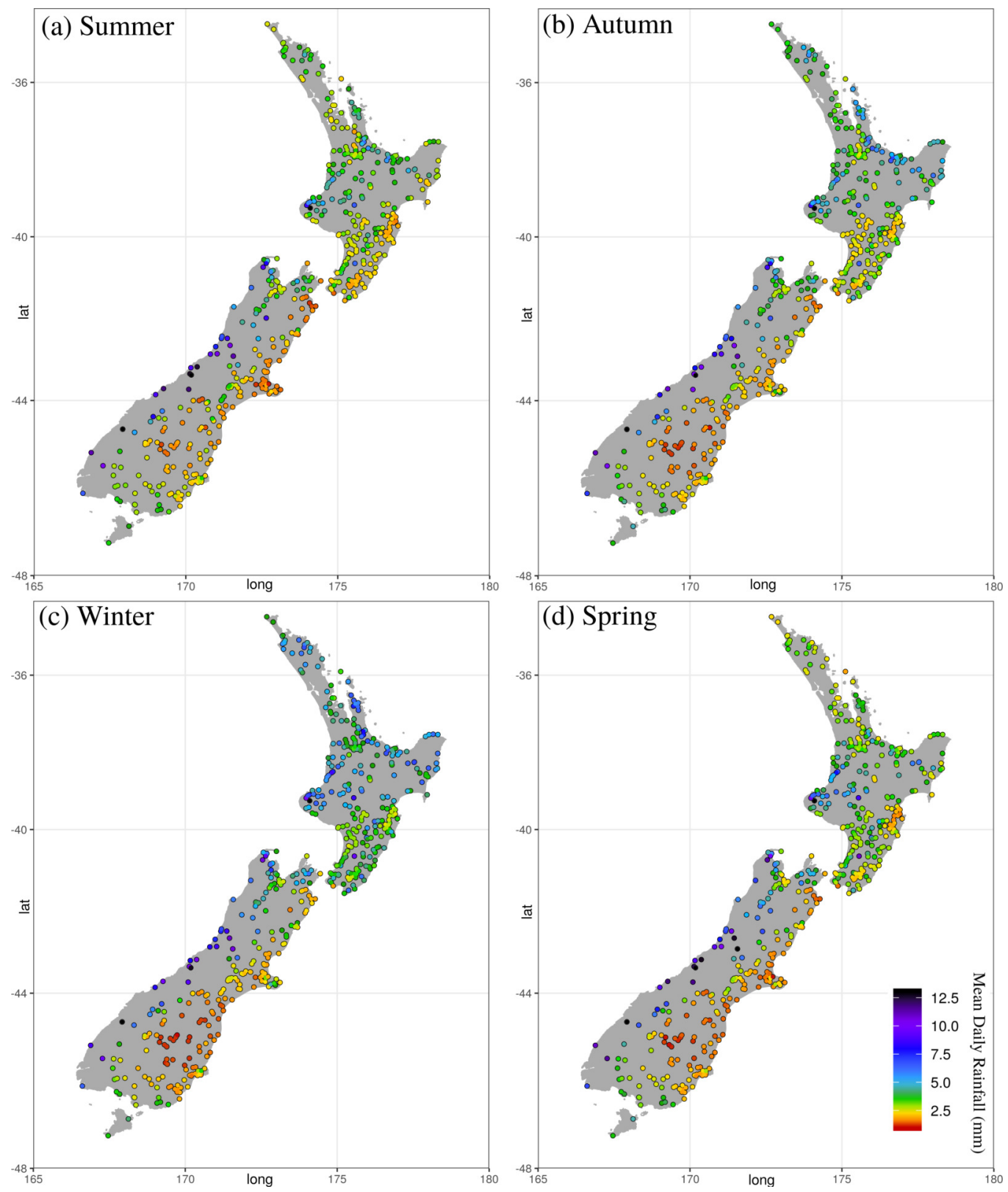

Figure 3.5: Summary of New Zealand seasonal rainfall (2000-2018) based on daily rainfall data from 554 stations. Mean Daily Rainfall for (a) summer (DJF), (b) autumn (MAM), (c) winter (JJA), and (d) spring (SON). 


\subsection{AR Impacts}

At any instant in time, the locations that fall within the AR boundary are defined to be under 'AR conditions'. For each location and each day, if AR conditions exist over that location for at least one time-step (including time-steps 6-hours either side of that day), it is tagged as an 'AR day'. To investigate the impact of ARs on New Zealand rainfall, we calculate the percentage of total annual rainfall falling during AR days. In order to compare directly with other regions of the world, we use the global dataset of Guan \& Waliser (2015). Note that the global algorithm thresholds a comparatively larger AR boundary, thus regions outside the core of the AR are also regarded as under AR conditions. For this reason, Rutz et al. (2019) recommends the use of this type of detection algorithm for attributing hazards such as heavy rainfall, flooding and wind. Figure 3.6 and Figure 3.7 show the annual and seasonal contribution of ARs to total precipitation. In quantifying AR-linked precipitation, the values found here are relatively conservative; AR impacts typically extend outside of the AR boundary and can influence precipitation before it has made landfall, and after the AR has decayed.

There exists a distinct east-west pattern, influenced by the topography, resulting in a rain shadow effect. Due to the prevailing northwesterly moisture flux, the highest contributions are found on the north and west coasts of both islands. Over $45 \%$ of West Coast rainfall fell under AR conditions. Notably, the contributions to northern and western North Island rainfall are on par with values for the west coast of the South Island. This highlights the important role of ARs in replenishing water resources in the north of the country. The smallest contributions (as low as 10\%) were found directly east of the main divide, where orographic rainout reduces links between precipitation and AR landfall. Note that the highest fractions found for New Zealand, on the west coast of the South Island, are on par with values found for western South America (Viale et al., 2018). Using a similar method (though different AR dataset and calculation), Viale et al. (2018) found $40-60 \%$ of annual precipitation in the Chilean Andes was attributed to landfalling ARs, significantly exceeding values found in western North America (Dettinger et al., 2011). Thus, AR contributions to New Zealand rainfall are likely among the highest in the world.

AR-related precipitation fractions did not show significant seasonal variation. The fraction of seasonal AR-related precipitation mostly matched the seasonal frequency of AR occurrence, with the majority of the country experiencing the largest fractions in summer or spring (see Figure 3.9). Note that even in winter, when AR frequency is reduced for the west coast of the South Island, significant AR contributions were observed. This is likely due to the air being closer to saturation, with a weaker winter AR contributing the same rainfall as a strong summer AR. It should be noted that weak summer ARs may fail to fully satu- 


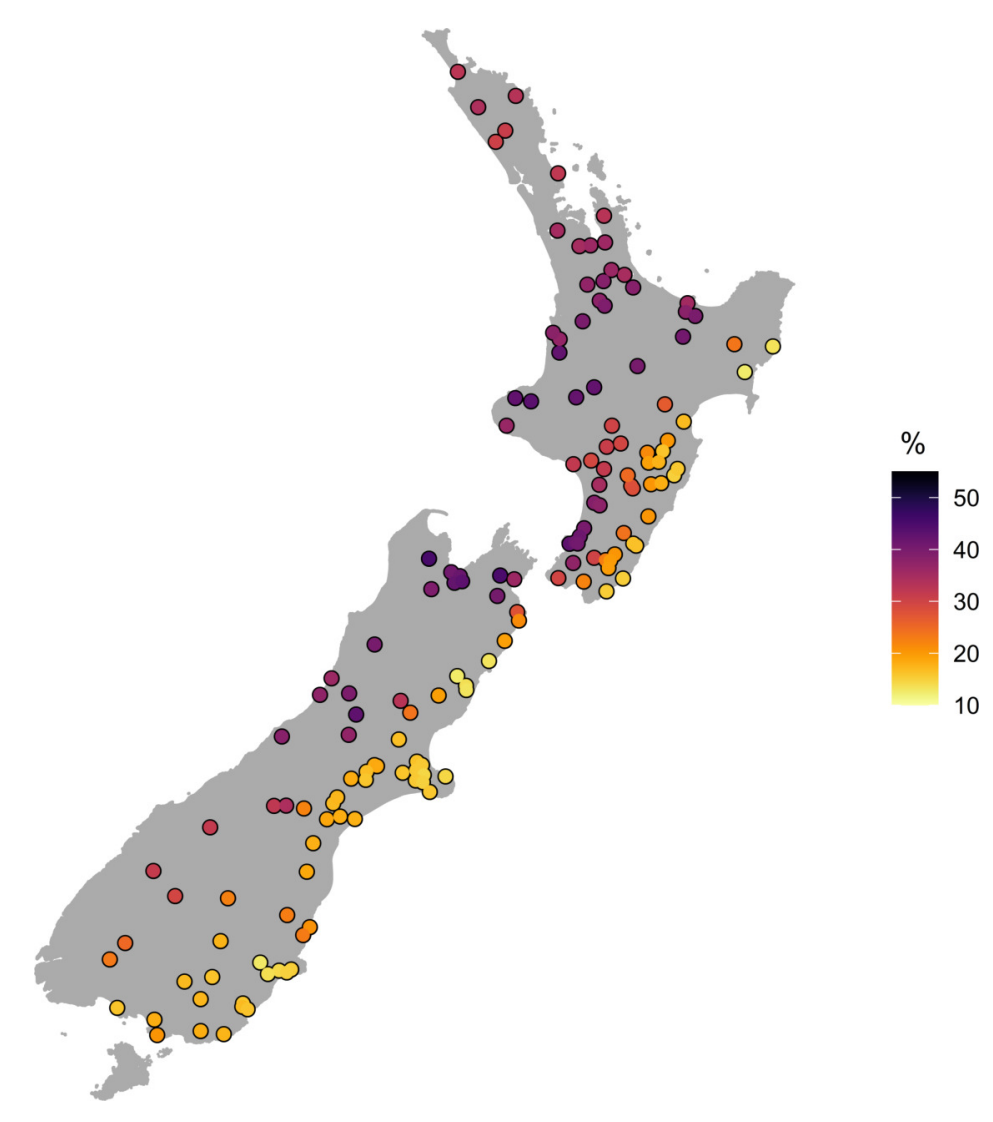

Figure 3.6: Percentage of total annual precipitation associated with AR days for the period 1979-2019. Using Guan \& Waliser (2015) AR dataset, IVT $>85$ th percentile. 
rate the atmospheric column, and thus produce less precipitation, especially at lower elevations (Rutz et al., 2019). Thus, we suspect that the temperature of ARs strongly relates to the observed precipitation accumulations.
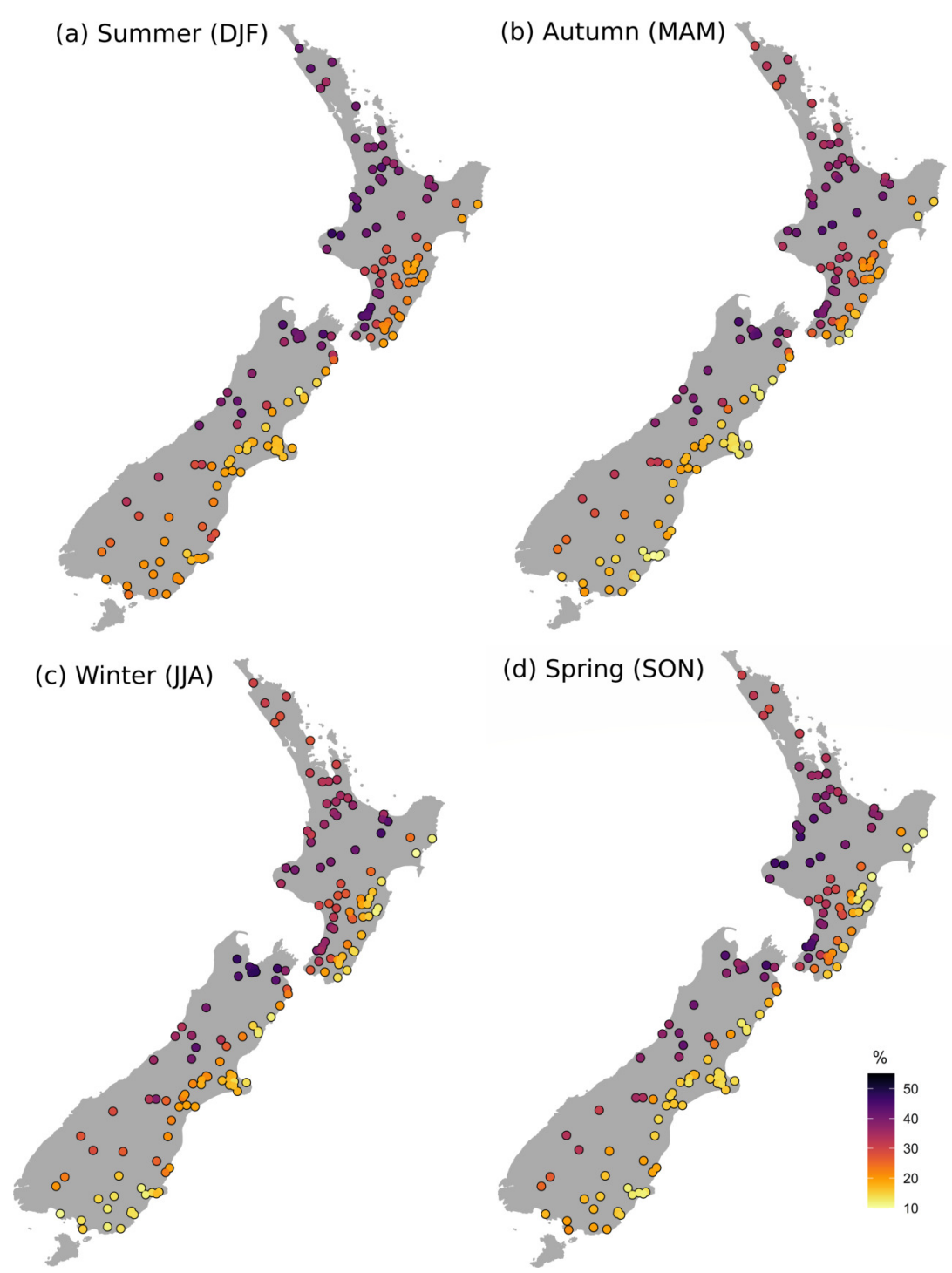

Figure 3.7: Percentage of total season precipitation associated with AR days for the period 1979-2019. Using Guan \& Waliser (2015) AR dataset, IVT $>85$ th percentile.

There exists a strong relationship between instantaneous IVT and precipitation, particularly for elevated locations. Therefore, any time 'AR conditions' occur over 
a location, there is a significant chance of heavy rainfall. In general, IVT values exceeding $750 \mathrm{kgm}^{-1} \mathrm{~s}^{-1}$ are considered notable. Values over $1000 \mathrm{kgm}^{-1} \mathrm{~s}^{-1}$ are considered extreme, and often indicate a significant flooding threat. To further investigate how ARs influence daily rainfall totals, we compare daily rainfall falling under AR versus non-AR conditions. For all wet days ( $>1 \mathrm{~mm}$ rainfall) the median daily precipitation is calculated for AR days and non-AR days. The ratio between these values defines the (AR/non-AR) precipitation fraction. Similar values have been defined in the literature to measure AR precipitation intensity (see for e.g. Neiman et al., 2008; Viale et al., 2018). Aside from the east coast, daily wet-day rainfall totals were at least $50 \%$ higher for AR days. AR/nonAR precipitation fractions were up to 2.5 times higher for West Coast, Taranaki and Waikato locations where orographic forcing can drive appreciable rain-rates. Fractions less than 1 are observed for parts of the South Island east of the main divide, where AR landfalls would typically be associated with reduced moisture due to orographic rain-out (and strong Foehn warming). Looking at the seasonality of the (AR/non-AR) precipitation fractions, a large number of stations were impacted more by winter ARs, mostly stations experiencing higher winter rainfall totals (Figure 3.9). Thus, in general winter ARs tend to be more intense (in terms of total daily precipitation).
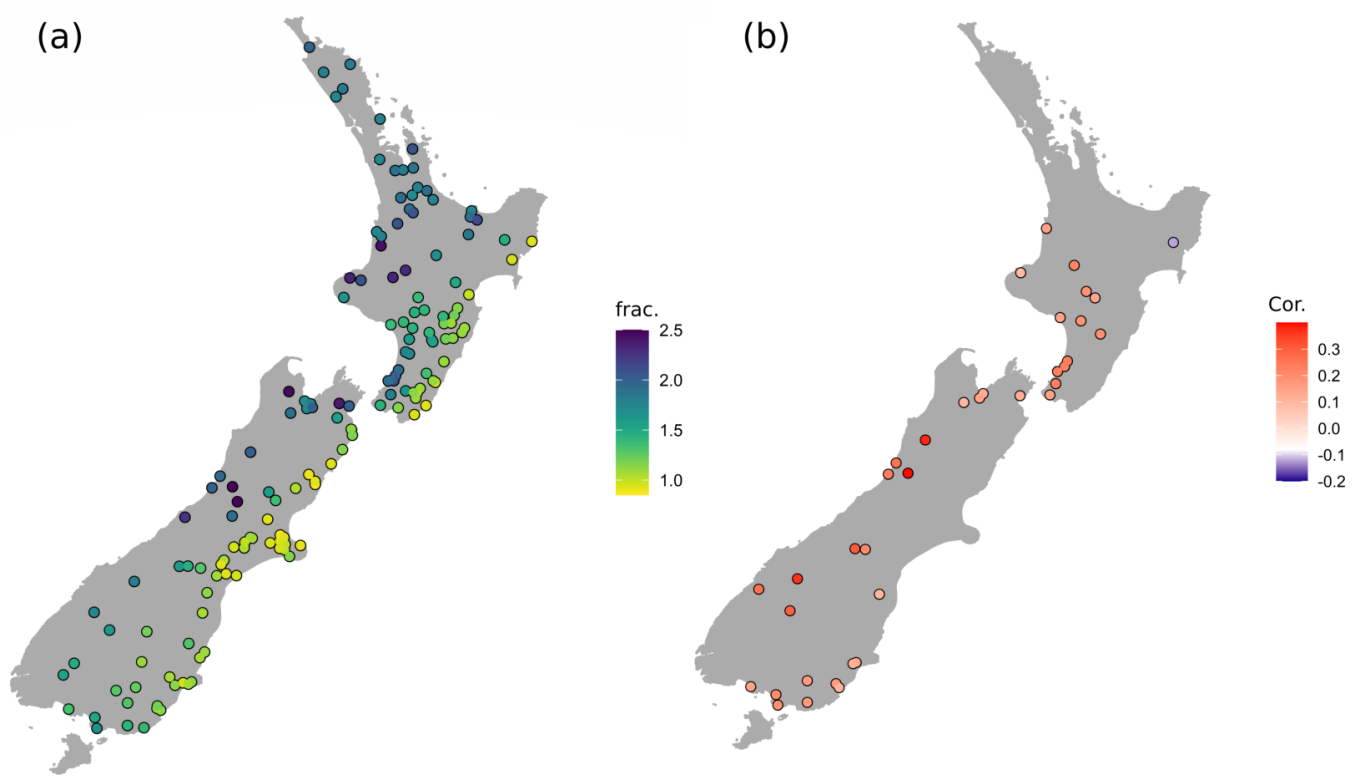

Figure 3.8: (a) Ratio of mean wet-day $(>1 \mathrm{~mm}$ ) precipitation under AR conditions to those under non-AR conditions. (b) Spearman correlation coefficient between monthly AR frequency and maximum 1-day precipitation (RX1DAY), stations significant at $\mathrm{p}<0.05$ shown. 
In the next chapter, we investigate how large-scale climate oscillations influence monthly AR activity in New Zealand. It is implied that an increase in AR frequency corresponds with an increase in heavy precipitation, and an increased likelihood of flooding. While we have demonstrated strong links between AR frequency and accumulated precipitation, it is not clear whether increased AR frequency translates to an increase in the intensity of extreme events (at least on monthly time-scales). To investigate the relationship between AR occurrence and rainfall extremes on monthly time-scales, we compare monthly AR frequency to monthly maximum 1-day precipitation. Figure 3.8(b) shows stations with significant correlations between monthly AR frequency and maximum 1-day precipitation. Only stations in the southern, northern and western South Island and southwestern North Island display significant correlations. This suggests that for the northern North Island and east of both Islands, increases in AR frequency do not necessarily correspond with an increase in the magnitude of extreme rainfall events.

\subsection{Extreme Events}

Flooding events in New Zealand have had major social and economic consequences with losses to property, farmland, livestock, roads and bridges. Flooding is recognized as New Zealand's most frequent and costly natural hazard; insurance industry records show that flooding is the largest source of damage claims from natural disasters (McKerchar and Pearson, 2001). The 12 costliest flooding events in New Zealand between mid-2007 and mid-2017 cost NZ \$472m in insurance damages (Frame et al., 2020). The full economic costs are expected to be higher as these values do not include losses in economic activity in the aftermath of events, or emergency response costs preventing damage to insured property (Frame et al., 2020).

The majority (approx. two-thirds) of New Zealand's population resides in floodprone areas (Rouse et al., 2012), and the number (and value) of insurance payouts associated with flood damage is increasing, with continued development on floodplains (Smart \& McKerchar, 2010). Future flood frequency is recognized as a key risk for New Zealand, though there is some uncertainty in projected changes to extreme rainfall (Reisinger et al., 2014). Current extreme rainfall projections in New Zealand under a local $2^{\circ} \mathrm{C}$ warming suggests that 100 -year extreme events (i.e. events with a 1 in 100 chance of being equaled or exceeded in any given year) could occur approximately twice as often (Bell et al., 2017). Drought frequency in parts of New Zealand is also expected to increase; "time spent in drought in eastern and northern New Zealand is projected to double or triple by 2040" (Reisinger et al., 2014).

Figures 3.10 and 3.11 show a number of recent AR events that were associated 

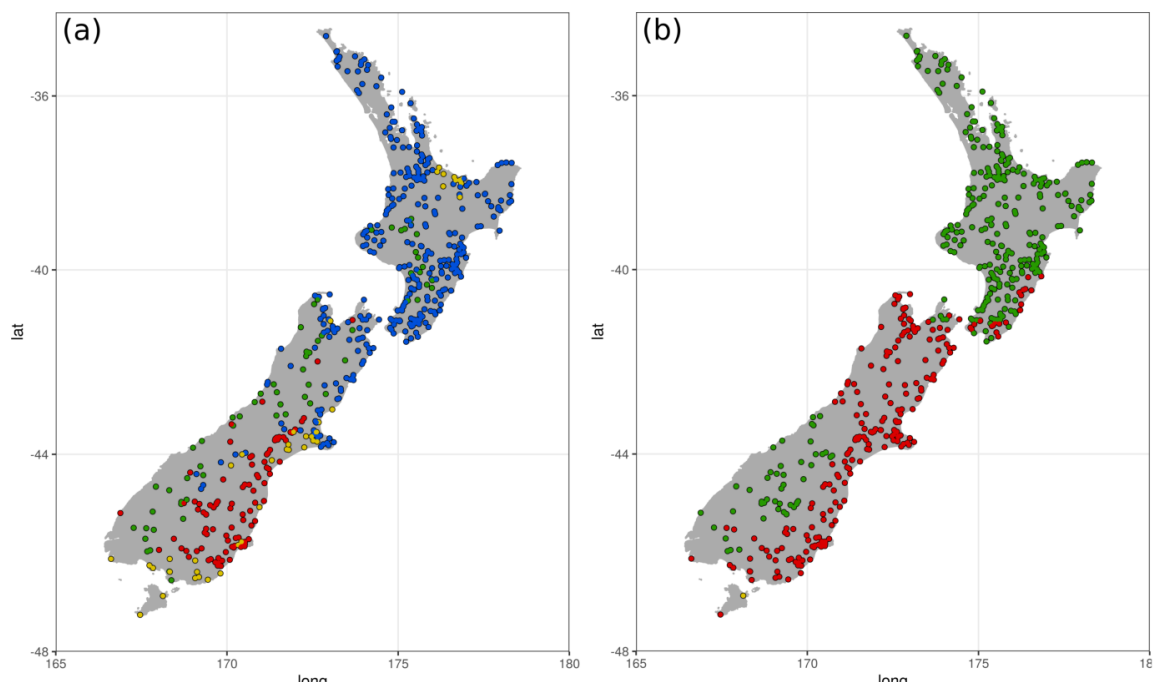

Season

-Summer

- Autumn

-Winter
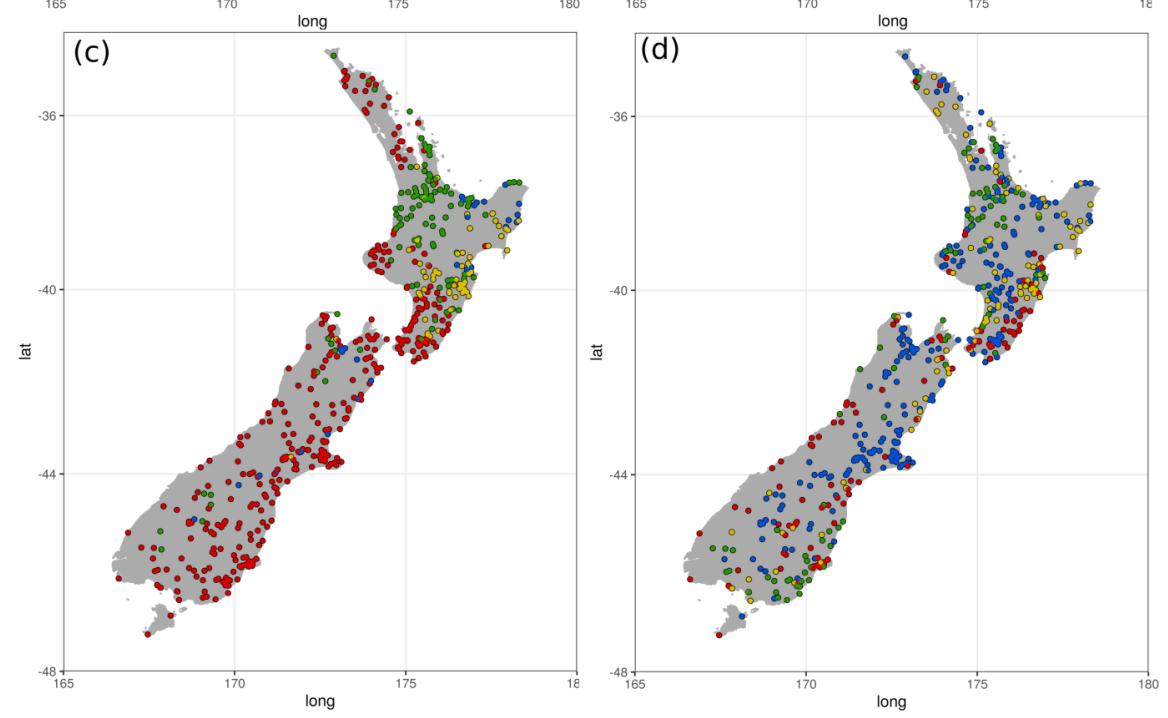

- Spring

Figure 3.9: Season of maximum (a) accumulated precipitation, (b) AR frequency, (c) fraction of precipitation from ARs, (d) AR/non-AR wet-day precipitation fraction. Summer (red), Autumn (orange), Winter (blue), Spring (green). Derived for 554 stations (2000-2018) using the NZ AR detection algorithm 
with significant damages. The events were associated with major damages to infrastructure, road closures and a number of record rainfall totals. Information on impacts was sourced from NIWA Monthly Climate Summaries, niwa.co.nz/ climate/summaries.

To investigate AR contributions to extreme precipitation at each station, we rank the top daily rainfall totals defined by percentile thresholds. We use the 95th and 99th percentiles, consistent with IPCC extreme precipitation indices and a number of previous studies in New Zealand (Griffiths, 2007; Dravitzki and McGregor, 2011). Figure 3.12 shows AR contributions to heavy and extreme precipitation. We find that AR days are associated with up to $70 \%$ of daily rainfall totals above the 99th percentile.

Metservice, New Zealand's authorised provider of severe weather alerts, will issue a severe weather warning whenever there is an expectation of widespread (over an area of 1000 square kilometres or more) rainfall greater than $100 \mathrm{~mm}$ within 24 hours. For each station, we investigate the fraction of daily rainfall observations above $100 \mathrm{~mm}$ that were associated with ARs. If an AR was detected on the day before, during or after the rainfall record (either by the global detection algorithm or NZ detection algorithm) we consider that rainfall total to be associated with an AR. Overall, 79\% of all daily rainfall totals exceeding $100 \mathrm{~mm}$ were associated with an AR. In particular, we find that all daily rainfall totals above $100 \mathrm{~mm}$ for the majority of western and southern South Island stations occurred within one day of an AR (Figure 3.13). Interestingly, a number of stations in the eastern North Island and near Christchurch did not show any association between ARs and daily rainfall totals above $100 \mathrm{~mm}$.

Previous investigations of New Zealand ARs have found that the seasonality of extreme AR events tends to match the general seasonality of ARs. In the South Island, Prince et al. (2021) found that category 4 and 5 AR events were most frequent between November and April, while there is limited seasonality in the number of category 4 and 5 AR events at North Island locations (Prince et al., 2021). By categorizing extreme ARs in terms of observed precipitation (as opposed to IVT intensity and duration), we find that winter ARs are associated with a comparable number of extreme daily rainfalls as summer ARs. Of the daily rainfall totals exceeding $100 \mathrm{~mm}$ that were identified, 633 occurred in summer, 551 events in autumn, 447 events in winter, and 394 in spring. Of these, ARs were associated with $80 \%$ of summer, $72 \%$ of autumn, $84 \%$ of winter, and $84 \%$ of spring events. Thus, while cool-season ARs are less frequent in New Zealand overall, the fraction of extreme events associated with ARs is mostly the same for all seasons. 


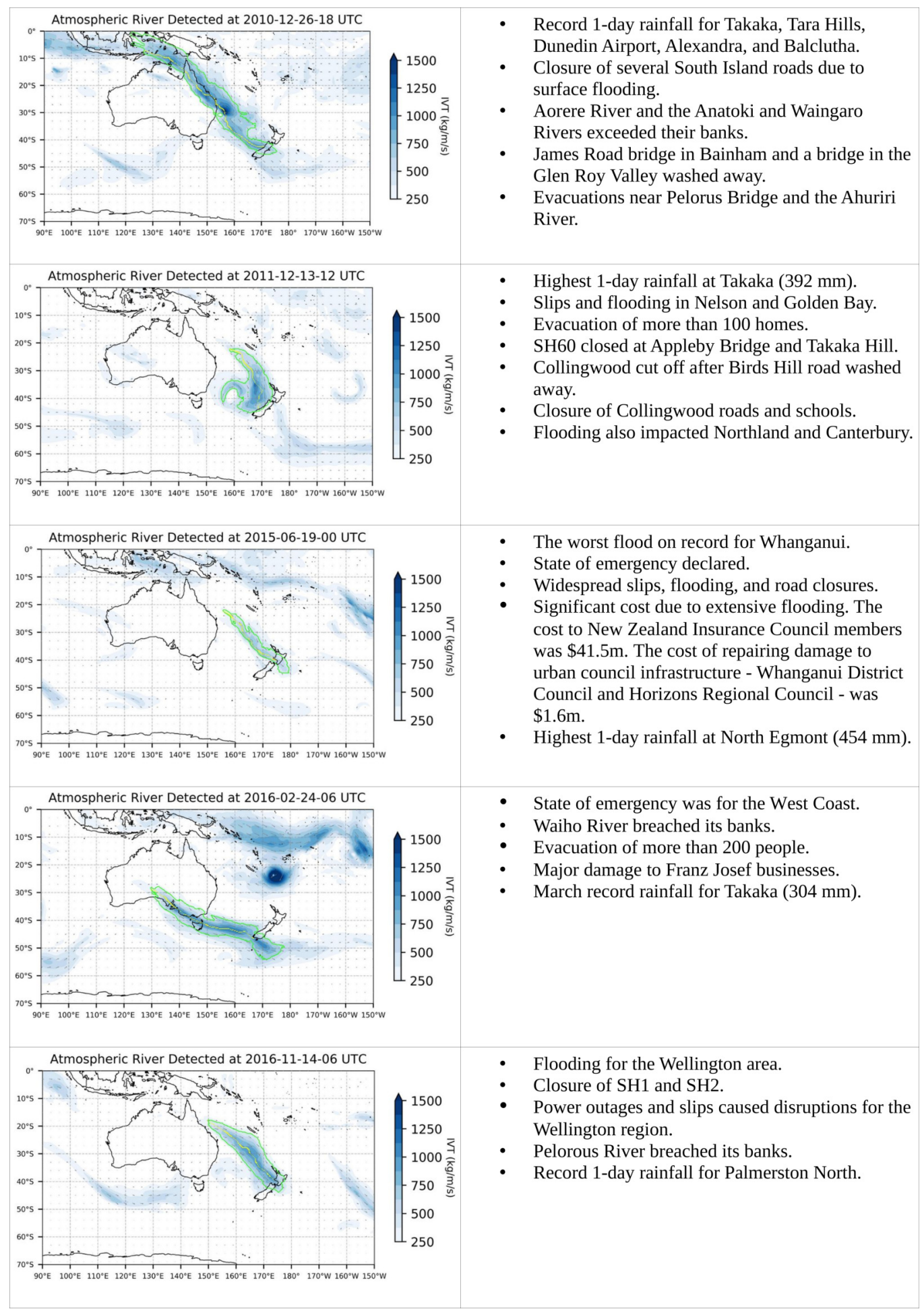

Figure 3.10: A selection of recent extreme AR events associated with notable societal/economic impacts. ARs identified by NZ detection algorithm. Information sourced from NIWA Monthly Climate Summaries. 


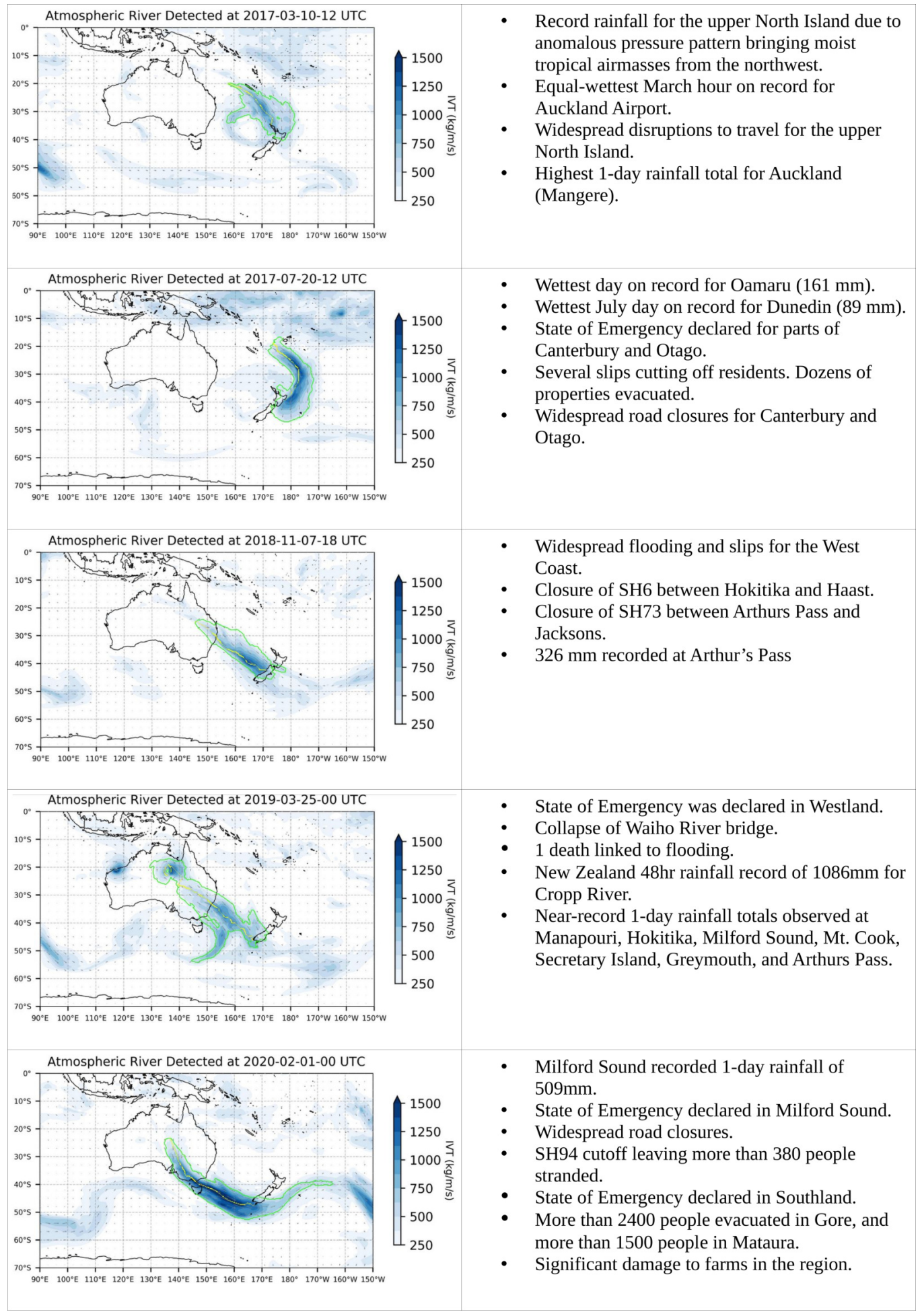

Figure 3.11: A selection of recent extreme AR events associated with notable societal/economic impacts. ARs identified by NZ detection algorithm. Information sourced from NIWA Monthly Climate Summaries. 

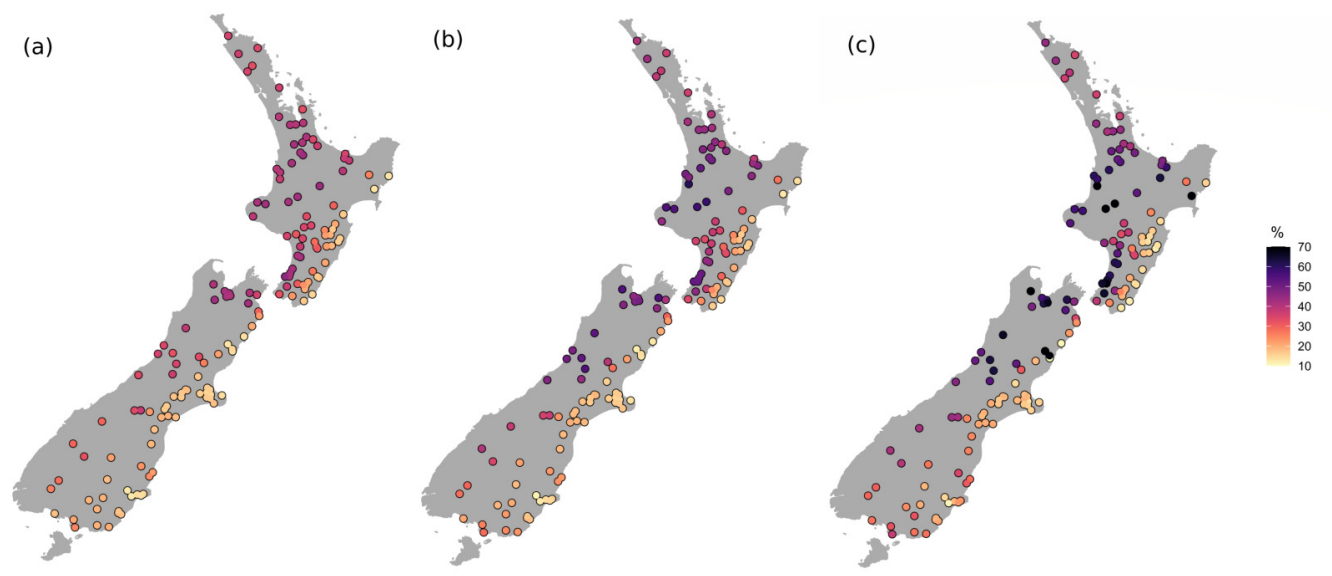

Figure 3.12: Fraction of (a) $>10 \mathrm{~mm}$, (b) $>95$ th percentile and (c) $>99$ th daily rainfall totals under AR conditions.

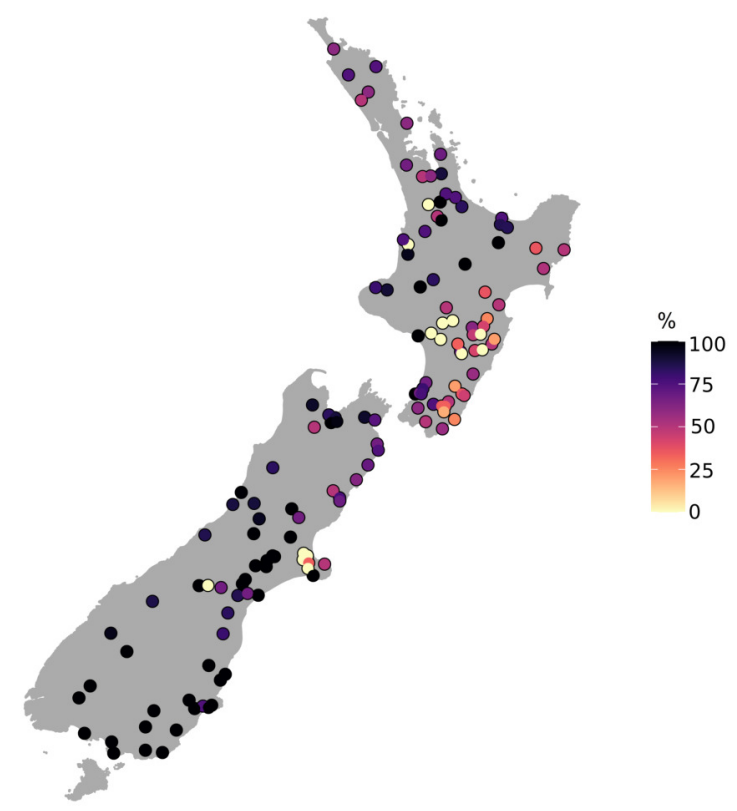

Figure 3.13: Percentage of daily rainfall totals exceeding $100 \mathrm{~mm}$ that are associated with an AR.

While significant AR-related rainfall totals are observed throughout New Zealand, the synoptic patterns that drive these ARs are different for each region. Figure 3.14 depicts the events associated with the five highest daily rainfall totals falling 
under AR conditions recorded in each region. We find that ARs of tropical origin contribute to a large fraction of the highest daily rainfall totals, except for the western South Island where strong and continuous water vapour flux associated with northwesterly flow leads to the highest totals. For the North Island and northern and eastern South Island, extremes tend to be associated with ARs with a more meridional component. Although ARs are typically weakened over east coast stations due to the rain shadow effect, ARs making landfall directly on the east coast can lead to significant rainfall totals. Prince et al. (2021) found that extreme AR events (category 4 and 5 ARs) are most frequent off the coast of New Zealand, to the northeast and south of the country. Indeed, we find that extreme AR events are typically associated with elevated IVT to the northeast of the country (for northern and eastern regions), as well as northwest-oriented ARs extending over the Tasman Sea forced by strong northwesterly flow (for western regions). Over the New Zealand mainland, we find that the frequency of 'extreme' AR conditions peaks in the western South Island, near Hokitika (with >11 'extreme' ARs per year). 


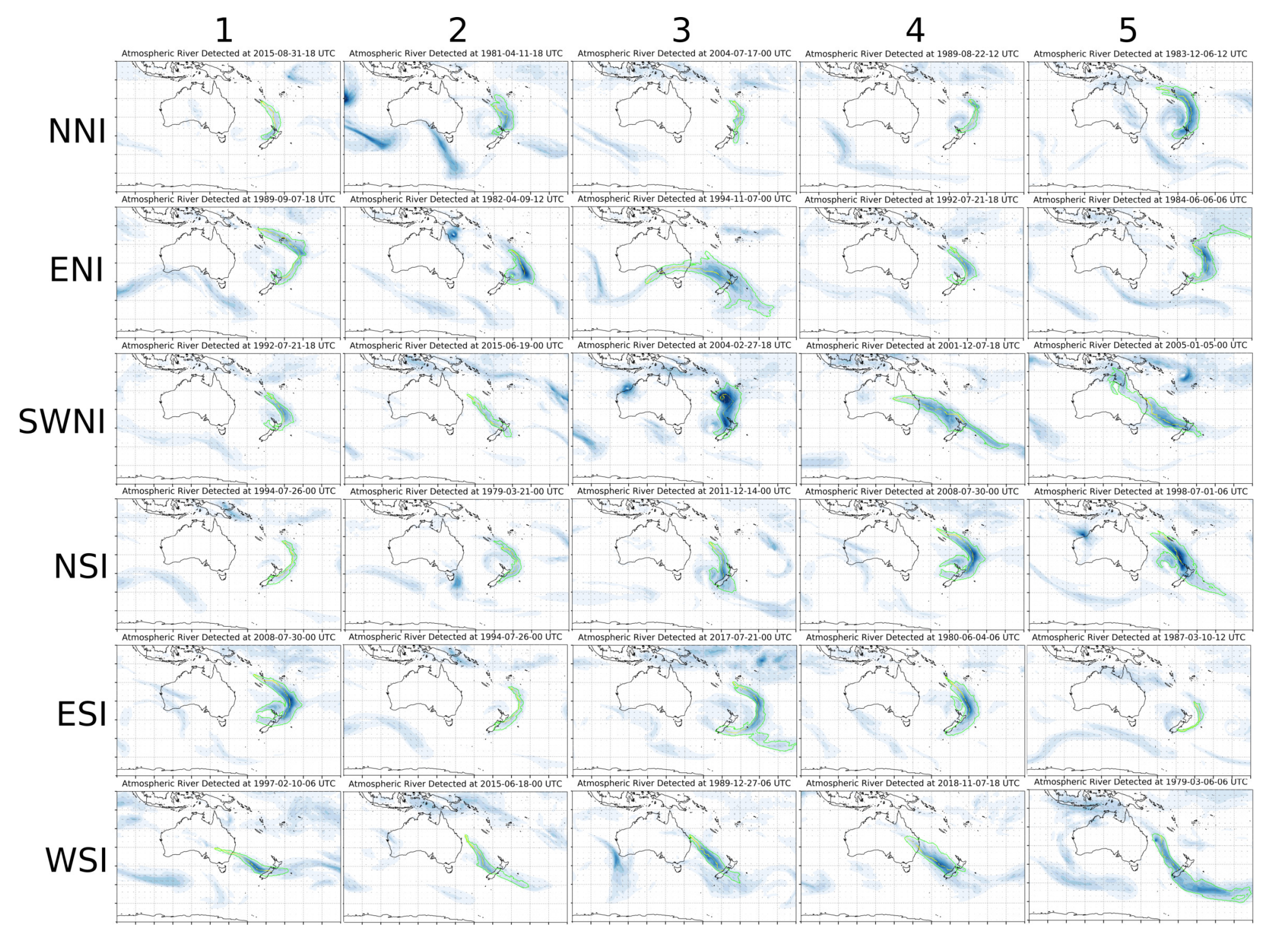

Figure 3.14: ARs contributing to the five highest AR-related daily precipitation totals in each region. 
Between 1979 and 2019, New Zealand experienced more than 140 severe weather events costing millions in insurance claims. We investigate the number of costly severe weather events associated with ARs since 1980 using cost estimates from the Insurance Council of New Zealand (ICNZ), which began keeping records in 1968. Data was obtained from https: / www.icnz.org.nz/natural-disasters/ cost-of-natural-disasters/. Costs were adjusted for inflation, with the consumers price index (CPI) calculated as at 30 June 2017. All events mentioning storms, flooding and severe weather were considered. We consider an event to be associated with an AR if a landfalling AR was detected on the day before, during or after the event date (either by the global detection algorithm or NZ detection algorithm). Figure 3.1 outlines the 25 costliest severe weather events in New Zealand since 1980 . Overall, approximately $63 \%$ of the recognized severe weather events were associated with an AR. Since 1980, the total cost of AR-related damages exceeds NZ $\$ 1.4$ billion. Of the severe weather events identified, 40 occurred in summer, 40 events in winter, 36 events in autumn, and 24 in spring. Of these, ARs were associated with $67.5 \%$ of summer, $50 \%$ of autumn, $72.5 \%$ of winter, and $58.3 \%$ of spring events. Thus ARs may be the dominant driver of extreme winter rainfall totals.

Table 3.1: 25 costliest flooding events in New Zealand since 1980 (costs based on best estimates from the Insurance Council of New Zealand). We show if an AR was detected by the global or NZ detection algorithm over the duration of each event.

\begin{tabular}{|c|c|c|c|c|c|c|}
\hline Start Date & End Date & Event & Inflation Adjusted Cost (Sm) & AR (Global) & AR (NZ) & AR \\
\hline $2004-02-15$ & $2004-02-16$ & Storm Damage - Lower Nth Island & 148.3 & NO & NO & NO \\
\hline 1984-01-01 & 1984-01-01 & Invercargill / Southland Floods & 147.7 & NO & NO & NO \\
\hline $2017-04-03$ & \begin{tabular}{|l|}
$2017-04-07$ \\
\end{tabular} & Cyclone Debbie remnants & 91.46 & YES & NO & YES \\
\hline $2013-09-11$ & \begin{tabular}{|l|}
$2013-09-12$ \\
\end{tabular} & Nationwide storm & 77.1 & YES & YES & YES \\
\hline $2018-04-10$ & 2018-04-11 & Severe weather including tornadoes & 74.4 & YES & NO & YES \\
\hline $2007-07-10$ & $2007-07-12$ & Storm event - Far North / Auckland / Coromandel & 72.7 & NO & YES & YES \\
\hline $1988-03-08$ & $1988-03-08$ & Cyclone Bola & 72.1 & NO & NO & NO \\
\hline 1999-12-01 & $1999-12-01$ & Queenstown Lakes District Floods & 67.6 & YES & YES & YES \\
\hline $2017-03-07$ & $2017-03-12$ & Upper North Island flooding & 61.7 & NO & NO & NO \\
\hline $2014-04-17$ & \begin{tabular}{|l|}
$2014-04-17$ \\
\end{tabular} & Easter Weekend Storm and Floods & 56.9 & YES & YES & YES \\
\hline $2010-11-28$ & $2010-11-28$ & Southland Storm & 54.5 & NO & NO & NO \\
\hline $2008-07-30$ & $2008-08-01$ & Nationwide Storms & 53.2 & YES & YES & YES \\
\hline $2006-06-12$ & $2006-06-12$ & Storms North \& South Islands & 52.3 & YES & YES & YES \\
\hline 2013-04-19 & 2013-04-22 & Nelson/Bay of Plenty storm and floods & 48.2 & NO & NO & NO \\
\hline $1986-03-13$ & \begin{tabular}{|l|}
$1986-03-13$ \\
\end{tabular} & North Otago / South Canterbury Floods & 46.5 & NO & $\mathrm{NO}$ & NO \\
\hline 2018-02-01 & \begin{tabular}{|l|}
$2018-02-01$ \\
\end{tabular} & Cyclone Fehi & 45.9 & YES & NO & YES \\
\hline 2015-06-18 & $2015-06-21$ & Flooding and Storm Lower North Island including Whanganui & 42.4 & YES & YES & YES \\
\hline $2013-06-20$ & \begin{tabular}{|l|l|}
$2013-06-22$ \\
\end{tabular} & Nationwide storms & 41 & NO & NO & NO \\
\hline $1980-06-05$ & $1980-06-05$ & Taieri / Otago / New Plymouth Floods & 38.7 & YES & YES & YES \\
\hline 2014-06-09 & 2014-06-11 & Severe Weather North and South Islands & 38.6 & YES & NO & YES \\
\hline $2018-02-20$ & \begin{tabular}{|l|}
$2018-02-20$ \\
\end{tabular} & Ex-Tropical Cyclone Gita & 35.6 & NO & YES & YES \\
\hline 2018-01-04 & \begin{tabular}{|l|}
$2018-01-07$ \\
\end{tabular} & Nationwide severe weather & 34.2 & YES & YES & YES \\
\hline $2017-07-20$ & \begin{tabular}{|l|}
$2017-07-22$ \\
\end{tabular} & South Island flooding & 31.2 & YES & YES & YES \\
\hline $2016-03-23$ & \begin{tabular}{|l|}
$2016-03-24$ \\
\end{tabular} & Flooding and Wind - North and South Islands & 30.9 & YES & NO & YES \\
\hline $2008-07-26$ & $2008-07-27$ & North Island Storms & 30.7 & NO & YES & YES \\
\hline
\end{tabular}

Although a significant number of extreme rainfall events in New Zealand are associated with ARs, not all ARs contribute to severe weather impacts. Atmo- 
spheric Rivers also provide beneficial precipitation for New Zealand by replenishing water resources. In New Zealand, the regions highly impacted by ARs are also important sources of New Zealand's hydroelectricity: the Upper Waitaki River Basin accounts for 30\% of New Zealand's hydroelectricity (Caruso et al., 2013), and the Waikato region accounts for $13 \%$ of New Zealand's total electricity (Dravitzki and McGregor, 2011). Therefore, the AR framework may be particularly useful in providing long-range forecasts for reservoir management and hydroelectricity production. Indeed, in the western United States, AR research was primarily driven to serve the reservoir management and hydroelectric power generation sectors (Ralph et al., 2017).

Figure 3.15 shows the year-to-year variability in precipitation for New Zealand stations. Regions with higher variability in rainfall, fewer wet days, and more frequent dry periods are most prone to droughts. In particular, the Canterbury Plains and Northland have been identified as regions most vulnerable to future drought frequency increase (Clark et al., 2011). The large variability in precipitation in these regions is due to the small number of storms that contribute the majority of annual precipitation. At a number of east coast stations, fewer than 15 days account for more than half of the total annual precipitation. Therefore a year with slightly fewer beneficial storms can have significant impacts, reducing annual precipitation significantly and contributing to drought events. Thus, much of the annual variability in precipitation can be attributed to the number of ARs making landfall each year, and strong correlations exist between a lack of landfalling ARs and regional drought events. In a global study, Paltan et al. (2017) noted that the absence of ARs can increase the occurrence of hydrological droughts by up to $90 \%$. For New Zealand, it was noted that ARs may contribute up to $80 \%$ to low and high river flows (Paltan et al., 2017). A number of factors can contribute to droughts in New Zealand, including increased frequency of anticyclones and blocking highs over the country, a lack of moist northerly air flows (associated with tropical variability e.g. ENSO and MJO), and a persistent positive Southern Annular Mode (Salinger and Porteous, 2014). For the north of the country in particular, the difference between continued drought and beneficial rain is likely determined by the landfall location of ARs.

Although not expanded upon in this thesis, we find that AR frequency is strongly negatively correlated with standard drought indices such as the Standardised Precipitation Index (SPI) and consecutive dry days (CDDs), particularly for northern New Zealand stations. Drought events (identified using the SPI index) at North Island stations were associated with a continuous band of reduced AR frequency extending from the Java Sea to the Chatham Islands (with significantly fewer ARs over northern Australia). Drought events at eastern South Island stations were associated with decreased AR frequency to the east of the North Island, and increased AR frequency south of New Zealand. Drought events in the west- 

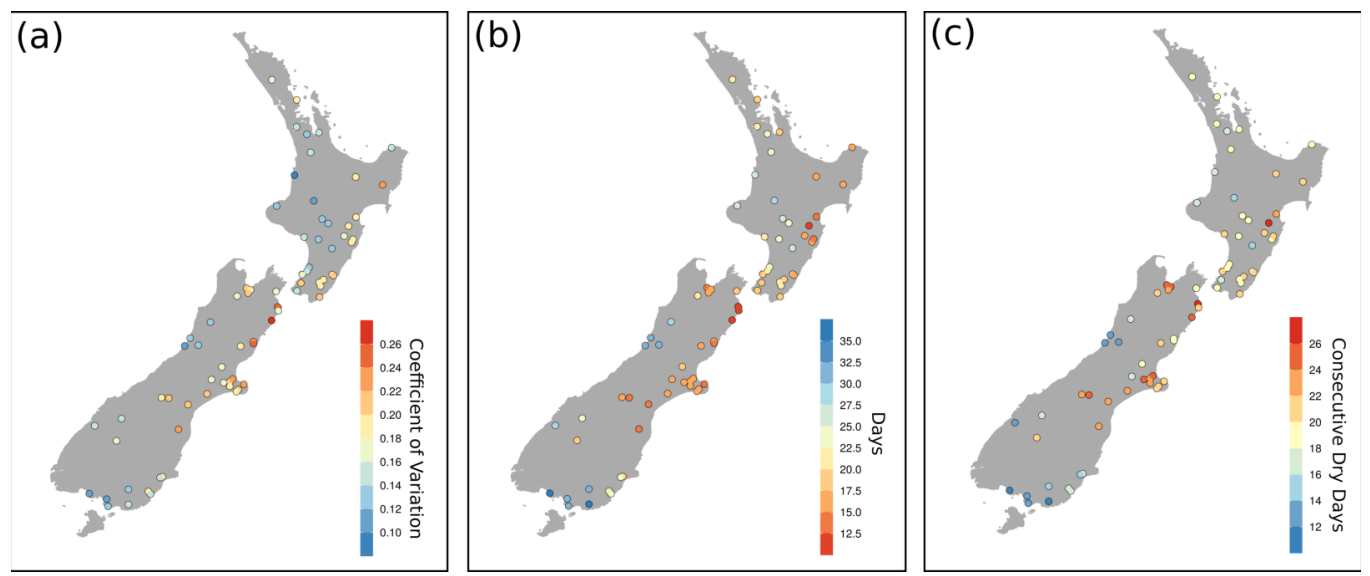

Figure 3.15: Precipitation variability in New Zealand. (a) Coefficient of variation of total annual wet-day precipitation. (b) Minimum number of wet days per year providing half of the total annual precipitation. (c) Mean number of maximum consecutive dry days (PRCP $<1 \mathrm{~mm}$ ) per year.

ern South Island showed a similar AR frequency pattern to the positive phase of the Southern Annular Mode. Drought frequency and intensity is expected to increase due to climate change, with the northern and eastern North Island and the eastern South Island at particular risk (Reisinger et al., 2014). A reduction in mean rainfall, particularly in spring and summer, and increased average temperatures is expected to contribute to severe drought impacts in these regions (Salinger and Porteous, 2014; Reisinger et al., 2014). Thus, the relationship between drought and AR frequency warrants further investigation. In particular, further research on how various climate oscillations influence current and future New Zealand drought may benefit from analysis of ARs. We will investigate how large-scale climate drivers influence AR frequency in New Zealand in the next chapter.

\subsection{Conclusions}

Given the large societal impacts of AR events in New Zealand, it is important that we understand the spatial and temporal distribution of AR-related precipitation. The aim of this chapter was to quantify the importance of ARs to New Zealand rainfall, particularly their association with New Zealand flooding events (compared to other flood producing mechanisms) and identify the regions most susceptible to AR impacts. Due to the complexity of New Zealand's orography, a distinct east-west pattern is observed, with ARs contributing significantly to annual rainfall totals in the northern and western regions of both islands. In 
these regions, over $45 \%$ of rainfall fell directly under AR conditions, contributing to daily rainfall totals 2.5 times higher on average compared to non-AR days. Further, we found that AR days were associated with up to $70 \%$ of daily rainfall totals above the 99th percentile. The results are comparable to those found in North America, South America and Europe, which have found strong connections between AR events and extreme rainfall. In the Southern Alps, these extreme AR events likely contribute to the highest AR-related rainfall totals observed in the world. Interestingly, we find that AR contributions to northern and western North Island rainfall are on par with values for the west coast of the South Island. Thus, although ARs are less frequent overall, they play an important role in replenishing water resources in the north of the country. These results suggest that previous investigations of New Zealand ARs may have underestimated the number of AR landfalls (and impacts) in the North Island (particularly the Far North). As this is likely due to the geometry constraints, we suggest use of AR detection algorithms employing both higher spatial resolution and higher IVT threshold for future New Zealand studies.

We find significant damages (exceeding NZ \$1.4 billion since 1980) associated with extreme AR events, with past events having destroyed key infrastructure and property, and even resulted in the loss of life. Thus ARs have sizable implications for the planning of infrastructure and emergency management in New Zealand and further research on subseasonal-to-seasonal scale forecasting of AR events should remain a priority. In a warming climate, with the frequency and intensity of extreme events (ARs/floods/droughts) expected to increase, it is essential that we quantify the relative impacts of ARs. Future projections of AR frequency may then be interpreted in terms of impacts on local precipitation. Thus, the results presented in this chapter provide a useful baseline for assessing future climate change impacts. 


\section{Chapter 4}

\section{Large-Scale Drivers of ARs}

\subsection{Introduction}

Baroclinic eddies moving along mid-latitude storm tracks play a crucial role in the climate system, transporting moisture, sensible heat, and angular momentum poleward (Holton, 2004). Consequently, the position and strength of the storm track strongly influences precipitation and temperature variations across the mid-latitudes. Trenberth (1991) was the first to investigate Southern Hemisphere storm track characteristics based on zonally averaged meteorological variables. In the Southern Hemisphere, storm track activity is persistent year-round, with greatest activity near $50^{\circ} \mathrm{S}$ (Trenberth, 1991). There is significant seasonal variability in storm track activity, with zonal symmetry of the storm track greatest during summer, while storm track activity extends over a wider range of latitudes during winter (Trenberth, 1991). These seasonal changes are strongly linked to the position and intensity of the polar and subtropical jet streams (Trenberth, 1991). During austral summer, the lack of a developed subtropical jet results in the formation of a singular circumpolar storm track along the polar jet stream (Nakamura and Shimpo, 2004). During austral winter, the main upper-level storm track is located along the subtropical jet over the South Pacific, while a low-level storm track forms along the surface baroclinic zone off the coast of Antarctica (Nakamura and Shimpo, 2004). This results in a split-jet structure near New Zealand during winter, which acts to suppress storm track activity in the South Pacific.

During austral winter, the Southern Hemisphere split jet at the longitude of New Zealand increases AR frequency for northern regions, and reduces AR frequency for the South Island (Prince et al., 2021). Figure 4.1 shows the approximate locations of the mid-latitude storm track and jet-streams; we find that peak AR frequency occurs slightly equatorward of the location of maximum upper-level wind speed. The storm tracks emanate from regions of preferred cyclogenesis, where baroclinic instability of the mean flow, strong sea-surface temperature gradients, and strong upper-level divergence favour cyclone formation (Holton, 
2004). For the Southern Hemisphere, regions of preferred cyclogenesis are located off the eastern seaboards of South America and Australia, southeast of Africa, and near New Zealand (Sinclair, 1995). A number of studies have investigated the development and propagation of Southern Hemisphere synoptic-scale eddies, through tracking of cyclone and anticyclone centers (Lee and Held 1993; Berbery and Vera 1996; Chang 1999; Simmonds and Keay, 2000; Rao et al. 2002; Hoskins and Hodges, 2005). ARs provide yet another method to assess storm track activity. In particular, because the emphasis is on areas of high moisture transport (and individual high impact events) an AR climatology can reveal how the large-scale circulation influences the occurrence of extreme events (studies of other synoptic features may not resolve extremes to the same extent).

(a)

$\mathrm{DJF}$

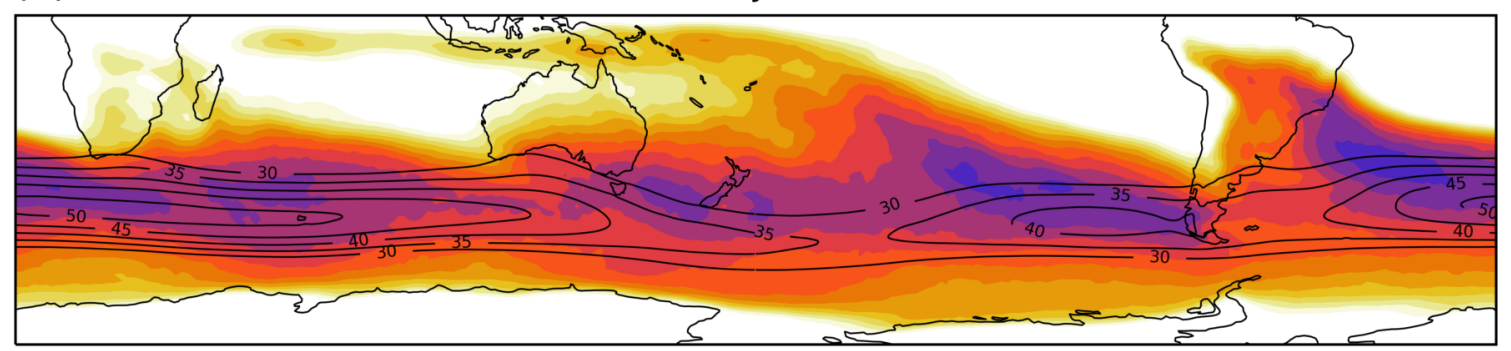

(b)

JJA
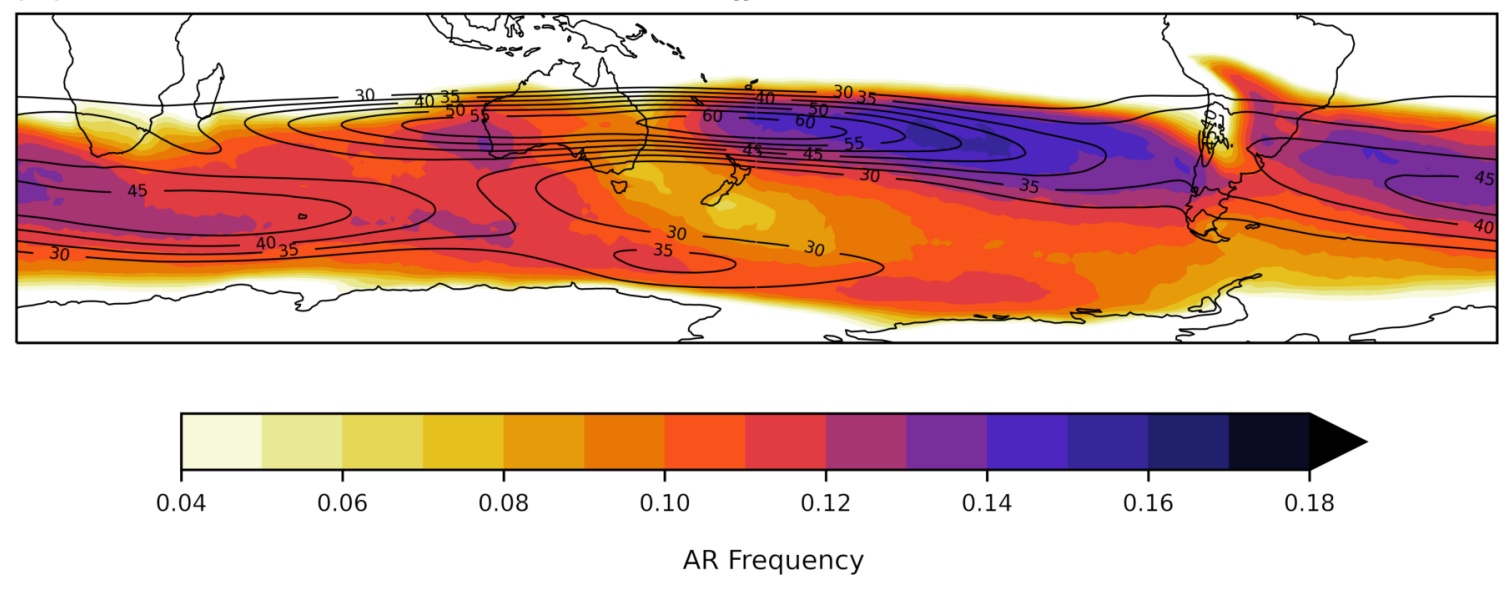

Figure 4.1: AR frequency and upper-level winds, showing approximate locations of the mid-latitude storm track and jet-streams. AR frequency (coloured contours) derived from global AR dataset of Guan \& Waliser (2015), and $300 \mathrm{hPa}$ wind speed (black contours; $\mathrm{ms}^{-1}$ ) derived from ERA5 reanalysis during (a) summer (DJF) and (b) winter (JJA), 1979-2019.

In a review of AR research, Gimeno et al. (2014) identified that "the connection between occurrence and activity of ARs and large-scale ocean-atmosphere dynamics is very poorly understood". While there have been a small number 
of investigations of large-scale climate modulation of ARs, the majority have focused on the west coast of North America and Europe. Guan et al. (2012) found that high impact AR landfalls in California were most frequent when the MJO is active over the western Pacific. Guan et al. (2013) further found that the negative phase of the Arctic Oscillation ${ }^{1}(\mathrm{AO})$, the Northern Hemisphere equivalent of the SAM, and Pacific-North American teleconnection pattern (PNA) were associated with more winter ARs in California. Studies of landfalling ARs in Western Europe found that the negative (positive) phase of the North Atlantic Oscillation (NAO) increased AR frequency over southern (northern) Europe (Lavers and Villarini, 2013). Making use of the global detection algorithm, Guan \& Waliser (2015) examined global AR frequency and AR precipitation anomalies associated with four prominent modes of large-scale climate variability for the boreal winter (November-March) period. The AO and PNA were investigated for the Northern Hemisphere, and the El Niño Southern Oscillation (ENSO) and Madden-Julian Oscillation (MJO) for both hemispheres. During its negative phase, the AO was found to significantly enhance AR frequency west of California, across the subtropical North Atlantic, and in western Greenland, and reduce AR frequency over Northern Europe. The magnitude of these anomalies were several times that of the seasonal cycle (Guan \& Waliser, 2015). El Niño conditions were found to strongly increase AR frequency in the northeastern Pacific and subtropical North Atlantic (Guan \& Waliser, 2015). For the Southern Hemisphere, ENSO was found to significantly modulate AR activity in the South Pacific convergence zone north of New Zealand, and the MJO was associated with increased AR activity between Australia and French Polynesia during phases 4-7 (see section 4.7). As the analysis was primarily focused on the Northern Hemisphere, and limited to the boreal winter (November-March) period, no specific impacts for New Zealand were highlighted.

Regional precipitation in New Zealand is strongly influenced by the patterns of large-scale atmospheric circulation, and the organized modes of climate variability operating on various spatio-temporal scales. These climate oscillations alter the storm track position and intensity as well as the mean pressure field across New Zealand, and influence the frequency of more structured objects such as ARs and atmospheric blocking highs. Previous investigations of New Zealand climate variables (e.g. precipitation, temperature, and soil moisture) have found significant correlations with the Southern Annular Mode (SAM), El-Niño Southern Oscillation (ENSO), the Interdecadal Pacific Oscillation (IPO), Indian Ocean Dipole (IOD), and the Madden-Julian Oscillation (MJ0) (Mullan, 1995; Salinger and Mullan, 1999; Salinger et al., 2001; Griffiths, 2007; Griffiths, 2011; Renwick, 2011; Fauchereau et al., 2016). While the impacts of these large-scale climate drivers in New Zealand is relatively well understood, there have been no formal

\footnotetext{
${ }^{1}$ The negative phase of the $\mathrm{AO}$ is associated with a more meridional jet structure that extends to lower latitudes.
} 
investigations of how they drive AR characteristics (including severity and duration) and AR frequency in New Zealand. It is important to quantify these impacts as ARs form an important subset of New Zealand weather systems; specifically, they are events associated with extreme impacts and flooding (see Chapter 3).

Subseasonal prediction refers to forecasts at lead times of around 1-5 weeks (7-45 days), filling the gap between medium-range and seasonal forecasts. Due to the appreciable socio-economic losses associated with ARs, skilful subseasonal forecasts of AR activity are of significant value. Subseasonal AR forecasts have potential applications in aiding disaster mitigation, drought/fire risk assessment, and agriculture management. However, there are considerable difficulties in forecasting at lead times of 1-5 weeks, often referred to as the 'predictability desert' (Vitart et al., 2012), as initial atmospheric conditions provide limited value at these timescales. However, large-scale climate oscillations can provide important sources of predictability at the subseasonal time-range. Thus, to improve subseasonal predictions of extreme weather, it is important to understand how large-scale oscillations influence AR behaviour.

For the Southern Hemisphere, where ARs have received relatively little formal study compared to the Northern Hemisphere, the connection between AR occurrence and large-scale ocean-atmosphere dynamics is poorly understood. In this chapter we provide the first investigation of how large-scale climate oscillations influence the occurrence of ARs. Again, we make use of Kidson types (which are known to be influenced by large-scale climate variability like ENSO and SAM) to quantify changes in the circulation patterns associated with ARs. As well as adding to the framework of AR research in the understudied southwest pacific region, our investigation furthers the global understanding of AR variability. These results may have important implications for subseasonal-to-seasonal scale forecasting in New Zealand.

\subsection{Data and Methods}

To assess the impacts of climate variability on landfalling ARs in New Zealand, we use two AR datasets, complemented by a daily rainfall dataset comprising 189 stations over the 1979-2019 period. In previous chapters we found a distinct seasonality in AR frequency, which also differed significantly between North and South Islands. This was mostly due to seasonal changes in the position of the jet stream near New Zealand (see Figure 4.1). As the SAM and ENSO significantly influence the strength and position of the mid-latitude jet stream, there exists a complex interaction between these patterns of climate variability and the seasonal cycle. The respective influence of each climate mode also varies with latitude, and is highly regional (Ummenhofer and England, 2007), therefore we expect impacts on AR frequency to vary significantly by region. Climate variabil- 
ity in the North Island is more linked to the tropical pacific (and ENSO), and sea surface temperature (SST) anomalies in the southern Tasman Sea (Ummenhofer and England, 2007). Climate variability in the South Island is mostly linked to the large-scale atmospheric circulation, namely, the strength and position of the Southern Hemisphere westerly wind belt (i.e. the SAM phase) (Ummenhofer and England, 2007). To account for the seasonal cycle, and regional differences, we calculate anomalies separately for each season and region. We calculate monthly anomalies of AR count by region, including counts of 'heavy' (daily precip. above 99th percentile) and 'extreme' (daily precip. above 100mm) ARs. We also calculate monthly anomalies of the number of each Kidson type concurrent with each $\mathrm{AR}$, to investigate changes in the circulation patterns driving ARs. Anomalies in AR characteristics (such as mean IVT magnitude and duration) were also investigated.

The analysis in this chapter is based on the New Zealand AR dataset (except where mentioned otherwise, in which case we use the global AR dataset of Guan \& Waliser (2015)). The New Zealand AR dataset (Kennett, 2020) is derived using a higher IVT threshold compared to Guan \& Waliser (2015) to focus on the strongest events, and operates at higher spatial resolution to better resolve regional differences. From the monthly anomalies, we calculate composite means of AR counts for each season and climate mode phase. For composite analysis, the phases of each climate oscillation are defined using their respective indices. Positive (negative) phases are defined as months where the index is greater than (less than) 0.5 standard deviations of the full climatology (1979-2019). Neutral phase occurs where the index is within 0.5 standard deviations. Statistical significance is based on a two-sided t-test for the null hypothesis that the mean of the sample of observations is equal to zero. We highlight anomalies significant at the $90 \%$ level (i.e with a probability p-value $<0.1$ ) for AR frequency maps, and the $85 \%$ and $95 \%$ confidence levels for monthly count anomalies. It is also worth noting that as monthly AR frequency is based on a binary index (i.e. 1 if an AR is detected, and 0 otherwise), statistically significant results are not always pronounced. The most significant AR frequency anomalies are often seen to the north and south of New Zealand. However, even for these cases, significant increases and decreases in AR activity can certainly impact New Zealand rainfall as AR impacts typically extend outside of the boundaries of the AR.

We are particularly interested in how various climate modes influence the occurrence of extreme events. Again, we classify the most intense ARs in terms of daily precipitation. If at least one station records a daily rainfall total above the 99th percentile (defined relative to all daily rainfall observations for that station), either the day of or day after AR landfall, that AR is labeled as a 'Heavy' AR. If at least one station records a daily rainfall total above $100 \mathrm{~mm}$, either the day of or day after AR landfall, that AR is labeled as an 'Extreme' AR. Again, to assess regional impacts, we use the six regional climate zones defined by Mullan (1998). 
See Figure 3.3 for a map of the region boundaries. The 12 Kidson types outlined in Chapter 2 are used to understand the synoptic conditions associated with each AR (see Figure 2.11 for plots of the mean $1000 \mathrm{hPa}$ geopotential height associated with each Kidson type). Using the Kidson type framework we may associate each AR with a simple regional circulation pattern, and investigate changes in the favoured AR circulation patterns for each climate mode.

For comparison, the raw monthly mean AR counts for each season and region are presented below. Note that AR frequency anomalies in the range $1-5 \%$ are not trivial, considering the mean AR frequency over New Zealand is only approximately $6 \%$. Thus, even an increase or decrease in AR frequency of $0.5 \%$ equates to a change of almost $10 \%$.

Table 4.1: New Zealand monthly mean AR counts by season and region.

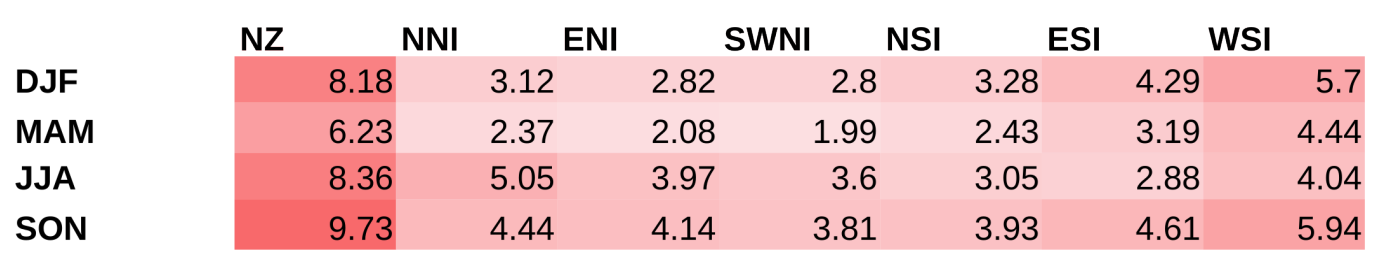

Table 4.2: New Zealand monthly mean 'Heavy' AR counts by season and region.

\begin{tabular}{cccccccc} 
& NZ & NNI & ENI & SWNI & NSI & ESI & WSI \\
\hline DJF & 5.62 & 2.02 & 1.63 & 2.08 & 1.82 & 3 & 3.82 \\
\hline MAM & 4.87 & 1.95 & 1.7 & 1.97 & 1.79 & 2.26 & 2.93 \\
JJA & 6.97 & 3.78 & 3.35 & 3.88 & 2.97 & 3.52 & 2.93 \\
SON & 6.86 & 2.54 & 2.79 & 3.21 & 2.6 & 3.75 & 3.58
\end{tabular}

Table 4.3: New Zealand monthly mean 'Extreme' AR counts by season and region.

\begin{tabular}{c|ccccccc} 
& NZ & NNI & ENI & SWNI & NSI & ESI & WSI \\
\hline DJF & 2.15 & 0.31 & 0.09 & 0.18 & 0.25 & 0.82 & 1.55 \\
MAM & 1.65 & 0.18 & 0.09 & 0.15 & 0.18 & 0.67 & 1.18 \\
JJA & 2.67 & 0.75 & 0.24 & 0.24 & 0.29 & 1.14 & 1.55 \\
SON & 2.38 & 0.31 & 0.14 & 0.19 & 0.27 & 1.29 & 1.83
\end{tabular}


Table 4.4: New Zealand monthly mean AR Kidson Type counts by season.

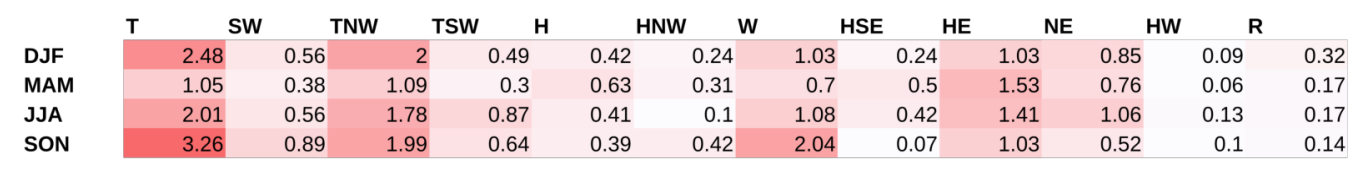

\subsection{SAM}

The Southern Annular Mode (SAM) (also known as the Antarctic Oscillation or $\mathrm{AAO}$ ) is the leading mode of climate variability in the extratropical (poleward of $20^{\circ} \mathrm{S}$ ) Southern Hemisphere (Thompson and Wallace, 2000). The SAM phase describes pressure anomalies over Antarctica and the middle latitudes, which tend to be of opposite sign and fluctuate together on monthly time scales (e.g. negative pressure anomalies over Antarctica are associated with positive pressure anomalies in the middle latitudes, termed the positive phase of the SAM; with opposite sign pressure anomalies for the negative phase of the SAM). This seesaw in pressure alters the north-south pressure gradient over the mid-latitudes thereby influencing the position and intensity of the mid-latitude westerly wind belt, and determines the strength and location of baroclinic systems across the New Zealand region. A positive (negative) SAM phase is associated with a poleward (equatorward) shift in the storm track and jet stream. The SAM shows significant seasonal variation, with departures from a symmetric 'annular' structure associated with the split jet near New Zealand during austral winter and spring (Fogt et al., 2012). On weekly time-scales, the SAM oscillates unpredictably between phases, though on monthly to seasonal time-scales variations are driven by tropical SSTs (especially those associated with ENSO) and stratospheric ozone concentrations over Antarctica (Gillett and Thompson, 2003; Lim et al., 2013).

Figure 4.2 shows the seasonal IVT and MSLP anomalies associated with the positive SAM phase. Surface pressures are higher over New Zealand, especially southeast of the South Island, resulting in weaker westerly winds and settled weather over the country. In general a strengthening of the IVT magnitude south of $50^{\circ} \mathrm{S}$ is observed for all seasons, associated with stronger westerly flow. Over New Zealand, northwesterly IVT anomalies are observed (with a significant reduction in IVT magnitude). IVT magnitude is most strongly modulated during the warmer months, when the atmospheric water vapour content throughout the southern mid-latitudes is much higher. Weaker IVT anomalies are observed over New Zealand during winter, and IVT anomalies are more meridional compared to summer.

Mostly opposite patterns occur during the negative SAM phase; surface pressures 
(a)

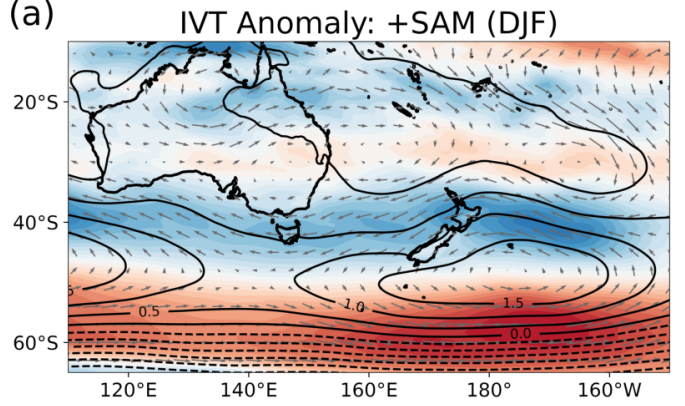

(c) IVT Anomaly: + SAM (JJA)

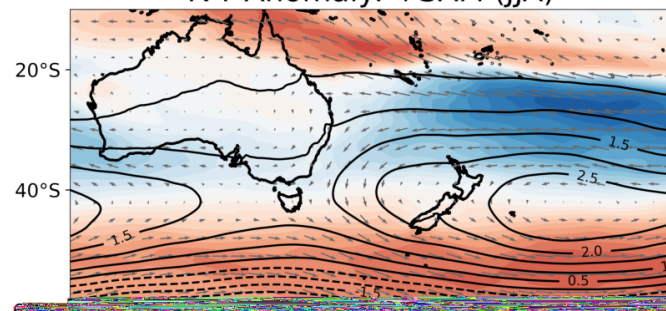

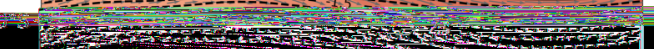
$1.70 \%$ : D. $100^{\circ}$

30 (b) IVT Anomaly: +SAM (MAM)

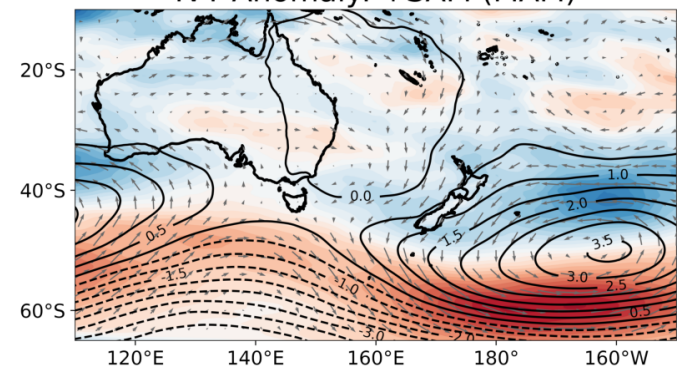

(d)

IVT Anomaly: +SAM (SON)

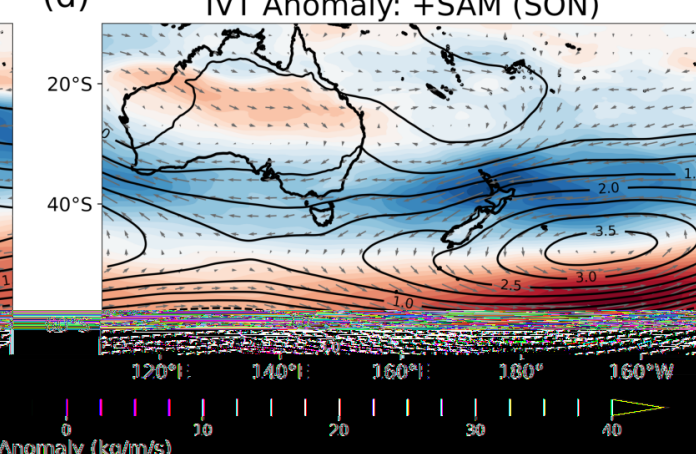

Figure 4.2: Composite IVT and MSLP anomalies for positive SAM months for each season (1979-2019). Seasonal IVT anomalies (coloured contours and vectors), and MSLP anomalies (black contours), derived from ERA5 reanalysis. 
are lower over New Zealand, especially southeast of the South Island, resulting in considerably stronger westerly winds over the country (especially in spring and summer). Over New Zealand, strong southwesterly IVT anomalies are observed, with significant increases in IVT magnitude over the North Island (Figure 4.3).
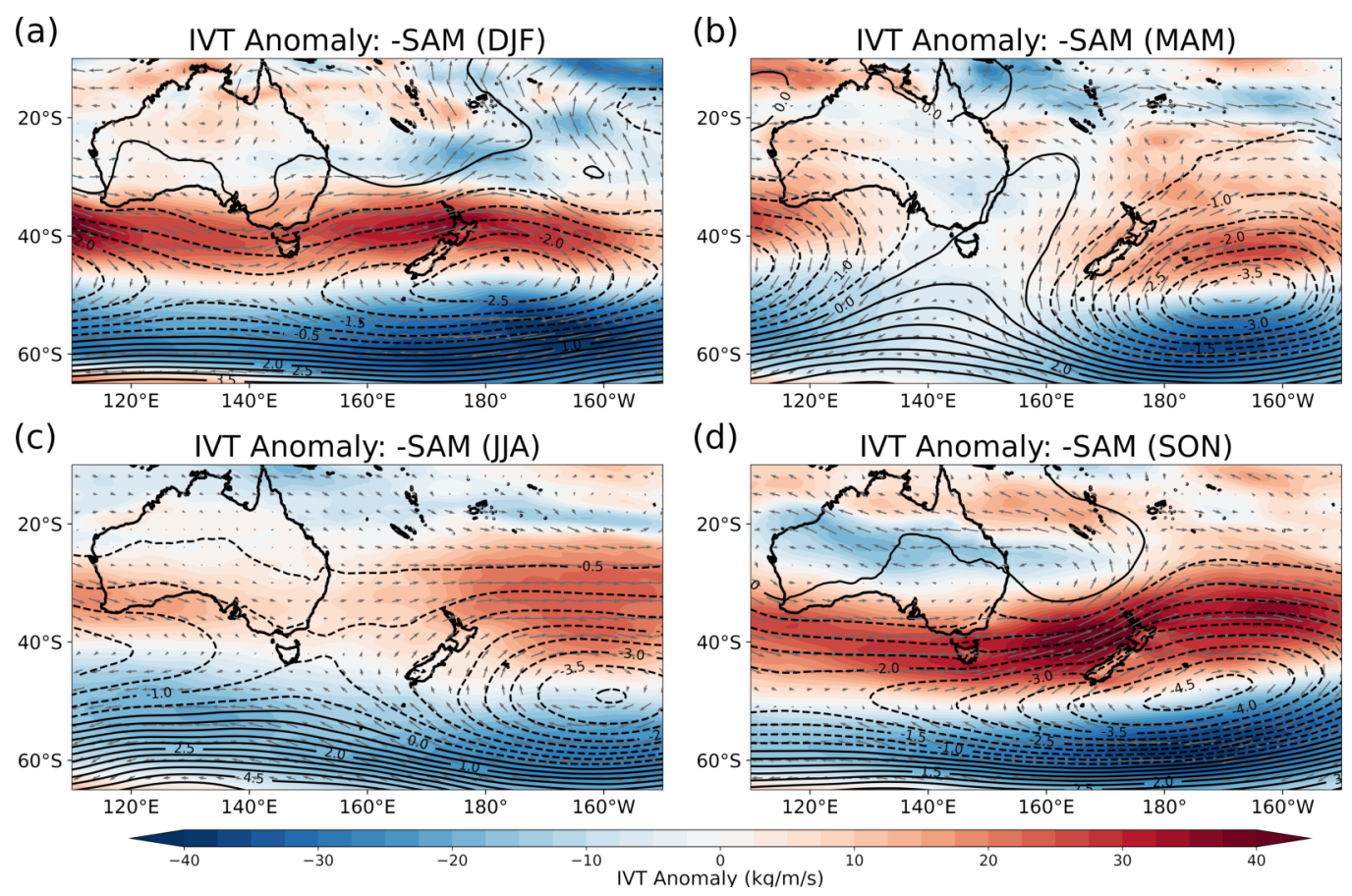

Figure 4.3: Composite IVT and MSLP anomalies for negative SAM months for each season (1979-2019). Seasonal IVT anomalies (coloured contours and vectors), and MSLP anomalies (black contours), derived from ERA5 reanalysis.

As the SAM is related to the strength of the zonal flow over New Zealand, the SAM phase has a significant impact on New Zealand climate (particularly for orographic precipitation on the west coast). The positive SAM phase is associated with a reduction in the strength of the prevailing westerly flow, and a poleward shift of the storm track away from New Zealand, and increase in easterly flow. This results in less orographic precipitation generation over New Zealand, reducing rainfall and increasing temperatures for western parts of the North and South Islands (Renwick and Thompson, 2006; Kidston et al., 2009). The opposite is true for the negative phase of the SAM, during which the storm track shifts equatorward over New Zealand resulting in an increase in moist, westerly airflow. The spatial structure of the SAM shows significant seasonal variations. For the New Zealand region, this seasonality results in more zonal wind speed anomalies dur- 
ing summer and more meridional wind speed anomalies during winter (Kidston et al., 2009). The SAM has also been shown to modulate the frequency of certain Kidson types. In general, more 'trough' types are observed during the negative SAM phase, while more 'zonal' and 'blocking' types are observed during the positive SAM phase (Renwick, 2011). Over the last 60 years, there has been a positive trend in the SAM index, and an associated strengthening and poleward contraction of the circumpolar westerlies. This change is mainly attributed to depletion of ozone in the lower stratosphere over Antarctica (Thompson et al., 2011). This change is mainly attributed to depletion of ozone in the lower stratosphere over Antarctica during austral spring (Gillett and Thompson, 2003; Thompson et al., 2011). Thus, knowledge of how the SAM influences ARs can give insight into how ozone recovery and increasing greenhouse gas concentrations will influence New Zealand rainfall, drought, and flood risk.

For our analysis, the NOAA SAM index is used to represent the SAM. The index is derived using principal component analysis, based on data from the NCEP/NCAR reanalysis dataset. The index is constructed by projecting the monthly $700 \mathrm{hPa}$ geopotential height anomalies south of $20^{\circ} \mathrm{S}$ onto the 'loading pattern of the AAO' ${ }^{\prime 2}$. See Thompson and Wallace (2000) for a full description of the calculation method. A number of other SAM indices exist, either based on principal component analysis or the difference in zonal mean pressure between $40^{\circ} \mathrm{S}$ and $65^{\circ} \mathrm{S}$. The indices may be based on station records or reanalysis products. The advantage of indices based on station records (such as the Marshall (2003) index) is they allow for a SAM index to be derived back to 1957 from available station data. Reanalysis data over the Southern Hemisphere (for principal component analysis) are only reliable during the modern satellite era which began in 1979. Since our AR study period (which relies on reanalysis data) begins in 1979, we use the NOAA SAM index. This index was obtained from the NOAA Climate Prediction Center (CPC), and can be accessed at: https://www. cpc.ncep.noaa. gov/products/precip/CWlink/daily_ao_index/aao/aao.shtml.

\section{SAM AR Anomalies}

We find the SAM to be the dominant driver of AR frequency in New Zealand, though the effects vary by season and location. Figure 4.5 shows the seasonal AR frequency anomalies associated with the positive SAM phase. AR frequency south of New Zealand is strongly modulated by the phase of the SAM; significantly more ARs are observed between $50-60^{\circ} \mathrm{S}$ for all seasons during the positive phase. This increase in AR frequency south of New Zealand results in more AR landfalls for the South Island during winter and spring, and for the southern

\footnotetext{
${ }^{2}$ The loading pattern of the AAO is defined as the leading Empirical Orthogonal Function (EOF) analysis of monthly mean $700 \mathrm{hPa}$ height anomalies during the 1979-2000 period.
} 


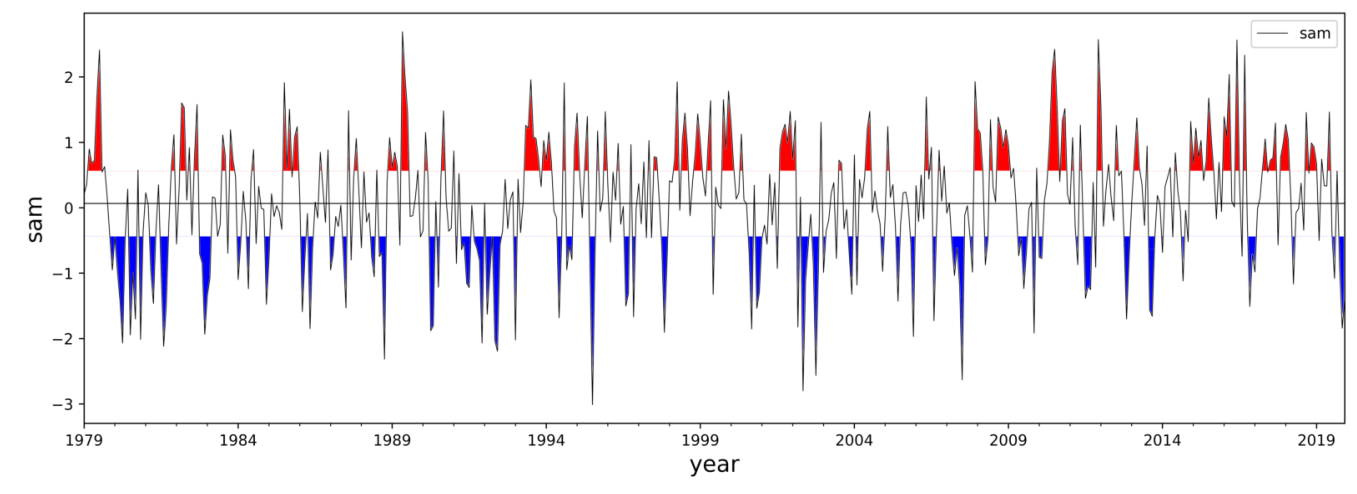

Figure 4.4: Monthly SAM index from 1979-2019. Values +/- 0.5 standard deviation are coloured.

South Island for all seasons. In particular, the western South Island receives $25 \%$ more landfalling ARs during winter (statistically significant at the $95 \%$ level) ${ }^{3}$. While AR frequency increases for the very south of the country, overall the number of landfalling ARs in New Zealand during summer decreases by $16 \%$ as the storm-track shifts poleward. Significantly fewer ARs are observed over the North Island during summer ( $30-35 \%$ fewer), and the northeast North Island during winter and spring. The fraction of ARs contributing to daily rainfall totals above the 99th percentile decreases for the northern North Island and eastern South Island during summer, and for the eastern North Island during winter and spring. ARs are associated with more $\mathrm{H}$ and HE Kidson types, and fewer T, SW, TSW, HNW and W Kidson types. Thus, during positive SAM, more ARs are driven by synoptic types associated with strong airflow on the southern periphery of highpressure systems (making landfall in the southern South Island). Fewer ARs are associated with lower latitude troughs (i.e. those types featuring strong westerly airflow over the North Island), due to the poleward shift of the storm-track.

During a negative SAM phase, AR frequency increases across all regions of New Zealand for spring and summer as stronger westerlies and increased atmospheric moisture drive positive IVT anomalies. During autumn and winter, negative SAM is associated with a decrease in AR frequency for central and southern New Zealand, including a decrease in the number of 'heavy' and 'extreme' events ${ }^{4}$. This coincides with a decrease in the number of TSW, H, and HE Kidson types. There is also an increase in AR frequency for the northern North Island in winter. ARs are associated with more T, SW, and W Kidson types (i.e. those types featuring strong westerly winds over the North Island), and fewer HE and NE Kidson types. The SAM modulates AR frequency most strongly during spring and summer, when the IVT magnitude over New Zealand is higher. Changes in

\footnotetext{
${ }^{3}$ See Appendix B

${ }^{4}$ See Appendix B
} 
AR frequency appear to be due to changes in the circulation patterns favourable for AR landfalls, with more ARs associated with 'trough' types during the negative SAM phase, and more ARs associated with the 'blocking' regime during the positive SAM phase. We did not find any statistically significant changes in IVT magnitude due to the SAM phase. This is expected, as variability in the SAM is associated with changes in cyclone track frequency, but not cyclone intensity (Eichler and Gottschalck, 2013). Overall, aside from the seasonal cycle, the SAM is the dominant source of variability in AR frequency in New Zealand. 
(a)

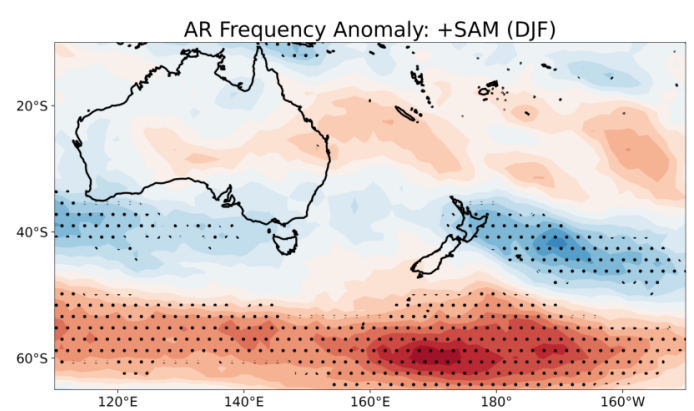

(c)

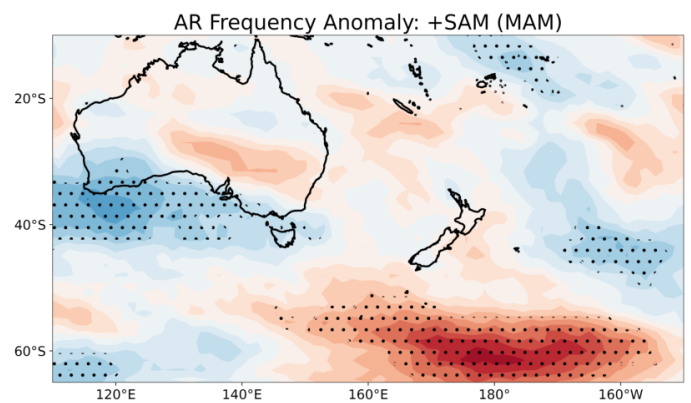

(e)

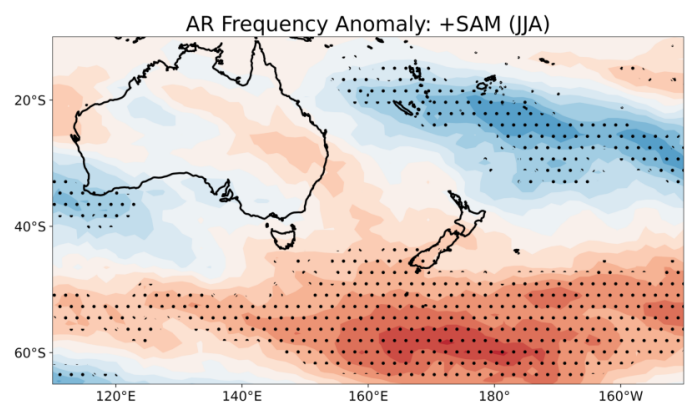

(g)

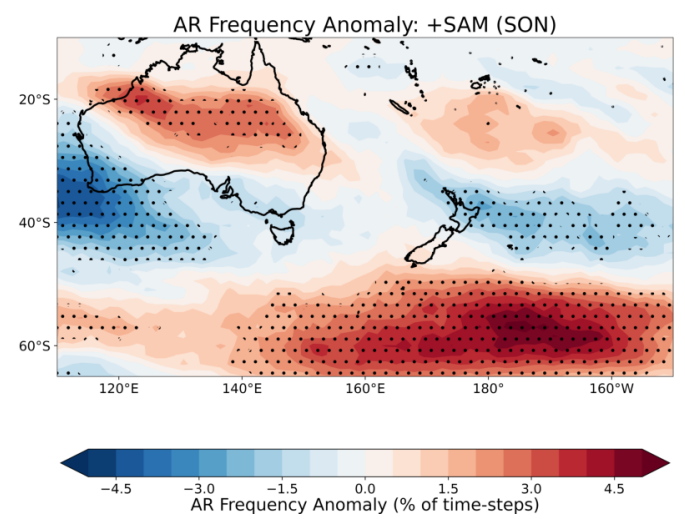

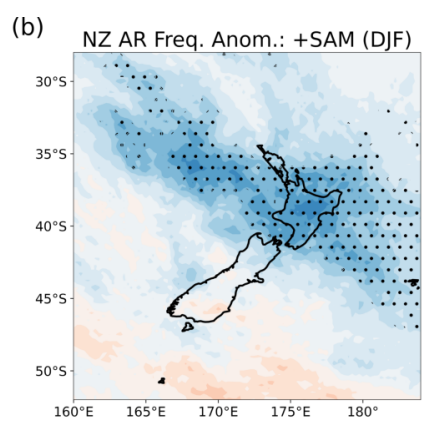

(d)

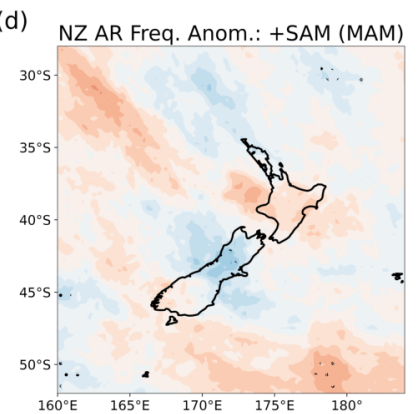

(f)

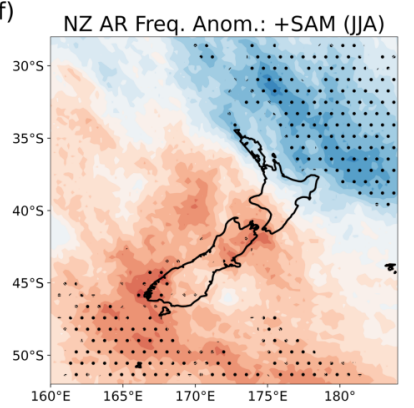

(h)

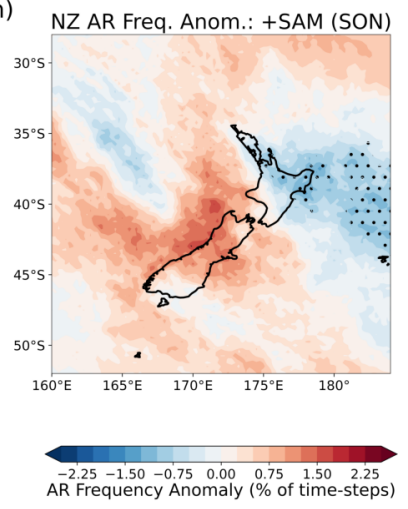

Figure 4.5: Composite AR frequency anomaly for positive SAM months for each season (1979-2019). (a, c, e, g) are derived from the global AR dataset (Guan \& Waliser, 2015), while (b, d, f, h) are derived from the NZ AR dataset. Dotted areas denote anomalies significant at the $90 \%$ level. 
(a)

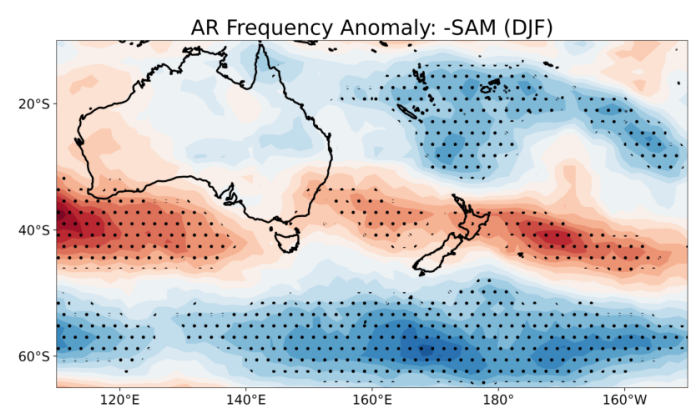

(c)

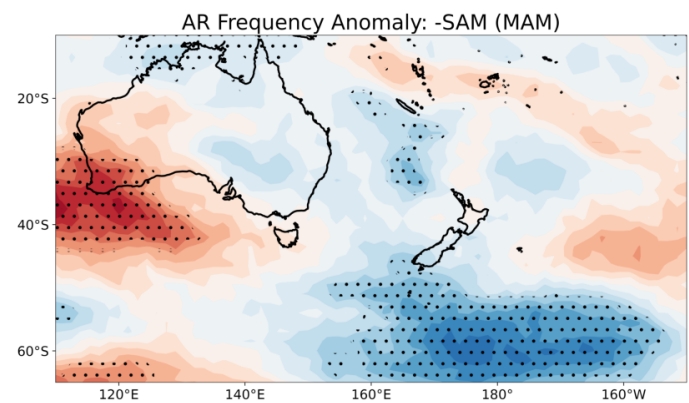

(e)

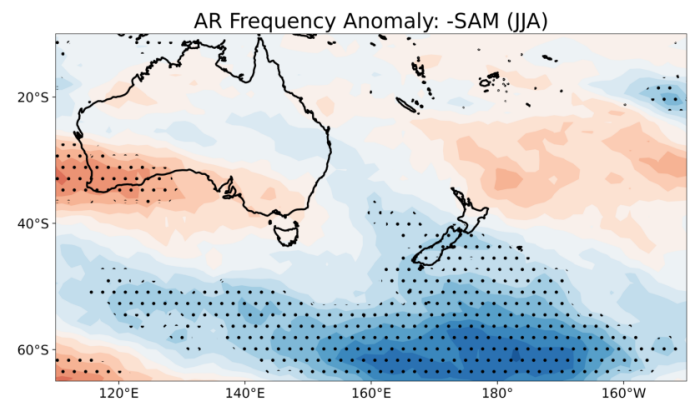

(g)

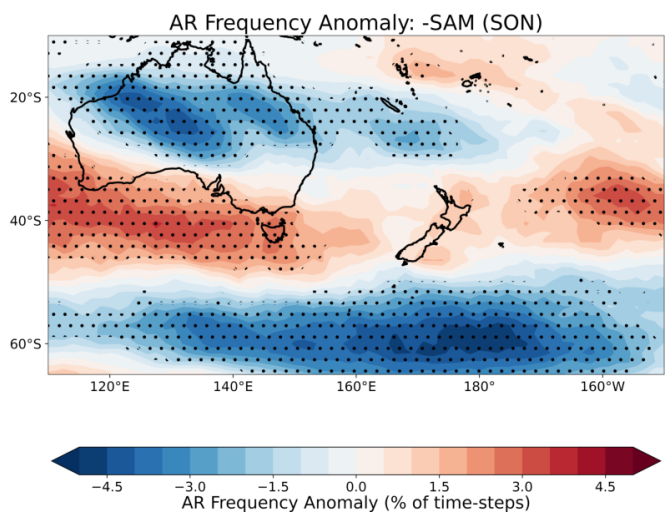

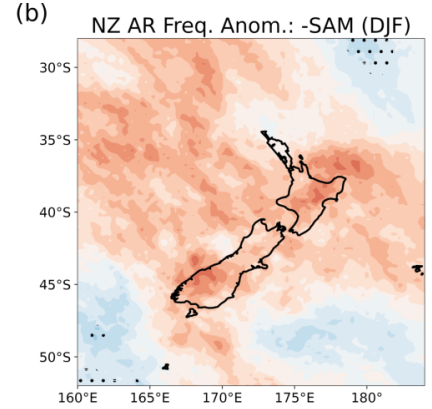

(d) NZ AR Freq. Anom.: -SAM (MAM)

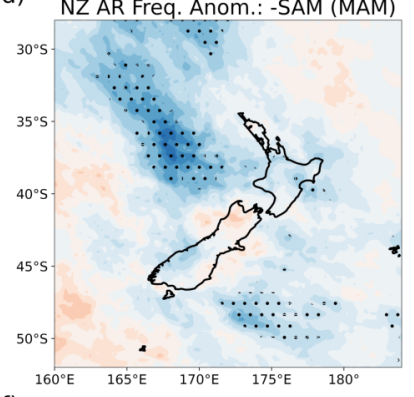

(f)

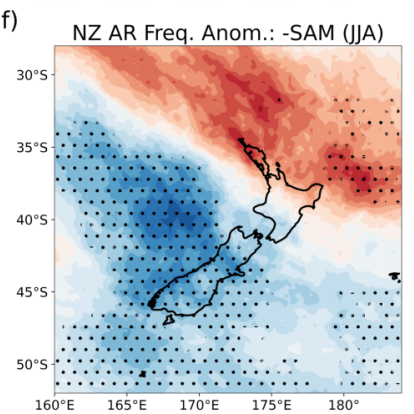

(h)

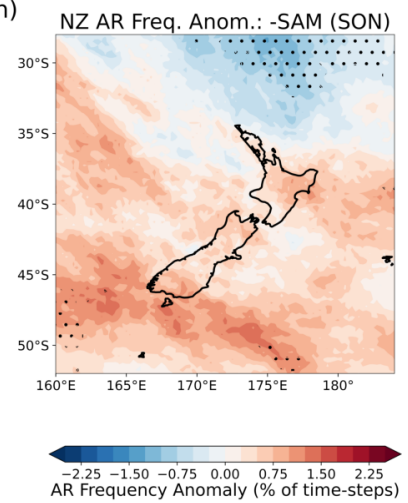

Figure 4.6: Composite AR frequency anomaly for negative SAM months for each season (1979-2019). (a, c, e, g) are derived from the global AR dataset (Guan \& Waliser, 2015), while (b, d, f, h) are derived from the NZ AR dataset. Dotted areas denote anomalies significant at the $90 \%$ level. 


\section{$4.4 \quad$ ENSO}

The El Niño-Southern Oscillation (ENSO) is a recurrent, quasi-periodic phenomenon, describing fluctuations in ocean surface temperatures and atmospheric circulation in the equatorial Pacific (see for e.g. Bjerknes, 1966). Across the tropical Pacific, the permanent and prevailing 'trade winds' blow from east to west, pushing warm surface water to the western Pacific near Asia and Australasia. As the warmer water is pushed away, it is replaced by upwelling of cold water in the eastern Pacific near South America. Over the warmer western Pacific waters, enhanced rainfall and convection creates an area of lower surface pressure compared to the eastern Pacific. The resulting temperature/pressure gradient induces a circulation pattern across the tropical Pacific, known as the Walker circulation, with warm moist air rising in the west and cooler dry air descending in the east. Naturally occurring variations in the sea surface temperatures and pressure in the central and eastern tropical Pacific (influencing the Walker circulation) gives rise to the ENSO phenomenon. During the El Niño phase, tropical sea surface temperatures off the Pacific coast of South America are anomalously warm and atmospheric pressure is anomalously low. This weakens the SST/pressure gradient across the tropical Pacific, and causes the trade winds to weaken (or even reverse). During the La Niña phase, tropical sea surface temperatures off the Pacific coast of South America are anomalously cool and atmospheric pressure is anomalously high. This strengthens the SST/pressure gradient across the tropical Pacific, and causes the trade winds to strengthen. El Niño/La Niña events typically occur once every two to seven years, though in an irregular and unpredictable cycle (McPhaden et al., 2006). Typically ENSO is strongest during the austral spring and summer, though can occur year-round (Mullan and Thompson, 2006). Recent development of coupled ocean-atmosphere models has allowed ENSO to become the most predictable climate mode on monthly to seasonal time scales (Tang et al., 2018). El Niño and La Niña events can be forecast 6-12 months ahead of time, and tend to follow predictable patterns once they have commenced (Tang et al., 2018). Thus, understanding how ENSO modulates AR activity is important for long-range predictability of heavy rainfall events.

Figure 4.7 and Figure 4.8 show the seasonal IVT and MSLP anomalies associated with El Niño and La Niña phases respectively. Note the considerable IVT anomalies in the tropics during autumn and winter, driven by a strengthening of the trade winds. During El Niño events, the subtropical jet strengthens, enhancing the subtropical/polar jet bifurcation and reducing storm track activity over the South Pacific (Ashok et al., 2007). In general, IVT is higher in magnitude and anomalously southwesterly across New Zealand during El Niño events for all seasons.

The changes in atmospheric circulation associated with ENSO can have signifi- 
(a)

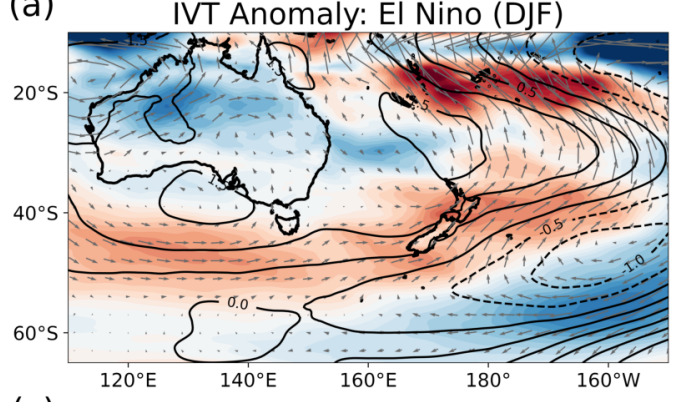

(c) IVT Anomaly: El Nino (JJA)

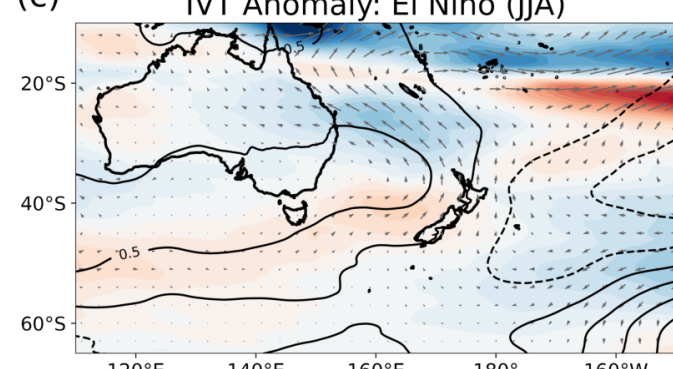

$\overbrace{-40}^{20^{\circ} \mathrm{E}}$ (b)

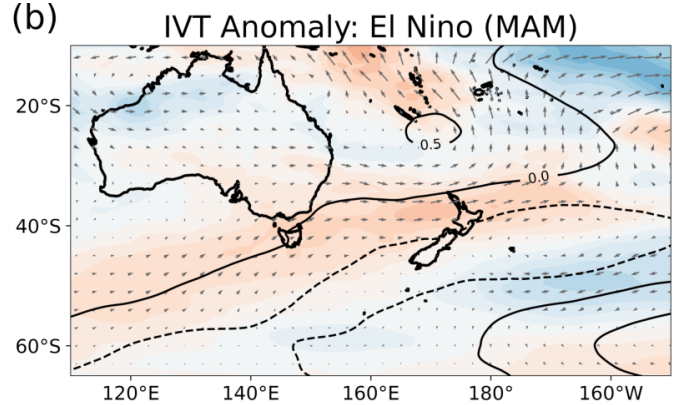

(d)

IVT Anomaly: El Nino (SON)

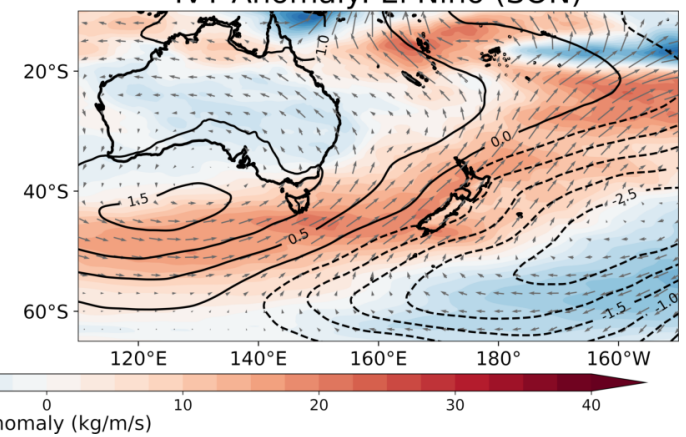

Figure 4.7: Composite IVT and MSLP anomalies for El Niño months for each season (1979-2019). Seasonal IVT anomalies (coloured contours and vectors), and MSLP anomalies (black contours), derived from ERA5 reanalysis. 
(a)

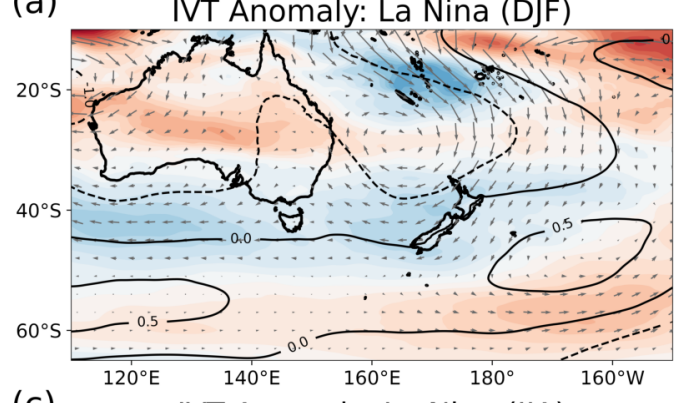

(c)

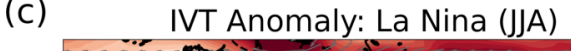

$20^{\circ} \mathrm{s}$

$40^{\circ} \mathrm{S}$

$60^{\circ} \mathrm{S}$

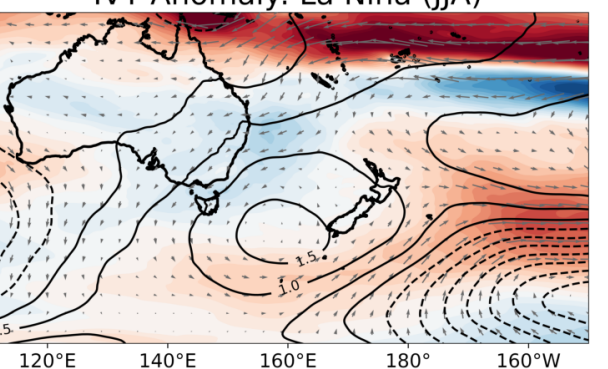

(b) IVT Anomaly: La Nina (MAM)

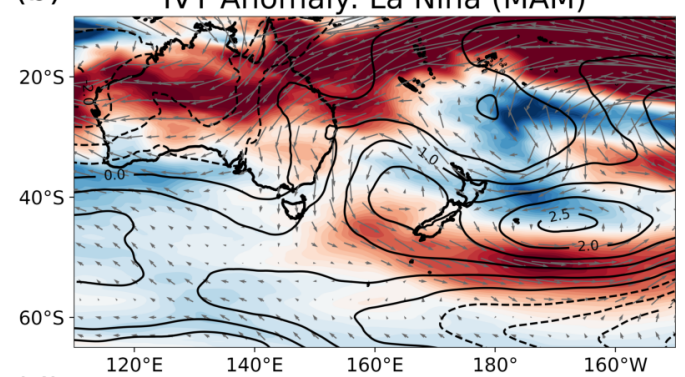

(d)

IVT Anomaly: La Nina (SON)

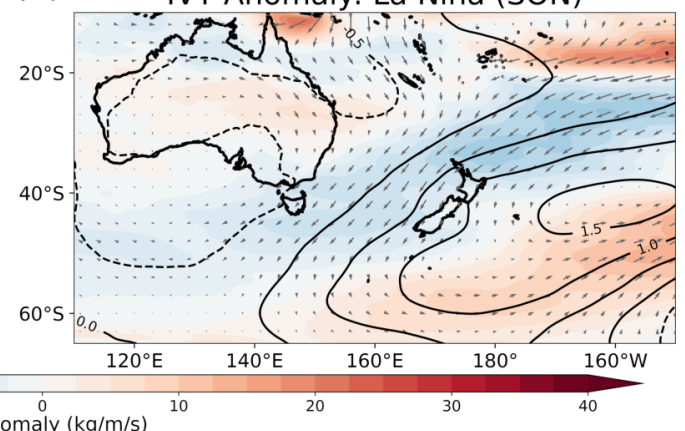

Figure 4.8: Composite IVT and MSLP anomalies for La Niña months for each season (1979-2019). Seasonal IVT anomalies (coloured contours and vectors), and MSLP anomalies (black contours), derived from ERA5 reanalysis. 
cant global and regional impacts. ENSO events influence storm track activity in the Southern Hemisphere by altering the position and intensity of the subtropical and polar jet streams. Convective activity in the tropics influences the subtropical jet through anomalous divergent winds, and the polar jet through generation of stationary Rossby waves (Sardeshmukh and Hoskins, 1985; Kidson and Renwick, 2002; Nakamura and Shimpo, 2004). Typically, El Niño events increase rainfall across the Americas and induce droughts across Southeast Asia and Australia (with opposite impacts during La Niña events). The 1997-98 El Niño event, one of the strongest on record, was associated with drought conditions throughout Southeast Asia, severe flooding in Peru, increased rainfall in California and record-high temperatures across the American Midwest (McPhaden, 1999). ENSO also strongly impacts Earth's polar regions (through propagation of Rossby waves generated by tropical convection), with recognized impacts on sea and glacial ice (Turner, 2004; Clem et al., 2016; Nicolas et al., 2017; Yuan et al., 2018). As New Zealand is situated far to the south of the equator, and between the rising and sinking branches of the Walker circulation, the impact of ENSO on the mean pressure gradient over New Zealand is small but notable. In general, the impacts of El Niño/La Niña events in New Zealand are variable and seasonal. Further, the impacts of La Niña are not always equal and opposite to El Niño impacts (Mullan, 1995).

Previous research has found El Niño phases are associated with stronger and more frequent westerly/southwesterly winds, and therefore increased rainfall for the west of the country and reduced precipitation in the east (Gordon, 1986; Mullan, 1995; Salinger and Mullan, 1999; Griffiths, 2007). Therefore El Niño has a similar impact on NZ climate as the negative phase of the SAM, though its impact is more variable than the SAM. La Niña phases were associated with stronger and more frequent northeasterly winds, and therefore reduced precipitation in the west (Gordon, 1986; Mullan, 1995; Salinger and Mullan, 1999; Griffiths, 2007), similar to the positive phase of the SAM. Overall, ENSO influences almost $25 \%$ of the year-to-year variability in New Zealand rainfall and temperatures (Griffiths, 2011). ENSO has significant influence on river flows in New Zealand, with North Island rivers experiencing increased (reduced) flood peaks during La Niña (El Niño) events (Mosley, 2000; Scarsbrook et al., 2003). Kidson and Renwick (2002) investigated how characteristic patterns of tropical convection (based on cluster analysis of outgoing longwave radiation satellite observations), influence New Zealand weather. During El Niño conditions, southwest flow prevails over New Zealand. During 'strong' El Niño conditions (where the centre of convection is displaced further east), stronger west-southwest anomalies are observed over New Zealand. El Niño conditions were associated with stronger southerly flow anomalies, increased frequency of 'zonal' Kidson type regimes, and decreased frequency of 'blocking' Kidson regimes (where the anomalies are greater for 'strong' El Niño conditions). La Niña conditions were associated with northerly flow anomalies, reduced frequency of 'zonal' Kidson type regimes, and 
increased frequency of 'blocking' Kidson regimes.

For our analysis, the Eastern Tropical Pacific SST (Niño 3.4) index is used as a proxy for the El Niño-Southern Oscillation (ENSO). The index is based on the sea surface temperature (SST) anomalies over the region bounded by $5^{\circ} \mathrm{N}-5^{\circ} \mathrm{N}$ and $170^{\circ} \mathrm{W}-120^{\circ} \mathrm{W}$, and is among the most widely used ENSO indices. A number of other ENSO indices exist, usually based on SST anomalies averaged over different tropical Pacific regions (e.g. Niño 1+2, Niño 3, Niño 4, ONI and TNI), or atmospheric pressure anomalies (e.g. SOI, Equatorial SOI). This index was obtained from the NOAA Climate Prediction Center (CPC).

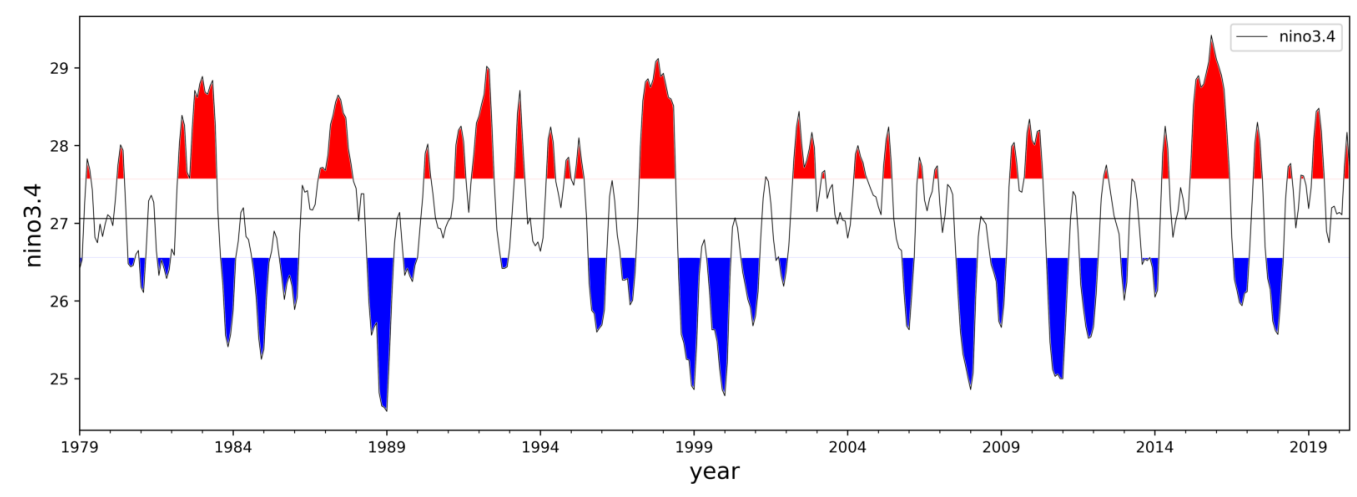

Figure 4.9: Monthly Niño 3.4 index from 1979-2019. Values +/- 0.5 standard deviation are coloured.

\section{ENSO AR Anomalies}

As expected for a tropical climate driver, the most significant AR frequency anomalies are found in the northern North Island (and are strongest during summer when the ENSO magnitude peaks). Although the AR frequency patterns are not always clear, for El Niño months we tend to observe lower AR frequency anomalies for northern New Zealand and increased AR frequency for southwestern New Zealand. Over New Zealand as a whole, more ARs are observed overall due to the increased frequency of West Coast ARs. There is also a statistically significant decrease in the mean IVT of ARs. The frequency of ARs contributing to 'Heavy' rainfalls (>99th percentile) in the North Island and northern South Island is reduced during El Niño events (statistically significant at the $95 \%$ level) ${ }^{5}$. ARs are associated with more SW, HNW and HE Kidson types, and fewer TSW and NE Kidson types.

La Niña is associated with increased AR frequency to the northeast and south

\footnotetext{
${ }^{5}$ See Appendix B
} 
of New Zealand during summer and autumn. In general, La Niña is associated with an increase in the number of 'Heavy' AR events during winter and spring. During autumn, significantly fewer ARs are observed in the southwestern North Island, northern South Island and eastern South Island, and ARs are associated with more H, HSE and HE Kidson types, and fewer SW, TSW, HNW, W and NE Kidson types. There is also a statistically significant increase in the mean IVT of ARs and the IVT magnitude at landfall for La Niña months. Thus, although ENSO does not significantly influence AR frequency, it does influence AR characteristics. These patterns appear to be consistent with the general circulation under ENSO, and the existing literature on ENSO and New Zealand rainfall.

In our climatology of New Zealand ARs, we find that a significant number of ARs are associated with ex-tropical cyclones. Previous research has linked tropical cyclone activity in the southwest Pacific to ENSO phases (Sinclair, 2002; Diamond et al., 2013). However, Lorrey et al. (2014) found that the location of ex-tropical cyclones affecting northern New Zealand were not directly linked to the phase of ENSO or SAM. These events were instead driven by "(1) weak blocking in the southwest Pacific that allows ex-tropical cyclones to enter the New Zealand sector and (2) prevalent atmospheric circulation patterns within the south Pacific sector that (a) guide the storms into the extratropical transition region north of the country which are then (b) pushed either to the west or east of the North Island due to distinct Kidson (2000) synoptic type occurrences" (Lorrey et al., 2014). Therefore it is likely that regional circulation patterns, rather than the ENSO or SAM phase, are more important in determining whether ARs associated with ex-tropical cyclone make landfall in New Zealand. Indeed, we did not find any relationships between the ENSO phase and the frequency of 'tropical' ARs. 
(a)

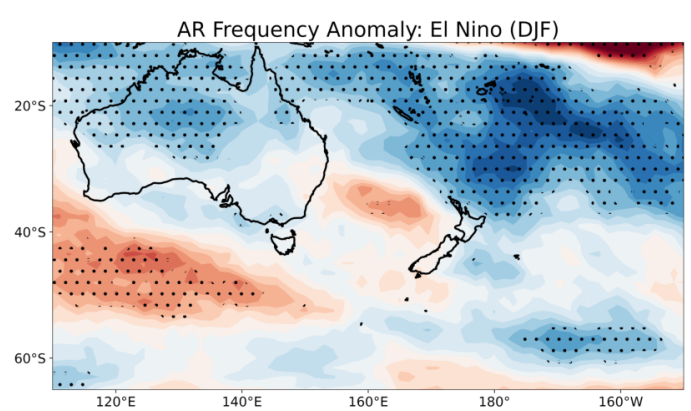

(c)

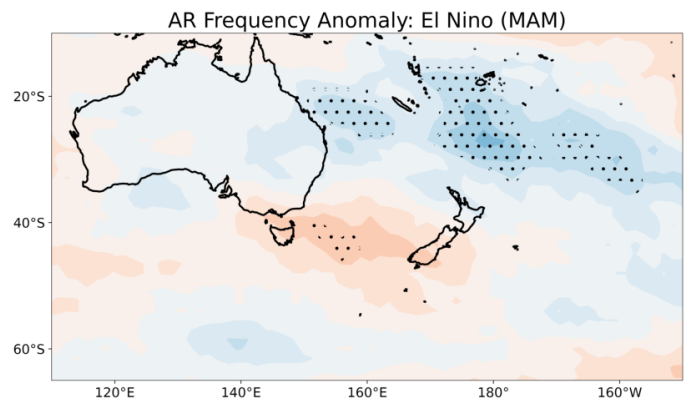

(e)

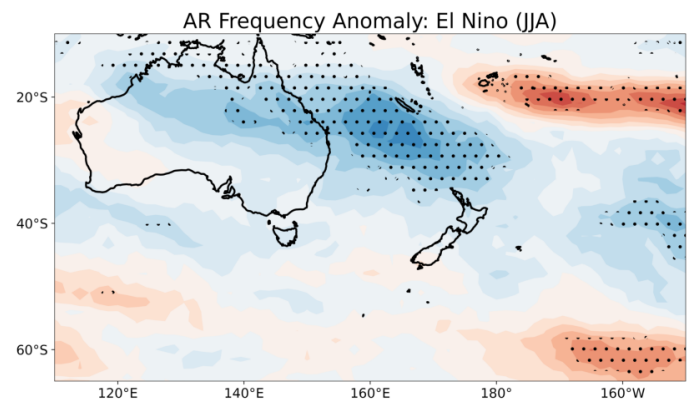

(g)

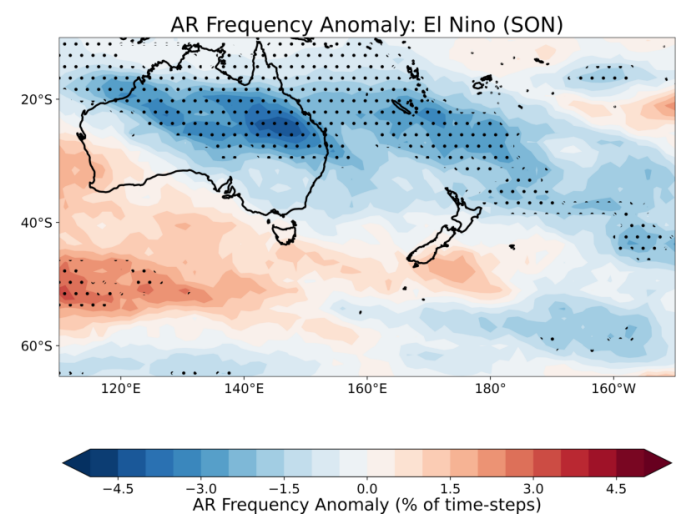

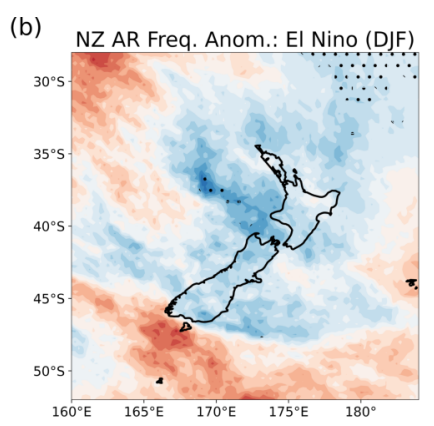

(d)

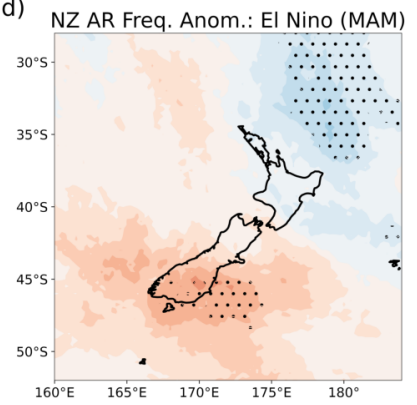

(f)

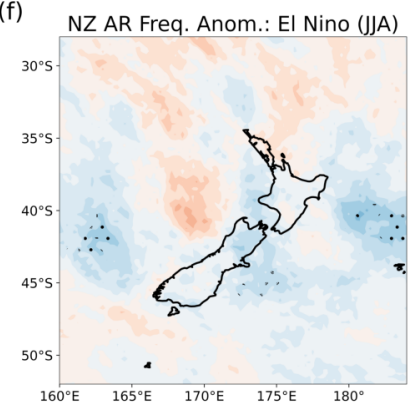

(h)

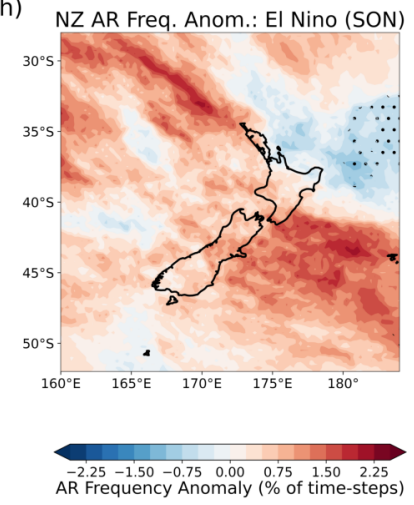

Figure 4.10: Composite AR frequency anomaly for El Niño months for each season (1979-2019). (a, c, e, g) are derived from the global AR dataset (Guan \& Waliser, 2015), while (b, d, f, h) are derived from the NZ AR dataset. Dotted areas denote anomalies significant at the $90 \%$ level. 
(a)

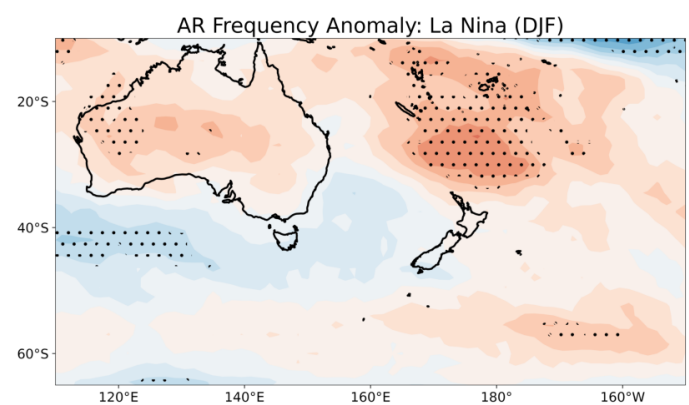

(c)

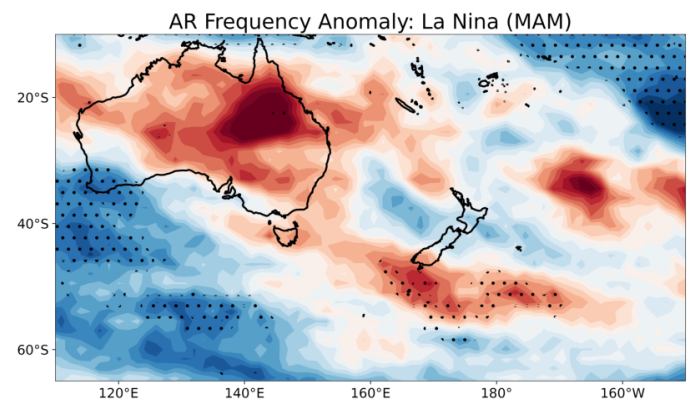

(e)

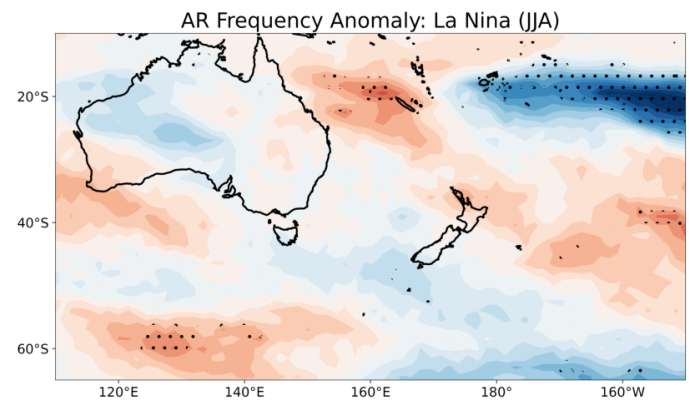

(g)

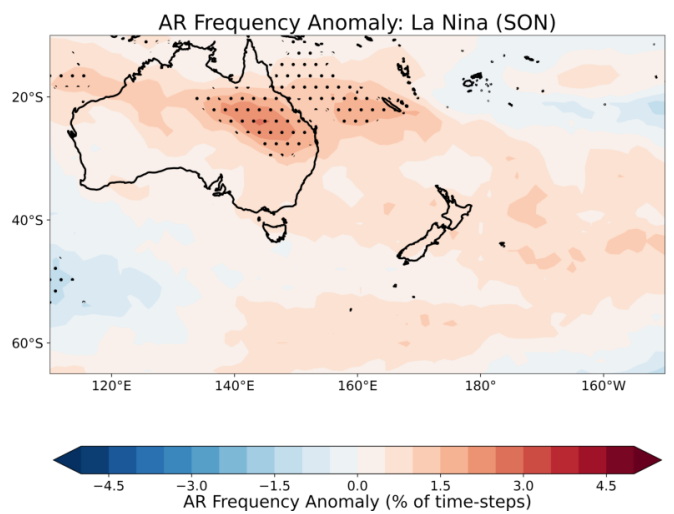

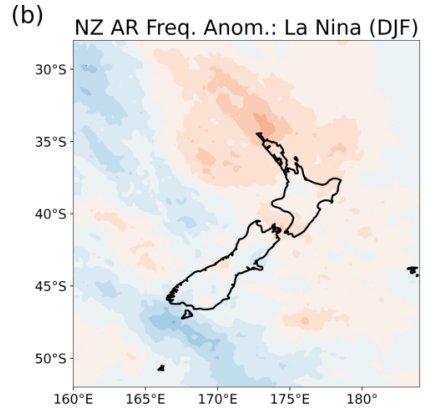

(d) NZ AR Freq. Anom.: La Nina (MAM)

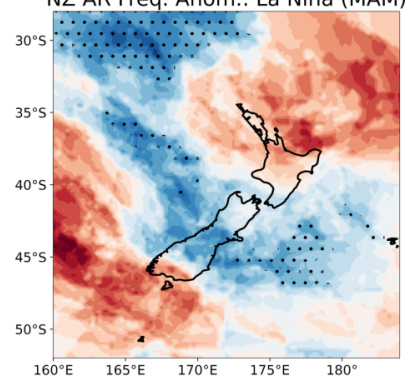

(f)

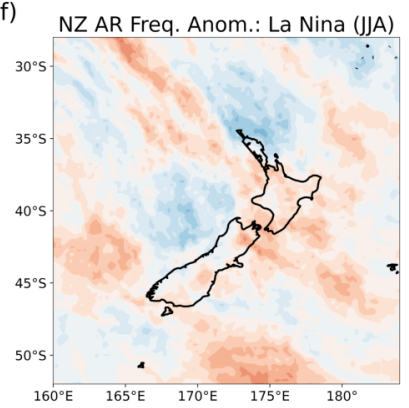

(h)

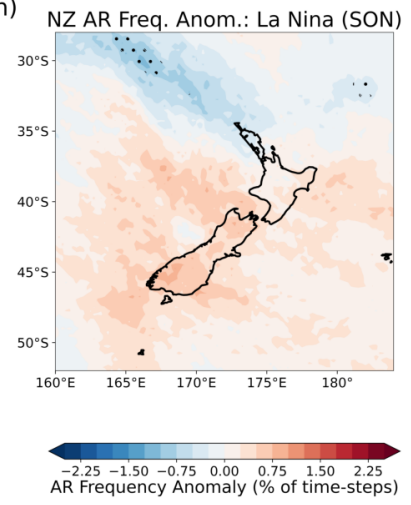

Figure 4.11: Composite AR frequency anomaly for La Niña months for each season (1979-2019). (a, c, e, g) are derived from the global AR dataset (Guan \& Waliser, 2015), while (b, d, f, h) are derived from the NZ AR dataset. Dotted areas denote anomalies significant at the $90 \%$ level. 


\subsection{IPO}

The Interdecadal Pacific Oscillation (IPO) is a natural climate oscillation over the Pacific Ocean that represents features similar to ENSO but operating on decadal time scales. The IPO is the leading principal component of monthly sea surface temperatures in the North Pacific (Mantua et al., 1997; Power et al., 1999). Each phase of the IPO tends to persist for 20-30 years before switching; a negative phase was observed between 1947-1977, replaced by a positive phase between 1978-1999, and a current return to a negative IPO phase (Mantua et al., 1997; Power et al., 1999). Although operating on different time scales, the IPO is associated with similar tropical SST distribution and impacts as ENSO. Phase changes of the IPO are strongly correlated with the El Niño-Southern Oscillation, with negative IPO phases corresponding to La Niña-like SST patterns, and positive IPO phases corresponding to El Niño-like SST patterns (Power et al., 1999). Similar to El Niño, positive IPO phases are associated with increased IVT magnitude over New Zealand (especially the North Island) forced by a lower pressure anomaly to the southeast of New Zealand. Figure 4.12 and Figure 4.13 show the seasonal IVT and MSLP anomalies associated with positive and negative IPO phases respectively.

Knowledge of how IPO modulates ARs may improve understanding of decadal patterns of freshwater resources and flood risk in New Zealand. Salinger et al. (2001) showed that positive IPO phases are associated with stronger southwesterly flow over the South Island, with the strongest influence in austral fall (MAM). Positive IPO phase increased summer precipitation for the south and west of the South Island, and reduced autumn precipitation for the northern North Island (Salinger et al., 2001).

For our analysis, we use the Tripole Index for the Interdecadal Pacific Oscillation (TPI/IPO). The index is based on the difference between SST anomalies averaged over the central equatorial pacific, and the average SST anomalies in the Northwest and Southwest Pacific (Henley et al., 2015). This index was obtained from NOAA, and can be accessed at: http://www.esrl.noaa.gov/psd/data/ timeseries/IPOTPI/.

\section{IPO AR Anomalies}

Our period of study (1979-2019) corresponds with a positive IPO phase between 1979 and 1999, and a negative IPO phase since 2000. AR frequency increased over the southwestern South Island with the positive IPO phase, especially for autumn months (MAM) ${ }^{6}$. A significant reduction in AR frequency north of New Zealand

\footnotetext{
${ }^{6}$ See Appendix B
} 
(a)

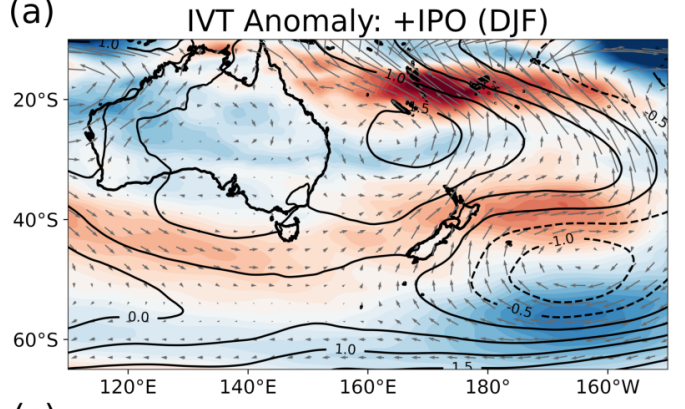

(c)

IVT Anomaly: +IPO (JJA)

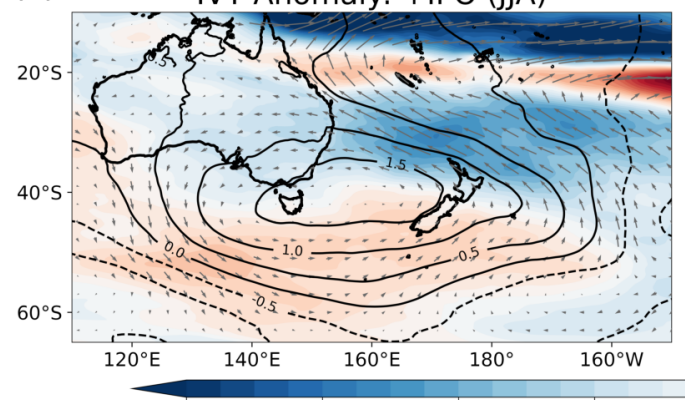

(b)

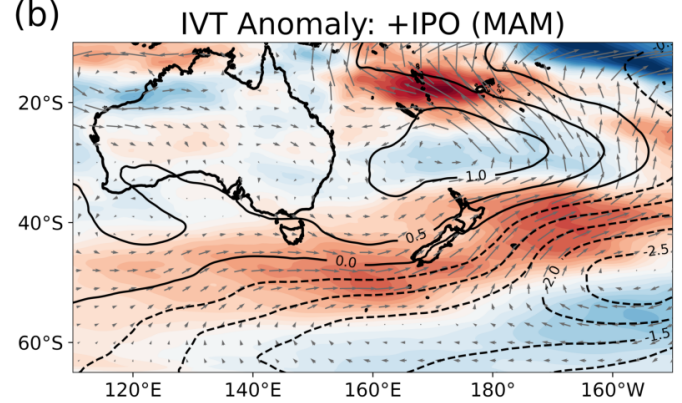

(d)

IVT Anomaly: +IPO (SON)

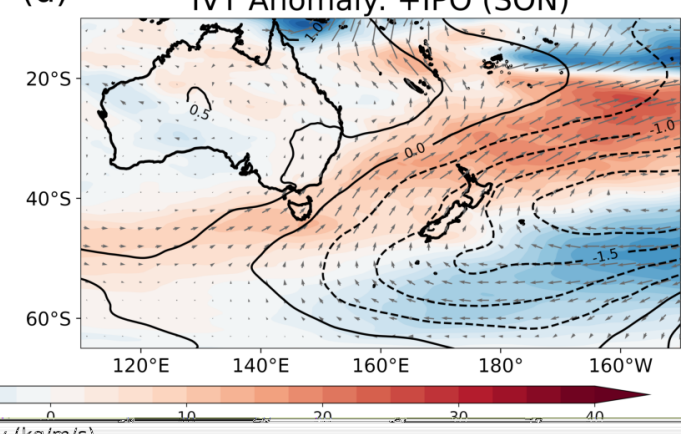

Figure 4.12: Composite IVT and MSLP anomalies for positive IPO months for each season (1979-2019). Seasonal IVT anomalies (coloured contours and vectors), and MSLP anomalies (black contours), derived from ERA5 reanalysis. 
(a)

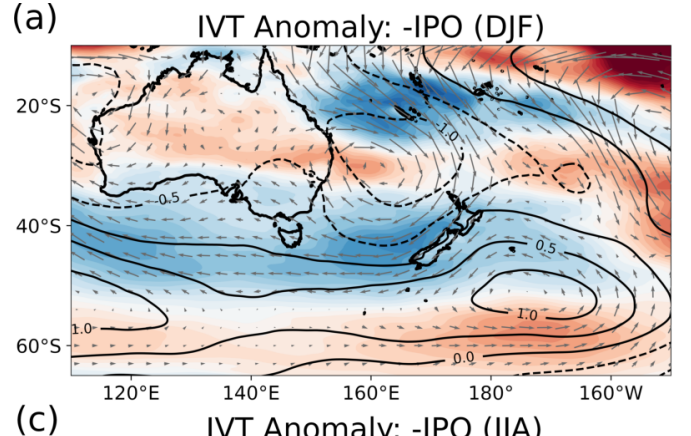

(c)

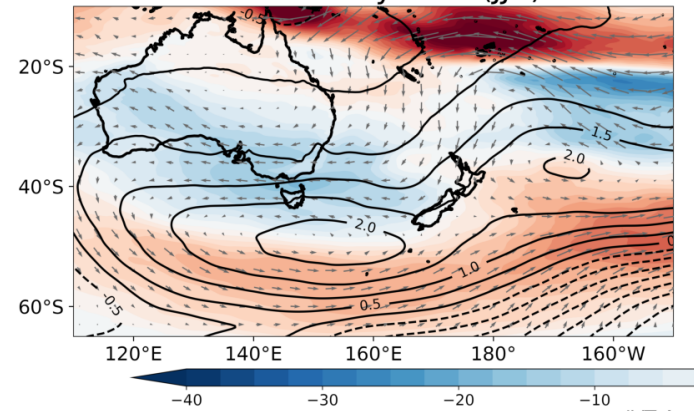

(b) IVT Anomaly: -IPO (MAM)

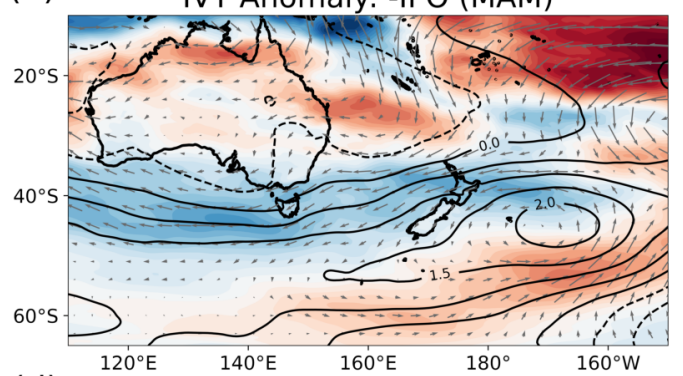

(d)

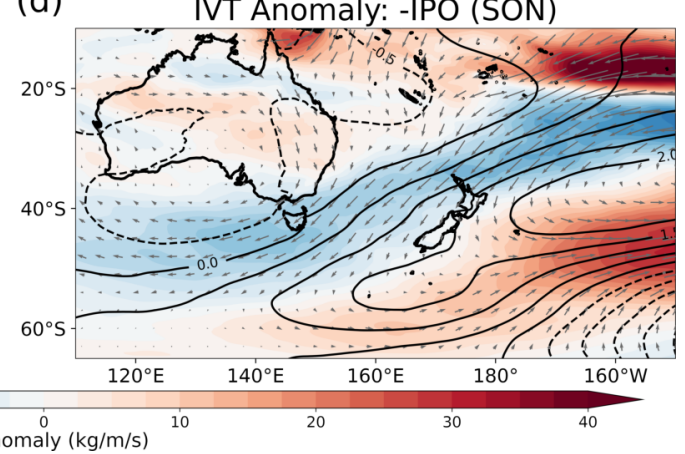

Figure 4.13: Composite IVT and MSLP anomalies for negative IPO months for each season (1979-2019). Seasonal IVT anomalies (coloured contours and vectors), and MSLP anomalies (black contours), derived from ERA5 reanalysis.

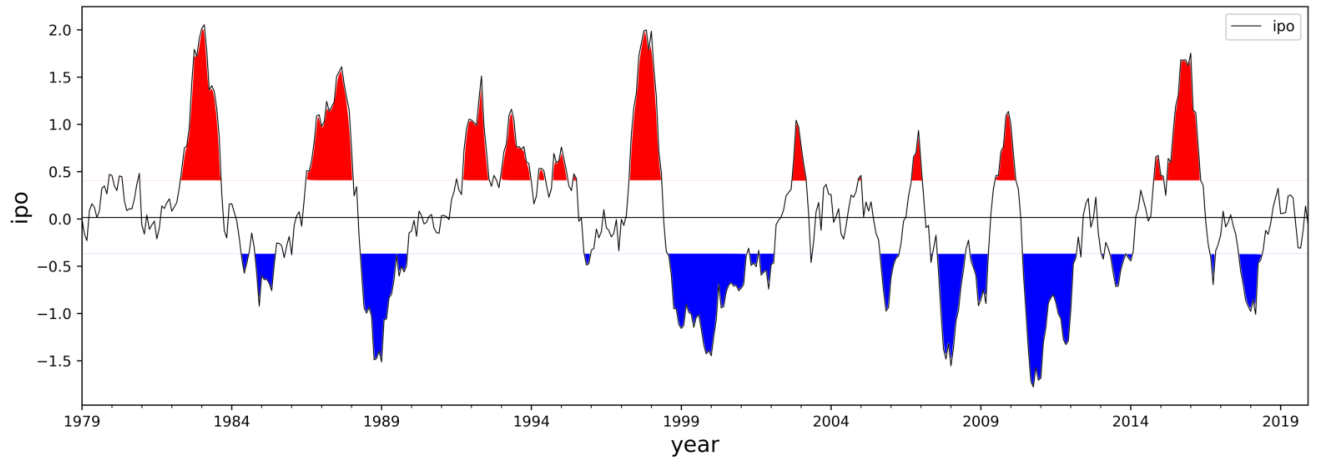

Figure 4.14: Monthly IPO index from 1979-2019. Values +/- 0.5 standard deviation are coloured. 
for the positive phase of the IPO was observed for all months. As with El Niño, there is a statistically significant decrease in the mean IVT of ARs, and the IVT magnitude at landfall. ARs were associated with more H, HNW and HE Kidson types, and fewer NE Kidson types.

The negative IPO phase is associated with significant increases in AR frequency over the North Island, especially during summer and autumn. As with La Niña, there is a statistically significant increase in the mean IVT of ARs, and the IVT magnitude at landfall. Interestingly, although more ARs make landfall over the eastern and southwestern North Island, the number of ARs contributing to 'Extreme' daily rainfall totals was reduced. ARs were associated with more HE Kidson types, and fewer T, TSW, HNW, HW and R Kidson types. Overall, the spatial distribution of the AR frequency anomalies associated with the IPO and ENSO are similar (though act over different time-scales). The analysis would likely benefit from extending the climatology to include years pre-1978 when a major shift from negative to positive IPO phase took place. 
(a)

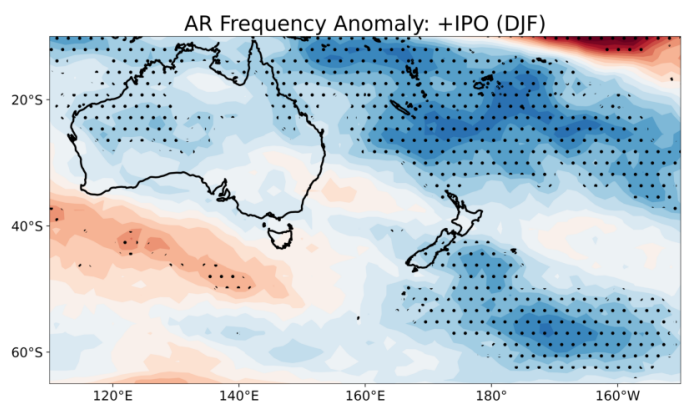

(c)

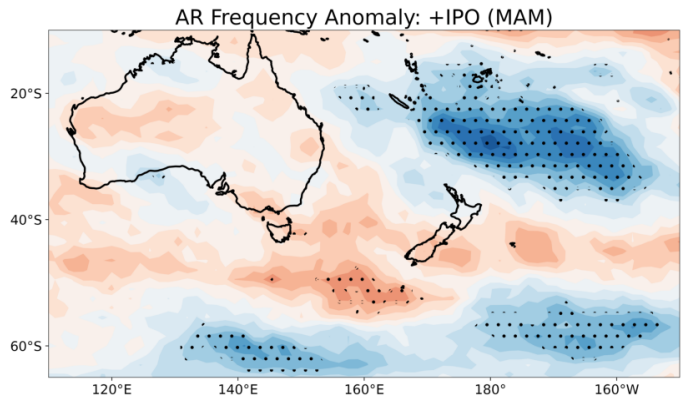

(e)

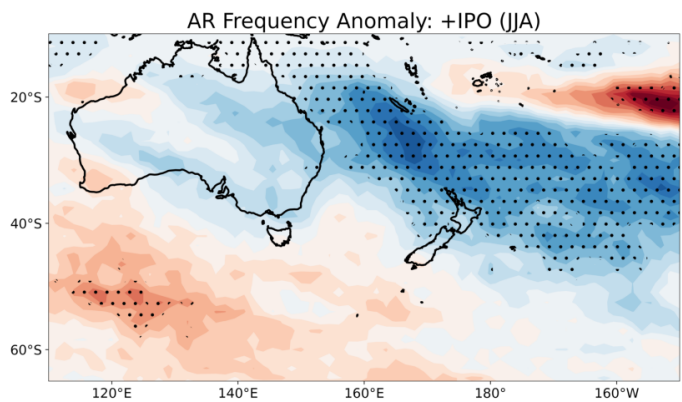

(g)

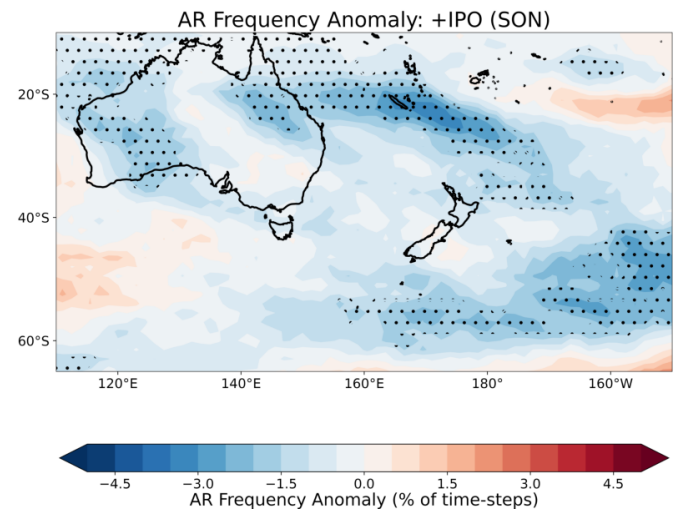

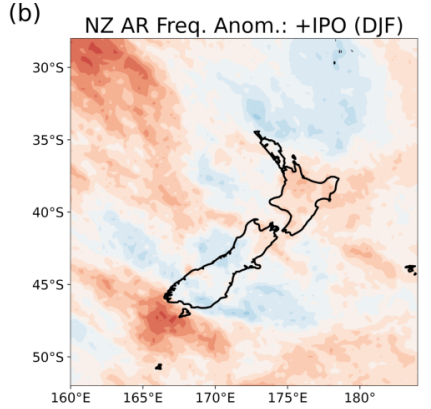

(d)

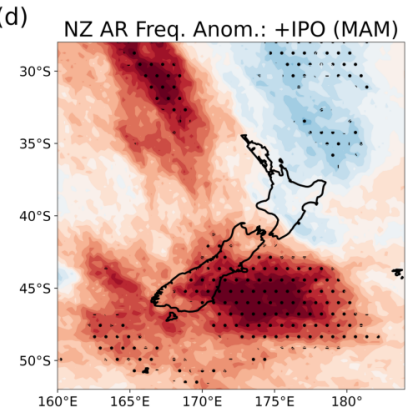

(f)

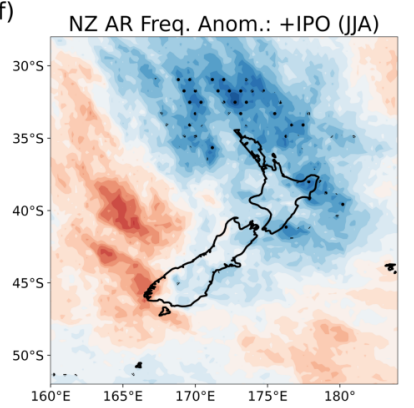

(h)

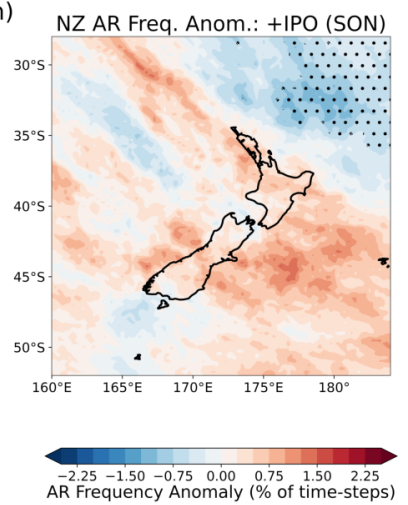

Figure 4.15: Composite AR frequency anomaly for positive IPO months for each season (1979-2019). (a, c, e, g) are derived from the global AR dataset (Guan \& Waliser, 2015), while (b, d, f, h) are derived from the NZ AR dataset. Dotted areas denote anomalies significant at the $90 \%$ level. 
(a)

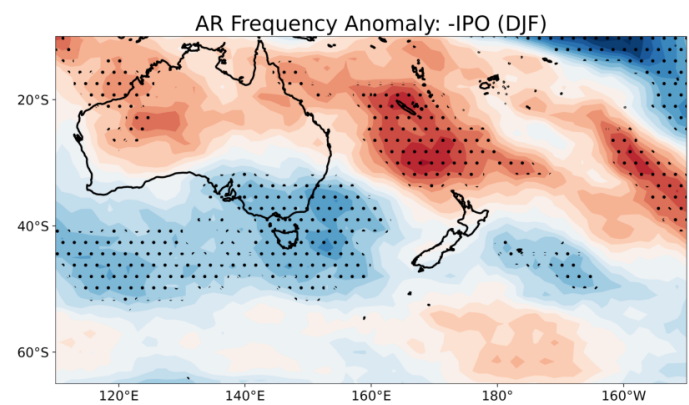

(c)

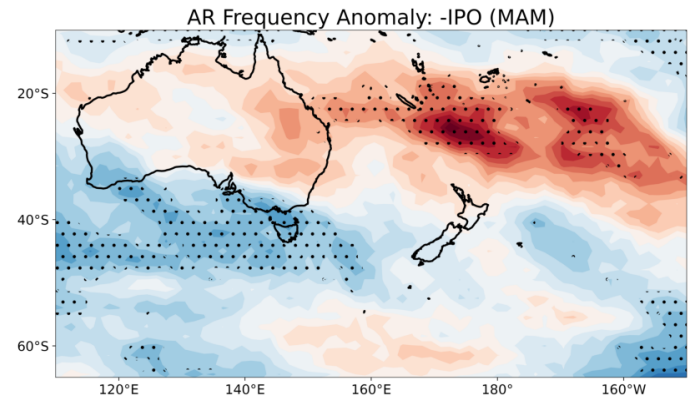

(e)

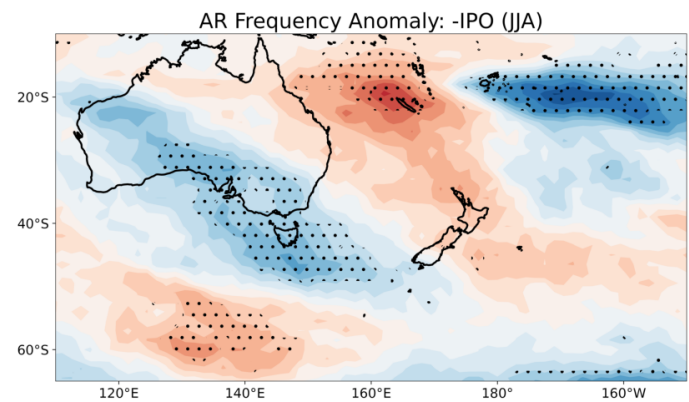

(g)

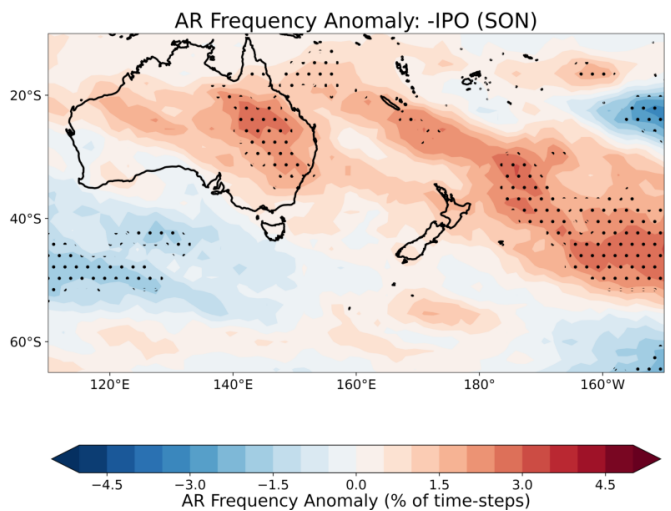

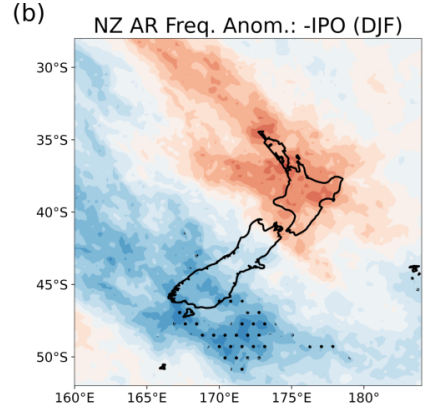

(d)

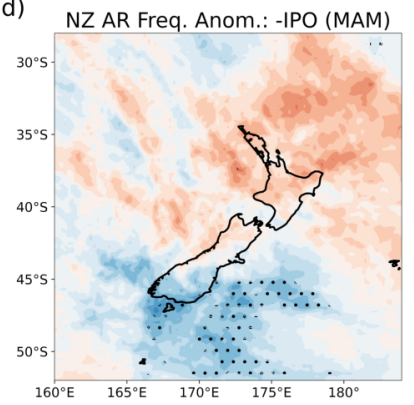

(f)

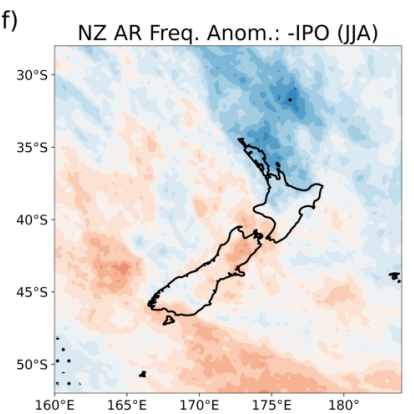

(h)

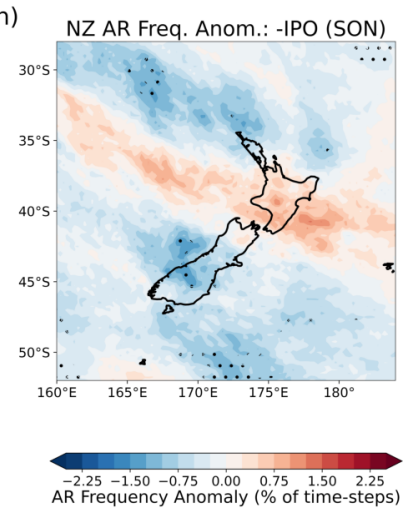

Figure 4.16: Composite AR frequency anomaly for negative IPO months for each season (1979-2019). (a, c, e, g) are derived from the global AR dataset (Guan \& Waliser, 2015), while (b, d, f, h) are derived from the NZ AR dataset. Dotted areas denote anomalies significant at the $90 \%$ level. 


\subsection{IOD}

The Indian Ocean Dipole (IOD) describes an ocean-atmosphere coupled climate oscillation related to the SST difference between the western Indian Ocean (in the Arabian Sea) and eastern Indian Ocean (south of Indonesia). The SST difference and development of IOD events tends to peak between June and October (Ashok et al., 2007). Positive IOD phases are associated with increased precipitation in the western Indian Ocean and easterly wind anomalies over the Indian Ocean, with the opposite effect during negative phases. During positive IOD events, the prevailing westerlies and storm track activity weaken over portions of New Zealand (Ashok et al., 2007). Figure 4.17 and Figure 4.18 show the seasonal IVT and MSLP anomalies associated with positive and negative IOD phases respectively.

(a)

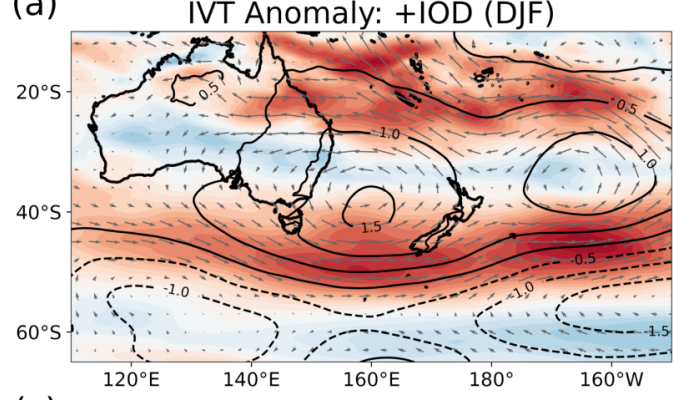

(c)

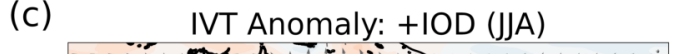

(b) IVT Anomaly: +IOD (MAM)

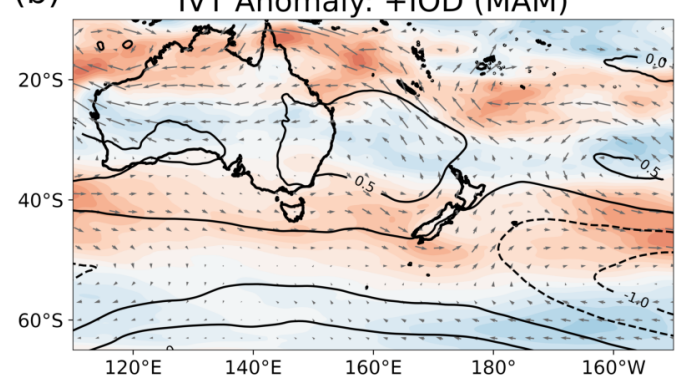

(d)

IVT Anomaly: + IOD (SON)

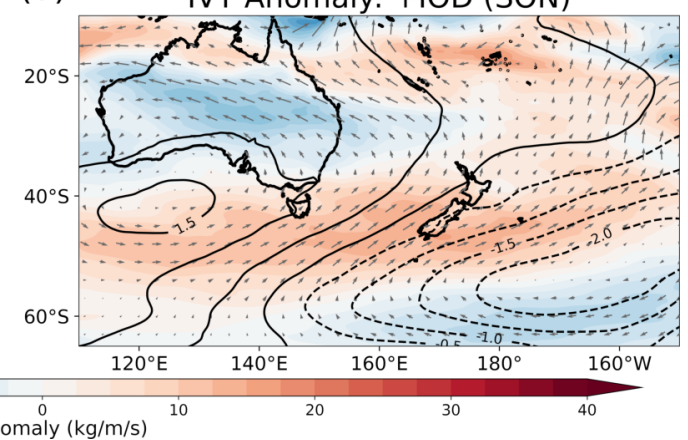

Figure 4.17: Composite IVT and MSLP anomalies for positive IOD months for each season (1979-2019). Seasonal IVT anomalies (coloured contours and vectors), and MSLP anomalies (black contours), derived from ERA5 reanalysis.

Mullan (1998) found significant teleconnections between tropical Indian Ocean SSTs and New Zealand climate, most prominent during autumn and winter. In particular, warmer Indian Ocean SSTs during autumn were associated with a stronger winter subtropical ridge north of the North Island, stronger wester- 
(a)

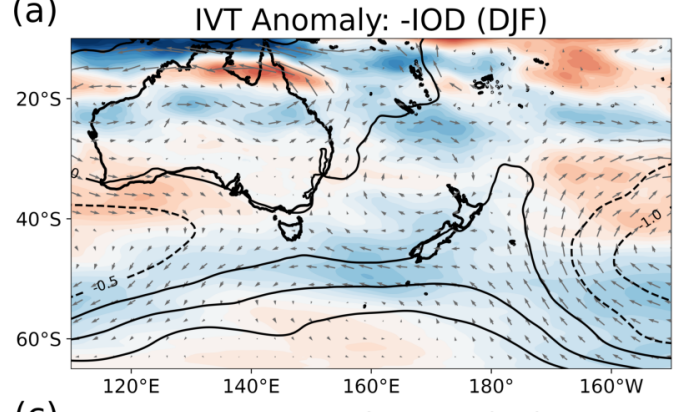

(c) IVT Anomaly: -IOD (JJA)

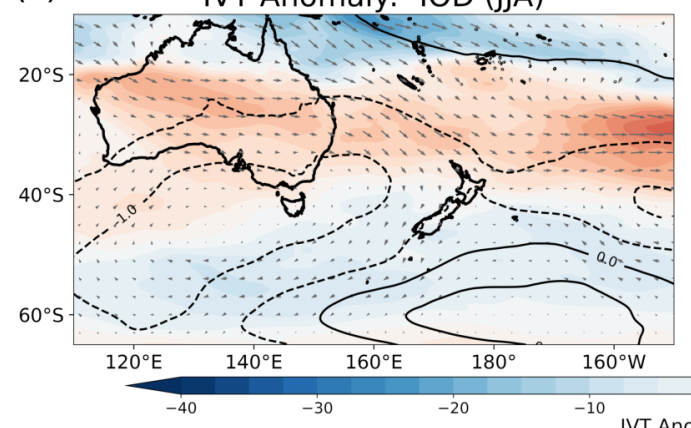

(b)

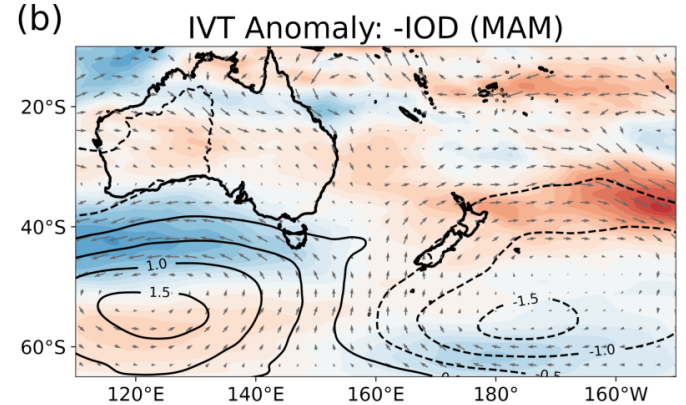

(d)

IVT Anomaly: -IOD (SON)

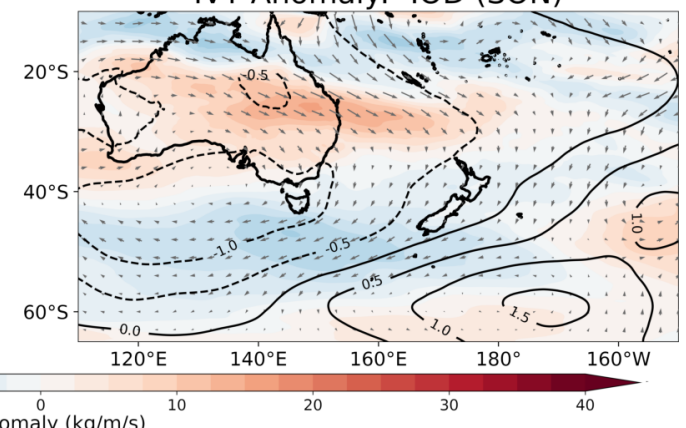

Figure 4.18: Composite IVT and MSLP anomalies for negative IOD months for each season (1979-2019). Seasonal IVT anomalies (coloured contours and vectors), and MSLP anomalies (black contours), derived from ERA5 reanalysis. 
lies, higher temperatures for the South Island, and wetter (drier) conditions for western (northeast) regions. Ashok et al. (2007) found positive IOD phases were associated with a reduction in rainfall for northern New Zealand for winter and spring. Negative IOD phases were associated with enhanced westerlies and storm track activity over northern New Zealand, and weakened storm track activity over southern New Zealand (Ashok et al., 2007). Over the northern South Island and northern North Island, the IOD had a greater influence on precipitation compared with ENSO (Ashok et al., 2007). Thus, we expect the IOD to influence the frequency and characteristics of landfalling ARs.

For our analysis, the Indian Ocean Dipole (IOD) is represented by the Dipole Mode Index (DMI) derived from the HadISST1.1 SST dataset, sourced from The NOAA Physical Sciences Laboratory (PSL). The index is based on the anomalous SST difference between the western equatorial Indian Ocean (between $10^{\circ} \mathrm{N}-10^{\circ} \mathrm{S}$ and $50^{\circ} \mathrm{E}-70^{\circ} \mathrm{E}$ ) and southeastern equatorial Indian Ocean (between $0^{\circ} \mathrm{N}-10^{\circ} \mathrm{S}$ and $\left.90^{\circ} \mathrm{E}-110^{\circ} \mathrm{E}\right)$.

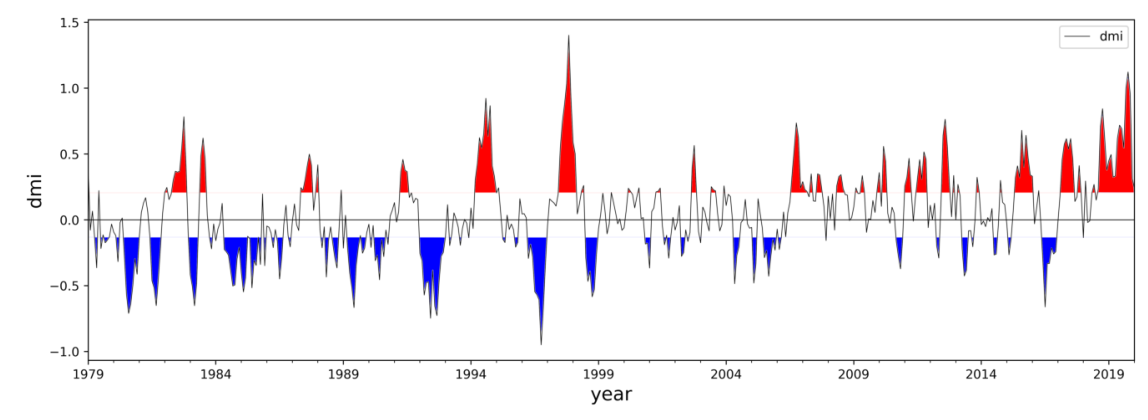

Figure 4.19: Monthly DMI IOD index from 1979-2019. Values +/- 0.5 standard deviation are coloured.

\section{IOD AR Anomalies}

Previously identified circulation changes around New Zealand include a weaker storm track north of New Zealand and lower pressure to the southeast of New Zealand during positive IOD phases. In particular, Ashok et al. (2007) found positive IOD phases were associated with a reduction in rainfall for northern New Zealand for winter and spring. The AR frequency anomalies in Figure 4.20 also reflects this pattern, with positive IOD phases associated with statistically significant decreases in AR frequency to the northwest of New Zealand for all seasons. This is also reflected in the Kidson types associated with ARs, with fewer T, TNW, TSW, and NE types. We also find that positive phases of the IOD are associated with increased IVT and AR frequency in the storm track to the south of New 
Zealand. There are significant reductions in the number of ARs contributing to 'Heavy' rainfalls in the North Island and northern South Island for all seasons. Also, significant reductions in the number of ARs contributing to 'Extreme' rainfalls in the North Island and northern South Island during summer and autumn. There is a statistically significant decrease in the mean IVT of ARs during the positive phase.

During autumn, winter and spring, the negative phase of the IOD is associated with increased AR frequency extending across Australia and to the northwest of New Zealand. Over New Zealand, no statistically significant AR count anomalies were found. Though in general, there is a reduction in the number of ARs making landfall in summer, and a slight increase in winter (not statistically significant). During the negative IOD phase, there is a statistically significant increase in the mean IVT of ARs, but a decrease in the number of ARs contributing to 'Extreme' rainfalls in the North Island and northern South Island. 
(a)

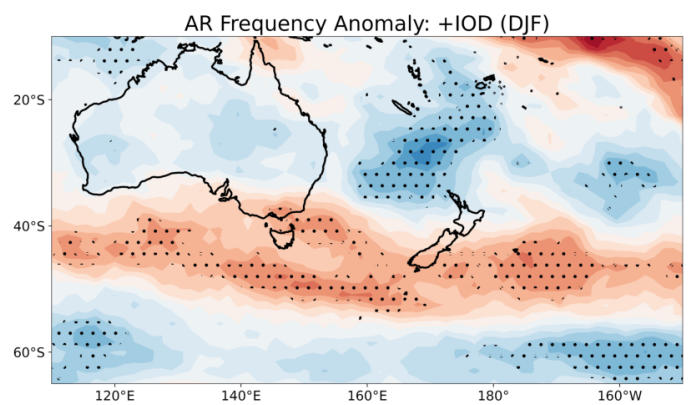

(c)

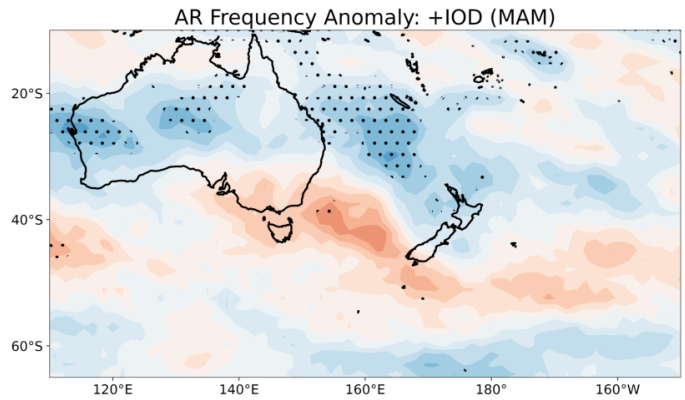

(e)

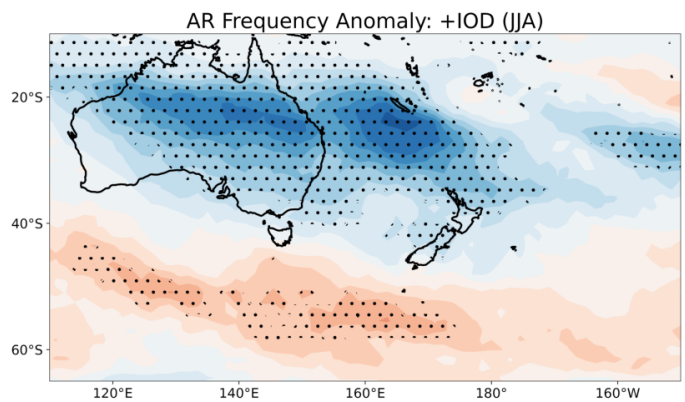

(g)

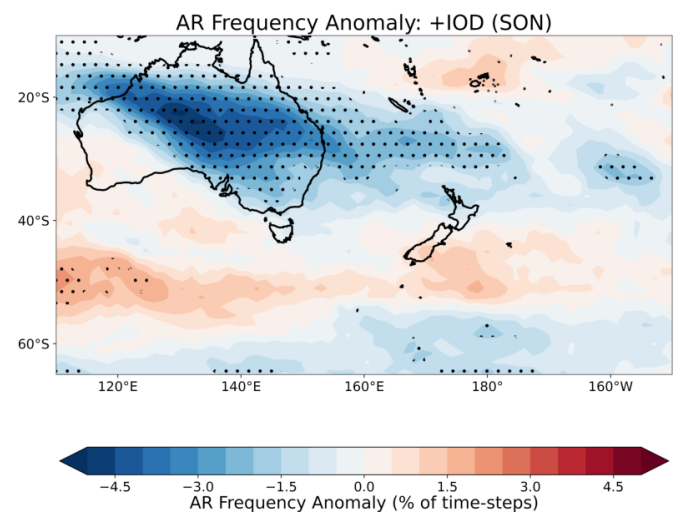

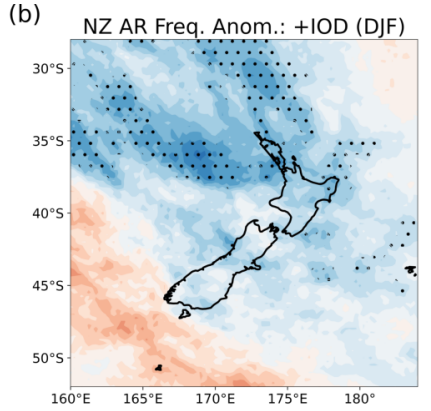

(d)
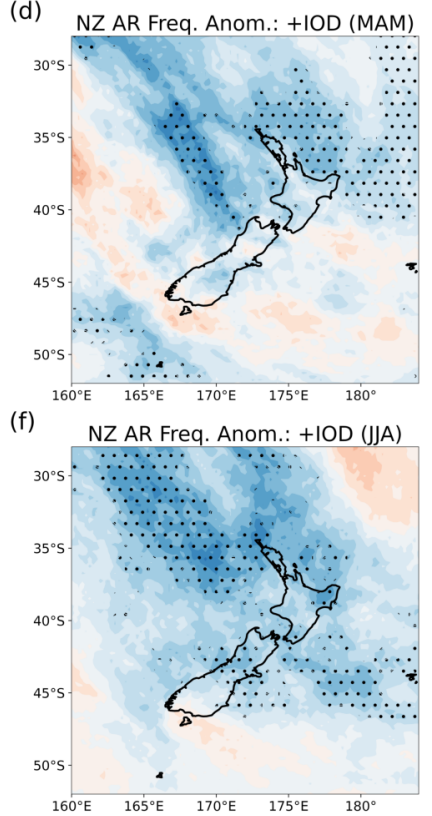

(h)

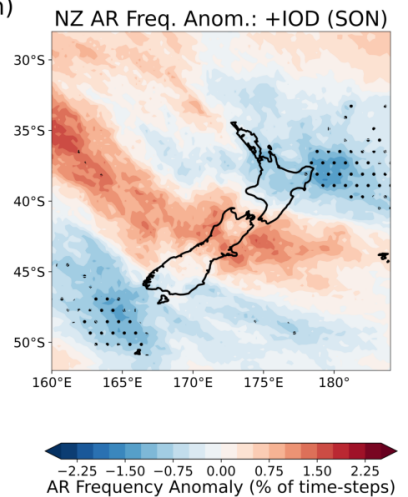

Figure 4.20: Composite AR frequency anomaly for positive IOD months for each season (1979-2019). (a, c, e, g) are derived from the global AR dataset (Guan \& Waliser, 2015), while (b, d, f, h) are derived from the NZ AR dataset. Dotted areas denote anomalies significant at the $90 \%$ level. 
(a)

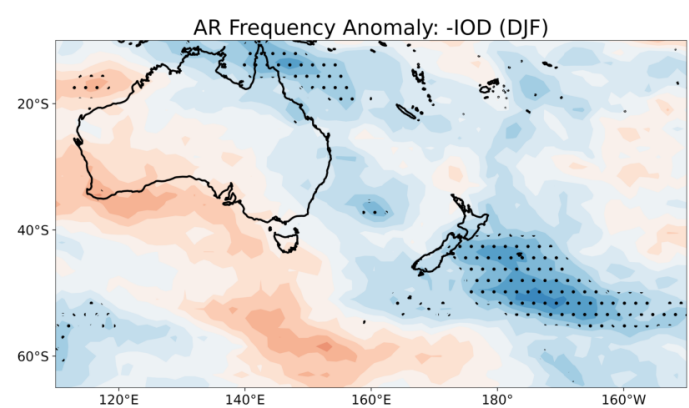

(c)

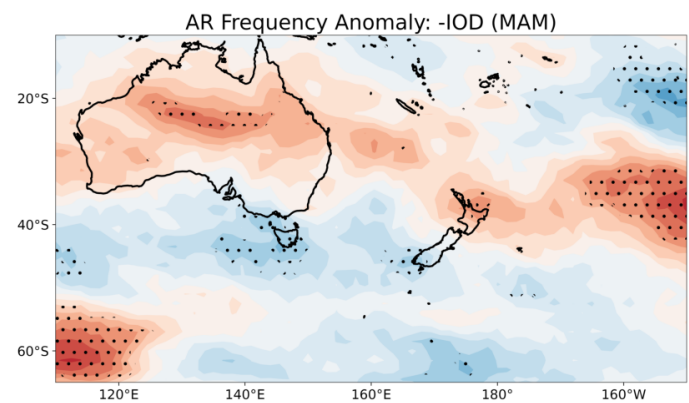

(e)

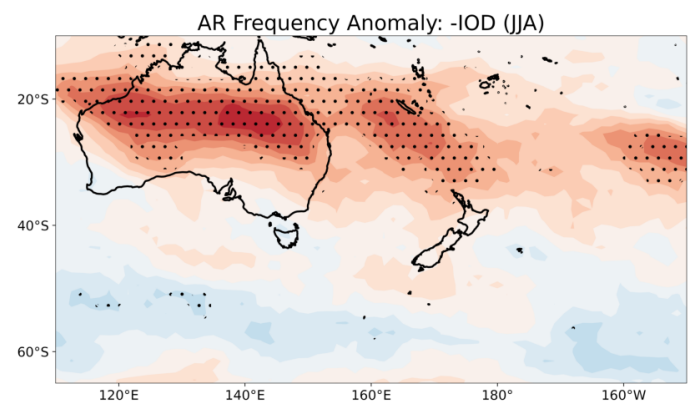

(g)

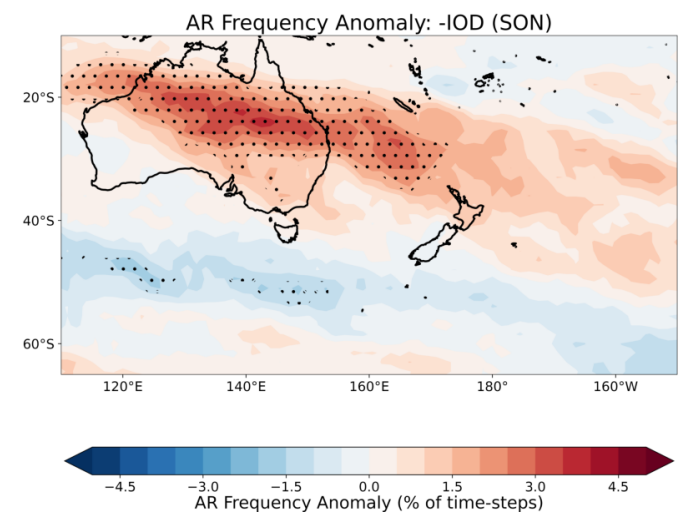

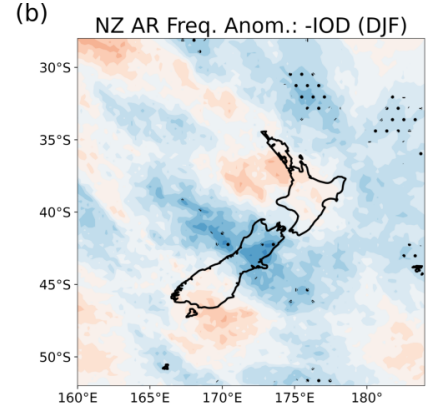

(d)

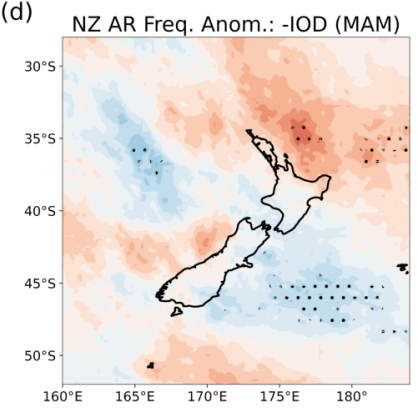

(f)

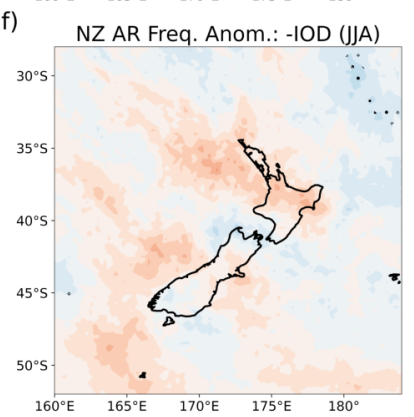

(h)

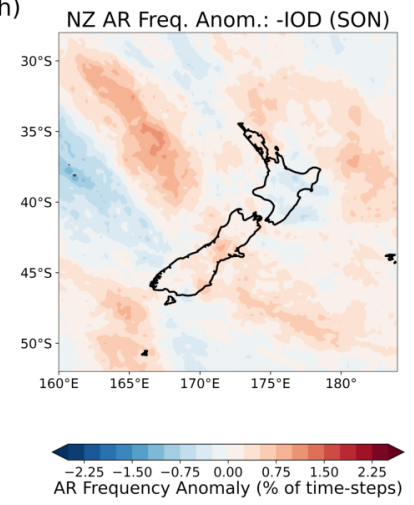

Figure 4.21: Composite AR frequency anomaly for negative IOD months for each season (1979-2019). (a, c, e, g) are derived from the global AR dataset (Guan \& Waliser, 2015), while (b, d, f, h) are derived from the NZ AR dataset. Dotted areas denote anomalies significant at the $90 \%$ level. 


\subsection{MJO}

The Madden-Julian Oscillation (MJO) is an intraseasonal (30 to 60 days) mode of tropical variability characterized by a tropical convective zone moving westto-east along the equator (Madden and Julian, 1971). Notably, the MJO provides an important source of predictability for subseasonal forecasting (Vitart, 2014). The phase and amplitude of the MJO is represented by the Real-time Multivariate MJO (RMM) index (Wheeler and Hendon, 2004) obtained from the Bureau of Meteorology. The index is based on empirical orthogonal function (EOF) analysis involving fields of $850 \mathrm{hPa}$ zonal wind, $200 \mathrm{hPa}$ zonal wind, and outgoing longwave radiation near the equator. The MJO phase represented by the RMM index coincides with the location of enhanced cloud and rainfall near the equator. During phase 1 convective activity begins over the Indian Ocean which moves eastward during phase 2 and 3. During phase 4 and 5, anomalous convection is located north of Australia, which then moves eastward across the Pacific during phase 6, 7 and 8 (Wheeler and Hendon, 2004). The MJO influences rainfall, convection, SSTs and tropical cyclone initiation in the tropics, and can impact the mid-latitudes through circulation responses to tropical diabatic heating anomalies (and generation of Rossby wave trains) (Zhang, 2005; Matthews et al., 2010). The MJO interacts with other climate phenomena across multiple time-scales, in particular enhanced MJO activity in the western Pacific has been observed to precede peak El Niño events (Hendon et al., 2007). In the Southern Hemisphere, previous investigations in South Africa (Pohl et al., 2007) and South America (Shimizu and Ambrizzi, 2016) found precipitation and convection in these regions are strongly modulated by the MJO cycle. Fauchereau et al. (2016) investigated the MJO signal over New Zealand using Kidson weather types, demonstrating strong impacts on rainfall driven by regional circulation anomalies (and orographic effects). In particular, strong increases (in some cases $40 \%-70 \%$ ) in Kidson type occurrence were associated with particular MJO phases (with a delayed response, out to lag times of 15-20 days).

A number of studies have identified relationships between the MJO and individual AR events in the western United States (Ralph et al., 2011; Guan et al., 2012). The timing of high-impacts ARs in California was found to be regulated by the MJO (with the largest impacts associated with phase 6 of the RMM index) (Guan et al., 2012). Investigations of MJO modulation of ARs in the Southern Hemisphere are limited. There is potential for the MJO to improve predictability of New Zealand weather, especially extreme events, on intraseasonal time-scales. Investigations of the MJO phase concurrent (or lagged) with AR landfalls in New Zealand, may help improve the predictability of New Zealand floods. 


\section{MJO AR Anomalies}

We investigate changes in AR frequency during the summer months, when MJO signals are at their southernmost extent (Pohl et al., 2007; Fauchereau et al., 2016). Figure 4.22 shows summer AR frequency anomalies associated with each phase of the MJO. We see a progression of positive and negative AR frequency anomalies over the MJO lifecycle. The AR frequency anomalies correlate well with the previous investigation of Fauchereau et al. (2016), which examined New Zealand precipitation anomalies associated with MJO phase. As expected, MJO phase is found to significantly influence AR frequencies north of New Zealand. These AR frequency anomalies are characterized by an eastward moving band of reduced/enhanced AR frequency to the north of New Zealand, reduced during phases 1-4 and enhanced during phases 5-8. The largest mid-latitude impacts occur downstream of the MJO convective anomalies, in particular a band of enhanced AR frequency extends across the Pacific to the Antarctic Peninsula (Guan and Waliser, 2015). Over the North Island, significant increases in AR frequency are observed for phases 3 and 5, and decreases in AR frequency for phases 2, 4 and 6. Over the South Island, significant increases in AR frequency are observed for phases 2,6 and 8, and decreases in AR frequency for phase 4. The results support the notion that MJO phase directly modulates New Zealand climate on intraseasonal time-scales.

MJO forcing takes time to propagate, and impacts may be lagged over several days to weeks (Branstator, 2014). To further examine how the MJO influences New Zealand landfalling ARs, we investigate any lagged relationships between the MJO phase and AR frequency. Again we look at summer months, when MJO signals are at their southernmost extent (Pohl et al., 2007; Fauchereau et al., 2016). Fauchereau et al. (2016) investigated lagged relationships between the MJO signal and various Kidson type circulation patterns. In the New Zealand sector, geopotential anomalies propagate from west to east, and phase speeds are consistent with the propagation of the MJO convective anomalies (Fauchereau et al., 2016). A number of Kidson types showed significant responses up to 30 days after a particular MJO phase. Quoting Fauchereau et al. (2016); "at lag 0 for phase 3 of the MJO, TSW is less frequent than climatology, while $\mathrm{H}$ and $\mathrm{W}$ are both more frequent. At about 17-23-day lags, a strong opposite response is observed, with large increase in the occurrences of TSW and decrease in the occurrences of $\mathrm{H}$, $\mathrm{W}$, and HNW. The following MJO phase (4), the maximum response is found at lags of about 12-18 days, while it is present to some extent about 7-12 days after phase 5, around 3-5 days after phase 6, and finally a near-synchronous response is observed during phase 7". Thus, through a delayed response, the MJO influences the circulation patterns in New Zealand and alters the frequency of certain Kidson types. 

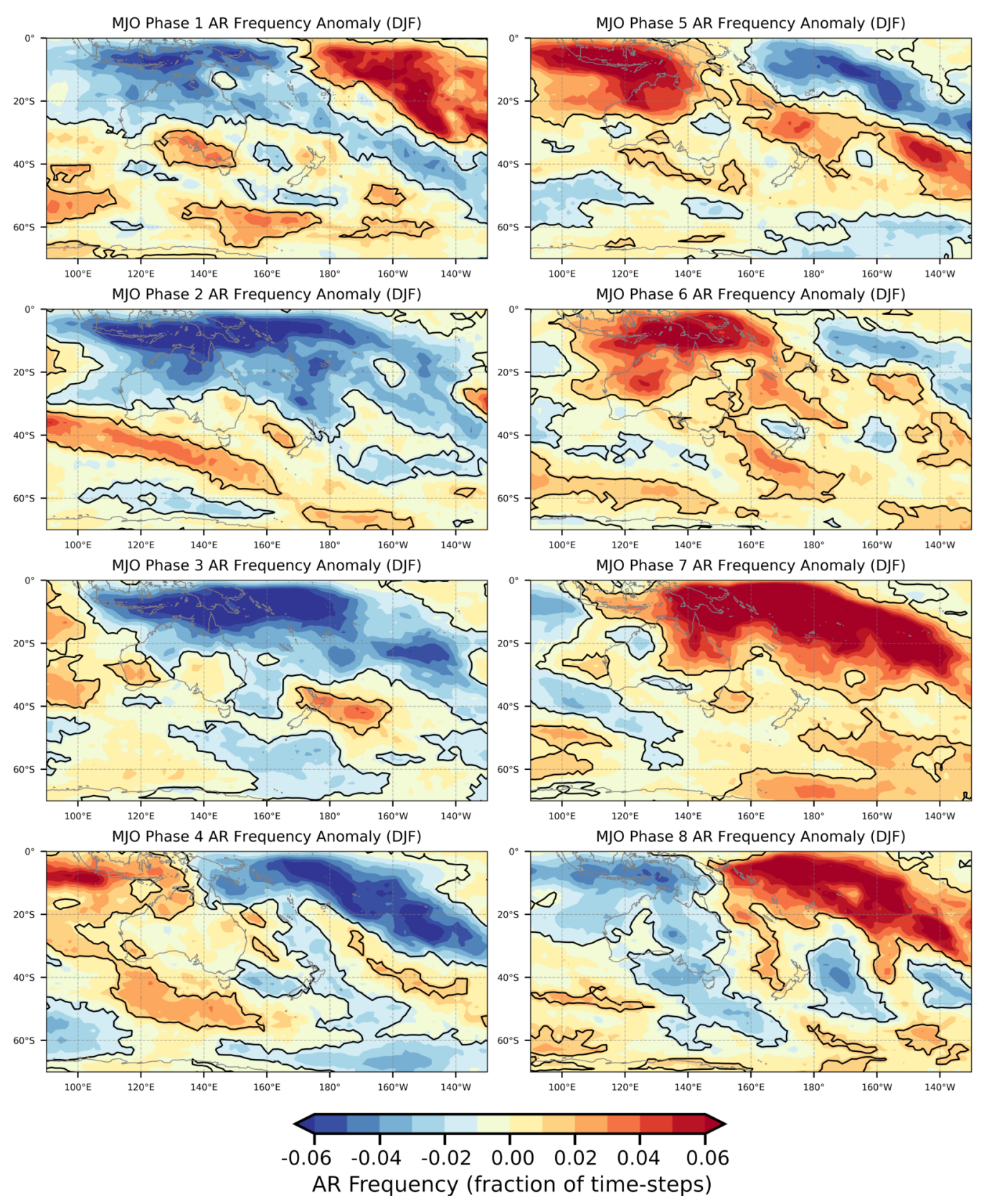

Figure 4.22: Summer (DJF) composite AR frequency anomalies for each phase of the MJO (1979-2019), derived from the global AR catalogue (Guan \& Waliser, 2015). Black contours show anomalies significant at the $90 \%$ level. 
As ARs are largely influenced by geopotential anomalies and circulation patterns in New Zealand, we expect to see significant lagged relationships between the MJO phase and AR frequency. To investigate regional differences in the MJO forcing we focus on the northern North Island and western South Island regions. Indeed, we find $\mathrm{MJO}$ impacts vary significantly by region, with stronger impacts for the north of New Zealand. Figures 4.23 and 4.24 show AR frequency anomalies at lag times up to 30 days. These anomalies are calculated with respect to the mean summer (DJF) AR frequency for each region. Both regions show a notable slope with lag, indicating significant MJO forcing of AR occurrence (consistent with Figure 4.22). In particular, the strongest signals are observed at lag times of 9-16 days. As expected, the MJO signal is much stronger for the northern North Island, where tropical effects are more dominant. Following MJO phase 4, the maximum response in AR frequency is found at a lag time of approximately 12 days. Indeed, Fauchereau et al. (2016) found increases in the occurrence of TNW, TSW, NE and HW Kidson types at the same lag time following phase 4 . We know these particular circulation patterns to be favourable for AR landfalls in the northern North Island (see Chapter 2).

Notably, we find significant AR frequency anomalies for the northern North Island up to 30 days after MJO phase 1 . The timing is consistent with the MJO propagation speed, with a life-cycle of 30-60 days. With signals exceeding $+60 \%$ in some cases, the findings suggest significant potential for the predictability of AR occurrence associated with the MJO, particularly for the northern North Island.

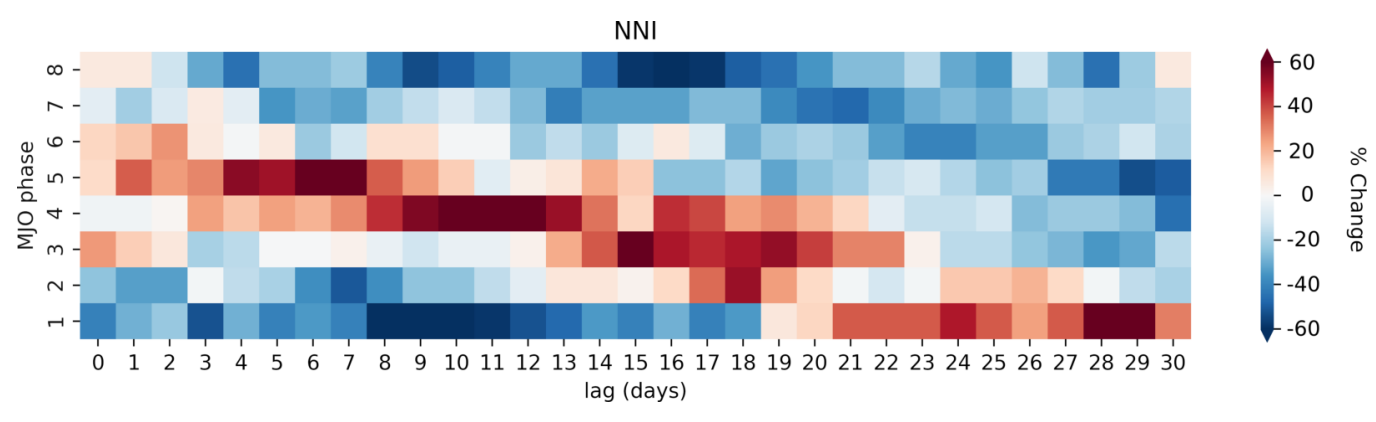

Figure 4.23: Composite AR frequency anomalies (\% change) for each MJO phase, for lag times up to 30 days. Values are calculated for ARs making landfall in the northern North Island during summer (DJF). Anomalous frequency of AR occurrence (\% change) is calculated relative to the region's mean summer AR frequency (1980-2018). 


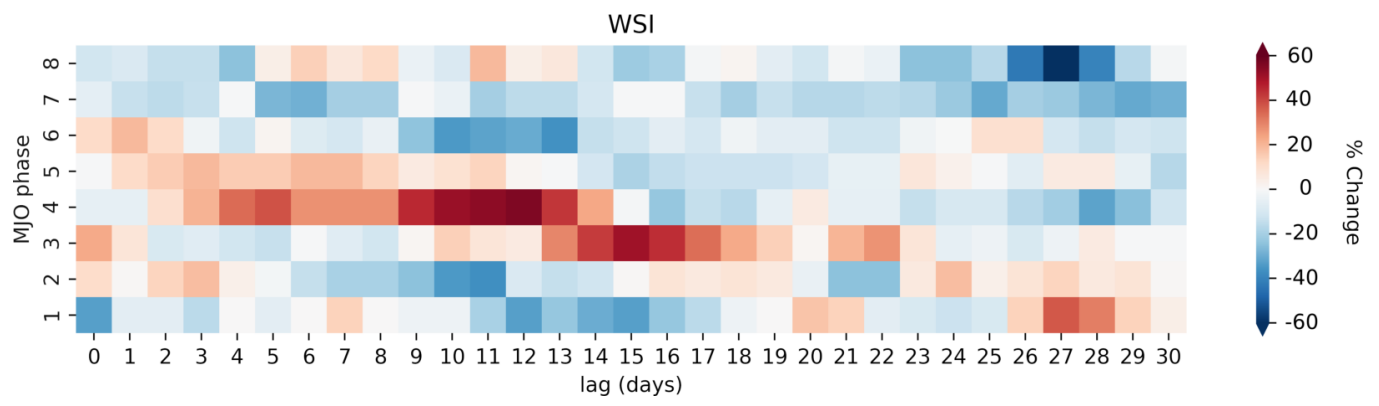

Figure 4.24: Composite AR frequency anomalies (\% change) for each MJO phase, for lag times up to 30 days. Values are calculated for ARs making landfall in the western South Island during summer (DJF). Anomalous frequency of AR occurrence (\% change) is calculated relative to the region's mean summer AR frequency (1980-2018).

\subsection{Interaction between Climate Oscillations}

Each of the climate oscillations described above are interconnected across various spatial and temporal scales, and have modulating effects on each other. Further, each climate oscillation is modulated by the annual seasonal cycle, and influenced by long-term changes in the Earth's climate. Thus, AR variability can be compounded or negated by the competing influence of each climate mode. Significant correlations between different climate oscillations have been observed. The most significant interaction is between the IPO and ENSO, due to similarities in the expression of tropical Pacific SSTs associated with each mode (though on different time scales) (Power et al., 1999). Salinger et al. (2001) found significant correlations between the phase of the IPO and the frequency of El Niño events (with the frequency of El Niño months increasing from $12 \%$ to $27 \%$ during positive IPO phases). Salinger et al. (2001) also found that ENSO-climate correlations in the New Zealand region showed considerable differences between the two phases of the IPO, with stronger teleconnections during the positive IPO phase. Yuan and Li (2008) found positive (negative) IOD events and El Niño (La Niña) events to be mostly independent during 1948-1969. However, after 1970 a stronger interaction was observed, and positive IOD events tended to occur in the same year as El Niño events. It was proposed that this changing relationship was due to an enhanced Walker circulation since 1970, increasing the linkage between SSTs in the eastern Indian Ocean and western Pacific (Yuan and Li, 2008). Tropical SST anomalies associated with ENSO also strongly influence the SAM, by modulating the mean zonal winds (Kidston et al., 2009). Enhanced MJO activity in the western Pacific has been observed to precede peak El Niño events (Hendon et al., 2007). A number of studies have investigated how the MJO modulates the SAM, and while statistical relationships exist (MJO convection in the Indian Ocean is related to decreasing SAM index values) (Matthews and Meredith, 2004; 
Flatau and Kim, 2013), MJO impacts over New Zealand were not found to be significantly modulated by the SAM (Fauchereau et al., 2016). Knowledge of the state of these climate modes, and their interactions, has potential to be applied in skilful forecasts of AR occurrence and extreme rainfall in New Zealand.

\subsection{AR Frequency Trend}

Figures 4.25 and 4.26 show the AR frequency trend in the New Zealand region since 1979. Statistically significant increases in AR frequency have been observed over southwestern New Zealand, and to the northeast of New Zealand. Of particular interest, we see a positive trend in the number of ARs per year recorded by the NZ detection algorithm (ARs detected with IVT $>95$ th percentile), over the whole country. We also investigate any trends in the mean IVT magnitude and IVT magnitude at landfall. We find statistically significant increases in the mean IVT of summer ARs for all regions, particularly for the South Island (equivalent to a mean increase of approx. $70 \mathrm{kgm}^{-1} \mathrm{~s}^{-1}, \mathrm{p}<0.05$ ). Statistically significant increases in the IVT magnitude at landfall are also observed for South Island ARs during autumn and spring (equivalent to a mean increase of $100-160 \mathrm{kgm}^{-1} \mathrm{~s}^{-1}$, $\mathrm{p}<0.15$ ). In a global study of anomalous moisture uptake associated with ARs, Algarra et al., (2020) found a statistically significant increase in evapotranspiration rates associated with New Zealand landfalling ARs between 1980-2017. Thus, anomalous uptake of moisture likely contributed to the observed AR frequency trend, and intensification of ARs. As IVT consists of dynamic (horizontal wind) and thermodynamic (moisture) components, future AR frequency will be linked to both changes in global circulation patterns and changes in mean atmospheric temperatures (Ma et al., 2020).

Observed trends in AR frequency are likely due to the recent poleward shift of the Southern Hemisphere storm track during austral summer (Solman and Orlanski, 2014). Over the last 60 years, a strengthening and poleward contraction of the circumpolar westerlies during summer has been observed, linked to the recent positive trend in the SAM (Marshall, 2003). This change is mainly attributed to depletion of ozone in the lower stratosphere over Antarctica (Thompson et al., 2011). During Antarctic winter, the cold conditions over the continent lead to the formation of polar stratospheric clouds (PSCs) that are favourable for ozone depletion when hit with ultraviolet radiation (Poole and McCormick, 1988). The Antarctic ozone hole forms in September-November, due to chemical reactions occurring on PSCs when the sun rises over the polar cap during spring (Gillett and Thompson, 2003). This springtime reduction in stratospheric ozone reduces absorption of ultraviolet radiation, induces cooling over the polar cap, and lowers pressure, resulting in a strengthening of the stratospheric polar vortex (Gillett and Thompson, 2003). This signal can propagate into the troposphere during summer 


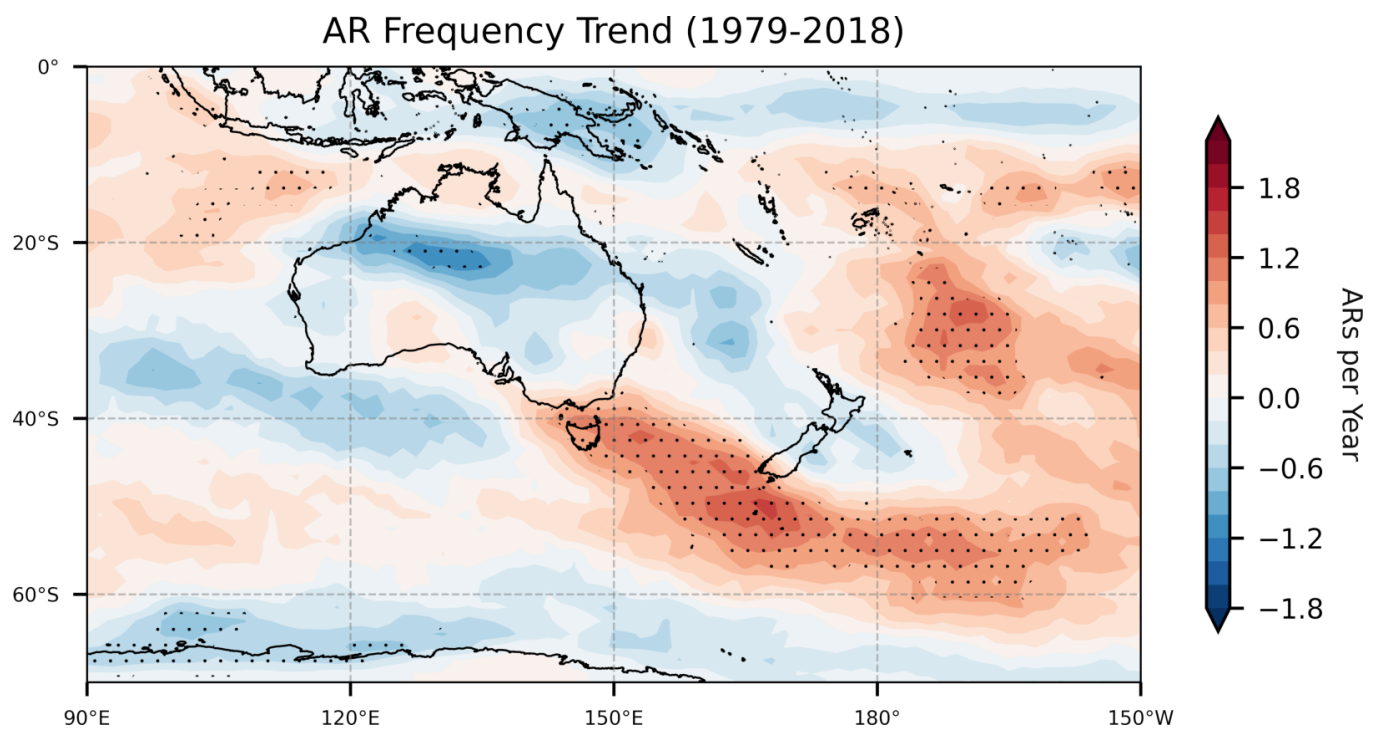

Figure 4.25: Trend in the number of ARs per year (1979-2018). Based on linear regression, values significant at $\mathrm{p}<0.05$ are indicated by black dots. Derived from the global AR dataset of Guan \& Waliser (2015).

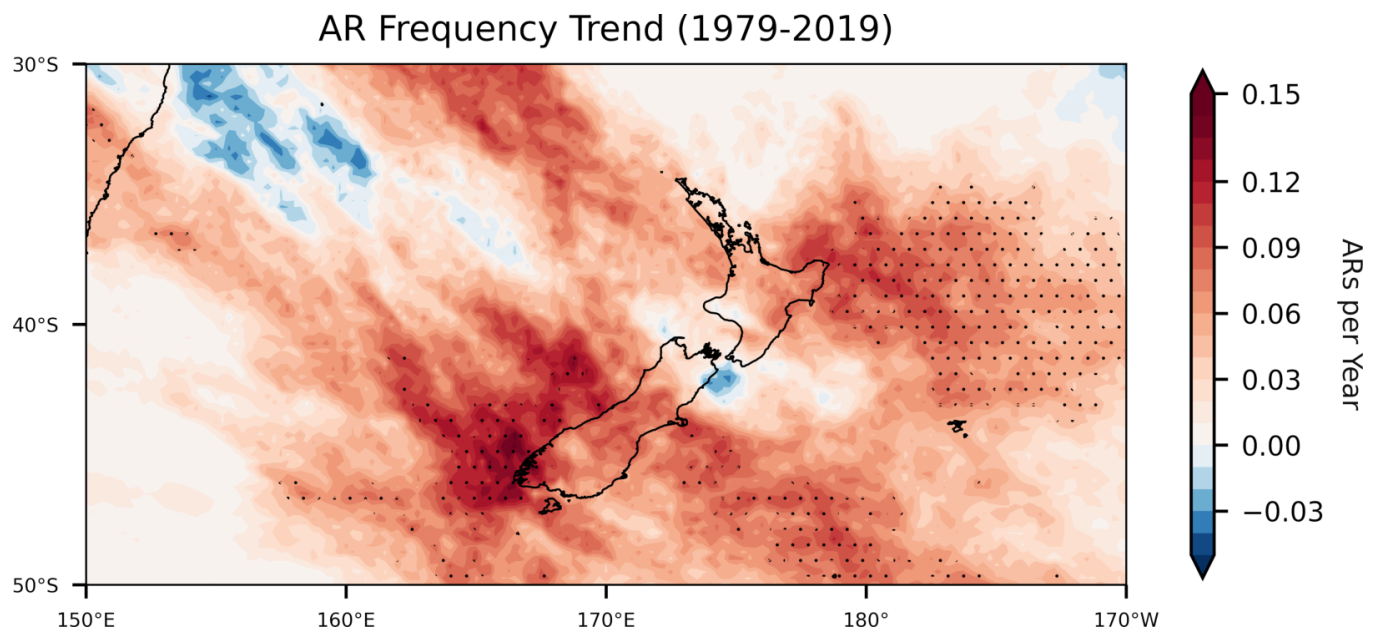

Figure 4.26: Trend in the number of ARs per year (1979-2019). Based on linear regression, values significant at $\mathrm{p}<0.05$ are indicated by black dots. Derived from the NZ AR dataset (Kennett, 2020). 
and to a lesser extent can persist into autumn, strengthening the westerly circumpolar flow (Thompson et al., 2011). A recovery of the Antarctic ozone layer in the last 30 year during September (due to phasing out of industrial chlorofluorocarbons) has weakened the summer positive SAM trend since 2000 (Solomon et al., 2016). In the future, greenhouse gas increases and recovery of the ozone hole are expected to have significant but opposing impacts on the SAM. Increases in greenhouse gas concentrations cool the stratosphere and warm the tropical troposphere. This enhances the temperature gradient between the tropics and poles, thereby increasing the circumpolar westerly winds (to maintain thermal wind balance) and contributing to a positive SAM trend (Shindell and Schmidt, 2004). At current emission rates, greenhouse gas increase will eventually dominate over the ozone signal, driving a strong tendency toward positive SAM (Thompson et al., 2011). However there is a seasonality in the competing influence of these external forcings in the future; the ozone hole's influence is only in summer, while the influence of an increase in greenhouse gas concentration is year round (Shindell and Schmidt, 2004). Therefore future projections of the SAM during austral summer are less certain (Fogt and Marshall, 2020). Yang et al. (2020) found that tropical SST variability, namely the recent period of predominantly negative IPO conditions, can also act to shift the Southern Hemisphere summertime eddydriven jet poleward. Thus, tropical variability (IPO) may have also contributed to the observed AR frequency trend.

Global climate model predictions suggest that increased mean atmospheric temperatures will increase the water vapour content available in the atmosphere (in accord with the Clausius-Clapeyron equation), increasing the mean intensity of ARs (Espinoza et al., 2018; Payne et al., 2020). The Clausius-Clapeyron relation describes how the water vapour content of saturated air changes with temperature; typically at a rate of approximately $7 \% \mathrm{~K}^{-1}$ for temperatures in the range of the troposphere (Wallace and Hobbs, 2006). Thus, increased mean atmospheric temperatures are expected to increase the mean intensity of ARs. While there is a strong relation between IVT magnitude and the distribution of ARs, an increase in mean IVT may not necessarily translate to increased AR frequency. Indeed, changes in the global atmospheric circulation under a warming climate are much less certain (Shepherd, 2014). This is partly due to the complex interactions between each climate mode, as alluded to in the last section. Further, the response of the mid-latitude storm track to (conflicting ${ }^{7}$ ) changes in the meridional temperature gradient of the lower and upper troposphere remain uncertain (Payne et al., 2020).

Espinoza et al. (2018) investigated the global-scale impacts of various climate change projection scenarios on AR frequency and intensity. Globally, future ARs will be $25 \%$ longer, $25 \%$ wider, with stronger mean IVT under the Representative

\footnotetext{
${ }^{7}$ See for example Held (1993)
} 
Concentration Pathway (RCP) 8.5 warming scenario. Climate model projections indicate a $10 \%$ reduction in the number of ARs globally in the future, with some regions experiencing considerably more ARs due to changes to the mid-latitude circulation patterns (Espinoza et al., 2018). Payne et al. (2020) provides a theoretical overview of projected changes in AR behaviour, investigating the projected changes in mean IVT under the RCP8.5 scenario. For New Zealand, thermodynamic changes are expected to increase mean IVT by up to $100 \mathrm{kgm}^{-1} \mathrm{~s}^{-1}$. Dynamic responses to warming are expected to increase IVT to the south of New Zealand, but decrease IVT significantly over most of the country (particularly for the Far North) (Payne et al., 2020). As expected, these dynamical changes show a similar spatial distribution to the positive phase of the SAM (Figure 4.2). These changes are expected to contribute to an overall increase in AR frequency in New Zealand. Associated with these changes; increased precipitation extremes, flooding, and snow/ice melt due to AR landfalls are projected (Payne et al., 2020).

It should be noted that there is significant uncertainty associated with these projections as representations of AR circulation drivers and AR processes remain limited (Payne et al., 2020). Further research on the multiscale drivers of ARs, particularly in the understudied southwestern Pacific region, is required to build confidence in future projections of AR characteristics. This is especially important as future flood frequency is recognized as a key risk for New Zealand (Reisinger et al., 2014). Current extreme rainfall projections in New Zealand under a local $2^{\circ} \mathrm{C}$ warming suggests that 100-year extreme events (i.e. events with a 1 in 100 chance of being equaled or exceeded in any given year) could occur approximately twice as often (Bell et al., 2017). Drought frequency in parts of New Zealand is also expected to increase; "time spent in drought in eastern and northern New Zealand is projected to double or triple by 2040" (Reisinger et al., 2014). In the future, the impacts of the climate oscillations described in this chapter will be superimposed on top of the climate change impacts. Future research on the projected changes to these climate drivers, and New Zealand ARs should therefore remain a priority.

\subsection{Conclusions}

For the first time in New Zealand, we investigate how large-scale climate drivers influence ARs and AR-related precipitation extremes. We examine landfalling AR frequency using two AR datasets over the 1979-2019 period, complemented by a record of precipitation observations from 189 stations, and Kidson weather types to quantify changes in AR circulation features. We find that changes in the leading modes of climate variability can alter seasonal and regional AR frequency by upwards of $30 \%$. The SAM is identified as the dominant driver of AR activity (other than the seasonal cycle), with the positive SAM phase associated with a $16 \%$ reduction in AR occurrence during summer $(30-35 \%$ for the North Island). The links between AR occurrence and the IOD, IPO, and ENSO were less 
clear, though a few statistically significant relationships were found. The MJO, the leading mode of intraseasonal tropical variability, was found to significantly influence the frequency and timing of AR landfalls (particularly for the northern North Island). Favourable MJO phases were associated with positive AR frequency anomalies $+60 \%$ above the mean. We also assess how climate variability influences the IVT magnitude of ARs. However, to accurately capture changes in AR intensity, a more appropriate intensity index (rather than mean IVT magnitude/landfall IVT magnitude) is suggested, such as the category- 5 scale of Ralph et al. (2019). Overall, AR frequency anomalies correlated well with previously understood rainfall patterns for New Zealand. This highlights the potential use of the AR framework to investigate climate variability in New Zealand.

The SAM is identified as the dominant driver of AR activity, though tropical variability also appears to play a key role in driving AR occurrence (particularly for the North Island). Although predictability of the SAM beyond 1-2 weeks is reqarded as low, recent research has shown some potential for seasonal predictions of the SAM, due to association with ENSO and the Antarctic stratospheric circulation (Lim et al., 2013; Seviour et al., 2014). Recent development of coupled ocean-atmosphere models has allowed ENSO to become the most predictable climate mode on monthly to seasonal time scales (Tang et al., 2018). We identified useful relationships between AR frequency and the phase of ENSO, IPO and IOD, suggesting potential for their use in long-range AR forecasts. We also demonstrate the importance of the MJO in modulating AR activity in New Zealand. The $\mathrm{MJO}$ is predictable at subseasonal time-scales, and prediction skill continues to increase. For example, ECMWF re-forecasts have shown improvement in MJO prediction skill equivalent to one day of predictability per year since 2002 (Vitart, 2014). With improved predictions, there is a corresponding improvement in the ability to accurately simulate realistic MJO teleconnections and aspects of midlatitude weather (Vitart, 2014). The analysis in this chapter demonstrates that knowledge of the SAM, ENSO/IPO, IOD and MJO phase may help provide skilful predictions of landfalling ARs in New Zealand on subseasonal to decadal time scales. Skilful long-range predictions of ARs would benefit a number of different sectors such as agriculture, water resource management, energy production, insurance and natural hazard management. 


\section{Chapter 5}

\section{Conclusions}

\section{The Value of AR Forecasts}

As ARs provide both beneficial and hazardous precipitation, forecasting local AR impacts is important for both water management and flood control. However, accurate forecasting of ARs is limited beyond a few days; ARs have a complex and dynamic life-cycle, making forecasting of AR landfall location and intensity difficult. ARs interact with other moisture sources, intensify or weaken, warm or cool, progress rapidly or stall in place, and can merge with other ARs. Predicting AR impacts at landfall is further complicated by the topography, local atmospheric dynamics and precipitation processes. Despite these difficulties, there is significant value in dedicated AR forecasts, particularly at the subseasonal-to-seasonal time-scale. ARs (and IVT) are associated with synoptic-scale atmospheric processes and are therefore expected to have a higher predictability than precipitation, which is more tied to local small-scale processes. By evaluating ensemble forecasts across Europe and the western United States, Lavers et al. (2014) and Lavers et al. (2016) confirmed that IVT has higher predictability than precipitation in these regions. These studies also show that NWP forecasts of synopticscale IVT distributions are skilful at lead times of 7-9 days. Thus, accurate IVT forecasts may help in mitigating and planning for extreme hydrological events at longer lead times.

During the early 21st century, AR research has focused on how to utilize weather model and observations of water vapour transport to more accurately forecast extreme rainfall events (Ralph et al., 2017). In the western United States, a number of AR forecast products have been developed, primarily to serve the reservoir management and hydroelectric power generation sectors (Ralph et al., 2017). For example, the National Centres for Environmental Prediction (NCEP) provides forecasts of water vapour flux at lead times up to seven days. These forecasts are aided by high-resolution mesoscale models (West-WRF), and an observing network consisting of ground-based remote sensing and in situ instruments to better 
monitor AR forcings and impacts (White et al., 2013; Martin et al., 2018). There has been significant investment in AR research in the western United States, with establishment of the CalWater program; an organised effort to better understand water supply, flooding and drought in the region. The CalWater program instituted an AR observing network and AR forecast products which can be accessed through the 'AR portal', https: / ps l noaa.gov/arportal / (Cordeira et al., 2017).

The AR framework has the potential to aid forecasting of extreme weather in New Zealand. At the very least, the term may be useful in communicating extreme weather risk to the public (the term 'Category $5 \mathrm{AR}^{\prime}$ may be interpreted as posing notable risk of extreme weather, joining terms such as 'tropical cyclone' and 'weather bomb'). In communication of extreme weather probability and likely impacts, the AR framework may be particularly effective. For example, in the western United States, forecast products (based on the AR Scale developed by Ralph et al. (2019)) are able to quantify the likelihood, timing, and strength (weak, moderate, extreme) of AR conditions. These products include experimental subseasonal forecasts of AR occurrence, providing probabilistic forecasts of AR landfall at lead times of 1-3 weeks (calculated from ensemble members).

As well as providing severe weather warnings for New Zealand, Metservice ${ }^{1}$ provides monthly outlook forecasts primarily targeted toward New Zealand's rural sector. The National Institute of Water and Atmospheric Research (NIWA) also provides seasonal climate outlooks, providing predictions of air temperature, rainfall, soil moisture and river flow. These forecasts provide a review of current trends in New Zealand's weather systems, including the expected influence of climate modes such as the SAM and ENSO. Recently, the AR science community has emphasized research related to subseasonal-to-seasonal predictions of AR occurrence (DeFlorio et al., 2019). Unlike short-term operational weather forecasts, which are dependent on global numerical weather model predictions, long-range climate forecasts rely on a statistical assessment of historical climate patterns. Therefore, an understanding of the large-scale modes of climate variability is essential. The results presented in this thesis provide a basis for understanding the drivers of AR occurrence in New Zealand. Eventually, the AR framework may be applied in accurate subseasonal-to-seasonal forecasts in New Zealand.

\section{Future Research}

The local hydrological impacts of landfalling ARs is determined by their interaction with the land surface, including terrain height and orientation. These mechanisms can translate the extreme atmospheric moisture flux associated with ARs

\footnotetext{
${ }^{1}$ New Zealand's designated National Meteorological Service
} 
into damaging hydrological impacts (Neiman et al., 2011; Lavers et al., 2012). Therefore, it is essential that weather and climate models accurately represent AR dynamics, thermodynamics, and hydrodynamics. Current global forecast products typically operate at $9 \mathrm{~km}$ spatial resolution for deterministic forecasts and $18 \mathrm{~km}$ for ensemble forecasts. This resolution is not fine enough to accurately resolve the interaction of ARs and complex terrain. Therefore, an assessment of local-scale AR dynamics using high-resolution mesoscale models is suggested as an avenue of future work (likely involving collaboration between the AR science and hydrology fields). Although the 'precipitation-to-discharge' relationship in New Zealand river basins is relatively well understood (Caruso et al., 2013), there is potential for detailed modelling of ARs and their interaction with the topography to significantly improve flood forecasting. Such interdisciplinary programs have proved successful, for example, in the western United States where AR forecasts inform reservoir operations (Cordeira et al., 2017). Due to the importance of fine-scale AR structures in driving extreme local precipitation events, more research is required in determining the mesoscale dynamics of New Zealand ARs, particularly in the Southern Alps. Knowledge of how ARs drive extreme rainfall may improve operational forecasts of severe weather in New Zealand. This is essential, as extreme rainfall events are expected to increase in frequency and intensity under climate change.

The fact that anthropogenic emissions have warmed the global climate is all but certain. Extensive scientific research demonstrates that the observed increase in global mean temperatures since the mid-twentieth century is largely the result of human activities (Intergovernmental Panel on Climate Change, 2014). In a warmer climate, the atmospheric water vapour content is likely to increase; the atmosphere is able to hold approximately $7 \%$ more moisture for every $1^{\circ} \mathrm{C}$ increase in temperature according to the Clausius-Clapeyron equation. Dynamic changes in the circulation patterns transporting moisture around the globe are also expected under a warming climate. Together, these mechanisms are expected to alter the frequency, location and intensity of ARs. Additional research is required to understand climate change impacts on ARs at the regional level. Research objectives set out by the IPCC highlight the need for improved understanding of regional impacts, with a focus on extreme climate and weather events (Intergovernmental Panel on Climate Change, 2014). The AR framework provides an additional tool in determining and predicting climate change impacts for New Zealand. Due to the importance of this research area for policy makers, assessing climate model projections of AR activity in New Zealand should remain a priority.

Though the term and body of literature is relatively new, AR science has benefited a number of sectors including environmental research, flood hazard management, civil engineering, hydroelectricity, and water resource management. Due to growing interest in the field (in both technical and applied sectors), 'at- 
mospheric river' science is expected to continue growing. The emerging field of AR research in New Zealand provides exciting opportunities to improve understanding of extreme weather events, with applications in extreme weather forecasting and freshwater management. The ultimate long-term goal is to bring AR research in New Zealand up to speed with other highly-impacted regions. A number of important research questions remain unanswered; namely, an understanding of the local-scale drivers of AR-related extreme precipitation, and the projected changes in AR frequency and intensity under climate change.

\section{Summary}

The results in this thesis outline the spatial and temporal variability of ARs and AR-related impacts in New Zealand. In particular, we quantify relations between various modes of climate variability and AR occurrence, adding to our understanding of extreme rainfall events in New Zealand.

In chapter 2, we investigate the general climatology of ARs in New Zealand. A prominent seasonal cycle is observed, driven by seasonal changes in the position of the polar and sub-tropical jet streams. In the western South Island, ARs occur most often during the warmer months, with $58 \%$ of all ARs occurring during spring and summer. In the North Island, ARs make landfall most often in winter (between $30 \%$ and $34 \%$ of all ARs) when the subtropical jet is strongest. Increased atmospheric moisture due to higher mean atmospheric temperatures also appears to be a dominant driver of summer AR occurrence. In fact, we find that IVT likely underestimates the impacts of winter ARs in New Zealand. In chapter 3, we confirm the importance of ARs to New Zealand rainfall, particularly extreme events. For northern and western regions, over $45 \%$ of rainfall fell directly under AR conditions, contributing to daily rainfall totals 2.5 times higher on average compared to non-AR days. Further, we found that AR days were associated with up to $70 \%$ of daily rainfall totals above the 99 th percentile. Overall, insurance damages associated with AR events has exceeded NZ \$1.4 billion since 1980. Finally, the large-scale climate drivers of AR occurrence were investigated. The results demonstrate that knowledge of the SAM, ENSO/IPO, IOD and MJO phase may help provide skilful predictions of landfalling ARs in New Zealand on intraseasonal to decadal time scales. 


\section{Bibliography}

Albano, C. M., Dettinger, M. D., and Harpold, A. A. (2020). Patterns and Drivers of Atmospheric River Precipitation and Hydrologic Impacts across the Western United States. Journal of Hydrometeorology, 21(1):143-159.

Algarra, I., Nieto, R., Ramos, A. M., Eiras-Barca, J., Trigo, R. M., and Gimeno, L. (2020). Significant increase of global anomalous moisture uptake feeding landfalling Atmospheric Rivers. Nature Communications, 11(1):1-7.

American Meteorological Society (2021). Atmospheric river. Glossary of Meteorology. http://glossary.ametsoc.org/wiki/Atmospheric_river.

Ashok, K., Nakamura, H., and Yamagata, T. (2007). Impacts of ENSO and Indian Ocean Dipole Events on the Southern Hemisphere Storm-Track Activity during Austral Winter. Journal of Climate, 20(13):3147-3163.

Ault, A. P., Williams, C. R., White, A. B., Neiman, P. J., Creamean, J. M., Gaston, C. J., Ralph, F. M., and Prather, K. A. (2011). Detection of Asian dust in California orographic precipitation. Journal of Geophysical Research, 116(D16):D16205.

Azad, R. and Sorteberg, A. (2017). Extreme daily precipitation in coastal western Norway and the link to atmospheric rivers. Journal of Geophysical Research: Atmospheres, 122(4):2080-2095.

Baggett, C. F., Barnes, E. A., Maloney, E. D., and Mundhenk, B. D. (2017). Advancing atmospheric river forecasts into subseasonal-to-seasonal time scales. Geophysical Research Letters, 44(14):7528-7536.

Bao, J.-W., Michelson, S. A., Neiman, P. J., Ralph, F. M., and Wilczak, J. M. (2006). Interpretation of Enhanced Integrated Water Vapor Bands Associated with Extratropical Cyclones: Their Formation and Connection to Tropical Moisture. Monthly Weather Review, 134(4):1063-1080.

Barry, R. (2005). Synoptic Climatology. In Encyclopedia of World Climatology. Springer. 
Bell, R. G., Lawrence, J. H., Allan, S., Blackett, P., Stephens, S., New Zealand, and Ministry for the Environment (2017). Coastal hazards and climate change: guidance for local government. Ministry for the Environment. OCLC: 1031171543.

Benedict, J. J., Clement, A. C., and Medeiros, B. (2019). Atmospheric Blocking and Other Large-Scale Precursor Patterns of Landfalling Atmospheric Rivers in the North Pacific: A CESM2 Study. Journal of Geophysical Research: Atmospheres, 124(21):11330-11353.

Berbery, E. H. and Vera, C. S. (1996). Characteristics of the Southern Hemisphere Winter Storm Track with Filtered and Unfiltered Data. Journal of the Atmospheric Sciences, 53(3):468-481.

Bjerknes, J. (1966). A possible response of the atmospheric Hadley circulation to equatorial anomalies of ocean temperature. Tellus, 18(4):820-829.

Blamey, R. C., Ramos, A. M., Trigo, R. M., Tomé, R., and Reason, C. J. C. (2018). The Influence of Atmospheric Rivers over the South Atlantic on Winter Rainfall in South Africa. Journal of Hydrometeorology, 19(1):127-142.

Brands, S., Gutiérrez, J. M., and San-Martín, D. (2017). Twentieth-century atmospheric river activity along the west coasts of Europe and North America: algorithm formulation, reanalysis uncertainty and links to atmospheric circulation patterns. Climate Dynamics, 48(9-10):2771-2795.

Branstator, G. (2014). Long-Lived Response of the Midlatitude Circulation and Storm Tracks to Pulses of Tropical Heating. Journal of Climate, 27(23):8809-8826.

Browning, K. A. and Pardoe, C. W. (1973). Structure of low-level jet streams ahead of mid-latitude cold fronts. Quarterly Journal of the Royal Meteorological Society, 99(422):619-638.

Carlson, T. N. (1980). Airflow Through Midlatitude Cyclones and the Comma Cloud Pattern. Monthly Weather Review, 108(10):1498-1509.

Caruso, B. S., Rademaker, M., Balme, A., and Cochrane, T. A. (2013). Flood modelling in a high country mountain catchment, New Zealand: comparing statistical and deterministic model estimates for ecological flows. Hydrological Sciences Journal, 58(2):328-341.

Chang, E. K. M. (1999). Characteristics of Wave Packets in the Upper Troposphere. Part II: Seasonal and Hemispheric Variations. Journal of the Atmospheric Sciences, 56(11):1729-1747.

Clark, A., Mullan, A. B., and Porteous, A. (2011). Scenarios of Regional Drought Under Climate Change. NIWA, page 135. 
Clem, K. R., Renwick, J. A., McGregor, J., and Fogt, R. L. (2016). The relative influence of ENSO and SAM on Antarctic Peninsula climate. Journal of Geophysical Research: Atmospheres, 121(16):9324-9341.

Conway, J. P. and Cullen, N. J. (2016). Cloud effects on surface energy and mass balance in the ablation area of Brewster Glacier, New Zealand. The Cryosphere, 10(1):313-328.

Cordeira, J. M., Ralph, F. M., Martin, A., Gaggini, N., Spackman, J. R., Neiman, P. J., Rutz, J. J., and Pierce, R. (2017). Forecasting Atmospheric Rivers during CalWater 2015. Bulletin of the American Meteorological Society, 98(3):449-459.

Cordeira, J. M., Ralph, F. M., and Moore, B. J. (2013). The Development and Evolution of Two Atmospheric Rivers in Proximity to Western North Pacific Tropical Cyclones in October 2010. Monthly Weather Review, 141(12):4234-4255.

Corringham, T. W., Ralph, F. M., Gershunov, A., Cayan, D. R., and Talbot, C. A. (2019). Atmospheric rivers drive flood damages in the western United States. Science Advances, 5(12).

Cullen, N. J., Gibson, P. B., Mölg, T., Conway, J. P., Sirguey, P., and Kingston, D. G. (2019). The Influence of Weather Systems in Controlling Mass Balance in the Southern Alps of New Zealand. Journal of Geophysical Research: Atmospheres, 124(8):4514-4529.

Dacre, H. F., Clark, P. A., Martinez-Alvarado, O., Stringer, M. A., and Lavers, D. A. (2015). How Do Atmospheric Rivers Form? Bulletin of the American Meteorological Society, 96(8):1243-1255.

Dacre, H. F., Martínez-Alvarado, O., and Mbengue, C. O. (2019). Linking Atmospheric Rivers and Warm Conveyor Belt Airflows. Journal of Hydrometeorology, 20(6):1183-1196.

Dean, S., Rosier, S., Carey-Smith, T., and Stott, P. (2013). The role of climate change in the two-day extreme rainfall in Golden Bay, New Zealand, December 2011. Bulletin of the American Meteorological Society, 94(9):61-63.

Dee, D. P., Uppala, S. M., Simmons, A. J., Berrisford, P., Poli, P., Kobayashi, S., Andrae, U., Balmaseda, M. A., Balsamo, G., Bauer, P., Bechtold, P., Beljaars, A. C. M., Berg, L. v. d., Bidlot, J., Bormann, N., Delsol, C., Dragani, R., Fuentes, M., Geer, A. J., Haimberger, L., Healy, S. B., Hersbach, H., Hólm, E. V., Isaksen, L., Kållberg, P., Köhler, M., Matricardi, M., McNally, A. P., Monge-Sanz, B. M., Morcrette, J.-J., Park, B.-K., Peubey, C., Rosnay, P. d., Tavolato, C., Thépaut, J.-N., and Vitart, F. (2011). The ERA-Interim reanalysis: configuration and performance of the data assimilation system. Quarterly Journal of the Royal Meteorological Society, 137(656):553-597. 
DeFlorio, M. J., Waliser, D. E., Guan, B., Ralph, F. M., and Vitart, F. (2019). Global evaluation of atmospheric river subseasonal prediction skill. Climate Dynamics, 52(5-6):3039-3060.

Dessler, A. E., Zhang, Z., and Yang, P. (2008). Water-vapor climate feedback inferred from climate fluctuations, 2003-2008. Geophysical Research Letters, 35(20).

Dettinger, M. D., Ralph, F. M., Das, T., Neiman, P. J., and Cayan, D. R. (2011). Atmospheric Rivers, Floods and the Water Resources of California. Water, 3(2):445-478.

Diamond, H. J., Lorrey, A. M., and Renwick, J. A. (2013). A Southwest Pacific Tropical Cyclone Climatology and Linkages to the El Niño-Southern Oscillation. Journal of Climate, 26(1):3-25.

Dravitzki, S. and McGregor, J. (2011). Extreme precipitation of the Waikato region, New Zealand. International Journal of Climatology, 31(12):1803-1812.

Eichler, T. P. and Gottschalck, J. (2013). A Comparison of Southern Hemisphere Cyclone Track Climatology and Interannual Variability in Coarse-Gridded Reanalysis Datasets. Advances in Meteorology, 2013.

Esfandiari, N. and Lashkari, H. (2020). Identifying atmospheric river events and their paths into Iran. Theoretical and Applied Climatology, 140(3-4):1125-1137.

Espinoza, V., Waliser, D. E., Guan, B., Lavers, D. A., and Ralph, F. M. (2018). Global Analysis of Climate Change Projection Effects on Atmospheric Rivers. Geophysical Research Letters, 45(9):4299-4308.

Fauchereau, N., Pohl, B., and Lorrey, A. (2016). Extratropical Impacts of the Madden-Julian Oscillation over New Zealand from a Weather Regime Perspective. Journal of Climate, 29(6):2161-2175.

Fitzharris, B. B., Chinn, T. J., and Lamont, G. N. (1997). Glacier balance fluctuations and atmospheric circulation patterns over the Southern Alps, New Zealand. International Journal of Climatology, 17(7):745-763.

Flatau, M. and Kim, Y.-J. (2013). Interaction between the MJO and Polar Circulations. Journal of Climate, 26(11):3562-3574.

Fogt, R. L., Jones, J. M., and Renwick, J. (2012). Seasonal Zonal Asymmetries in the Southern Annular Mode and Their Impact on Regional Temperature Anomalies. Journal of Climate, 25(18):6253-6270.

Fogt, R. L. and Marshall, G. J. (2020). The Southern Annular Mode: Variability, trends, and climate impacts across the Southern Hemisphere. WIREs Climate Change, 11(4). 
Frame, D. J., Rosier, S. M., Noy, I., Harrington, L. J., Carey-Smith, T., Sparrow, S. N., Stone, D. A., and Dean, S. M. (2020). Climate change attribution and the economic costs of extreme weather events: a study on damages from extreme rainfall and drought. Climatic Change, 162(2):781-797.

Gillett, N. P. and Thompson, D. W. J. (2003). Simulation of Recent Southern Hemisphere Climate Change. Science, 302(5643):273-275.

Gimeno, L., Nieto, R., Vázquez, M., and Lavers, D. A. (2014). Atmospheric rivers: a mini-review. Frontiers in Earth Science, 2.

Gonzales, K. R., Swain, D. L., Barnes, E. A., and Diffenbaugh, N. S. (2020). Moisture- Versus Wind-Dominated Flavors of Atmospheric Rivers. Geophysical Research Letters, 47(23):e2020GL090042.

Gordon, N. D. (1986). The Southern Oscillation and New Zealand Weather. Monthly Weather Review, 114(2):371-387.

Griffiths (2007). Changes in New Zealand daily rainfall extremes 1930 - 2004. Weather and Climate, 27:3.

Griffiths (2011). Drivers of extreme daily rainfalls in New Zealand. Weather and Climate, 31:24.

Guan, B., Molotch, N. P., Waliser, D. E., Fetzer, E. J., and Neiman, P. J. (2013). The 2010/2011 snow season in California's Sierra Nevada: Role of atmospheric rivers and modes of large-scale variability. Water Resources Research, 49(10):6731-6743.

Guan, B. and Waliser, D. E. (2015). Detection of atmospheric rivers: Evaluation and application of an algorithm for global studies: Detection of Atmospheric Rivers. Journal of Geophysical Research: Atmospheres, 120(24):12514-12535.

Guan, B. and Waliser, D. E. (2019). Tracking Atmospheric Rivers Globally: Spatial Distributions and Temporal Evolution of Life Cycle Characteristics. Journal of Geophysical Research: Atmospheres, 124(23):12523-12552.

Guan, B., Waliser, D. E., Molotch, N. P., Fetzer, E. J., and Neiman, P. J. (2012). Does the Madden-Julian Oscillation Influence Wintertime Atmospheric Rivers and Snowpack in the Sierra Nevada? Monthly Weather Review, 140(2):325-342.

Guan, B., Waliser, D. E., and Ralph, F. M. (2018). An Intercomparison between Reanalysis and Dropsonde Observations of the Total Water Vapor Transport in Individual Atmospheric Rivers. Journal of Hydrometeorology, 19(2):321-337.

Guildford, J. (2019). West Coast's Waiho River bridge to close at night as final construction begins. The Press, 2 May, p.3. 
Hatchett, B. J., Burak, S., Rutz, J. J., Oakley, N. S., Bair, E. H., and Kaplan, M. L. (2017). Avalanche Fatalities during Atmospheric River Events in the Western United States. Journal of Hydrometeorology, 18(5):1359-1374.

Held, I. M. (1993). Large-Scale Dynamics and Global Warming. Bulletin of the American Meteorological Society, 74(2):228-242.

Henderson, R. D. and Thompson, S. M. (1999). Extreme Rainfalls in the Southern Alps of New Zealand. Journal of Hydrology (New Zealand), 38(2):309-330.

Hendon, H. H., Wheeler, M. C., and Zhang, C. (2007). Seasonal Dependence of the MJO-ENSO Relationship. Journal of Climate, 20(3):531-543.

Henley, B. J., Gergis, J., Karoly, D. J., Power, S., Kennedy, J., and Folland, C. K. (2015). A Tripole Index for the Interdecadal Pacific Oscillation. Climate Dynamics, 45(11):3077-3090.

Hersbach, H., Bell, B., Berrisford, P., Hirahara, S., Horányi, A., Muñoz-Sabater, J., Nicolas, J., Peubey, C., Radu, R., Schepers, D., Simmons, A., Soci, C., Abdalla, S., Abellan, X., Balsamo, G., Bechtold, P., Biavati, G., Bidlot, J., Bonavita, M., Chiara, G., Dahlgren, P., Dee, D., Diamantakis, M., Dragani, R., Flemming, J., Forbes, R., Fuentes, M., Geer, A., Haimberger, L., Healy, S., Hogan, R. J., Hólm, E., Janisková, M., Keeley, S., Laloyaux, P., Lopez, P., Lupu, C., Radnoti, G., Rosnay, P., Rozum, I., Vamborg, F., Villaume, S., and Thépaut, J. (2020). The ERA5 global reanalysis. Quarterly Journal of the Royal Meteorological Society, 146(730):1999-2049.

Holton, J. (2004). An Introduction to Dynamic Meteorology. Academic Press, 4 edition.

Hoskins, B. J. and Hodges, K. I. (2005). A New Perspective on Southern Hemisphere Storm Tracks. Journal of Climate, 18(20):4108-4129.

Iacono, M. J., Delamere, J. S., Mlawer, E. J., Shephard, M. W., Clough, S. A., and Collins, W. D. (2008). Radiative forcing by long-lived greenhouse gases: Calculations with the AER radiative transfer models. Journal of Geophysical Research, 113(D13):D13103.

Intergovernmental Panel on Climate Change (2014). Detection and Attribution of Climate Change: from Global to Regional. In Climate Change 2013 - The Physical Science Basis, pages 867-952. Cambridge University Press, Cambridge.

Jiang, N. (2011). A new objective procedure for classifying New Zealand synoptic weather types during 1958-2008. International Journal of Climatology, 31(6):863879. 
Kain, J. S. (2004). The Kain-Fritsch Convective Parameterization: An Update. JOURNAL OF APPLIED METEOROLOGY, 43:12.

Kamae, Y., Mei, W., Xie, S.-P., Naoi, M., and Ueda, H. (2017). Atmospheric Rivers over the Northwestern Pacific: Climatology and Interannual Variability. Journal of Climate, 30(15):5605-5619.

Kennett, D. (2020). New Zealand Detection Algorithm. Available upon request from the author.

Kennett, D. (2021). Atmospheric river detection algorithm, kennett (2021).

Kerr, T., Owens, I., and Henderson, R. (2011). The precipitation distribution in the Lake Pukaki Catchment. Journal of Hydrology (New Zealand), 50.

Kidson, J. W. (2000). An analysis of New Zealand synoptic types and their use in defining weather regimes. Int. J. Climatol., page 18.

Kidson, J. W. and Renwick, J. A. (2002). Patterns of convection in the tropical pacific and their influence on New Zealand weather. International Journal of Climatology, 22(2):151-174.

Kidston, J., Renwick, J. A., and McGregor, J. (2009). Hemispheric-Scale Seasonality of the Southern Annular Mode and Impacts on the Climate of New Zealand. Journal of Climate, 22(18):4759-4770.

Kingston, D. G., Lavers, D. A., and Hannah, D. M. (2016). Floods in the Southern Alps of New Zealand: the importance of atmospheric rivers: Atmospheric Rivers and Floods in New Zealand. Hydrological Processes, 30(26):5063-5070.

Lavers, D. A., Allan, R. P., Wood, E. F., Villarini, G., Brayshaw, D. J., and Wade, A. J. (2011). Winter floods in Britain are connected to atmospheric rivers: UK Winter Floods and Atmospheric Rivers. Geophysical Research Letters, 38(23).

Lavers, D. A., Pappenberger, F., and Zsoter, E. (2014). Extending medium-range predictability of extreme hydrological events in Europe. Nature Communications, 5(1):5382.

Lavers, D. A., Ralph, F. M., Richardson, D. S., and Pappenberger, F. (2020). Improved forecasts of atmospheric rivers through systematic reconnaissance, better modelling, and insights on conversion of rain to flooding. Communications Earth E Environment, 1(1):1-7. Number: 1 Publisher: Nature Publishing Group.

Lavers, D. A. and Villarini, G. (2013). The nexus between atmospheric rivers and extreme precipitation across Europe: ARs and Extreme European Precipitation. Geophysical Research Letters, 40(12):3259-3264. 
Lavers, D. A., Villarini, G., Allan, R. P., Wood, E. F., and Wade, A. J. (2012). The detection of atmospheric rivers in atmospheric reanalyses and their links to British winter floods and the large-scale climatic circulation: ARs and British Winter Floods. Journal of Geophysical Research: Atmospheres, 117(D20).

Lavers, D. A., Waliser, D. E., Ralph, F. M., and Dettinger, M. D. (2016). Predictability of horizontal water vapor transport relative to precipitation: Enhancing situational awareness for forecasting western U.S. extreme precipitation and flooding. Geophysical Research Letters, 43(5):2275-2282.

Lee, S. and Held, I. M. (1993). Baroclinic Wave Packets in Models and Observations. Journal of the Atmospheric Sciences, 50(10):1413-1428.

Lim, E.-P., Hendon, H. H., and Rashid, H. (2013). Seasonal Predictability of the Southern Annular Mode due to Its Association with ENSO. Journal of Climate, 26(20):8037-8054.

Little, K., Kingston, D. G., Cullen, N. J., and Gibson, P. B. (2019). The Role of Atmospheric Rivers for Extreme Ablation and Snowfall Events in the Southern Alps of New Zealand. Geophysical Research Letters, 46(5):2761-2771.

Lorrey, A., Fauchereau, N., Stanton, C., Chappell, P., Phipps, S., Mackintosh, A., Renwick, J., Goodwin, I., and Fowler, A. (2014a). The Little Ice Age climate of New Zealand reconstructed from Southern Alps cirque glaciers: a synoptic type approach. Climate Dynamics, 42(11):3039-3060.

Lorrey, A., Fowler, A. M., and Salinger, J. (2007). Regional climate regime classification as a qualitative tool for interpreting multi-proxy palaeoclimate data spatial patterns: A New Zealand case study. Palaeogeography, Palaeoclimatology, Palaeoecology, 253(3):407-433.

Lorrey, A. M., Griffiths, G., Fauchereau, N., Diamond, H. J., Chappell, P. R., and Renwick, J. (2014b). An ex-tropical cyclone climatology for Auckland, New Zealand. International Journal of Climatology, 34(4):1157-1168.

Ma, W., Chen, G., and Guan, B. (2020). Poleward Shift of Atmospheric Rivers in the Southern Hemisphere in Recent Decades. Geophysical Research Letters, 47(21):e2020GL089934.

Madden, R. A. and Julian, P. R. (1971). Detection of a 40-50 Day Oscillation in the Zonal Wind in the Tropical Pacific. Journal of the Atmospheric Sciences, 28(5):702708.

Mantua, N. J., Hare, S. R., Zhang, Y., Wallace, J. M., and Francis, R. C. (1997). A Pacific Interdecadal Climate Oscillation with Impacts on Salmon Production. Bulletin of the American Meteorological Society, 78(6):11. 
Marshall, G. J. (2003). Trends in the Southern Annular Mode from Observations and Reanalyses. Journal of Climate, 16:10.

Martin, A., Ralph, F. M., Demirdjian, R., DeHaan, L., Weihs, R., Helly, J., Reynolds, D., and Iacobellis, S. (2018). Evaluation of Atmospheric River Predictions by the WRF Model Using Aircraft and Regional Mesonet Observations of Orographic Precipitation and Its Forcing. Journal of Hydrometeorology, 19(7):1097-1113.

Matthews, A. J. and Meredith, M. P. (2004). Variability of Antarctic circumpolar transport and the Southern Annular Mode associated with the Madden-Julian Oscillation. Geophysical Research Letters, 31(24).

Matthews, A. J., Singhruck, P., and Heywood, K. J. (2010). Ocean temperature and salinity components of the Madden-Julian oscillation observed by Argo floats. Climate Dynamics, 35(7):1149-1168.

McKerchar, A. I. and Pearson, C. P. (2001). Factors causing flooding to be New Zealand's number one hazard. Tephra, Feb 2001; v.18:p.9-15.

McKerchar, A. I., Renwick, J. A., and Schmidt, J. (2010). Diminishing streamflows on the east coast of the South Island New Zealand and linkage to climate variability and change. Journal of Hydrology (New Zealand), 49(1):1-14.

McPhaden, M. J. (1999). Genesis and Evolution of the 1997-98 El Nino. Science, 283(5404):950-954.

McPhaden, M. J., Zebiak, S. E., and Glantz, M. H. (2006). ENSO as an Integrating Concept in Earth Science. Science, 314(5806):1740-1745.

Mosley, M. P. (2000). Regional differences in the effects of El Niño and La Niña on low flows and floods. Hydrological Sciences Journal, 45(2):249-267.

Mullan, A. B. (1995). On the linearity and stability of Southern Oscillation-climate relationships for New Zealand. International Journal of Climatology, 15(12):13651386.

Mullan, A. B. (1998). Southern hemisphere sea-surface temperatures and their contemporary and lag association with New Zealand temperature and precipitation. International Journal of Climatology, 18(8):817-840.

Mundhenk, B. D., Barnes, E. A., and Maloney, E. D. (2016). All-Season Climatology and Variability of Atmospheric River Frequencies over the North Pacific. Journal of Climate, 29(13):4885-4903. 
Mundhenk, B. D., Barnes, E. A., Maloney, E. D., and Baggett, C. F. (2018). Skillful empirical subseasonal prediction of landfalling atmospheric river activity using the Madden-Julian oscillation and quasi-biennial oscillation. npj Climate and Atmospheric Science, 1(1):20177.

Muszynski, G., Kashinath, K., Kurlin, V., Wehner, M., and Prabhat (2019). Topological data analysis and machine learning for recognizing atmospheric river patterns in large climate datasets. Geoscientific Model Development, 12(2):613628.

Nakamura, H. and Shimpo, A. (2004). Seasonal Variations in the Southern Hemisphere Storm Tracks and Jet Streams as Revealed in a Reanalysis Dataset. Journal of Climate, 17(9):1828-1844.

Nakanishi, M. and Niino, H. (2006). An Improved Mellor-Yamada Level-3 Model: Its Numerical Stability and Application to a Regional Prediction of Advection Fog. Boundary-Layer Meteorology, 119(2):397-407.

Nayak, M. A. and Villarini, G. (2017). A long-term perspective of the hydroclimatological impacts of atmospheric rivers over the central United States. Water Resources Research, 53(2):1144-1166.

Nayak, M. A., Villarini, G., and Lavers, D. A. (2014). On the skill of numerical weather prediction models to forecast atmospheric rivers over the central United States. Geophysical Research Letters, 41(12):4354-4362.

Neiman, P. J., Ralph, F. M., Wick, G. A., Lundquist, J. D., and Dettinger, M. D. (2008). Meteorological Characteristics and Overland Precipitation Impacts of Atmospheric Rivers Affecting the West Coast of North America Based on Eight Years of SSM/I Satellite Observations. Journal of Hydrometeorology, 9(1):22-47.

Neiman, P. J., Schick, L. J., Ralph, F. M., Hughes, M., and Wick, G. A. (2011). Flooding in Western Washington: The Connection to Atmospheric Rivers. Journal of Hydrometeorology, 12(6):1337-1358.

Newell, R. E., Newell, N. E., Zhu, Y., and Scott, C. (1992). Tropospheric rivers? A pilot study. Geophysical Research Letters, 19(24):2401-2404.

Nicolas, J. P., Vogelmann, A. M., Scott, R. C., Wilson, A. B., Cadeddu, M. P., Bromwich, D. H., Verlinde, J., Lubin, D., Russell, L. M., Jenkinson, C., Powers, H. H., Ryczek, M., Stone, G., and Wille, J. D. (2017). January 2016 extensive summer melt in West Antarctica favoured by strong El Niño. Nature Communications, 8(1):15799.

Niu, G.-Y., Yang, Z.-L., Mitchell, K. E., Chen, F., Ek, M. B., Barlage, M., Kumar, A., Manning, K., Niyogi, D., Rosero, E., Tewari, M., and Xia, Y. (2011). The community Noah land surface model with multiparameterization options (Noah-MP): 
1. Model description and evaluation with local-scale measurements. Journal of Geophysical Research, 116(D12):D12109.

Oakley, N. S., Lancaster, J. T., Kaplan, M. L., and Ralph, F. M. (2017). Synoptic conditions associated with cool season post-fire debris flows in the Transverse Ranges of southern California. Natural Hazards, 88(1):327-354.

Paltan, H., Waliser, D., Lim, W. H., Guan, B., Yamazaki, D., Pant, R., and Dadson, S. (2017). Global Floods and Water Availability Driven by Atmospheric Rivers: Global Hydrology and ARs. Geophysical Research Letters, 44(20):10,387-10,395.

Pan, M. and Lu, M. (2019). A Novel Atmospheric River Identification Algorithm. Water Resources Research, 55(7):6069-6087.

Payne, A. E., Demory, M.-E., Leung, L. R., Ramos, A. M., Shields, C. A., Rutz, J. J., Siler, N., Villarini, G., Hall, A., and Ralph, F. M. (2020). Responses and impacts of atmospheric rivers to climate change. Nature Reviews Earth $\mathcal{E}$ Environment, 1(3):143-157.

Pohl, B., Richard, Y., and Fauchereau, N. (2007). Influence of the Madden-Julian Oscillation on Southern African Summer Rainfall. Journal of Climate, 20(16):4227-4242.

Poole, L. R. and McCormick, M. P. (1988). Polar stratospheric clouds and the Antarctic ozone hole. Journal of Geophysical Research, 93(D7):8423.

Porhemmat, R., Purdie, H., Zawar-Reza, P., Zammit, C., and Kerr, T. (2021). Moisture Transport during Large Snowfall Events in the New Zealand Southern Alps: The Role of Atmospheric Rivers. Journal of Hydrometeorology, 22(2):425444 .

Power, S., Casey, T., Folland, C., Colman, A., and Mehta, V. (1999). Inter-decadal modulation of the impact of ENSO on Australia. Climate Dynamics, 15(5):319324.

Prince, H. D., Cullen, N. J., Gibson, P. B., Conway, J., and Kingston, D. G. (2021). A climatology of atmospheric rivers in New Zealand. Journal of Climate.

Purdie, H., Mackintosh, A., Lawson, W., and Anderson, B. (2011). Synoptic Influences on Snow Accumulation on Glaciers East and West of a Topographic Divide: Southern Alps, New Zealand. Arctic, Antarctic, and Alpine Research, 43(1):82-94.

Rabinowitz, J. L., Lupo, A. R., and Guinan, P. E. (2018). Evaluating Linkages between Atmospheric Blocking Patterns and Heavy Rainfall Events across the North-Central Mississippi River Valley for Different ENSO Phases. Advances in Meteorology, 2018:e1217830. 
Ralph, F. M., Coleman, T., Neiman, P. J., Zamora, R. J., and Dettinger, M. D. (2013). Observed Impacts of Duration and Seasonality of Atmospheric-River Landfalls on Soil Moisture and Runoff in Coastal Northern California. Journal of Hydrometeorology, 14(2):443-459.

Ralph, F. M., Dettinger, M., Lavers, D., Gorodetskaya, I. V., Martin, A., Viale, M., White, A. B., Oakley, N., Rutz, J., Spackman, J. R., Wernli, H., and Cordeira, J. (2017a). Atmospheric Rivers Emerge as a Global Science and Applications Focus. Bulletin of the American Meteorological Society, 98(9):1969-1973.

Ralph, F. M., Iacobellis, S. F., Neiman, P. J., Cordeira, J. M., Spackman, J. R., Waliser, D. E., Wick, G. A., White, A. B., and Fairall, C. (2017b). Dropsonde Observations of Total Integrated Water Vapor Transport within North Pacific Atmospheric Rivers. Journal of Hydrometeorology, 18(9):2577-2596.

Ralph, F. M., Neiman, P. J., Kiladis, G. N., Weickmann, K., and Reynolds, D. W. (2011). A Multiscale Observational Case Study of a Pacific Atmospheric River Exhibiting Tropical-Extratropical Connections and a Mesoscale Frontal Wave. Monthly Weather Review, 139(4):1169-1189.

Ralph, F. M., Neiman, P. J., and Wick, G. A. (2004). Satellite and CALJET Aircraft Observations of Atmospheric Rivers over the Eastern North Pacific Ocean during the Winter of 1997/98. Monthly Weather Review, 132:26.

Ralph, F. M., Neiman, P. J., Wick, G. A., Gutman, S. I., Dettinger, M. D., Cayan, D. R., and White, A. B. (2006). Flooding on California's Russian River: Role of atmospheric rivers. Geophysical Research Letters, 33(13):L13801.

Ralph, F. M., Rutz, J. J., Cordeira, J. M., Dettinger, M., Anderson, M., Reynolds, D., Schick, L. J., and Smallcomb, C. (2019). A Scale to Characterize the Strength and Impacts of Atmospheric Rivers. Bulletin of the American Meteorological Society, 100(2):269-289.

Ramos, A. M., Trigo, R. M., Liberato, M. L. R., and Tomé, R. (2015). Daily Precipitation Extreme Events in the Iberian Peninsula and Its Association with Atmospheric Rivers. Journal of Hydrometeorology, 16(2):579-597.

Ramos, A. M., Trigo, R. M., Tomé, R., and Liberato, M. L. R. (2018). Impacts of Atmospheric Rivers in Extreme Precipitation on the European Macaronesian Islands. Atmosphere, 9(8):325.

Rao, V. B., Carmo, A. M. C. d., and Franchito, S. H. (2002). Seasonal Variations in the Southern Hemisphere Storm Tracks and Associated Wave Propagation. Journal of the Atmospheric Sciences, 59(6):1029-1040. 
Reid, K. J., King, A. D., Lane, T. P., and Short, E. (2020). The Sensitivity of Atmospheric River Identification to Integrated Water Vapor Transport Threshold, Resolution, and Regridding Method. Journal of Geophysical Research: Atmospheres, 125(20):e2020JD032897.

Reid, K. J., Rosier, S. M., Harrington, L. J., King, A. D., and Lane, T. P. (2021). Extreme rainfall in New Zealand and its association with Atmospheric Rivers. Environmental Research Letters, 16(4):044012.

Reisinger, A., Kitching, R., Chiew, F., Hughes, L., Newton, P., Schuster, S., Tait, A., and Whetton, P. (2014). Climate Change 2014: Impacts, Adaptation, and Vulnerability. Part B: Regional Aspects. Contribution of Working Group II to the Fifth Assessment Report of the Intergovernmental Panel on Climate Change.

Renwick, J. A. (2011). Kidson's Synoptic Weather Types and Surface Climate Variability over New Zealand. Weather and Climate, 31:3-23. Publisher: Meteorological Society of New Zealand.

Renwick, J. A. and Thompson, D. (2006). The Southern Annular Mode and New Zealand climate. Water E Atmosphere, 14.

Rosier, S., Dean, S., Stuart, S., Carey-Smith, T., Black, M., and Massey, N. (2015). Extreme rainfall in early July 2014 in Northland, New Zealand-was there an anthropogenic influence? Bulletin of the American Meteorological Society, 96(12):136-140.

Rouse, H. L., GNS Science (N.Z.), and National Institute of Water and Atmospheric Research (N.Z.) (2012). Flood risk management research in New Zealand: where are we, and where are we going? GNS Science. OCLC: 864712787.

Rutz, J. J., Shields, C. A., Lora, J. M., Payne, A. E., Guan, B., Ullrich, P., O’Brien, T., Leung, L. R., Ralph, F. M., Wehner, M., Brands, S., Collow, A., Goldenson, N., Gorodetskaya, I., Griffith, H., Kashinath, K., Kawzenuk, B., Krishnan, H., Kurlin, V., Lavers, D., Magnusdottir, G., Mahoney, K., McClenny, E., Muszynski, G., Nguyen, P. D., Prabhat, M., Qian, Y., Ramos, A. M., Sarangi, C., Sellars, S., Shulgina, T., Tome, R., Waliser, D., Walton, D., Wick, G., Wilson, A. M., and Viale, M. (2019). The Atmospheric River Tracking Method Intercomparison Project (ARTMIP): Quantifying Uncertainties in Atmospheric River Climatology. Journal of Geophysical Research: Atmospheres, 124(24):13777-13802.

Rutz, J. J., Steenburgh, W. J., and Ralph, F. M. (2014). Climatological Characteristics of Atmospheric Rivers and Their Inland Penetration over the Western United States. Monthly Weather Review, 142(2):905-921.

Salinger, M., Renwick, J., and Mullan, A. (2001). Interdecadal Pacific Oscillation and South Pacific climate. International Journal of Climatology, 21(14):1705-1721. 
Salinger, M. J. and Mullan, A. B. (1999). New Zealand climate: temperature and precipitation variations and their links with atmospheric circulation 1930-1994. International Journal of Climatology, 19(10):1049-1071.

Salinger, M. J. and Porteous, A. (2014). New Zealand climate: patterns of drought 1941/42 -2012/13. Weather and Climate, 34.

Sardeshmukh, P. D. and Hoskins, B. J. (1985). Vorticity balances in the tropics during the 1982-83 El Niñio-Southern oscillation event. Quarterly Journal of the Royal Meteorological Society, 111(468):261-278.

Scarsbrook, M. R., McBride, C. G., McBride, G. B., and Bryers, G. G. (2003). Effects of climate variability on rivers: consequences for long term water quality analysis. Journal of the American Water Resources Association, 39(6):1435-1447.

Seviour, W. J. M., Hardiman, S. C., Gray, L. J., Butchart, N., MacLachlan, C., and Scaife, A. A. (2014). Skillful Seasonal Prediction of the Southern Annular Mode and Antarctic Ozone. Journal of Climate, 27(19):7462-7474.

Shepherd, T. G. (2014). Atmospheric circulation as a source of uncertainty in climate change projections. Nature Geoscience, 7(10):703-708.

Shimizu, M. H. and Ambrizzi, T. (2016). MJO influence on ENSO effects in precipitation and temperature over South America. Theoretical and Applied Climatology, 124:291-301.

Shindell, D. T. and Schmidt, G. A. (2004). Southern Hemisphere climate response to ozone changes and greenhouse gas increases. Geophysical Research Letters, 31(18).

Simmonds, I. and Keay, K. (2000). Mean Southern Hemisphere Extratropical Cyclone Behavior in the 40-Year NCEP-NCAR Reanalysis. Journal of Climate, 13(5):873-885.

Sinclair, M. (1995). A Climatology of Cyclogenesis for the Southern Hemisphere. Monthly Weather Review, 123:1601-1619.

Sinclair, M. R. (1996). A Climatology of Anticyclones and Blocking for the Southern Hemisphere. Monthly Weather Review, 124(2):245-264.

Sinclair, M. R. (2002). Extratropical Transition of Southwest Pacific Tropical Cyclones. Part I: Climatology and Mean Structure Changes. Monthly Weather Review, 130:20.

Skamarock, W. C., Klemp, J. B., Dudhia, J., Gill, D. O., Liu, Z., Berner, J., Wang, W., Powers, J. G., Duda, M. G., Barker, D. M., and Huang, X.-Y. (2019). A Description of the Advanced Research WRF Model Version 4. NCAR Tech. 
Smart, G. M. and McKerchar, A. I. (2010). More flood disasters in New Zealand. Journal of Hydrology (NZ), 49(2):69-78.

Solman, S. A. and Orlanski, I. (2014). Poleward Shift and Change of Frontal Activity in the Southern Hemisphere over the Last 40 Years. Journal of the Atmospheric Sciences, 71(2):539-552.

Solomon, S., Ivy, D. J., Kinnison, D., Mills, M. J., Neely, R. R., and Schmidt, A. (2016). Emergence of healing in the Antarctic ozone layer. Science, 353(6296):269-274.

Sturman, A. P. and Tapper, N. J. (2006). The Weather and Climate of Australia and New Zealand. Oxford University Press.

Tait, A. B. and Fitzharris, B. B. (1998). Relationships between New Zealand rainfall and south-west Pacific pressure patterns. Int. J. Climatol., page 18.

Taljaard, J. J. (1972). Synoptic Meteorology of the Southern Hemisphere. In van Loon, H., Taljaard, J. J., Sasamori, T., London, J., Hoyt, D. V., Labitzke, K., Newton, C. W., and Newton, C. W., editors, Meteorology of the Southern Hemisphere, Meteorological Monographs, pages 139-213. American Meteorological Society, Boston, MA.

Tang, Y., Zhang, R.-H., Liu, T., Duan, W., Yang, D., Zheng, F., Ren, H., Lian, T., Gao, C., Chen, D., and Mu, M. (2018). Progress in ENSO prediction and predictability study. National Science Review, 5(6):826-839.

Thompson, D. W. J., Solomon, S., Kushner, P. J., England, M. H., Grise, K. M., and Karoly, D. J. (2011). Signatures of the Antarctic ozone hole in Southern Hemisphere surface climate change. Nature Geoscience, 4(11):741-749.

Thompson, D. W. J. and Wallace, J. M. (2000). Annular Modes in the Extratropical Circulation. Part I: Month-to-Month Variability. Journal of Climate, 13:17.

Thompson, G., Field, P. R., Rasmussen, R. M., and Hall, W. D. (2008). Explicit Forecasts of Winter Precipitation Using an Improved Bulk Microphysics Scheme. Part II: Implementation of a New Snow Parameterization. Monthly Weather Review, 136(12):5095-5115.

Trenberth, K. E. (1991). Storm Tracks in the Southern Hemisphere. Journal of the Atmospheric Sciences, 48(19):2159-2178.

Turner, J. (2004). The El Niño-southern oscillation and Antarctica: ENSO and the Antarctic. International Journal of Climatology, 24(1):1-31.

Ummenhofer, C. C. and England, M. H. (2007). Interannual Extremes in New Zealand Precipitation Linked to Modes of Southern Hemisphere Climate Variability. Journal of Climate, 20(21):5418-5440. 
Viale, M., Valenzuela, R., Garreaud, R. D., and Ralph, F. M. (2018). Impacts of Atmospheric Rivers on Precipitation in Southern South America. Journal of Hydrometeorology, 19(10):1671-1687.

Vitart, F. (2014). Evolution of ECMWF sub-seasonal forecast skill scores: Evolution of the ECMWF Sub-Seasonal Forecast Skill. Quarterly Journal of the Royal Meteorological Society, 140(683):1889-1899.

Vitart, F., Robertson, A. W., and Anderson, D. L. T. (2012). Subseasonal to Seasonal Prediction Project: bridging the gap between weather and climate. ECMWF, page 6.

Waliser, D. E., Moncrieff, M. W., Burridge, D., Fink, A. H., Gochis, D., Goswami, B. N., Guan, B., Harr, P., Heming, J., Hsu, H.-H., Jakob, C., Janiga, M., Johnson, R., Jones, S., Knippertz, P., Marengo, J., Nguyen, H., Pope, M., Serra, Y., Thorncroft, C., Wheeler, M., Wood, R., and Yuter, S. (2012). The "Year" of Tropical Convection (May 2008-April 2010): Climate Variability and Weather Highlights. Bulletin of the American Meteorological Society, 93(8):1189-1218.

Wallace, J. M. and Hobbs, P. (2006). Atmospheric Science, Second Edition: An Introductory Survey. Academic Press, 2 edition.

Wang, S., Xu, T., Nie, W., Jiang, C., Yang, Y., Fang, Z., Li, M., and Zhang, Z. (2020). Evaluation of Precipitable Water Vapor from Five Reanalysis Products with Ground-Based GNSS Observations. Remote Sensing, 12(11):1817.

Wang, X. L. (2008). Accounting for Autocorrelation in Detecting Mean Shifts in Climate Data Series Using the Penalized Maximal $t$ or $F$ Test. Journal of Applied Meteorology and Climatology, 47(9):2423-2444.

Wang, X. L. and Feng, Y. (2013). RHtests_dlyprcp User Manual. Environment Canada. http:/ / etccdi.pacificclimate.org/software.shtml.

Webster, C. S., Kingston, D. G., and Kerr, T. (2015). Inter-annual variation in the topographic controls on catchment-scale snow distribution in a maritime alpine catchment, New Zealand. Hydrological Processes, 29(6):1096-1109.

Wheeler, M. C. and Hendon, H. H. (2004). An All-Season Real-Time Multivariate MJO Index: Development of an Index for Monitoring and Prediction. Monthly Weather Review, 132:16.

White, A. B., Anderson, M. L., Dettinger, M. D., Ralph, F. M., Hinojosa, A., Cayan, D. R., Hartman, R. K., Reynolds, D. W., Johnson, L. E., Schneider, T. L., Cifelli, R., Toth, Z., Gutman, S. I., King, C. W., Gehrke, F., Johnston, P. E., Walls, C., Mann, D., Gottas, D. J., and Coleman, T. (2013). A Twenty-First-Century California Observing Network for Monitoring Extreme Weather Events. Journal of Atmospheric and Oceanic Technology, 30(8):1585-1603. 
Whittaker, L. M. and Horn, L. H. (1984). Northern Hemisphere extratropical cyclone activity for four mid-season months. Journal of Climatology, 4(3):297-310.

Wick, G. A., Neiman, P. J., and Ralph, F. M. (2013). Description and Validation of an Automated Objective Technique for Identification and Characterization of the Integrated Water Vapor Signature of Atmospheric Rivers. IEEE Transactions on Geoscience and Remote Sensing, 51(4):2166-2176.

Yang, D., Arblaster, J. M., Meehl, G. A., England, M. H., Lim, E.-P., Bates, S., and Rosenbloom, N. (2020). Role of Tropical Variability in Driving Decadal Shifts in the Southern Hemisphere Summertime Eddy-Driven Jet. Journal of Climate, 33(13):5445-5463.

Yuan, X., Kaplan, M. R., and Cane, M. A. (2018). The Interconnected Global Climate System-A Review of Tropical-Polar Teleconnections. Journal of Climate, 31(15):5765-5792.

Yuan, Y. and Li, C. (2008). Decadal variability of the IOD-ENSO relationship. Chinese Science Bulletin, 53(11):1745-1752.

Zhang, C. (2005). Madden-Julian Oscillation. Reviews of Geophysics, 43(2).

Zhang, X., Feng, Y., and Chan, R. (2018). User Manual: Introduction to RClimDex v1.9. Environment Canada. http://etccdi.pacificclimate.org/software.shtml.

Zhang, Z., Ralph, F. M., and Zheng, M. (2019). The Relationship Between Extratropical Cyclone Strength and Atmospheric River Intensity and Position. Geophysical Research Letters, 46(3):1814-1823.

Zhu, Y. and Newell, R. E. (1994). Atmospheric rivers and bombs. Geophysical Research Letters, 21(18):1999-2002.

Zhu, Y. and Newell, R. E. (1998). A Proposed Algorithm for Moisture Fluxes from Atmospheric Rivers. Monthly Weather Review, 126:11. 


\section{Chapter 6}

\section{Appendices}

\subsection{Appendix A}

Atmospheric rivers provide a useful tool in the study of mid-latitude weather systems, due to the connection between low-level water vapour flux and impacts. In developing an AR detection algorithm for New Zealand, we took a critical look at the established methods for identifying ARs. A number of questions emerged, among them, whether IVT is the best variable for AR detection, and whether IVT intensity translates well to observed impacts.

One of the recent themes in AR science has been to accurately quantify the intensity of ARs. The scale should translate well to observed impacts, which in most cases is precipitation. Ideally, the methods to detect and quantify the intensity of ARs should involve the same variable. For example, Ralph et al. (2019) introduced a category- 5 scale, based on the intensity and duration of enhanced IVT. While developing our AR detection algorithm, we trialled a new detection variable (in an attempt to find a variable that better represents observed precipitation). We keep in mind that any detection variable should be consistent with the notion of ARs as regions of enhanced moisture flux, and be easily calculable from commonly used reanalysis fields. While investigating the impacts of ARs on precipitation, we found that IVT (and therefore ARs) may underestimate winter rainfall (when the atmosphere is closer to saturation). For this reason we trial a variable calculated by normalizing IVT by the maximum possible column water vapour (based on a vertical temperature profile). This normalization variable $\left(\mathrm{IWV}_{\max }\right)$ can be calculated as follows:

Total Column Water Vapour in $\mathrm{kg} \mathrm{m}^{-2}$ is given by:

$$
\mathrm{IWV}=\frac{1}{g} \int_{0}^{1} q \frac{\partial p}{\partial \eta} d \eta
$$


where $q$ is the specific humidity in $\mathrm{kg} \mathrm{kg}^{-1}$.

Assuming the atmospheric column is fully saturated, for a given temperature profile, the Maximum Column Water Vapour is given by:

$$
\mathrm{IWV}_{\max }=\frac{1}{g} \int_{0}^{1} q_{\max } \frac{\partial p}{\partial \eta} d \eta
$$

where $q_{\max }$ is the saturation specific humidity.

We can approximate the saturation specific humidity as

$$
q_{\text {max }} \approx 0.622 \frac{e_{\text {sat }}}{p}
$$

where we use the well known Tetens equation to approximate saturation vapour pressure:

$$
e_{\text {sat }} \approx 0.611 \exp \left(\frac{17.27 T}{T+237.3}\right)
$$

Note that normalizing IWV by the same variable would equate to the 'fraction of maximum saturation' of the atmospheric column. While the resulting variable was able to identify AR structures in reanalysis data, investigations of its ability to represent precipitation were inconclusive. As IVT is now well established as the preferred AR detection variable, we did not pursue this idea beyond a qualitative survey.

In the study of ARs, it is important to keep in mind the properties of IVT. This includes the fact that IVT consists of both dynamic (horizontal wind) and thermodynamic (moisture) components. Gonzales et al. (2020) even goes so far as to identify different 'flavours' of ARs, based on whether AR IVT is moisturedominant or wind-dominant. Although IVT has some weaknesses when considering the thermodynamic drivers of precipitation, it has the advantage of being conceptually straightforward (i.e. the number of kilograms of water vapour moving across 1 metre in 1 second). We are not aware of any studies that have used variables other than IWV or IVT as a proxy for AR detection. Although we did not pursue these ideas further, we encourage scrutiny of IVT as the preferred AR detection variable.

\subsection{Appendix B}

In this appendix we provide AR count anomalies associated with each of the climate oscillation investigated in chapter 4 . We calculate monthly anomalies of AR count by region, including counts of 'heavy' (daily precip. above 99th percentile) and 'extreme' (daily precip. above $100 \mathrm{~mm}$ ) ARs. We also calculate monthly anomalies of the number of each Kidson type concurrent with each AR, to investigate changes in the circulation patterns driving ARs. 


\begin{tabular}{|c|c|c|c|c|c|c|c|}
\hline & NZ & NNI & ENI & SWNI & NSI & ESI & WSI \\
\hline DJF & -1.31 & -0.93 & -0.99 & -0.97 & -0.84 & -0.37 & -0.44 \\
\hline MAM & 0.09 & -0.13 & 0.2 & 0.26 & 0.07 & 0.1 & -0.09 \\
\hline JJA & 0.27 & -0.59 & 0.06 & 0.13 & 0.52 & 0.55 & 1.04 \\
\hline SON & 0.11 & -0.32 & -0.43 & -0.19 & 0.51 & 0.5 & 0.82 \\
\hline
\end{tabular}

Table 6.1: AR count anomalies for positive SAM months. Bold values denote anomalies significant at the $85 \%$ level, underlined values denote anomalies significant at the $95 \%$ level.

\begin{tabular}{|c|c|c|c|c|c|c|c|}
\hline & NZ & NNI & ENI & SWNI & NSI & ESI & WSI \\
\hline DJF & -0.76 & -0.63 & -0.32 & -0.42 & -0.03 & -0.78 & -0.35 \\
\hline MAM & -0.05 & 0.21 & 0.25 & 0.29 & 0.36 & -0.01 & 0.09 \\
\hline JJA & -0.32 & -0.16 & -0.79 & -0.1 & 0.33 & 0.11 & 0.29 \\
\hline SON & 0.12 & -0.15 & -0.72 & -0.36 & -0.24 & -0.24 & 0.7 \\
\hline
\end{tabular}

Table 6.2: 'Heavy' AR count anomalies for positive SAM months. Bold values denote anomalies significant at the $85 \%$ level, underlined values denote anomalies significant at the $95 \%$ level.

\begin{tabular}{|c|c|c|c|c|c|c|c|}
\hline & NZ & NNI & ENI & SWNI & NSI & ESI & WSI \\
\hline DJF & -0.34 & -0.01 & $\underline{-0.07}$ & -0.07 & -0.07 & -0.27 & -0.26 \\
\hline MAM & 0.01 & 0.03 & 0.12 & 0.06 & 0.09 & -0.16 & -0.18 \\
\hline JJA & -0.03 & -0.24 & 0.11 & 0.21 & 0 & 0.02 & 0.2 \\
\hline SON & 0.34 & -0.17 & -0.05 & -0.08 & 0.11 & -0.16 & 0.47 \\
\hline
\end{tabular}

Table 6.3: 'Extreme' AR count anomalies for positive SAM months. Bold values denote anomalies significant at the $85 \%$ level, underlined values denote anomalies significant at the $95 \%$ level.

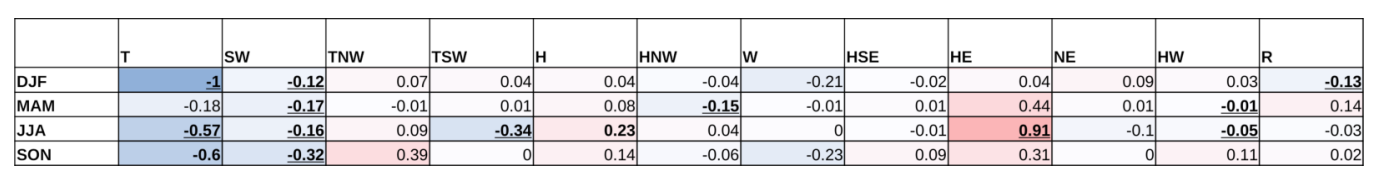

Table 6.4: AR count anomalies for each Kidson type for positive SAM months. Bold values denote anomalies significant at the $85 \%$ level, underlined values denote anomalies significant at the $95 \%$ level. 


\begin{tabular}{|c|c|c|c|c|c|c|c|}
\hline & $\mathrm{NZ}$ & NNI & ENI & SWNI & NSI & ESI & WSI \\
\hline DJF & 1.18 & 0.51 & 0.81 & 0.66 & 0.78 & 0.86 & 0.73 \\
\hline MAM & -1.1 & -0.3 & -0.6 & -0.43 & -0.5 & -0.58 & -0.64 \\
\hline JJA & -0.26 & 0.85 & 0.33 & 0.07 & -0.8 & -1.07 & -1.49 \\
\hline SON & 0.93 & 0.06 & 0.46 & 0.47 & 0.28 & 0.55 & 0.75 \\
\hline
\end{tabular}

Table 6.5: AR count anomalies for negative SAM months. Bold values denote anomalies significant at the $85 \%$ level, underlined values denote anomalies significant at the $95 \%$ level.

\begin{tabular}{|c|c|c|c|c|c|c|c|}
\hline & $\mathrm{NZ}$ & NNI & ENI & SWNI & NSI & ESI & WSI \\
\hline DJF & 1.1 & 0.28 & 0.45 & 0.5 & 0.13 & 0.86 & 0.84 \\
\hline MAM & -0.98 & -0.68 & -0.22 & -0.14 & -0.54 & -0.77 & -0.54 \\
\hline JJA & -0.32 & 0.5 & 0.45 & 0.34 & -0.65 & -1.12 & -1.15 \\
\hline SON & 0.05 & -0.49 & 0.35 & 0.16 & -0.03 & 0.47 & 0.32 \\
\hline
\end{tabular}

Table 6.6: 'Heavy' AR count anomalies for negative SAM months. Bold values denote anomalies significant at the $85 \%$ level, underlined values denote anomalies significant at the $95 \%$ level.

\begin{tabular}{|c|c|c|c|c|c|c|c|}
\hline & $\mathrm{NZ}$ & NNI & ENI & SWNI & NSI & ESI & WSI \\
\hline DJF & 0.28 & -0.11 & $\underline{-0.08}$ & -0.07 & -0.06 & 0.37 & 0.54 \\
\hline MAM & $\underline{-0.64}$ & -0.09 & 0.01 & -0.08 & $\underline{-0.2}$ & -0.03 & -0.28 \\
\hline JJA & -0.01 & 0.54 & 0.04 & -0.14 & 0.08 & -0.29 & -0.54 \\
\hline SON & -0.28 & -0.05 & -0.01 & -0.08 & 0.02 & 0.21 & -0.09 \\
\hline
\end{tabular}

Table 6.7: 'Extreme' AR count anomalies for negative SAM months. Bold values denote anomalies significant at the $85 \%$ level, underlined values denote anomalies significant at the 95\% level.

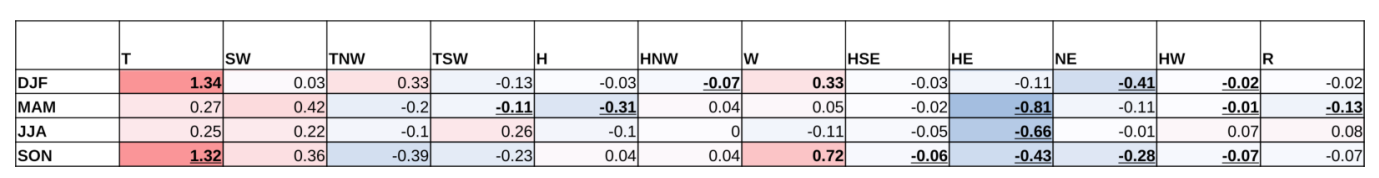

Table 6.8: AR count anomalies for each Kidson type for negative SAM months. Bold values denote anomalies significant at the $85 \%$ level, underlined values denote anomalies significant at the 95\% level. 


\begin{tabular}{|c|c|c|c|c|c|c|c|}
\hline & NZ & NNI & ENI & SWNI & NSI & ESI & WSI \\
\hline DJF & 0.15 & -0.73 & -0.45 & -0.3 & -0.26 & -0.03 & 0.52 \\
\hline MAM & 0.38 & -0.23 & -0.14 & -0.06 & 0.17 & 0.36 & 0.53 \\
\hline JJA & 0.25 & 0.01 & 0.07 & -0.2 & -0.04 & -0.03 & -0.15 \\
\hline SON & 0.68 & 0.1 & 0.04 & -0.05 & 0.36 & 0.29 & 0.64 \\
\hline
\end{tabular}

Table 6.9: AR count anomalies for El Niño months. Bold values denote anomalies significant at the $85 \%$ level, underlined values denote anomalies significant at the $95 \%$ level.

\begin{tabular}{|c|c|c|c|c|c|c|c|}
\hline & NZ & NNI & ENI & SWNI & NSI & ESI & WSI \\
\hline DJF & -0.16 & -0.06 & -0.23 & $\underline{-0.12}$ & -0.53 & -0.6 & 0.74 \\
\hline MAM & 0.33 & -0.21 & -0.12 & 0.06 & 0.02 & 0.23 & 0.41 \\
\hline JJA & 0.12 & -0.18 & -0.14 & -0.11 & -0.41 & -0.3 & -0.47 \\
\hline SON & -0.09 & -0.56 & -0.04 & 0.32 & -0.39 & -0.48 & -0.29 \\
\hline
\end{tabular}

Table 6.10: 'Heavy' AR count anomalies for El Niño months. Bold values denote anomalies significant at the $85 \%$ level, underlined values denote anomalies significant at the $95 \%$ level.

\begin{tabular}{|c|c|c|c|c|c|c|c|}
\hline & NZ & NNI & ENI & SWNI & NSI & ESI & WSI \\
\hline DJF & -0.33 & -0.33 & -0.08 & -0.18 & -0.27 & 0.31 & 0.3 \\
\hline MAM & 0.15 & -0.05 & -0.01 & 0.02 & -0.1 & 0.05 & 0.19 \\
\hline $\mathrm{JJA}$ & 0.29 & 0.06 & 0.07 & 0.04 & -0.04 & -0.21 & 0.17 \\
\hline SON & -0.26 & 0.01 & -0.01 & -0.13 & 0.18 & 0.09 & 0.02 \\
\hline
\end{tabular}

Table 6.11: 'Extreme' AR count anomalies for El Niño months. Bold values denote anomalies significant at the $85 \%$ level, underlined values denote anomalies significant at the $95 \%$ level.

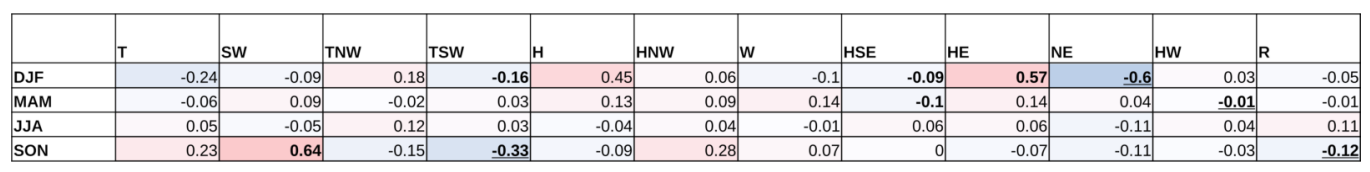

Table 6.12: AR count anomalies for each Kidson type for El Niño months. Bold values denote anomalies significant at the $85 \%$ level, underlined values denote anomalies significant at the $95 \%$ level. 


\begin{tabular}{|c|c|c|c|c|c|c|c|}
\hline & NZ & NNI & ENI & SWNI & NSI & ESI & WSI \\
\hline DJF & -0.44 & 0.09 & -0.04 & 0.09 & -0.04 & -0.15 & -0.52 \\
\hline MAM & -0.33 & 0.39 & -0.16 & -0.35 & -0.95 & -0.39 & -0.26 \\
\hline JJA & 0.02 & -0.13 & 0.51 & 0.34 & -0.21 & 0.38 & 0.33 \\
\hline SON & 0.37 & 0 & 0.18 & 0.32 & 0.26 & 0.6 & 0.53 \\
\hline
\end{tabular}

Table 6.13: AR count anomalies for La Niña months. Bold values denote anomalies significant at the $85 \%$ level, underlined values denote anomalies significant at the $95 \%$ level.

\begin{tabular}{|c|c|c|c|c|c|c|c|}
\hline & NZ & NNI & ENI & SWNI & NSI & ESI & WSI \\
\hline DJF & -0.29 & 0 & -0.07 & 0.07 & 0.31 & 0.15 & -0.49 \\
\hline MAM & -0.8 & 0.02 & -0.02 & -0.93 & -0.88 & -1.14 & -1.11 \\
\hline JJA & 0.49 & 0.55 & 0.62 & 0.9 & 0.52 & 0.49 & 1.18 \\
\hline SON & 0.61 & 0.34 & 0.65 & 0.58 & 0.37 & 0.41 & 0.56 \\
\hline
\end{tabular}

Table 6.14: 'Heavy' AR count anomalies for La Niña months. Bold values denote anomalies significant at the $85 \%$ level, underlined values denote anomalies significant at the $95 \%$ level.

\begin{tabular}{|c|c|c|c|c|c|c|c|}
\hline & NZ & NNI & ENI & SWNI & NSI & ESI & WSI \\
\hline DJF & -0.18 & 0.12 & -0.05 & -0.1 & 0.23 & -0.14 & -0.28 \\
\hline MAM & -0.34 & 0.14 & -0.13 & -0.21 & -0.08 & 0.4 & -0.07 \\
\hline JJA & -0.47 & -0.02 & -0.08 & -0.11 & 0.01 & -0.21 & -0.15 \\
\hline SON & 0.51 & 0.06 & -0.02 & 0.04 & 0 & 0.23 & 0.36 \\
\hline
\end{tabular}

Table 6.15: 'Extreme' AR count anomalies for La Niña months. Bold values denote anomalies significant at the $85 \%$ level, underlined values denote anomalies significant at the $95 \%$ level.

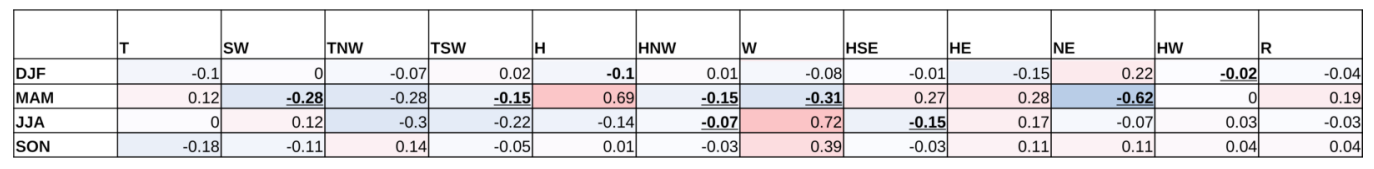

Table 6.16: AR count anomalies for each Kidson type for La Niña months. Bold values denote anomalies significant at the $85 \%$ level, underlined values denote anomalies significant at the $95 \%$ level. 


\begin{tabular}{|c|c|c|c|c|c|c|c|}
\hline & NZ & NNI & ENI & SWNI & NSI & ESI & WSI \\
\hline DJF & 1.14 & 0.2 & 0.46 & 0.4 & 0.36 & 0.43 & 0.89 \\
\hline MAM & 1.79 & -0.13 & 0.24 & 0.34 & 0.75 & $\underline{1.38}$ & $\underline{1.89}$ \\
\hline JJA & -0.51 & -0.78 & -0.47 & -0.43 & -0.32 & -0.05 & 0.13 \\
\hline SON & 0.02 & -0.24 & 0.04 & 0.01 & 0.2 & 0.19 & 0.38 \\
\hline
\end{tabular}

Table 6.17: AR count anomalies for positive IPO months. Bold values denote anomalies significant at the $85 \%$ level, underlined values denote anomalies significant at the $95 \%$ level.

\begin{tabular}{|c|c|c|c|c|c|c|c|}
\hline & NZ & NNI & ENI & SWNI & NSI & ESI & wSI \\
\hline DJF & 0.71 & 0.44 & 0.24 & 0.26 & -0.34 & 0.13 & 1.1 \\
\hline MAM & 1.1 & -0.1 & -0.2 & -0.05 & 0.53 & 1.03 & 1.58 \\
\hline JJA & -0.2 & -0.25 & -0.38 & -0.64 & 0.42 & -0.34 & -0.11 \\
\hline SON & -0.3 & -0.66 & 0.31 & 0.19 & -0.03 & 0.07 & -0.26 \\
\hline
\end{tabular}

Table 6.18: 'Heavy' AR count anomalies for positive IPO months. Bold values denote anomalies significant at the $85 \%$ level, underlined values denote anomalies significant at the $95 \%$ level.

\begin{tabular}{|c|c|c|c|c|c|c|c|}
\hline & $\mathrm{NZ}$ & NNI & ENI & SWNI & NSI & ESI & WSI \\
\hline DJF & 0.42 & -0.14 & 0.2 & 0.18 & -0.27 & 0.36 & 0.45 \\
\hline MAM & 0.87 & -0.08 & 0.09 & 0.13 & -0.08 & 0.43 & 0.83 \\
\hline JJA & 0.27 & -0.11 & 0.06 & 0.07 & 0.29 & 0.26 & -0.02 \\
\hline SON & -0.21 & 0.04 & -0.01 & -0.1 & 0.16 & -0.07 & -0.03 \\
\hline
\end{tabular}

Table 6.19: 'Extreme' AR count anomalies for positive IPO months. Bold values denote anomalies significant at the $85 \%$ level, underlined values denote anomalies significant at the $95 \%$ level.

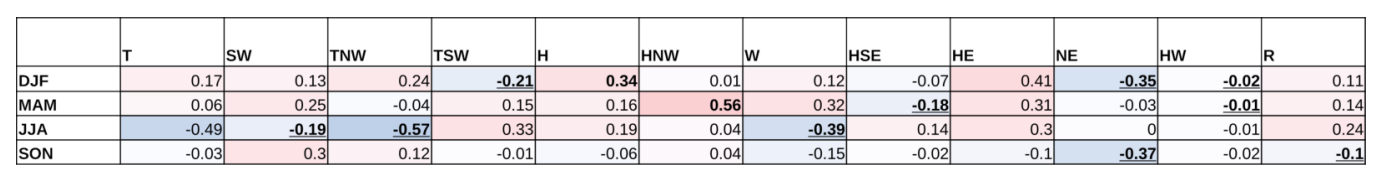

Table 6.20: AR count anomalies for each Kidson type for positive IPO months. Bold values denote anomalies significant at the $85 \%$ level, underlined values denote anomalies significant at the $95 \%$ level. 


\begin{tabular}{|c|c|c|c|c|c|c|c|}
\hline & NZ & NNI & ENI & SWNI & NSI & ESI & WSI \\
\hline DJF & -0.71 & 0.39 & 0.16 & 0.22 & -0.17 & -0.42 & -0.84 \\
\hline MAM & -0.51 & 0.68 & 0.26 & 0.22 & -0.09 & -0.1 & -0.29 \\
\hline JJA & -0.67 & -0.58 & -0.22 & 0.05 & -0.03 & 0.1 & 0.3 \\
\hline SON & -0.63 & -0.18 & 0.08 & 0.45 & 0.07 & -0.19 & -0.5 \\
\hline
\end{tabular}

Table 6.21: AR count anomalies for negative IPO months. Bold values denote anomalies significant at the $85 \%$ level, underlined values denote anomalies significant at the $95 \%$ level.

\begin{tabular}{|c|c|c|c|c|c|c|c|}
\hline & NZ & NNI & ENI & SWNI & NSI & ESI & WSI \\
\hline DJF & -0.21 & 0.3 & 0.33 & 0.73 & 0.26 & 0.12 & -0.3 \\
\hline MAM & -0.67 & -0.12 & -0.2 & -1.31 & 0.15 & -0.4 & -0.29 \\
\hline JJA & -0.52 & -0.23 & -0.79 & -0.27 & -0.19 & -0.01 & 0.99 \\
\hline SON & 0.11 & 0.92 & 0.44 & 0.4 & 0.34 & -0.13 & -0.66 \\
\hline
\end{tabular}

Table 6.22: 'Heavy' AR count anomalies for negative IPO months. Bold values denote anomalies significant at the $85 \%$ level, underlined values denote anomalies significant at the $95 \%$ level.

\begin{tabular}{|c|c|c|c|c|c|c|c|}
\hline & $\mathrm{NZ}$ & NNI & ENI & SWNI & NSI & ESI & WSI \\
\hline DJF & -0.1 & 0.11 & $\underline{-0.08}$ & -0.09 & 0.27 & -0.03 & -0.01 \\
\hline MAM & -0.02 & 0.2 & $\underline{-0.11}$ & $\underline{-0.17}$ & 0.2 & 0.22 & -0.1 \\
\hline JJA & -1.01 & -0.16 & $\underline{-0.17}$ & $\underline{-0.25}$ & -0.01 & -0.4 & -0.49 \\
\hline SON & 0.33 & 0.17 & -0.06 & 0.05 & -0.1 & 0.17 & 0.09 \\
\hline
\end{tabular}

Table 6.23: 'Extreme' AR count anomalies for negative IPO months. Bold values denote anomalies significant at the $85 \%$ level, underlined values denote anomalies significant at the 95\% level.

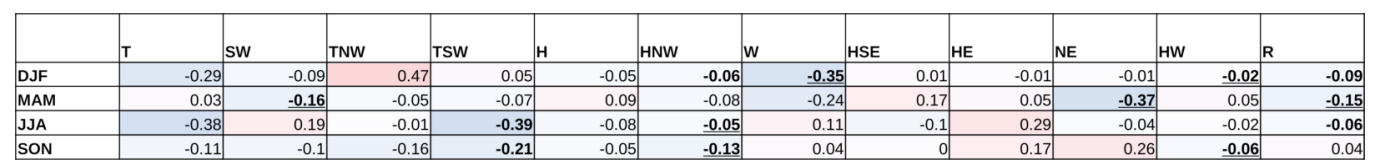

Table 6.24: AR count anomalies for each Kidson type for negative IPO months. Bold values denote anomalies significant at the $85 \%$ level, underlined values denote anomalies significant at the 95\% level. 


\begin{tabular}{|c|c|c|c|c|c|c|c|}
\hline & NZ & NNI & ENI & SWNI & NSI & ESI & WSI \\
\hline DJF & -0.44 & -0.82 & -0.55 & -0.59 & -0.31 & -0.13 & 0.28 \\
\hline MAM & -1.12 & -1.02 & -0.6 & -0.46 & -0.28 & -0.39 & -0.1 \\
\hline JJA & -0.77 & -0.93 & -0.6 & -0.55 & -0.52 & -0.4 & -0.41 \\
\hline SON & 0.31 & -0.42 & -0.26 & -0.25 & 0.55 & 0.54 & 0.74 \\
\hline
\end{tabular}

Table 6.25: AR count anomalies for positive IOD months. Bold values denote anomalies significant at the $85 \%$ level, underlined values denote anomalies significant at the $95 \%$ level.

\begin{tabular}{|c|c|c|c|c|c|c|c|}
\hline & NZ & NNI & ENI & SWNI & NSI & ESI & WSI \\
\hline DJF & -0.63 & -0.52 & -0.64 & -0.9 & -0.87 & -0.49 & 0.01 \\
\hline MAM & -1.22 & -0.94 & -0.91 & -0.89 & -0.21 & -0.31 & -0.57 \\
\hline JJA & -0.88 & -0.12 & -0.51 & -0.63 & -0.65 & -0.4 & -0.42 \\
\hline SON & 0.43 & -0.74 & -0.47 & -0.26 & 0.25 & 0.61 & 0.86 \\
\hline
\end{tabular}

Table 6.26: 'Heavy' AR count anomalies for positive IOD months. Bold values denote anomalies significant at the $85 \%$ level, underlined values denote anomalies significant at the $95 \%$ level.

\begin{tabular}{|c|c|c|c|c|c|c|c|}
\hline & $\mathrm{NZ}$ & $\mathrm{NNI}$ & ENI & SWNI & NSI & ESI & WSI \\
\hline DJF & -0.36 & $\underline{-0.22}$ & 0 & -0.08 & -0.27 & -0.02 & 0.01 \\
\hline MAM & -0.29 & -0.04 & -0.08 & -0.13 & 0.03 & 0.11 & -0.06 \\
\hline JJA & -0.16 & -0.05 & 0.12 & 0.24 & 0.04 & -0.13 & -0.23 \\
\hline SON & 0.36 & -0.06 & -0.02 & -0.04 & 0.13 & 0.64 & 0.65 \\
\hline
\end{tabular}

Table 6.27: 'Extreme' AR count anomalies for positive IOD months. Bold values denote anomalies significant at the $85 \%$ level, underlined values denote anomalies significant at the $95 \%$ level.

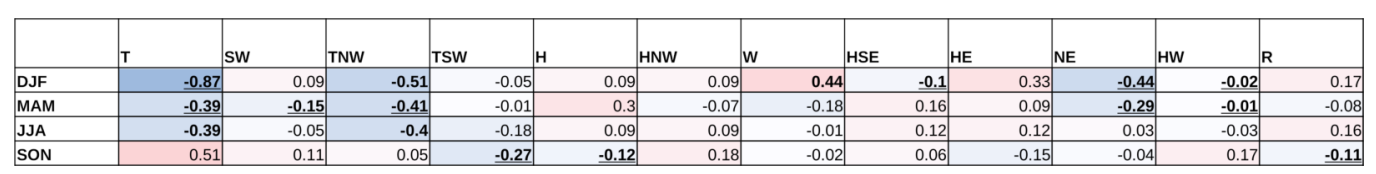

Table 6.28: AR count anomalies for each Kidson type for positive IOD months. Bold values denote anomalies significant at the $85 \%$ level, underlined values denote anomalies significant at the $95 \%$ level. 


\begin{tabular}{|c|c|c|c|c|c|c|c|}
\hline & NZ & NNI & ENI & SWNI & NSI & ESI & WSI \\
\hline DJF & -0.57 & -0.29 & \begin{tabular}{|c|}
-0.53 \\
\end{tabular} & -0.26 & \begin{tabular}{|c|} 
\\
\end{tabular} & -0.36 & -0.29 \\
\hline MAM & 0.3 & 0.45 & 0.07 & 0.09 & -0.11 & -0.2 & -0.11 \\
\hline JJA & 0.17 & 0.25 & -0.03 & 0.15 & 0.1 & 0.14 & 0.18 \\
\hline SON & 0.2 & 0.28 & 0.18 & 0.15 & 0.1 & 0.28 & -0.1 \\
\hline
\end{tabular}

Table 6.29: AR count anomalies for negative IOD months. Bold values denote anomalies significant at the $85 \%$ level, underlined values denote anomalies significant at the $95 \%$ level.

\begin{tabular}{|c|c|c|c|c|c|c|c|}
\hline & NZ & NNI & ENI & SWNI & NSI & ESI & WSI \\
\hline DJF & -0.72 & -0.12 & -0.39 & -0.03 & -0.27 & -0.4 & -0.83 \\
\hline MAM & -0.01 & -0.28 & -0.23 & -0.12 & -0.01 & -0.28 & -0.14 \\
\hline $\mathrm{JJA}$ & 0.32 & -0.26 & 0.45 & 0.24 & 0.35 & 0.12 & 0.01 \\
\hline SON & 0.54 & 0.49 & 0.59 & 0.7 & 0.29 & 0.15 & 0.15 \\
\hline
\end{tabular}

Table 6.30: 'Heavy' AR count anomalies for negative IOD months. Bold values denote anomalies significant at the $85 \%$ level, underlined values denote anomalies significant at the $95 \%$ level.

\begin{tabular}{|c|c|c|c|c|c|c|c|}
\hline & NZ & NNI & ENI & SWNI & NSI & ESI & WSI \\
\hline DJF & -0.61 & -0.09 & -0.07 & -0.1 & 0.01 & -0.36 & -0.33 \\
\hline MAM & -0.23 & 0.11 & -0.05 & -0.14 & -0.13 & -0.29 & -0.12 \\
\hline JJA & -0.34 & 0.08 & -0.01 & -0.17 & -0.15 & -0.05 & -0.35 \\
\hline SON & 0.32 & -0.08 & 0.04 & 0.05 & -0.02 & 0 & 0.14 \\
\hline
\end{tabular}

Table 6.31: 'Extreme' AR count anomalies for negative IOD months. Bold values denote anomalies significant at the $85 \%$ level, underlined values denote anomalies significant at the 95\% level.

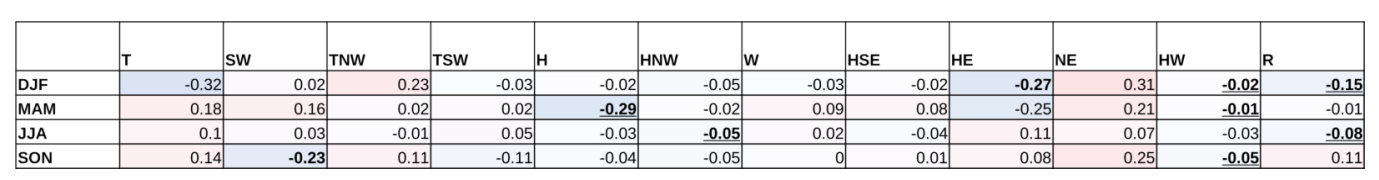

Table 6.32: AR count anomalies for each Kidson type for negative IOD months. Bold values denote anomalies significant at the $85 \%$ level, underlined values denote anomalies significant at the 95\% level. 


\subsection{Appendix C}

\subsubsection{AR Case Study: March 2019}

\section{Model Configuration}

The Weather Research and Forecasting (WRF) Model Version 4.0 (Skamarock et al., 2019) is used to simulate case study AR events. The WRF Model is a nextgeneration numerical weather prediction system used for atmospheric research and operational forecasting. Initial and boundary conditions are forced by 6hourly 0.25 degree ( $27 \mathrm{~km}$ ) GFS analysis. To transition from the relatively coarse GFS forcing data to a high resolution simulation, we implement a nested domain configuration. A large outer domain at $9 \mathrm{~km}$ resolution extends over much of the south-west pacific. This provides high-resolution coverage over the complete spatial extent of the ARs. A $3 \mathrm{~km}$ resolution inner domain over New Zealand provides detailed data at landfall. Similar domain configurations are implemented operationally, such as the WRF based limited-area model operated by Metservice. Figure 6.1 shows the domain setup. The inner and outer domains are run as a two-way nest, meaning the values of the coarse domain are overwritten by the output of the nested domain.

We implement the New Thompson microphysics scheme (Thompson et al., 2008), the RRTMG shortwave and longwave radiation schemes (Iacono et al., 2008), Mellor-Yamada Nakanishi and Niino Level 2.5 planetary boundary layer scheme (Nakanishi \& Niino, 2006), and Noah land surface Model (Niu et al., 2011). The Kain-Fritsch cumulus scheme (Kain, 2004) is applied for the $9 \mathrm{~km}$ outer domain only. The model setup uses 44 vertical levels extending to $50 \mathrm{hPa}$, with a larger fraction of levels nearer the surface as we are primarily interested in low-level processes. The integration time step used is 45 seconds for the outer domain and 15 seconds for the inner domain. Model data is output every hour over the event duration for both domains. A summary of the model settings is provided in Table 6.33 .

\subsubsection{Event Simulation}

In general, IVT values exceeding $750 \mathrm{kgm}^{-1} \mathrm{~s}^{-1}$ are considered notable. Values over $1000 \mathrm{kgm}^{-1} \mathrm{~s}^{-1}$ are considered extreme, and often indicate a significant flooding threat. The prolonged period of moisture flux and orographic forcing, was responsible for driving event rainfall totals of over a meter for parts of the Southern Alps. The AR cross section is shown in figure 6.2. The vertical structure shows a low level jet ahead of the cold front, driving a strong inner AR core and strong northwest flow aloft. Over that cross section the flow rate of moisture exceeded the equivalent liquid water flow rate through the mouth of the Amazon 


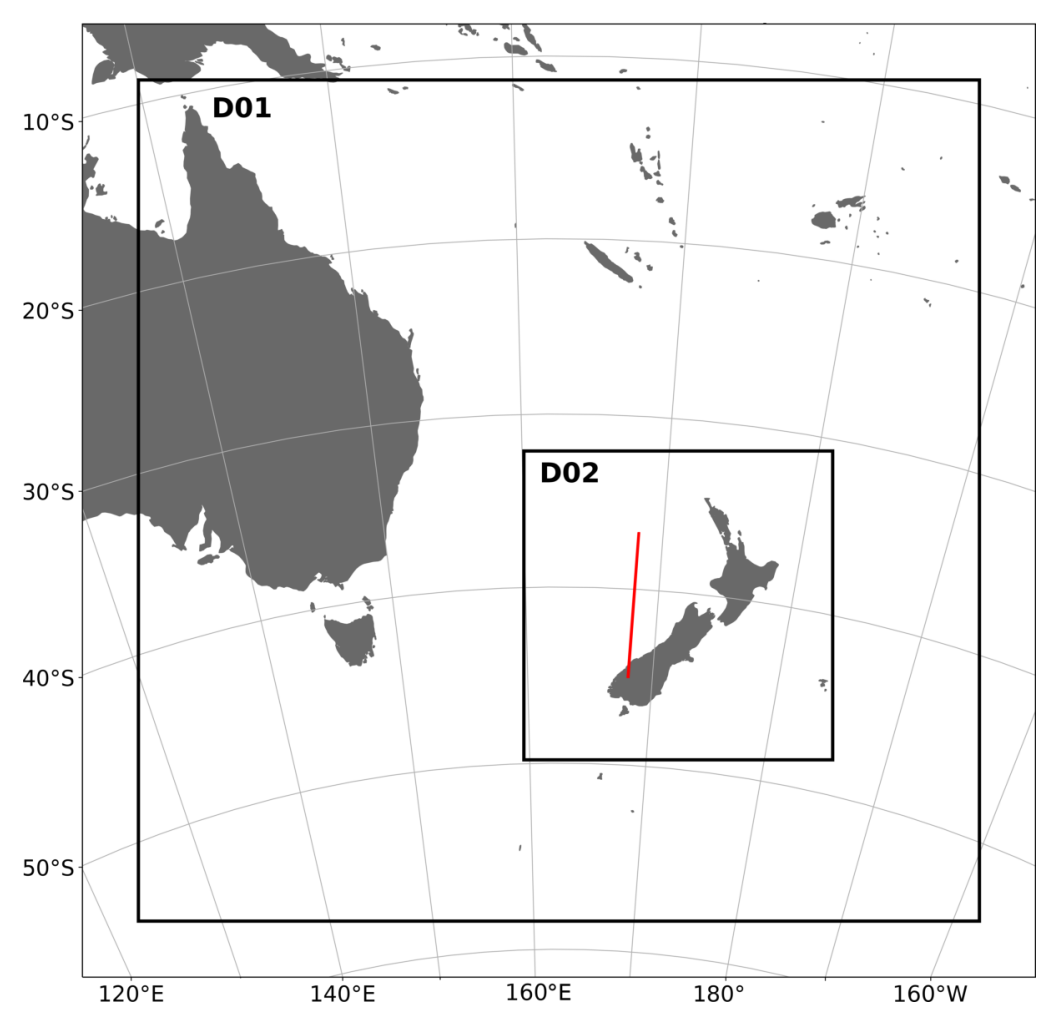

Figure 6.1: WRF nested domain configuration. The red line depicts the cross-section used in Figure 6.2

river. 


\begin{tabular}{cc}
\hline Parameter & Setting \\
\hline Initialisation and forcing & GFS analysis (6 hrly, 0.25 degree) \\
Grid spacing & $9 \mathrm{~km}, 3 \mathrm{~km}$ \\
Vertical levels & 44 \\
Time step & $45 \mathrm{~s}$ \\
Nesting & Two-way \\
Microphysics & New Thompson \\
Longwave radiation & RRTMG \\
Shortwave radiation & RRTMG \\
Surface physics & Noah Land Surface Model \\
Planetary Boundary Layer physics & Mellor-Yamada Nakanishi Niino 2.5 \\
Cumulus physics & Kain-Fritsch \\
\hline
\end{tabular}

Table 6.33: WRF namelist settings for each simulation. 
(a)

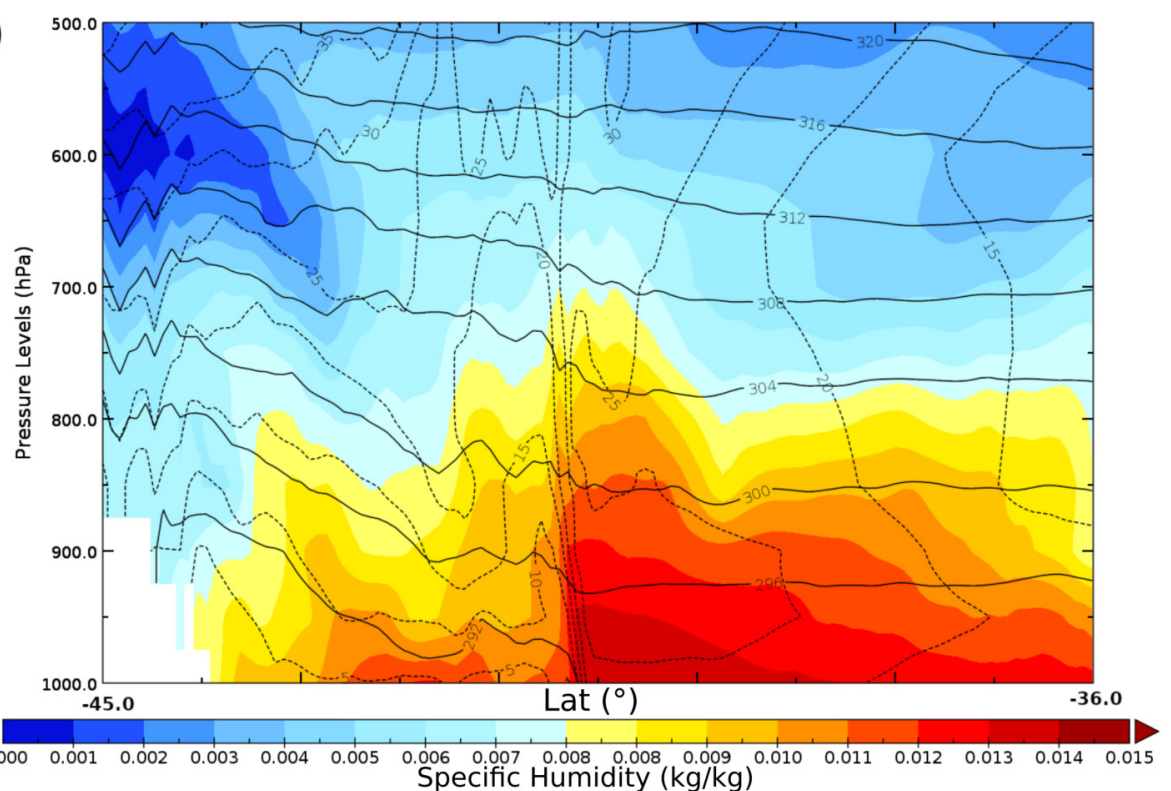

(b)

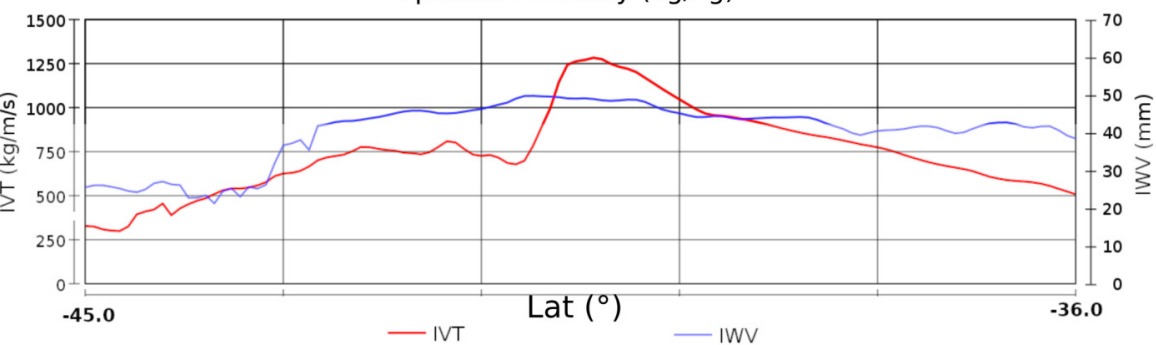

Figure 6.2: Atmospheric river cross section (2019-03-26-03 UTC) showing (a) specific humidity (coloured contours), potential temperature (black contours), and wind speed (dashed contours). (b) IVT and IWV values over the cross section. 